\title{
Delivering anion transporters to lipid bilayers in water
}

Daniel A. McNaughton, ${ }^{a}$ Tsz Ying (Teresa) To, ${ }^{a, b}$ Bryson A. Hawkins, ${ }^{c}$ David E. Hibbs, ${ }^{c}$ and Philip A. Gale ${ }^{a, d^{*}}$

Cyclodextrins have been employed as delivery agents of lipophilic anion transporters, which allow their incorporation into lipid bilayers without using an organic solvent or pre-incorporation.

The development of synthetic anion transporter systems to facilitate the transport of chloride or $\mathrm{HCl}$ across lipid bilayers ${ }^{1}$ has been driven in recent years by the potential to discover new anti-cancer agents' ${ }^{2}$ and the development of 'channel replacement therapies' 3 to ameliorate the symptoms of diseases such as cystic fibrosis. Current approaches to delivering these anionophores to model lipid bilayer systems include premixing the lipids with the transporter before vesicle formation (pre-incorporation) $^{4}$ or delivering the transporter to a suspension of vesicles in a small quantity of DMSO or other organic solvents. ${ }^{5}$ Neither delivery method is ideal when studying the effect of anionophores on biological systems beyond cell cultures.

Cyclodextrins (CD) are a commercially available class of cyclic oligosaccharide ${ }^{6}$ that have been used to encapsulate drug molecules to improve their water solubility and bioavailability. ${ }^{7}$ We decided to employ this methodology to improve the deliverability of anionophores to lipid bilayer membranes. Adamantyl moieties form stable inclusion complexes with $\beta-C D$ (typically $K_{\mathrm{a}}=10^{3}-10^{5} \mathrm{M}^{-1}$ ) due to their perfect size-fit complementarity with the hydrophobic cavity of $\beta-C D .8$ Therefore, a novel series of adamantyl-appended transporters 1-6 (Fig. 1) were prepared with the intention that the formation of an inclusion complex with a $\beta-C D$ derivative would enhance the aqueous solubility of the transporters, permitting their delivery to liposomal assays in pure water. Successful aqueous delivery would allow these types of compounds to be studied in biological systems while avoiding potential toxicity from the use of an organic solvent.

\footnotetext{
a. School of Chemistry (F11), The University of Sydney, NSW 2006, Australia. philip.gale@sydney.edu.au

b. Chemistry, University of Southampton, Southampton, SO17 1BJ, UK.

c. School of Pharmacy, The University of Sydney, NSW 2006, Australia.

d. The University of Sydney Nano Institute (Sydney Nano), The University of Sydney, NSW 2006, Australia.
}

Novel mono-(thio)urea compounds $\mathbf{1}, \mathbf{2}, \mathbf{3}$, and 4 were prepared via condensation reactions between commercially available 1-adamantanemethylamine or 1-adamantylamine and the corresponding iso(thio)cyanate. Compounds $\mathbf{3}$ and $\mathbf{6}$ were readily obtained by reaction of either aminoadamantyl derivative with a 4-nitrophenyl squarate intermediate (see the ESIt for the experimental details, synthetic methods, and characterisation data). Single crystals of compound $\mathbf{1}$ suitable for X-ray diffraction were obtained in the presence of tetrabutylammonium chloride (TBACl) (20 equiv.) by slow evaporation of a saturated DMSO solution containing the two components. 1.TBACl crystallised in the centrosymmetric triclinic $\mathrm{P} \overline{1}$ space group (CDCC 2109213). The receptor was found to form a 1:1 receptor:anion complex with $\mathrm{TBACl}$, shown in Fig 2, with coordinating hydrogen bonding interactions existing between chloride and the protons of the urea moiety $(\mathrm{N} \cdots \mathrm{Cl}$ distances: $3.145-3.278 \AA, \quad \mathrm{N}-\mathrm{H} \cdots \mathrm{Cl}$ angles: $\left.150.5(7)-165.6(7)^{\circ}\right)$. The crystal structures of compounds 1 and $\mathbf{3}$ are available in the ESIt.

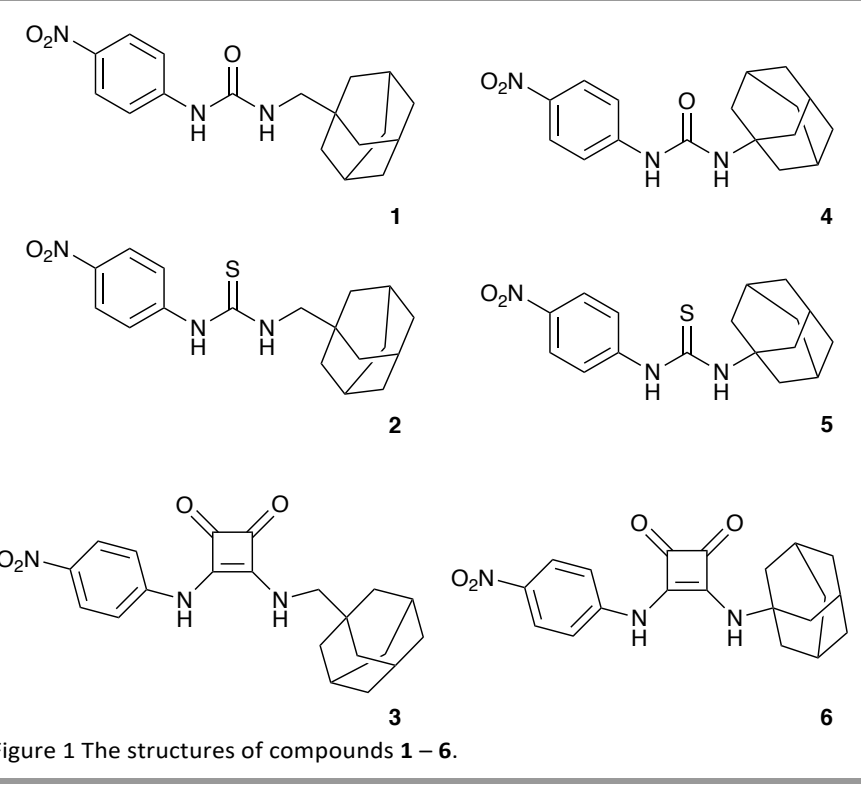

Proton NMR titrations were conducted for compounds 1-6 to assess their affinity for binding $\mathrm{Cl}^{-}$(as TBACl) in DMSO- $d_{6} / 0.5 \%$ 
$\mathrm{D}_{2} \mathrm{O}$. The changes in the chemical shifts of the $\mathrm{NH}$ protons in each titration were followed and fit to a 1:1 host:guest binding model using the Bindfit applet. ${ }^{9}$

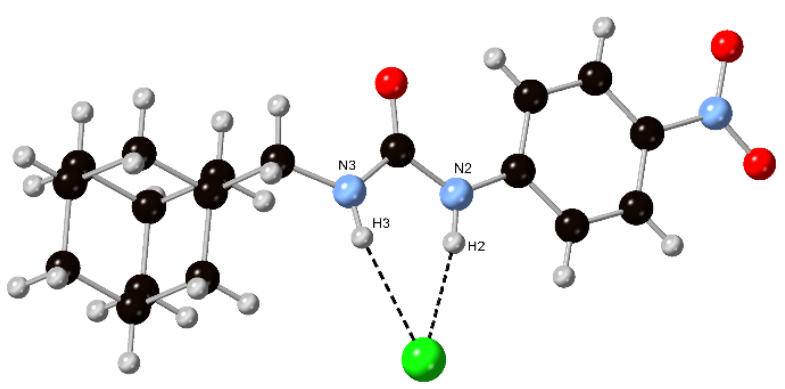

Figure 1 The single crystal X-ray structure of 1.TBACl complex (CDCC 2109213). The tetrabutylammonium counter cation has been omitted for clarity.

As shown in Table 1, compounds 1-6 exhibit weak to moderate 1:1 binding to $\mathrm{Cl}^{-}$in the competitive solvent media employed. The binding constants range from 21-249 $\mathrm{M}^{-1}$ and correlate with the acidity and potency of each species of hydrogen bond donor, and squaramide compounds $\mathbf{3}$ and $\mathbf{6}$ exhibits the series' highest binding affinity. Compounds $\mathbf{1 - 3}$, which possess a methyl spacer incorporated between the hydrogen bond donor moiety and the adamantyl group, are stronger $\mathrm{Cl}^{-}$binding receptors than compounds 4-6. This difference in binding affinity suggests that the proximity of the adamantyl groups to the binding site could interfere with the approach of $\mathrm{Cl}^{-}$during coordination and that binding is more efficient when the adamantyl group is permitted to orientate away from the binding site.

Table 1. Binding constants $\left(K_{\mathrm{a}}\right)$ for the complexation of compounds 1-6 and $\mathrm{Cl}^{-}$(as the TBA salt) in DMSO- $d_{6} / 0.5 \% \mathrm{D}_{2} \mathrm{O}$ at $298 \mathrm{~K}$

\begin{tabular}{cccc}
\hline Compound & $K_{\mathrm{a}}\left(\mathrm{M}^{-1}\right)^{\mathrm{a}, \mathrm{b}}$ & Compound & $K_{\mathrm{a}}\left(\mathrm{M}^{-1}\right)^{\mathrm{a}, \mathrm{b}}$ \\
\hline $\mathbf{1}$ & 34 & $\mathbf{4}$ & 21 \\
$\mathbf{2}$ & 48 & $\mathbf{5}$ & 39 \\
$\mathbf{3}$ & 249 & $\mathbf{6}$ & 211
\end{tabular}

a All errors $<6 \%$. ${ }^{b}$ Calculated with a 1:1 host:guest binding model using the online Bindfit applet. ${ }^{9}$

The chloride transport activity of compounds 1-6 was assessed using the $\mathrm{Cl}^{-} / \mathrm{NO}_{3}{ }^{-}$exchange ISE assay. For these experiments, receptors were delivered to the experimental solution as an aliquot of DMSO solution. Large unilamellar vesicles $(200 \mathrm{~nm}$ ) were prepared with a $\mathrm{NaCl}$ internal solution (487 mM) buffered to $\mathrm{pH} 7.2$ with sodium phosphate salts (5 $\mathrm{mM})$. The vesicles were suspended in an external solution of $\mathrm{NaNO}_{3}$ (487 mM), which was also buffered to $\mathrm{pH} 7.2$ with sodium phosphate salts $(5 \mathrm{mM})$ to give a lipid concentration of $1 \mathrm{mM}$. The experiments were initiated by adding a transporter as a DMSO aliquot ( $10 \mu \mathrm{L}$ to a total volume of $5 \mathrm{~mL})$ at $t=0 \mathrm{~s}$, and chloride efflux was followed using a chloride ISE. Doseresponse studies were performed across several concentrations of the transporter. Hill analysis was applied to obtain $\mathrm{EC}_{50}$ values (the concentration of transporter needed to achieve $50 \%$ chloride efflux after $270 \mathrm{~s}$ ), Hill coefficients (which indicate the stoichiometry of the transport process), and initial transport rate at a 2 mol\% loading of the transporter, with respect to concentration of lipid, which are shown in Table 2. In general, the trend in transporter activity reflects the trend in $\mathrm{Cl}^{-}$binding affinity. Compound $\mathbf{3}$ was less active in the assay than expected, likely due to the poor solubility of this compound in aqueous solution. The thiourea and squaramide transporters exhibit reasonable levels of transport activity $\left(\mathrm{EC}_{50}=0.31-0.47 \mathrm{~mol} \%\right)$ and are comparable to other functionalised receptors that possess only a single hydrogen bond donor. ${ }^{10}$ The Hill coefficients ranged from $n=1.6-2.7$ and indicate a 2:1 transporter:chloride stoichiometry is required to facilitate transport. A 2:1 sandwich complex offers a greater degree of encapsulation around the chloride anion, shielding the charged species whilst traversing the non-polar interior of the bilayer. The initial transport rate calculated for $\mathbf{3}$ is the slowest of the series $\left(k_{\text {ini }}=0.5 \% \mathrm{~s}^{-1}\right)$, despite the enhanced activity over the urea compounds 1 and 4 ( $k_{\text {ini }}=1.7$ and $1.0 \% \mathrm{~s}^{-1}$, respectively). This result again suggests that poor solubility impacts the rate of delivery of this transporter into the membrane.

Table 2. Summary of the anion transport parameters ( $\mathrm{EC}_{50}$ and $\mathrm{n}$ ), initial transport rates at a $2 \mathrm{~mol} \%$ loading of the transporter (kini), and calculated lipophilicity values (clog P) for compounds 1-6.

\begin{tabular}{cccc}
\hline Compound & EC $_{50}(\mathrm{~mol} \%)^{\mathrm{a}}$ & $\begin{array}{c}\text { Hill } \\
\text { coefficient }(\mathrm{n})\end{array}$ & $\begin{array}{c}\text { Initial transport } \\
\text { rate }\left(k_{\text {ini }}\right)\left(\% \mathrm{~s}^{-1}\right)^{\mathrm{b}}\end{array}$ \\
\hline $\mathbf{1}$ & 0.80 & 2.4 & 1.7 \\
$\mathbf{2}$ & 0.37 & 2.4 & 11.0 \\
$\mathbf{3}$ & 0.47 & 1.8 & $0.5^{\mathrm{c}}$ \\
$\mathbf{4}$ & 0.95 & 2.1 & 1.0 \\
$\mathbf{5}$ & 0.45 & 1.6 & 3.8 \\
$\mathbf{6}$ & 0.31 & 2.7 & 3.1
\end{tabular}

a The $\mathrm{EC}_{50}$ at $t=270 \mathrm{~s}$, shown as transporter:lipid molar percent. b Max initial transport rate $\left(\% \mathrm{~s}^{-1}\right)$ calculated at a transporter loading of $2 \mathrm{~mol} \%$ by fitting an efflux plot to an exponential decay function. ${ }^{c}$ Efflux plot fit to Boltzmann sigmoidal curve.

2-Hydroxypropyl- $\beta$-cyclodextrin (HP- $\beta$-CD), shown in Fig. 3, was employed as the host molecule due to its enhanced aqueous solubility relative to unsubstituted $\beta$-cyclodextrin. The formation of an inclusion complex with compounds 1-6 was attempted using a kneading method (method 1), and two methods involving microwave irradiation (methods 2 and 3 ), which differ in the amount of solvent used.11 Each method involved using a 1:1 water:ethanol solvent mixture to ensure that both host and guest were partially solubilised, and each inclusion complex mixture was added to pure water after the completion of the inclusion process. The addition of water resulted in the precipitation of any uncomplexed transporter, which was filtered and removed, leaving a filtrate containing a mixture of free HP- $\beta-C D$ host and the desired inclusion complex. A full description of the methods used to obtain the inclusion complexes can be found in the ESIt.

Evidence for the formation of an inclusion complex following each preparation method was initially assessed by obtaining a ${ }^{1} \mathrm{H}$ NMR spectrum of each inclusion complex mixture in $\mathrm{D}_{2} \mathrm{O}$. The presence of signals that can be reliably attributed to the guest transporter indicates that the molecule exhibits enhanced aqueous solubility due to the inclusion process. In the ${ }^{1} \mathrm{H} N M R$ spectra of HP- $\beta-C D \cdot 1$ (methods $1-3), H P-\beta-C D \cdot 2$ (methods $1-3$ ), 
HP- $\beta$-CD.4 (methods 1-3), and HP- $\beta-C D \cdot 6$ (methods 1-2), peaks attributed to the guest transporter are clearly visible and integrated to the expected ratio (shown in the ESI+).

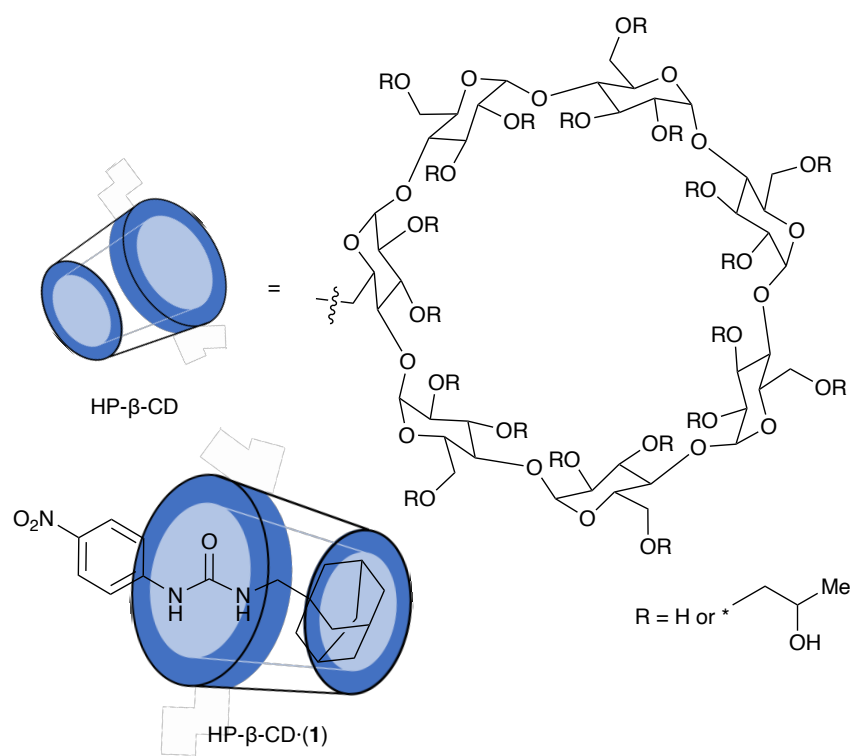

Figure 3 A schematic showing encapsulation of transporter $\mathbf{1}$ in HP- $\beta-C D$.

The inclusion complex was isolated as part of a mixture of free HP- $\beta-C D$ and inclusion complex. The mass percentage of transporter present in each mixture was determined using UVVis studies in DMSO. Initially, UV-Vis spectra were collected at five concentrations (5-50 $\mu \mathrm{M})$ for compounds 1-6, calibration curves were constructed, and the molar extinction coefficient, $\varepsilon$, for each transporter was calculated. HP- $\beta-C D$ is transparent in the tested UV-Vis region (Fig S96, ESI + ), meaning any absorbances in the UV-Vis spectra of the inclusion complex mixtures were attributed solely to the transporter molecule. A mass for each inclusion complex mixture was recorded before a UV-Vis spectrum was obtained. The absorbance value, $A$, and the calculated $\varepsilon$ in each case was used to determine the concentration of transporter present in the DMSO solutions and, therefore, the mass percentage of transporter relative to $\mathrm{HP}-\beta-C D$ in each mixture. Full details of the calculations and the mass percentages values for each inclusion mixture are reported in Section 9.2 of the ESIt.

Mass percentages of transporter present ranged from 1.8-6.9\% (Table S10). The mixtures containing the highest mass percentage corresponded to approximately a 3:1 molar ratio of HP- $\beta-C D$ to transporter. The microwave irradiation methods were generally more successful than kneading, which agrees with findings in the literature. ${ }^{12}$ The inclusion of compound $\mathbf{3}$ was unsuccessful, which is likely due to the poor solubility of this receptor. The inclusion of compound $\mathbf{5}$ was similarly insufficient. The proximity of the adamantane to the bulky thiourea sulfur atom of this compound may sterically inhibit the approach of HP- $\beta-C D$. A mass percentage of $>6 \%$ was calculated for compounds $\mathbf{6}$ (method 2 ) and 1, 2, and $\mathbf{4}$ (method 3 ), and these inclusion complex mixtures were investigated further.

Additional UV-Vis spectra were collected for HP- $\beta-C D \cdot \mathbf{1}, \mathrm{HP}-$ $\beta-C D \cdot 2, H P-\beta-C D \cdot 4$ and HP- $\beta-C D \cdot 6$, dissolved in $\mathrm{H}_{2} \mathrm{O}$, and compared to the UV-Vis spectra of the free compounds in DMSO. An overlay of the UV-Vis traces of free transporter 1 dissolved in DMSO and the HP- $\beta-C D \cdot 1$ complex dissolved in water can be viewed in Fig S97. The similarity in the absorbance profile at similar wavelengths of $342 \mathrm{~nm}\left(\mathrm{H}_{2} \mathrm{O}\right)$ and $354 \mathrm{~nm}$ (DMSO) suggested that compound $\mathbf{1}$ is present in the inclusion mixture dissolved in $\mathrm{H}_{2} \mathrm{O}$ and that an inclusion complex has been prepared successfully. Overlaid spectra of the other inclusion complexes are shown in the ESIt.

FTIR spectra of the four inclusion complex mixtures and the free compounds were obtained, and the characteristic stretches were compared. A decrease in intensity, a shift in wavenumber, or the disappearance of stretches associated with the guest can all support the formation of an inclusion complex due to restriction of the guest stretching vibrations when included within the HP- $\beta-C D$ cavity. ${ }^{13}$ However, the high mass content of HP- $\beta-C D$ present means bands associated with this component are more intense, leading to overlap and obscuring of bands associated with the guest molecules. ${ }^{14}$ Nevertheless, distinct differences were observed upon a comparison of the spectra, described in the ESIt.

The 2D NOESY ${ }^{1} \mathrm{H}$ NMR spectra collected for the four mixtures (Fig S118-S129) provided the strongest evidence for the formation of inclusion complexes. NOE cross-peaks can be clearly seen between the resonances attributed to the guest adamantyl protons and the protons which line the internal cavity of HP- $\beta-C D$. This proximity suggests that an inclusion complex has formed between the two components and is complemented by the absence of cross-peak correlations associated with other HP- $\beta-C D$ protons located on the outer surface of the structure. Interestingly, cross-peak interactions were also visible between the internal HP- $\beta-C D$ protons and the signals related to the aryl protons of the guest molecules. One explanation for these findings is that the adamantane sits in the primary rim of HP- $\beta-C D$ whilst the remainder of the molecule penetrates the cavity. Another possibility is that both end groups are included inside a CD molecule, forming a 2:1 host:guest complex. Both configurations would minimise the exposure of the aromatic unit to surrounding water molecules, which otherwise would inhibit aqueous solubility.

The transport capabilities of the inclusion complexes were investigated using the $\mathrm{Cl}^{-} / \mathrm{NO}_{3}{ }^{-}$exchange ISE assay. The conditions employed were near-identical to those employed for studies on the free transporter; however, the HP- $\beta$ $\mathrm{CD}$ transporter complexes were delivered in an aliquot of pure water. The transporter mass percentage values calculated previously were applied to provide the mass of the inclusion complex required to give a certain concentration of transporter (mol\%) in aqueous solution. A control run with only HP- $\beta-C D$ present resulted in no $\mathrm{Cl}^{-}$efflux. The transport parameters calculated for each complex are shown in Table 3.

Encouragingly, the results indicated that the transporters could be successfully delivered to the vesicles and that delivery as an inclusion complex facilitated $\mathrm{Cl}^{-}$transport with minimal depreciation in activity. The dose response efflux plots for $\mathbf{1}$ when delivered in DMSO are very similar to the efflux plots for inclusion complex HP- $\beta-C D \cdot 1$ when delivered in $\mathrm{H}_{2} \mathrm{O}$, which are 
both shown in Fig. 4. The $\mathrm{EC}_{50}$ values of the inclusion complexes all differ by less than $30 \%$ of the DMSO-delivered transporters in Table 2. Comparable levels of activity are gratifying but perhaps surprising result. To cross the membrane, the transporter molecule must first decomplex from the CD host. These findings show that this process does not hamper deliverability into the lipid bilayer.
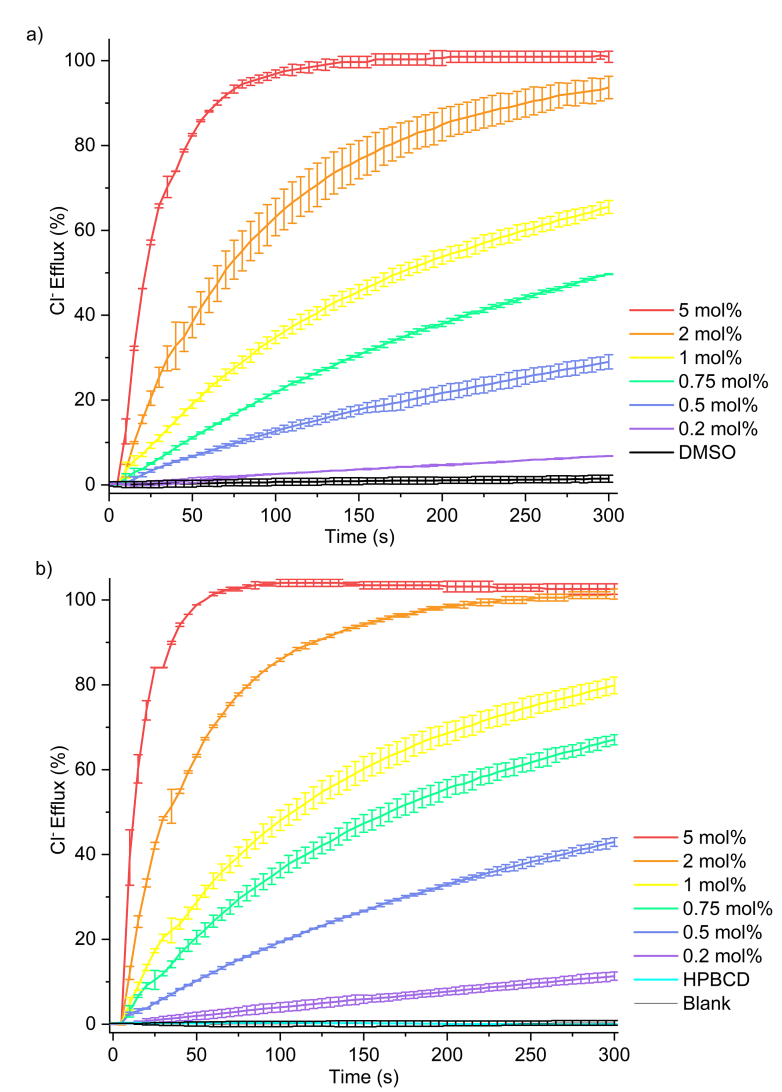

Figure 4 Dose-response studies for compound 1 using the $\mathrm{Cl}^{-} / \mathrm{NO}_{3}{ }^{-}$ISE assay. a) Delivered as a free transporter in DMSO solution. b) Delivered as an inclusion complex HP- $\beta-C D \cdot 1$ in water. Each data point is the average of two repeats with error bars to show standard deviation.

In summary, a series of adamantyl-functionalised transporters were synthesised and included inside the cavity of HP- $\beta$-CD to improve their aqueous deliverability. Following confirmation of the formation of inclusion complexes, liposomal chloride transport studies found that the complexes did not limit transport activity compared with delivery of the anionophores in DMSO. These findings highlight the potential for cyclodextrin inclusion complexes to be employed as delivery vehicles to examine the properties of anion transporters in vitro and in vivo in the absence of DMSO. Deliverability and activity in purely aqueous environments are key steps towards anion transporters finding future real-world applications.

DAM, TYT, BH, DEH and PAG acknowledge and pay respect to the Gadigal people of the Eora Nation, the traditional owners of the land on which we research, teach and collaborate at the University of Sydney. PAG thanks the Australian Research Council (DP200100453) and the University of Sydney for funding.
Table 3 Summary of the anion transport parameters $\left(E_{50}\right.$ and $\left.n\right)$ and initial transport rates at a 2 mol\% loading of the transporter $\left(k_{\text {ini }}\right)$ for the HP- $\beta$-CD-transporter inclusion complexes, delivered to vesicles as solutions of pure water

\begin{tabular}{|c|c|c|c|}
\hline Compound & $\begin{array}{c}\mathrm{EC}_{50} \\
(\mathrm{~mol} \%)^{\mathrm{a}}\end{array}$ & $\begin{array}{l}\text { Hill Coefficient } \\
\text { (n) }\end{array}$ & $\begin{array}{c}\text { Initial } \\
\text { Transport Rate } \\
\left(k_{\text {ini }}\right) \\
\left(\% \mathrm{~s}^{-1}\right)^{\mathrm{b}}\end{array}$ \\
\hline HP- $\beta-C D \cdot 1$ & 0.62 & 3.3 & 4.8 \\
\hline$H P-\beta-C D \cdot 2$ & 0.38 & 1.2 & 23.6 \\
\hline$H P-\beta-C D \cdot 4$ & 1.22 & 1.9 & 0.6 \\
\hline HP- $\beta-C D \cdot 6$ & 0.39 & 1.9 & 2.2 \\
\hline
\end{tabular}

a The $\mathrm{EC}_{50}$ at $t=270 \mathrm{~s}$, shown as transporter:lipid molar percent. ${ }^{\mathrm{b}}$ Max initial transport rate $\left(\% \mathrm{~s}^{-1}\right)$ calculated at a transporter loading of 2 mol\% by fitting an efflux plot to an exponential decay function.

\section{Conflicts of Interest}

There are no conflicts of interest to declare.

\section{Notes and references}

1 J.T. Davis, P.A. Gale and R. Quesada, Chem. Soc. Rev., 2020, 49, 6056-6086.

2 (a) S.-H. Park, S.-H. Park, E.N.W. Howe, J.Y. Hyun, L.-J. Chen, I. Huang, G. Vargas-Zuñiga, N. Busschaert, P.A. Gale, J.L. Sessler, I. Shin, Chem, 2019, 5, 2079-2098; (b) N. Busschaert, S.-H. Park, K.-H. Baek, Y.P. Choi, J. Park, E.N.W. Howe, J.R. Hiscock, L.E. Karagiannidis, I. Marques, V. Félix, W. Namkung, J.L. Sessler, P.A. Gale and I. Shin, Nature Chem., 2017, 9, 667675.

3 H. Li, H. Valkenier, A.G. Thorne, C.M. Dias, J.A. Cooper, M. Kieffer, N. Busschaert, P.A. Gale, D.N. Sheppard and A.P. Davis, Chem. Sci. 2019, 10, 9663-9672.

4 J.A. Cooper, S. T. G. Street, A. P. Davis, Angew. Chem. Int. Ed., 2014, 53, 5609-5613.

5 L.A. Jowett and P.A. Gale, Supramol. Chem., 2019, 31, 297312.

6 (a) J. Szejtli, Chem. Rev., 1998, 98, 1743-1754; (b) G. Crini, Chem. Rev., 2014, 114, 10940-10975.

7 A. Haimhoffer, Á. Rusznyák, K. Réti-Nagy, G. Vasvári, J. Váradi, M. Vecsernyés, I. Bácskay, P. Fehér, Z. Ujhelyi and F. Fenyvesi, Sci. Pharm., 2019, 87, 33.

8 R. Palepu and V. C. Reinsborough, Aust. J. Chem., 1990, 43, 2119-2123.

9 D. B. Hibbert and P. Thordarson, Chem. Commun., 2016, 52 12792-12805.

10 (a) S. N. Berry, V. Soto-Cerrato, E. N. W. Howe, H. J. Clarke, I. Mistry, A. Tavassoli, Y.-T. Chang, R. Pérez-Tomás and P. A. Gale, Chem. Sci., 2016, 7, 5069-5077; (b) X. Bao, X. Wu, S. N. Berry, E. N. W. Howe, Y.-T. Chang and P. A. Gale, Chem. Commun., 2018, 54, 1363-1366.

11 (a) K. P. Sambasevam, S. Mohamad, N. M. Sarih and N. A. Ismail, Int. J. Mol. Sci., 2013, 14, 3671-3682; (b) S. Riela, G. Lazzara, P. Lo Meo, S. Guernelli, F. D'Anna, S. Milioto and R. Noto, Supramol. Chem., 2011, 23, 819-828.

12 (a) A. Figueiras, L. Ribeiro, M. T. Vieira and F. Veiga, J. Incl. Phenom. Macrocycl. Chem., 2007, 57, 173-177; (b) S. Prabu, M. Swaminathan, K. Sivakumar and R. Rajamohan, J. Mol. Struct., 2015, 1099, 616-624.

13 P. Mura, J. Pharm. Biomed. Anal., 2015, 113, 226-238.

14 O. Adeoye, C. Costa, T. Casimiro, A. Aguiar-Ricardo and H. Cabral-Marques, J. Supercrit. Fluids, 2018, 133, 479-485. 


\section{Delivering anion transporters to lipid bilayers in water}

Daniel A. McNaughton, ${ }^{\mathrm{a}}$ Tsz Ying (Teresa) To, ${ }^{\mathrm{a}, \mathrm{b}}$ Bryson A. Hawkins, ${ }^{\mathrm{c}}$ David E. Hibbs, ${ }^{\mathrm{c}}$ and Philip A. Gale. ${ }^{\text {a,d }}{ }^{*}$

a. School of Chemistry (F11), The University of Sydney, NSW, 2006, Australia.

b. Chemistry, University of Southampton, Southampton, SO17 1BJ, UK.

c. School of Pharmacy, The University of Sydney, NSW, 2006, Australia.

d. The University of Sydney Nano Institute (Sydney Nano), The University of Sydney, NSW, 2006, Australia.

*E-mail: philip.gale@sydney.edu.au

\section{Contents}

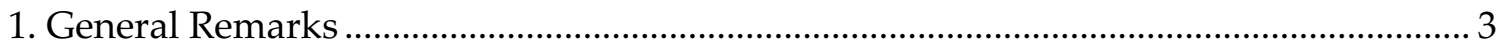

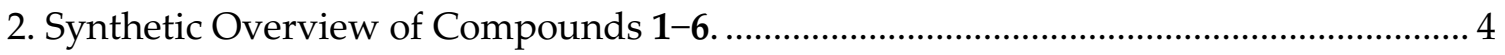

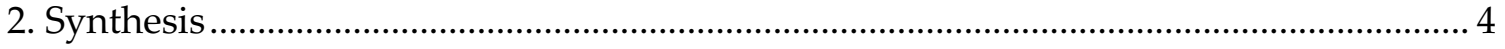

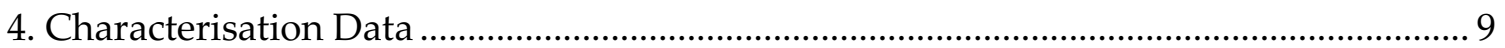

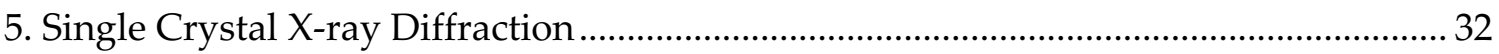

6. Proton NMR Titration Anion Binding Studies .................................................................. 43

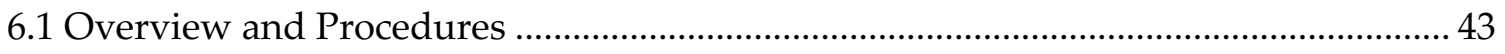

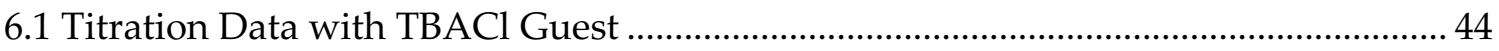

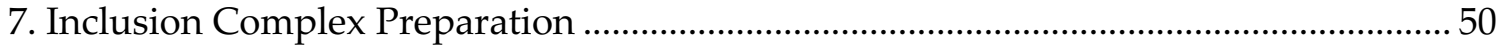

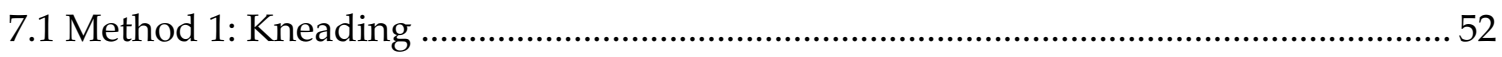

7.2 Method 2: Microwave Irradiation (Minimum Solvent) ................................................... 53

7.3 Method 3: Microwave Irradiation (2.5 mL of Solvent) ................................................... 53

8. Proton NMR Spectroscopy Analysis of Inclusion Complexes ....................................... 54

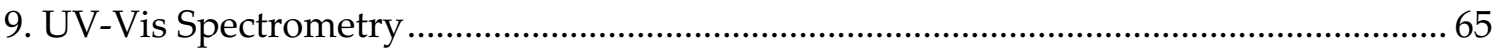

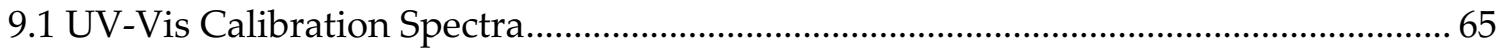

9.2 UV-Vis Quantification of Transporter Mass Percentage .................................................. 73

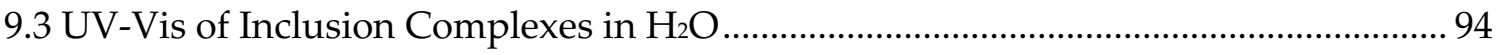

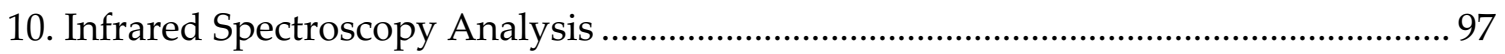

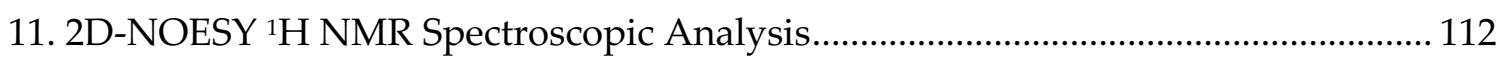


12. $\mathrm{Cl}^{-} / \mathrm{NO}_{3}{ }^{-}$Exchange Assay - Hill Analysis

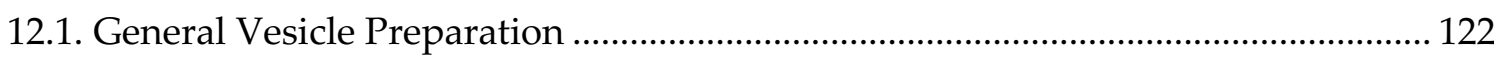

12.2. Electrode Calibration and Conversion of Raw Data ................................................. 122

12.3. Dose-Response Hill Analysis Assay Conditions ........................................................ 123

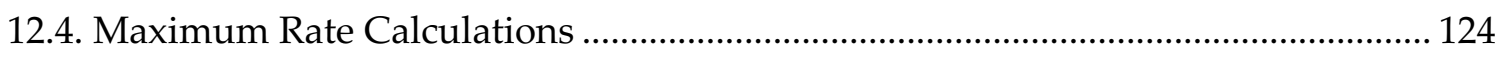

12.5. $\mathrm{Cl}^{-} / \mathrm{NO}_{3}{ }^{-}$Exchange Assay - Efflux and Hill Plots .................................................. 125 


\section{General Remarks}

All chemicals and solvents were purchased from commercials suppliers and used without further purification, unless stated otherwise. Deuterated solvents for NMR were purchased from Cambridge Isotope Laboratories. 1-Palmitoyl-2-oleoyl-sn-glycero-3-phosphocholine (POPC) was purchased from Avanti Lipids or Corden Pharma. Anhydrous solvents were collected from an Inert Corp PureSolv MD7 solvent purification system. Deionised water was collected from a Merck Millipore Milli- $\mathrm{Q}^{\mathrm{TM}}$ reference ultrapure water purification system.

NMR spectra ( ${ }^{1} \mathrm{H}$ NMR and ${ }^{13} \mathrm{C}$ NMR and 2D NOESY NMR) were collected on a Bruker AVIII 500 or a Bruker Avance DPX 400. NMR titrations were performed on the same instruments. Chemical shifts $(\delta)$ were reported in ppm and calibrated using the residual solvent peak of either DMSO- $d_{6}$, acetonitrile- $d_{3}$, or chloroform- $d$. Spin multiplicities were abbreviated to $\mathrm{s}=$ singlet, $\mathrm{d}=$ doublet $\mathrm{t}=$ triplet, $\mathrm{q}=$ quartet, $\mathrm{m}=$ multiplet and $\mathrm{br}=$ broad.

Melting points were recorded manually using a Standford Research Systems Optimelt machine, and data were reported as a range $\left({ }^{\circ} \mathrm{C}\right)$. Low resolution mass spectrometry (LR-MS) was performed with a Bruker amazon SL mass spectrometer using electrospray ionisation or APCI. High resolution mass spectra (HR-MS) were recorded on a Bruker Apex II Fourier Transform Ion Cyclotron Resonance (FTICR) mass spectrometer with a $7.0 \mathrm{~T}$ magnet, fitted with an off-axis analytic electrospray source with quadrupole mass analyser, and are reported as $\mathrm{m} / \mathrm{z}$ (relative intensity).

Infrared absorption spectra were recorded on a Bruker Alpha-E FT-IR spectrometer using attenuated total reflection (ATR) for the compounds and complexes as solids. Notable vibrational wavenumbers are labelled. UV-Vis spectra were recorded on a Cary 4000 UV-Vis spectrophotometer at $298 \mathrm{~K}$ using a $1 \mathrm{~cm}$ quartz cuvette, equipped with stirring multicell holder. The ISE assay experiments were conducted on the Fisherbrand ${ }^{\mathrm{TM}}$ Accumet $^{\mathrm{TM}}$ Chloride Combination Electrode. 


\section{Synthetic Overview of Compounds 1-6.}

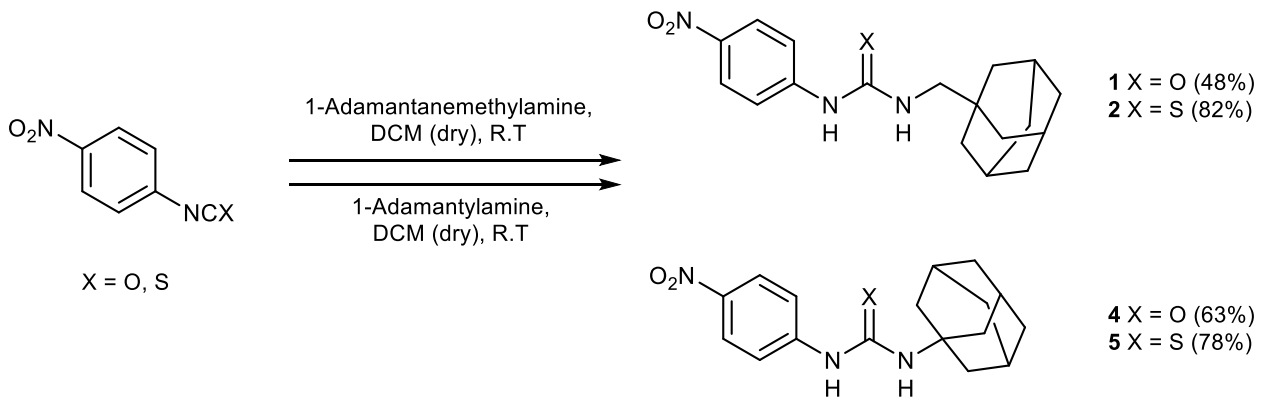

Scheme S1. The general synthesis of urea and thiourea compounds $1,2,4$, and 5 via condensation reaction between the relevant 4-nitrophenyl iso(thio)cyanate and adamantaneappended amine derivative.

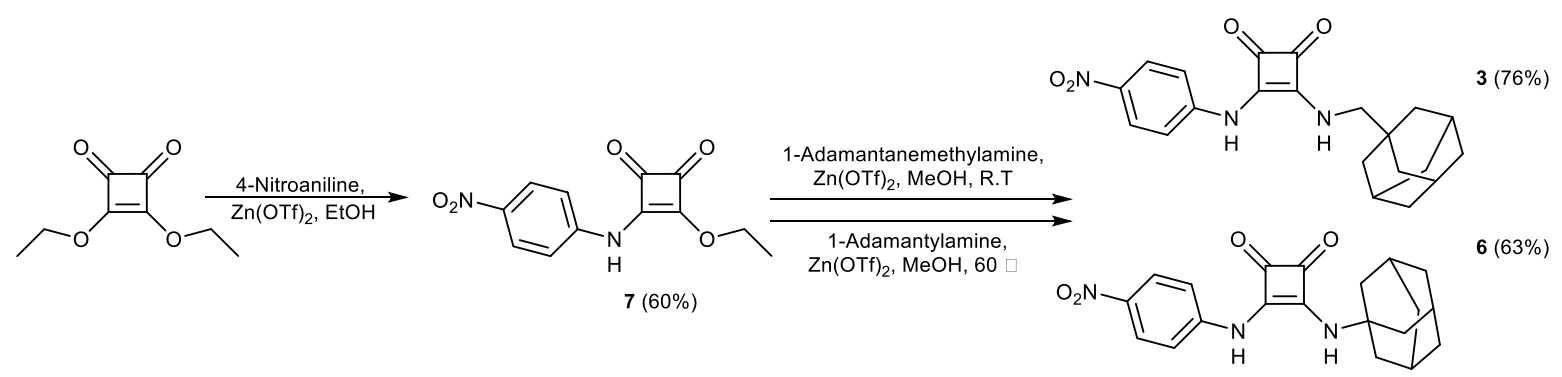

Scheme S2. The general synthesis of intermediate 7 and the final adamantyl-appended squaramide compounds 3 and 6 .

\section{Synthesis}

\section{1-(1-adamantylmethyl)-3-(4-nitrophenyl)urea (1)}<smiles>O=C(NCC12CC3CC(CC(C3)C1)C2)Nc1ccc([N+](=O)[O-])cc1</smiles>

4-nitrophenyl isocyanate $(547 \mathrm{mg}, 3.34 \mathrm{mmol})$ was dissolved in dry DCM (15 mL) before 1adamantyl methylamine $(0.53 \mathrm{~mL}, 3.04 \mathrm{mmol})$ was added dropwise to the solution. This was left to stir at R.T for $12 \mathrm{~h}$. A precipitate formed, which was identified as the symmetrical bis4-nitrophenylurea side-product. This was filtered off, and the solvent was removed from the 
filtrate in vacuo. The oily residue was subjected to column chromatography (30:70 ethyl acetate:hexane eluent) to yield a light yellow fluffy solid (480 mg, $1.46 \mathrm{mmol}, 48 \%$ ).

$\mathbf{M P}=219-222^{\circ} \mathrm{C}$

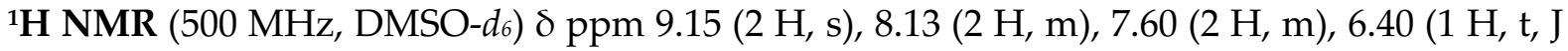
6.1), 2.83 (2 H, d, J 6.1), 1.94 (3 H, s), 1.68 (3 H, d, J 11.9), 1.59 (3 H, d, J 10.8), 1.46 (6 H, d, J 2.8); ${ }^{13}$ C NMR (101 MHz, DMSO-d6) $\delta$ ppm 155.1, 147.7, 140.8, 125.6, 117.1, 51.2, 37.0, 34.0, 28.1.

LR-MS $\left(\mathrm{ESI}^{+}\right) \mathrm{m} / \mathrm{z} 352.2[\mathrm{M}+\mathrm{Na}]^{+}, 681.38[2 \mathrm{M}+\mathrm{Na}]^{+}$; HR-MS (ESI ${ }^{+}$) calcd for $\mathrm{C}_{18} \mathrm{H}_{23} \mathrm{~N}_{3} \mathrm{O}_{3}$ $[\mathrm{M}+\mathrm{Na}]^{+}:$352.17394, found $\mathrm{m} / \mathrm{z} 352.16314$.

\section{1-(1-adamantylmethyl)-3-(4-nitrophenyl)thiourea (2)}

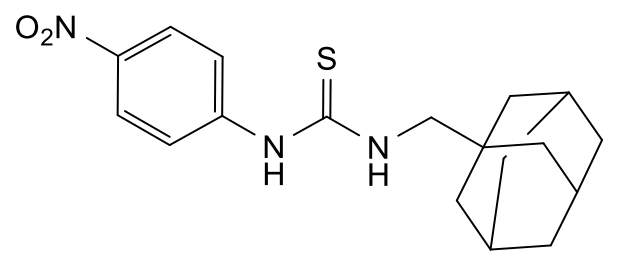

4-Nitrophenyl isothiocyanate (109 mg, $0.61 \mathrm{mmol})$ was dissolved in dry DCM (15 mL) before 1-adamantyl methylamine $(0.11 \mathrm{~mL}, 0.61 \mathrm{mmol})$ was added dropwise to the solution. This was left to stir at R.T for $12 \mathrm{~h}$. Next, the solvent was removed in vacuo and the residue subjected to column chromatography (30:70 ethyl acetate:hexane as the eluent). The solvent was removed in vacuo from the collected fraction and the resultant orange oil was dried in vacuo to yield a yellow solid (173 $\mathrm{mg}, 0.50 \mathrm{mmol}, 82 \%)$.

$\mathbf{M P}=199-202^{\circ} \mathrm{C}$

${ }^{1} \mathrm{H}$ NMR (500 MHz, DMSO-d $)$ d ppm $10.15(1 \mathrm{H}, \mathrm{s}), 8.17$ (2 H, d, J 9.2), 8.12 (1 H, s), $7.94(2 \mathrm{H}$, d, J 9.2), 3.29 (2 H, d, J 5.3), 1.96 (2 H, s), 1.69 (3 H, d, J 12.1), 1.62 (3 H, d, J 10.8), 1.54 (6 H, d, J 2.9); ${ }^{13}$ C NMR (101 MHz, DMSO-d 6 ) $\delta$ ppm 181.0, 147.1, 142.1, 124.9, 120.6, 55.8, 36.9, 34.2, 28.1. LR-MS (ESI $\left.{ }^{-}\right) \mathrm{m} / \mathrm{z} 344.23[\mathrm{M}-\mathrm{H}]^{-}, 725.47[\mathrm{M}+\mathrm{Cl}]^{-} ;$HR-MS $\left(\mathrm{ESI}^{+}\right)$calcd for $\mathrm{C}_{18} \mathrm{H}_{23} \mathrm{~N}_{3} \mathrm{O}_{2} \mathrm{~S}$ $[\mathrm{M}+\mathrm{Na}]^{+}: 368.14032$, found $m / z$ 368.14031.

3-ethoxy-4((4-nitrophenyl)amino)cyclobutene-1,2-dione (7) 


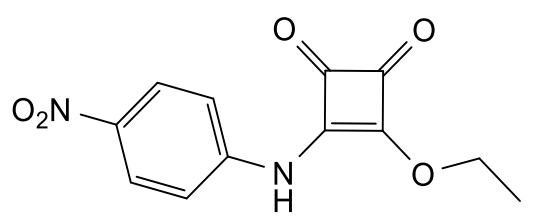

4-nitroaniline ( $1 \mathrm{~g}, 7.25 \mathrm{mmol})$ and zinc triflate $(500 \mathrm{mg}$, $1.38 \mathrm{mmol})$ were dissolved in ethanol $(50 \mathrm{~mL})$ and stirred, before diethylsquarate $(1 \mathrm{~mL}, 6.76 \mathrm{mmol})$ was added dropwise. The reaction flask was covered in foil and left to stir overnight. A precipitate formed which was filtered and washed with cold ethanol $(2 \times 25 \mathrm{~mL})$ and dried in vacuo overnight to leave a pure orange solid (733 mg, $2.80 \mathrm{mmol}, 41 \%$ ). The ${ }^{1} \mathrm{H}-\mathrm{NMR}$ and LR-MS spectra were found to be consistent with literature spectra.

${ }^{1}$ H NMR (400 MHz, DMSO-d6) ठ ppm 11.20 (1 H, s), 8.21 (2 H, d, J 9.2), 7.59 (2 H, d, J 9.2), 4.80 (2 H, d, J 7.1), 3.17 (3 H, 7, J 7.1), ethanol solvent peaks are also visible.

LR-MS (ESI-) $m / z 260.79$ [M-H] $]^{-}$.

\section{$N$-(1-adamantylmethyl)- $N^{\prime}$-(4-nitrophenyl)squaramide (3)}

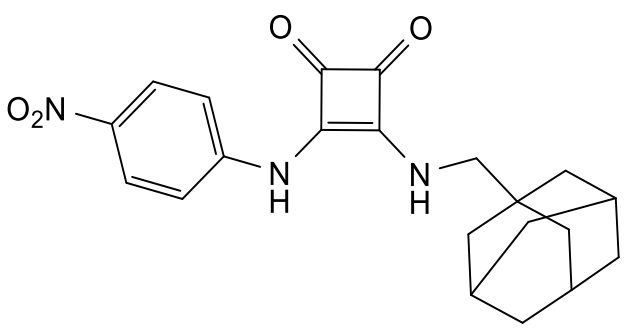

Compound 7 (400 mg, $1.52 \mathrm{mmol})$ and zinc triflate $(111 \mathrm{mg}, 0.3 \mathrm{mmol})$ were dissolved as much as possible in methanol $(35 \mathrm{~mL})$ and stirred. Next, 1-adamantylmethylamine $(0.3 \mathrm{~mL}, 1.67$ $\mathrm{mmol}$ ) was added dropwise and the solution immediately changed colour from orange to dark red. The reaction was left overnight before a precipitate formed. This was filtered, washed with cold methanol $(2 \times 50 \mathrm{~mL})$, and dried in vacuo to yield a pure dark red solid (442 $\mathrm{mg}, 1.16 \mathrm{mmol}, 76 \%)$.

$\mathbf{M P}=+300{ }^{\circ} \mathrm{C}$

${ }^{1} \mathrm{H}$ NMR (500 MHz, DMSO-d6) $\delta$ ppm 9.96 (1H, br), $8.21(2 \mathrm{H}, \mathrm{d}, \mathrm{J} 8.8), 7.84(1 \mathrm{H}, \mathrm{s}), 7.62(2 \mathrm{H}$, d, J 8.8), 1.97 (3 H, s), 1.76 - 1.57 (6 H, m), $1.50(6 \mathrm{H}, \mathrm{s})$ [Methyl proton signal hidden beneath water peak]; ${ }^{13}$ C NMR (101 MHz, DMSO-d6) $\delta$ ppm 185.1, 180.1, 170.7, 162.0, 145.4, 141.3, 125.6, $117.6,55.3,36.3,27.5$. 
LR-MS (ESI-) m/z 379.95 [M-H] $]^{-}$; HR-MS (ESI ${ }^{+}$) calcd for $\mathrm{C}_{21} \mathrm{H}_{23} \mathrm{~N}_{3} \mathrm{O}_{4}[\mathrm{M}+\mathrm{H}]^{+}:$382.17613, found $m / z 382.17583$.

\section{1-adamantyl-3-(4-nitrophenyl)urea (4)}<smiles>O=C(Nc1ccc([N+](=O)[O-])cc1)NC12CC3CC(CC(C3)C1)C2</smiles>

4-Nitrophenylisocyanate (412 mg, $2.52 \mathrm{mmol}$ ) was dissolved in dry DCM (15 mL). Separately, 1-adamantylamine (190 mg, $1.26 \mathrm{mmol})$ was dissolved in dry DCM (15 mL) and added dropwise to the solution. This was left to stir at R.T overnight before a yellow precipitate was filtered off, which proved to be the mono-urea symmetrical 4-nitrophenyl side product. The solvent was removed in vacuo and the residue purified using silica gel column chromatography (30:70 ethyl acetate:hexane eluent). The solvent was removed in vacuo to yield a fluffy pale-yellow solid (252 mg, $0.80 \mathrm{mmol}, 63 \%)$.

$\mathbf{M P}=228-231^{\circ} \mathrm{C}$

${ }^{1}$ H NMR (400 MHz, DMSO-d6) d ppm $9.01(1 \mathrm{H}, \mathrm{s}), 8.11$ (2 H, d, J 9.3), 7.56 (2H, d, J 9.2), 6.15 $(1 \mathrm{H}, \mathrm{s}), 2.03(3 \mathrm{H}, \mathrm{s}), 1.95(6 \mathrm{H}, \mathrm{s}), 1.64(6 \mathrm{H}, \mathrm{s}) ;{ }^{13} \mathrm{C}$ NMR (101 MHz, DMSO-d 6$) \delta$ ppm 153.0, $147.2,140.2,125.1,116.5,50.2,41.4,35.9,28.8$.

LR-MS (ESI $\left.{ }^{+}\right)$m/z 313.89 [M-H] $]^{-}$; HR-MS (ESI ${ }^{+}$calcd for $\mathrm{C}_{17} \mathrm{H}_{21} \mathrm{~N}_{3} \mathrm{O}_{3}[\mathrm{M}+\mathrm{Na}]^{+}:$: 338.14751, found $\mathrm{m} / \mathrm{z} 338.14729$.

\section{1-adamantyl-3-(4-nitrophenyl)thiourea (5)}<smiles>O=[N+]([O-])c1ccc(NC(=S)NC23CC4CC(CC(C4)C2)C3)cc1</smiles> 
4-Nitrophenylisothiocyanate $(160 \mathrm{mg}, 1.46 \mathrm{mmol})$ was dissolved in dry DCM (15 mL). Separately, 1-adamantylamine (200 mg, $1.32 \mathrm{mmol})$ was dissolved in dry DCM (15 mL) and added dropwise to the solution. This was left to stir at R.T for $12 \mathrm{~h}$. A precipitate formed was filtered and washed with DCM $(3 \times 30 \mathrm{~mL})$ to give the pure white product. Next, the solvent was removed in vacuo and the residue subjected to column chromatography (10:90 ethyl acetate:hexane as the eluent). The solvent was removed in vacuo from the collected fraction and the solid was dried in vacuo to yield a white solid (312 $\mathrm{mg}, 0.94 \mathrm{mmol}, 71 \%$ ).

$\mathbf{M P}=187-190^{\circ} \mathrm{C}$

${ }^{1}$ H NMR (500 MHz, DMSO-d6) $\delta$ ppm 9.89 (1 H, s), 8.15 (2 H, d, J 9.2), 7.82 (2 H, d, J 9.2), 7.78 (1 H, s), 2.25 (6 H, s), 2.07 (3 H, s), $1.65(6 \mathrm{H}, \mathrm{s}){ }^{13} \mathrm{C}$ NMR (101 MHz, DMSO-d 6$) \delta$ ppm 178.0, $146.6,141.5,124.4,120.3,53.9,35.9,28.9$.

LR-MS (ESI $\left.{ }^{+}\right) \mathrm{m} / \mathrm{z} 332.18[\mathrm{M}+\mathrm{H}]^{+}, 685.23[2 \mathrm{M}+\mathrm{Na}]^{+}$; HR-MS (ESI ${ }^{+}$calcd for $\mathrm{C}_{17} \mathrm{H}_{21} \mathrm{~N}_{3} \mathrm{O}_{2} \mathrm{~S}$ $[\mathrm{M}+\mathrm{Na}]^{+}:$354.12467, found $m / z$ 354.12456.

\section{$N$-(adamantyl)- $N^{\prime}-(4-n i t r o p h e n y l)$ squaramide (6)}

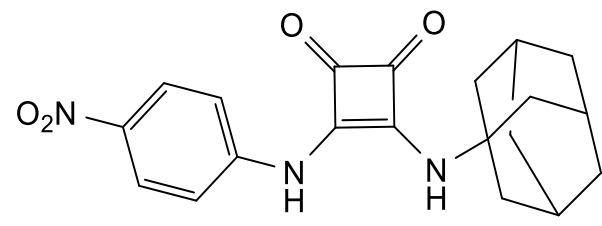

Compound 7 (400 mg, $1.52 \mathrm{mmol})$ and zinc triflate $(111 \mathrm{mg}, 0.3 \mathrm{mmol})$ were dissolved as much as possible in methanol (35 mL) and stirred. 1-adamantylamine (252 $\mathrm{mg}, 1.67 \mathrm{mmol})$ was added to the solution. Triethylamine $(1 \mathrm{~mL})$ was added to the reaction, which was left to stir overnight. A small amount of red precipitate formed which was identified as product. The solvent was removed from the reaction mixture in vacuo before the residue was subjected to column chromatography (2:98 methanol:dichloromethane eluent with 3\% TEA). The fractions containing pure compound were dried to leave an orange-red solid (346 mg, 0.94 mmol, 63\%).

$\mathbf{M P}=+300{ }^{\circ} \mathrm{C}$

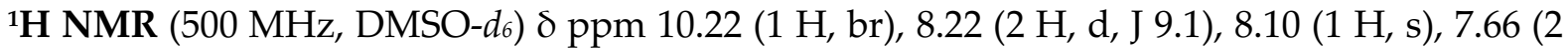
H, d, J 9.1), 2.11 (3 H, s), 1.99 (6 H, s), 1.66 (6 H, s); ${ }^{13}$ C NMR (101 MHz, DMSO-d6) ठ ppm 183.4, $179.6,170.2,163.2,145.2,141.5,125.6,117.7,53.1,42.4,35.2,28.9$. 
LR-MS (ESI-) $m / z 365.91[\mathrm{M}-\mathrm{H}]^{-} ;$HR-MS $\left(\right.$ESI $\left.^{+}\right)$calcd for $\mathrm{C}_{20} \mathrm{H}_{21} \mathrm{~N}_{3} \mathrm{O}_{4}[\mathrm{M}+\mathrm{H}]^{+}: 368.16048$, found $m / z 368.16020$.

\section{Characterisation Data}

Proton NMR and low-resolution mass spectrometry (LR-MS) are shown for each compound. Carbon NMR and high-resolution mass spectrometry (HR-MS) are shown for novel compounds.

1-(1-adamantylmethyl)-3-(4-nitrophenyl)urea (1)<smiles>O=C(NCC12CC3CC(CC(C3)C1)C2)Nc1ccc([N+](=O)[O-])cc1</smiles> 


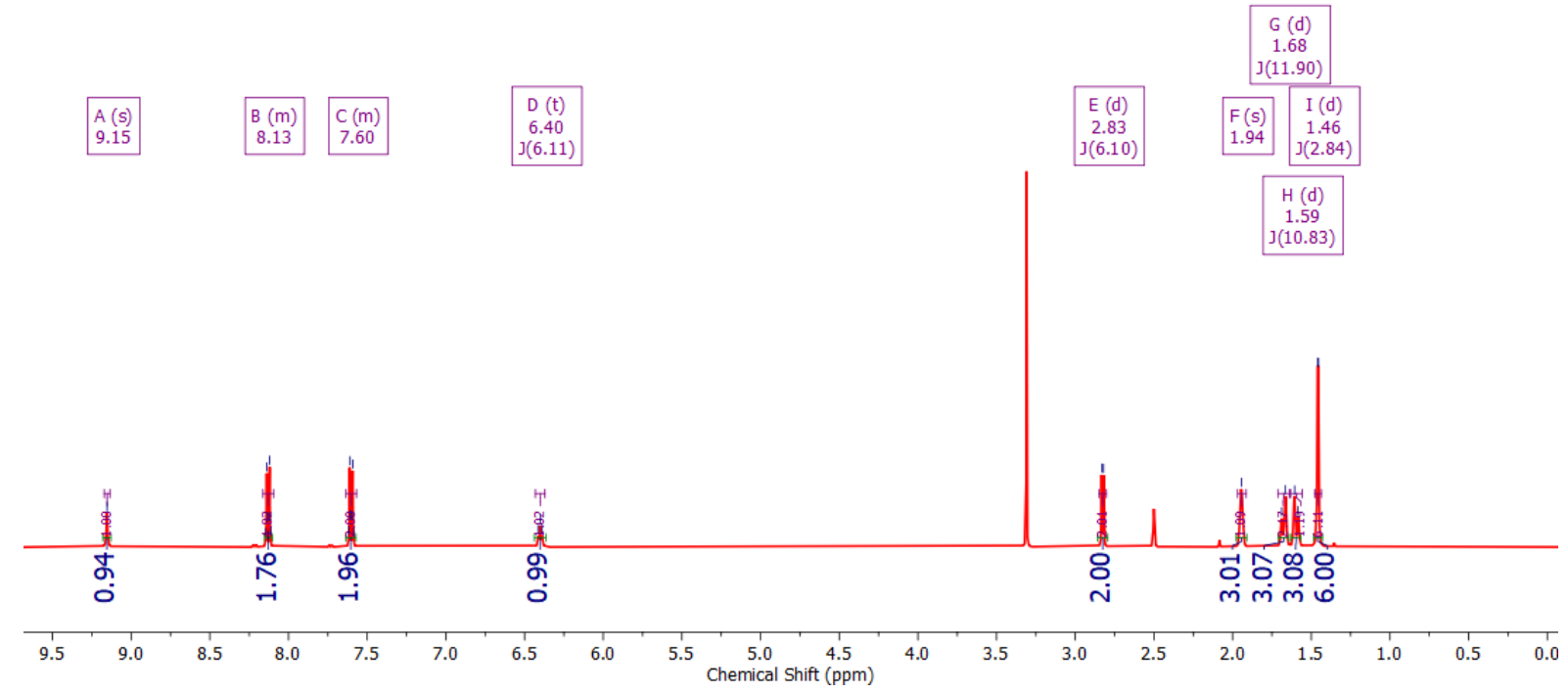

Figure S1. ${ }^{1} \mathrm{H}$ NMR (500 MHz) spectrum of 1-(1-adamantylmethyl)-3-(4-nitrophenyl)urea in DMSO- $d_{6}$ at $298 \mathrm{~K}$. 

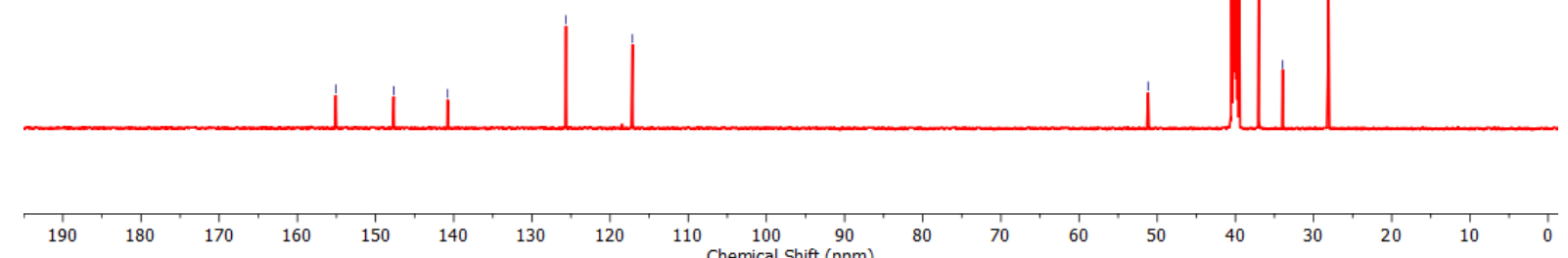

Figure S2. ${ }^{13} \mathrm{C}$ NMR (101 MHz) spectrum of 1-(1-adamantylmethyl)-3-(4-nitrophenyl)urea in DMSO- $d_{6}$ at $298 \mathrm{~K}$.

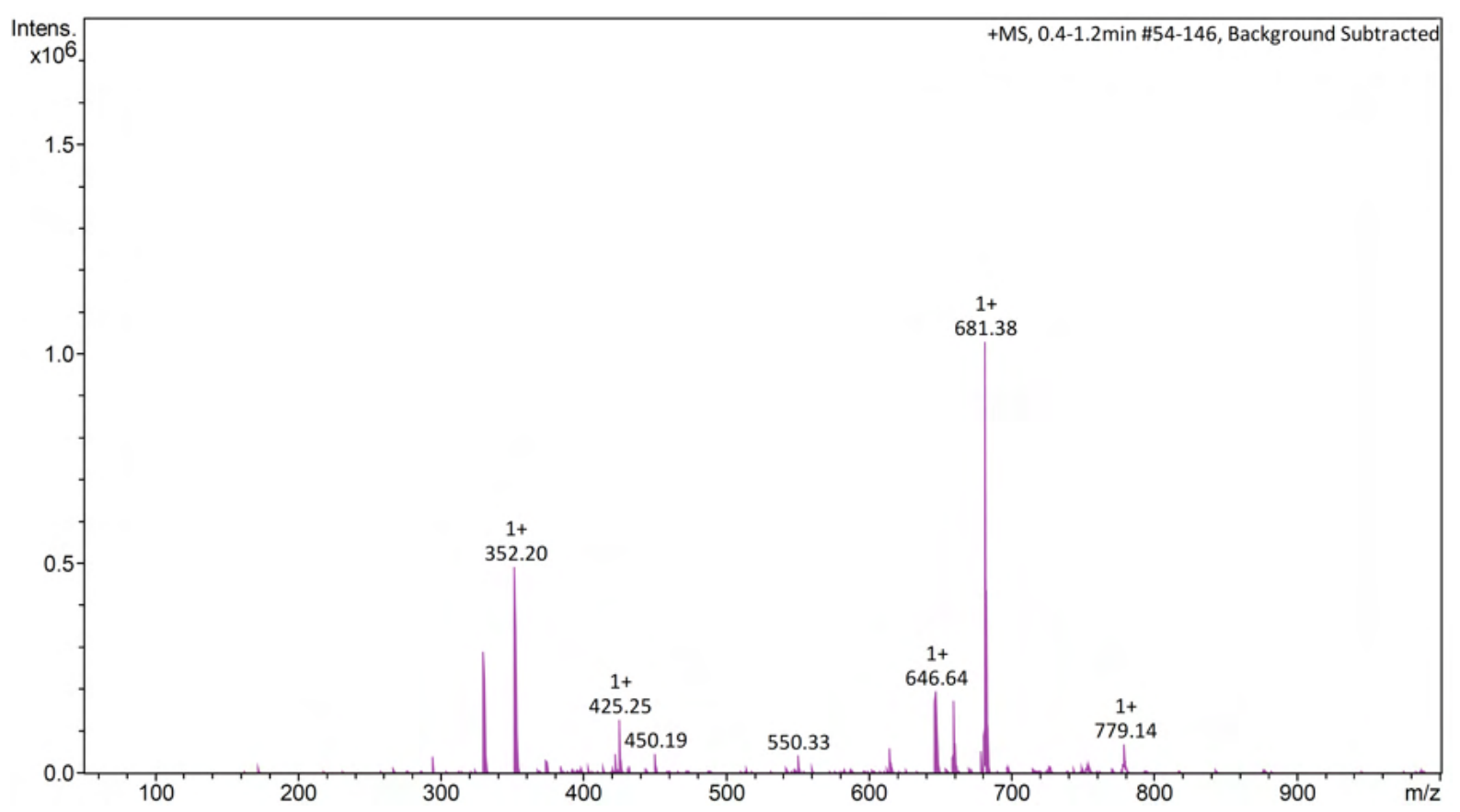

Figure S3. LR-MS (ESI+) spectrum of 1-(1-adamantylmethyl)-3-(4-nitrophenyl)urea. 


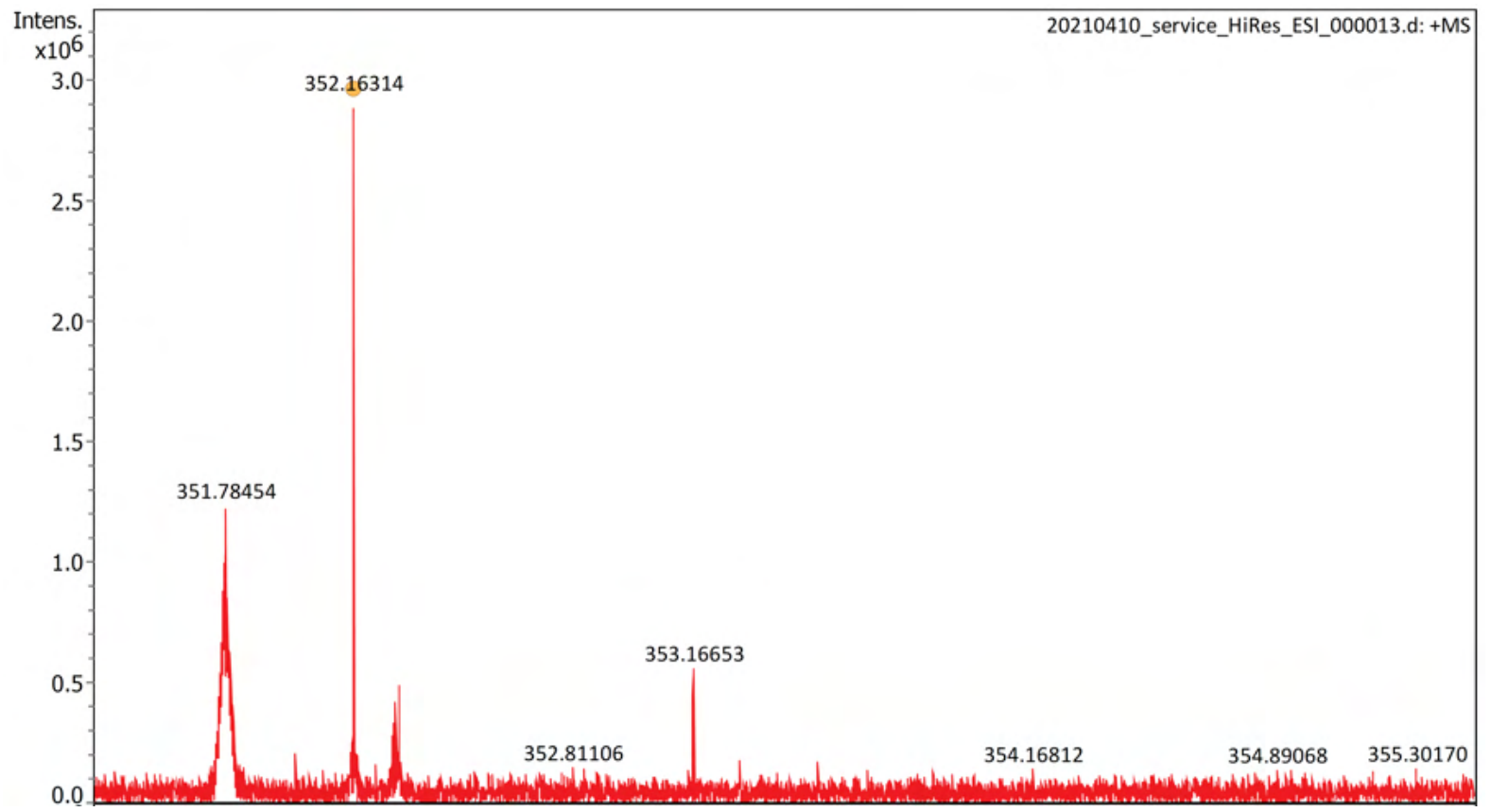

Figure S4. HR-MS (ESI ${ }^{+}$) spectrum of 1-(1-adamantylmethyl)-3-(4-nitrophenyl)urea. 
1-(1-adamantylmethyl)-3-(4-nitrophenyl)thiourea - (2)<smiles>O=[N+]([O-])c1ccc(NC(=S)NCC23CC4CC(CC(C4)C2)C3)cc1</smiles>

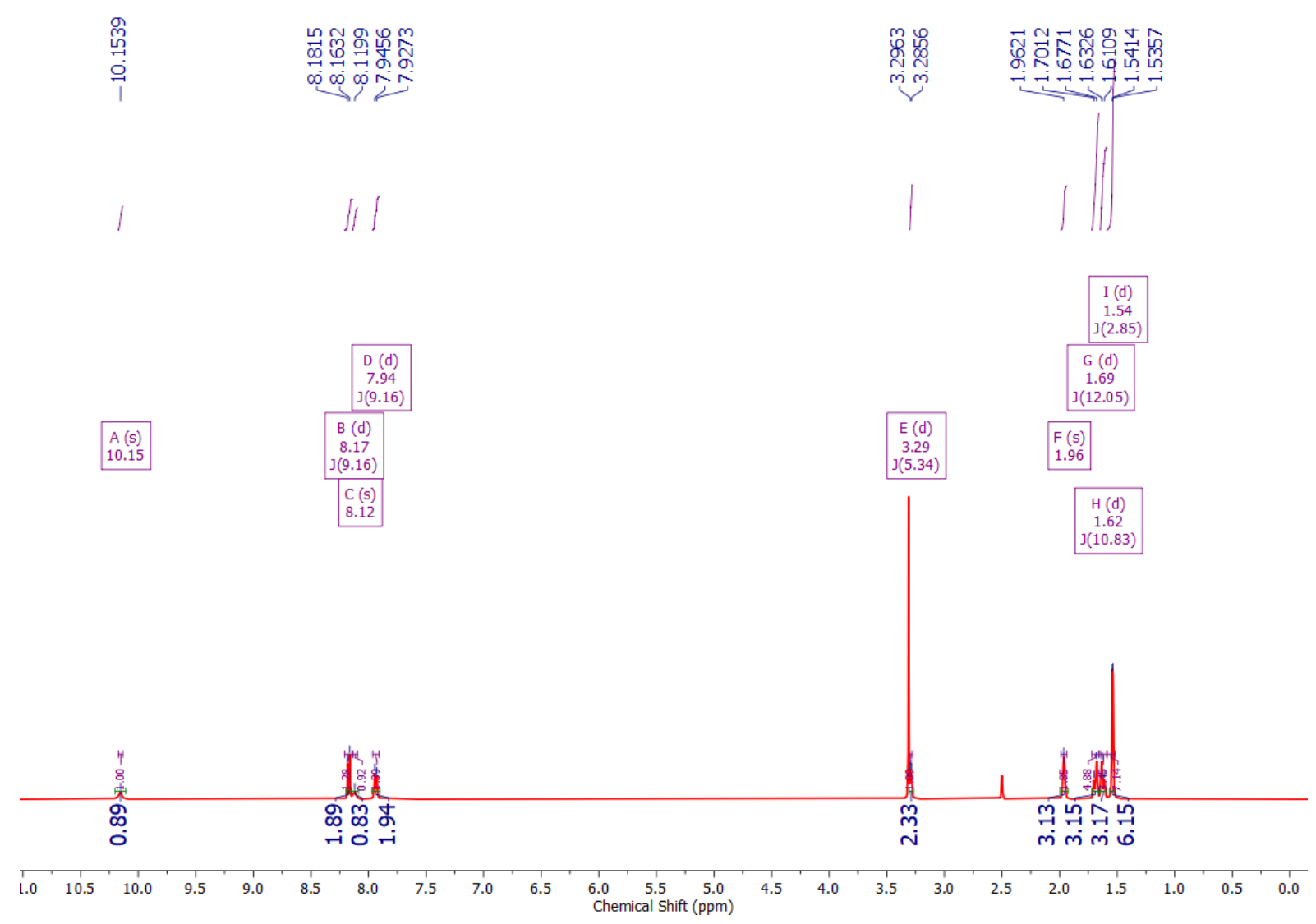

Figure S5. ${ }^{1} \mathrm{H}$ NMR (500 MHz) spectrum of 1-(1-adamantylmethyl)-3-(4-nitrophenyl)thiourea in DMSO- $d_{6}$ at $298 \mathrm{~K}$. 

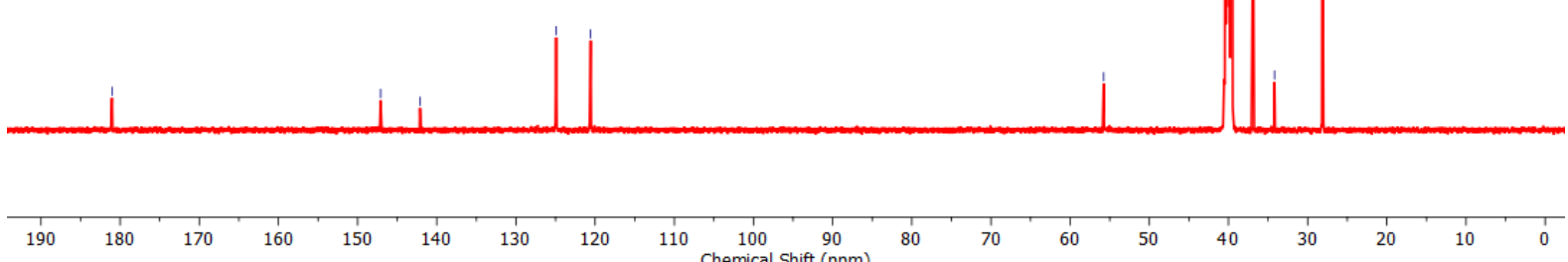

Figure S6. ${ }^{13} \mathrm{C}$ NMR (101 MHz) spectrum of 1-(1-adamantylmethyl)-3-(4-nitrophenyl)thiourea in DMSO- $d_{6}$ at $298 \mathrm{~K}$.

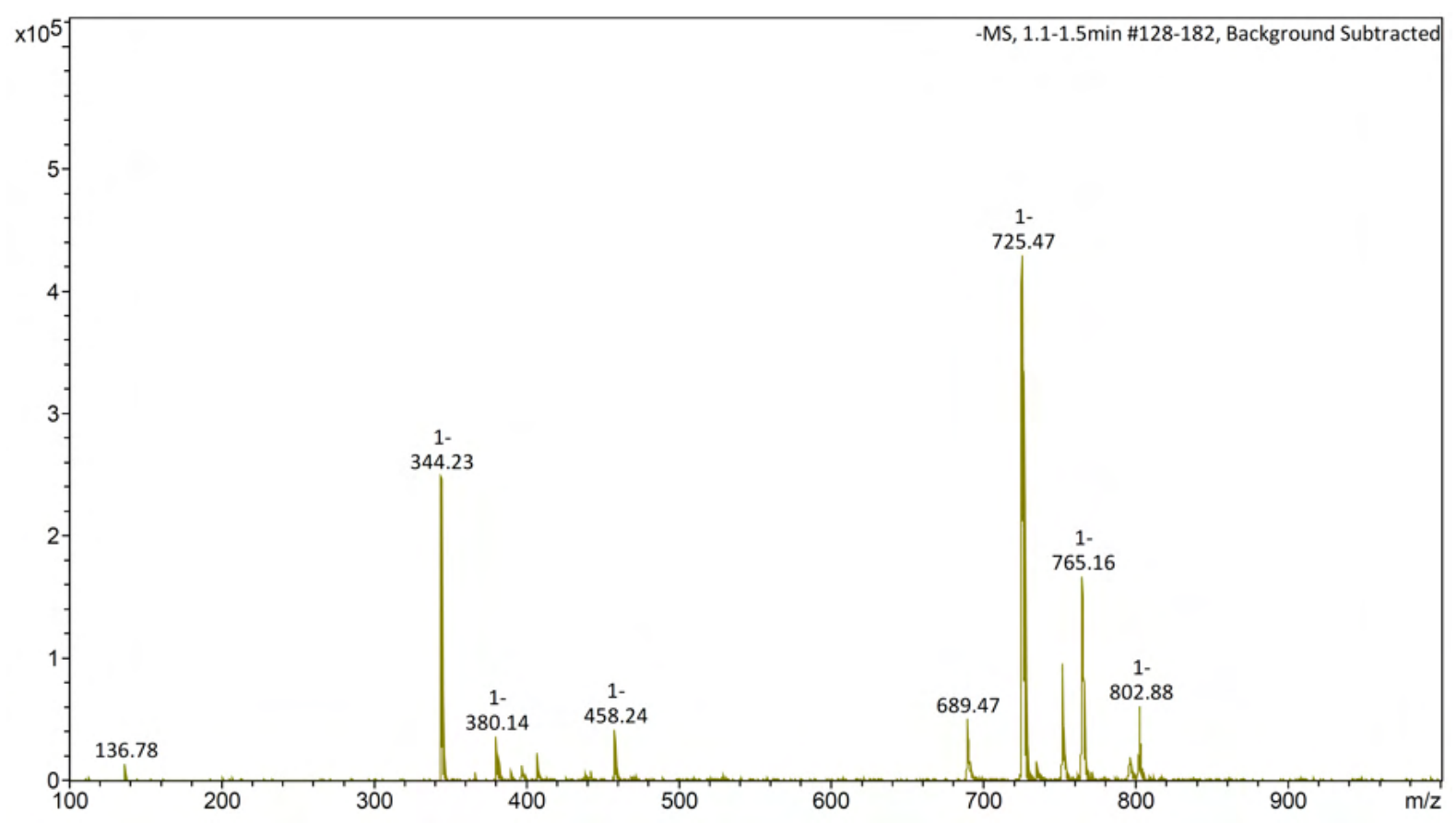

Figure S7. LR-MS (ESI-) spectrum of 1-(1-adamantylmethyl)-3-(4-nitrophenyl)thiourea. 


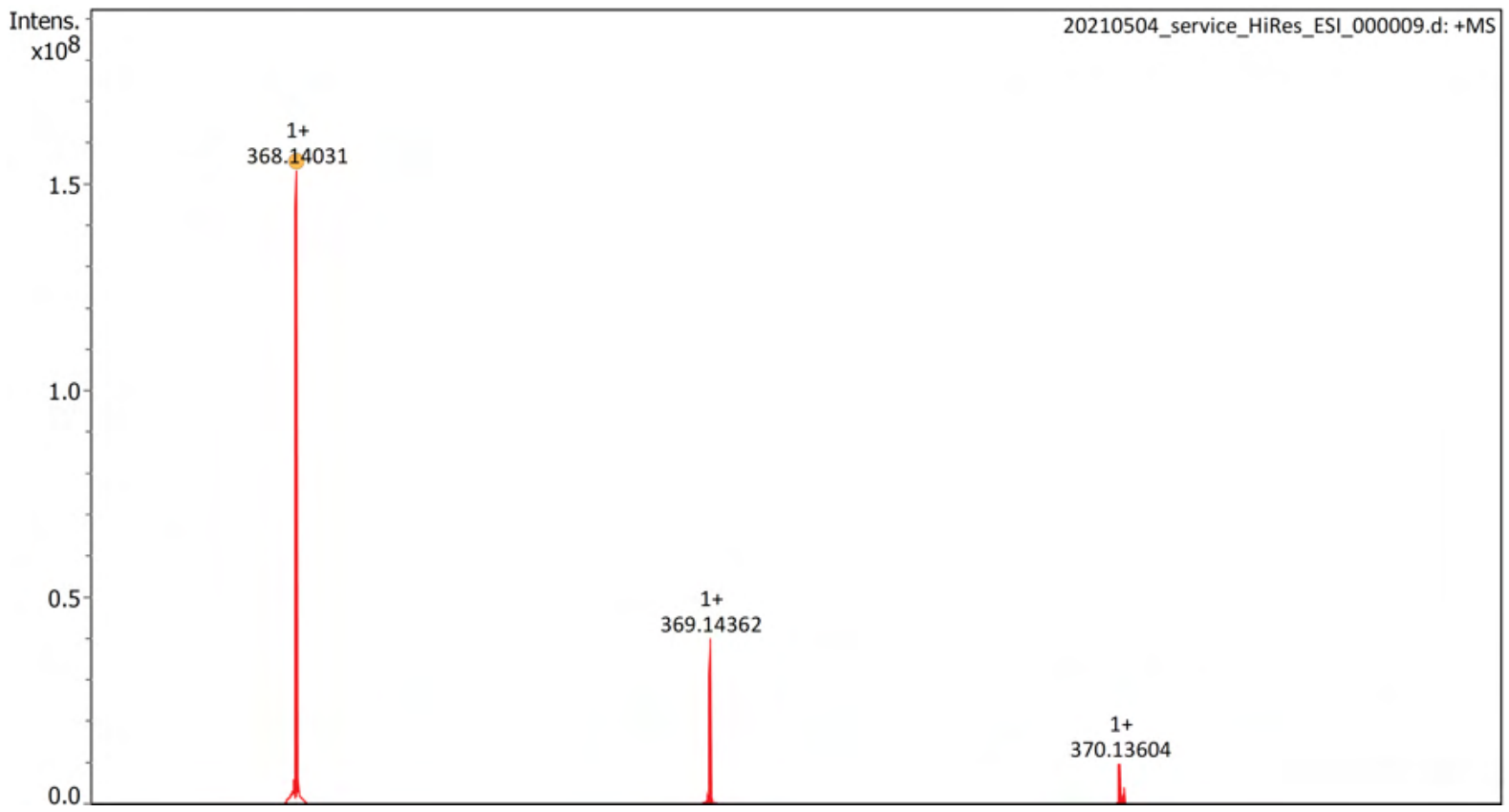

Figure S8. HR-MS (ESI ${ }^{+}$spectrum of 1-(1-adamantylmethyl)-3-(4-nitrophenyl)thiourea. 
3-ethoxy-4((4-nitrophenyl)amino)cyclobutene-1,2-dione (7)<smiles>CCOc1c(Nc2ccc([N+](=O)[O-])cc2)c(=O)c1=O</smiles>
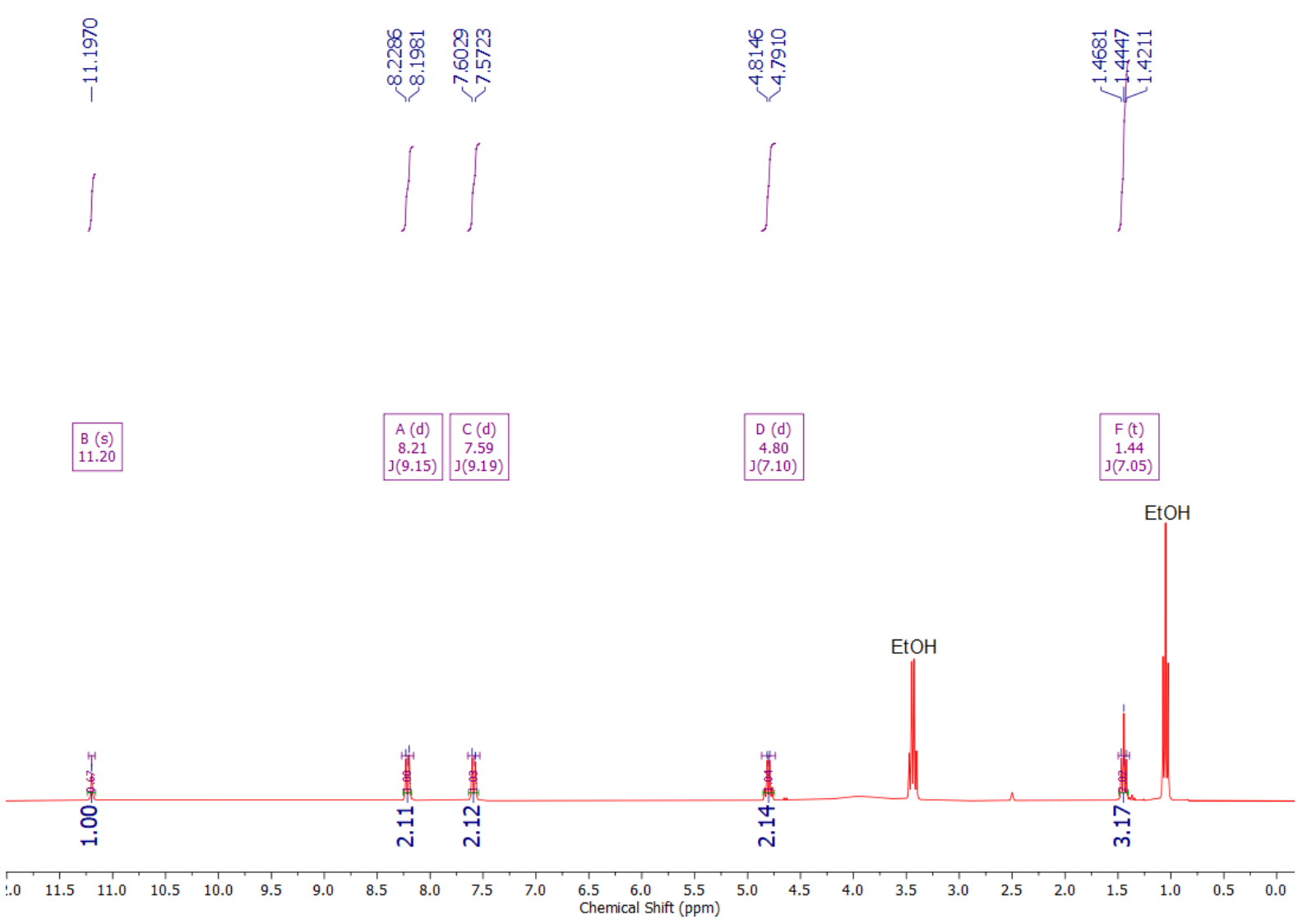

Figure S9. ${ }^{1} \mathrm{H}$ NMR (400 MHz) spectrum of 3-ethoxy-4((4-nitrophenyl)amino)cyclobutene-1,2dione in DMSO- $d_{6}$ at $298 \mathrm{~K}$. 


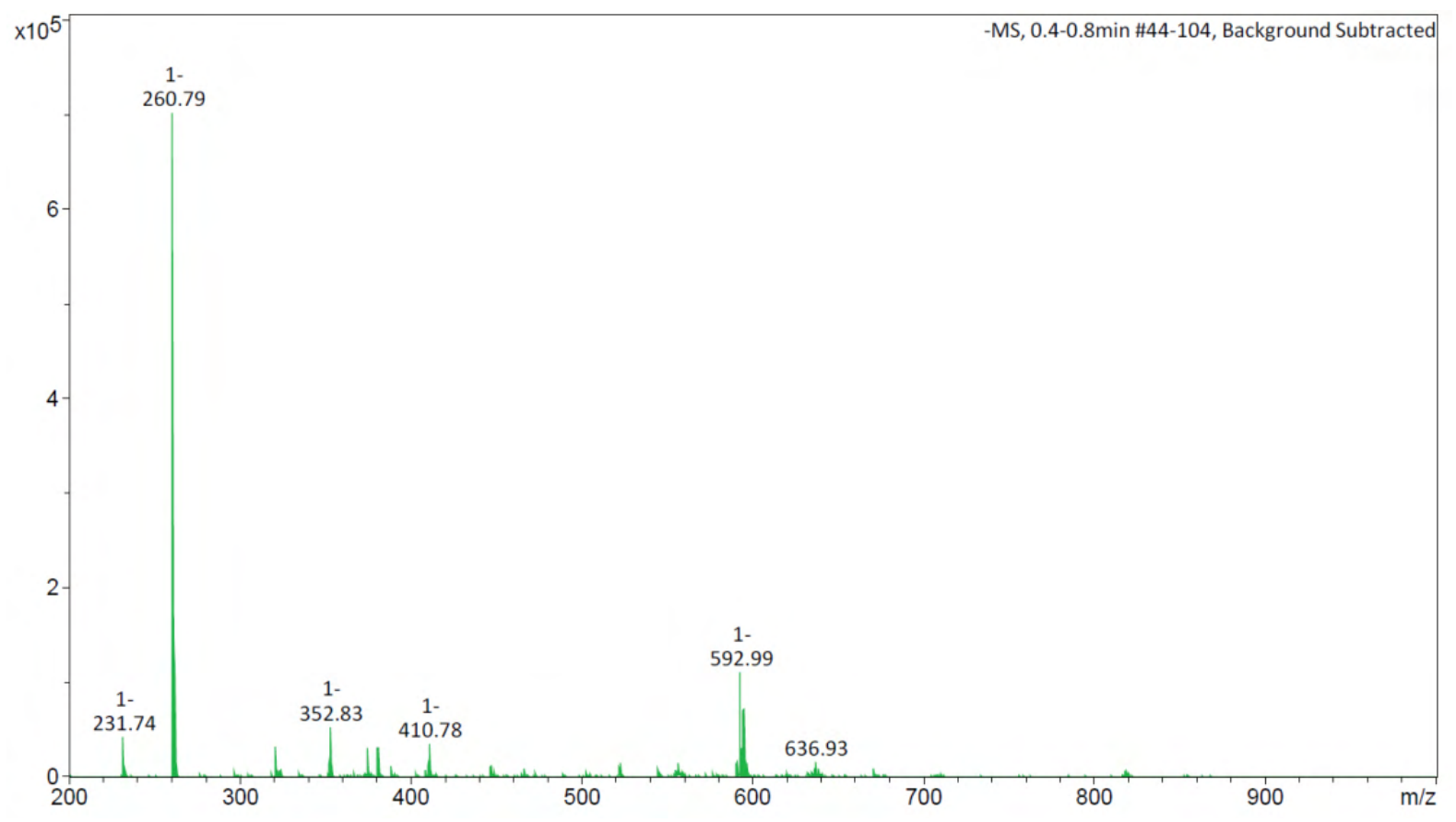

Figure S10. LR-MS (ESI-) spectrum of 3-ethoxy-4((4-nitrophenyl)amino)cyclobutene-1,2dione. 
$N$-(1-adamantylmethyl)- $N^{\prime}$-(4-nitrophenyl)squaramide (3)
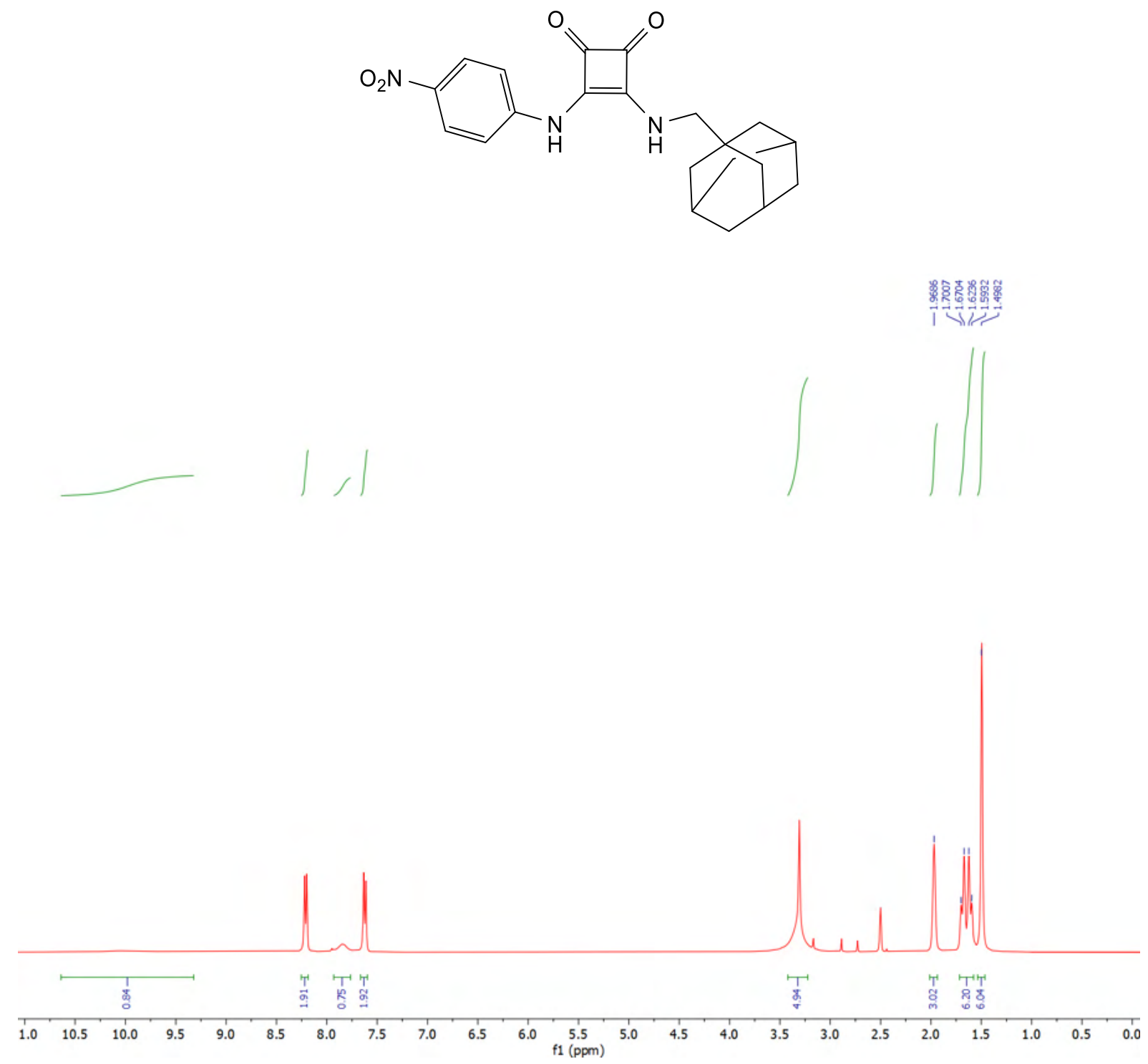

Figure S11. $\quad{ }^{1} \mathrm{H} \quad \mathrm{NMR} \quad(500 \quad \mathrm{MHz}) \quad$ spectrum of $\quad N$-(1-adamantylmethyl)- $N^{\prime}$-(4nitrophenyl)squaramide in DMSO- $d_{6}$ at $298 \mathrm{~K}$. 


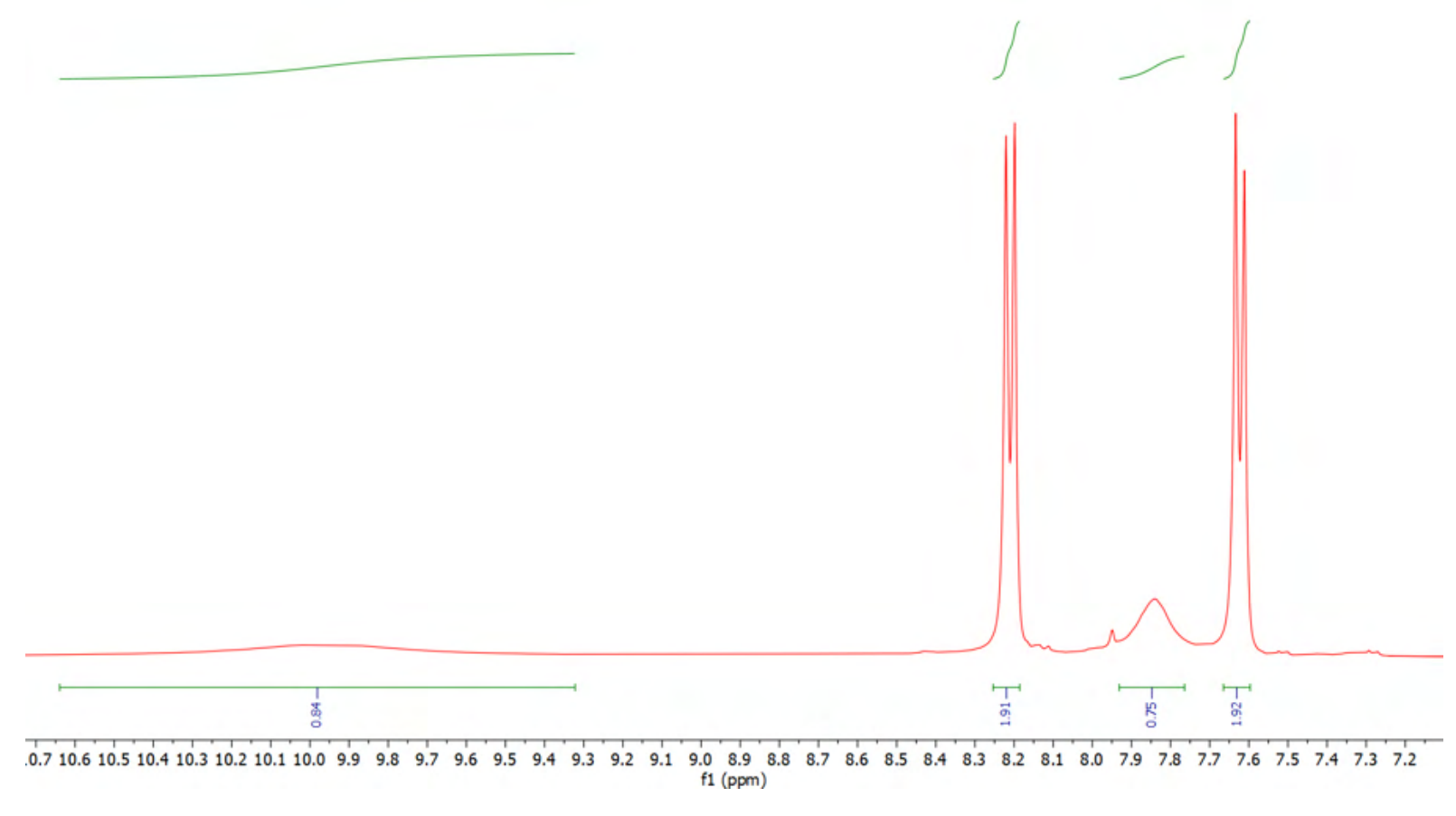

Figure S12. $\quad{ }^{1} \mathrm{H} \quad \mathrm{NMR} \quad(500 \mathrm{MHz}) \quad$ spectrum of $\quad N$-(1-adamantylmethyl)- $N^{\prime}-(4-$ nitrophenyl)squaramide in DMSO- $d_{6}$ at $298 \mathrm{~K}$. Zoomed in to display broad resonance attributed to the squaramide $\mathrm{N}-\mathrm{H}$ proton. 

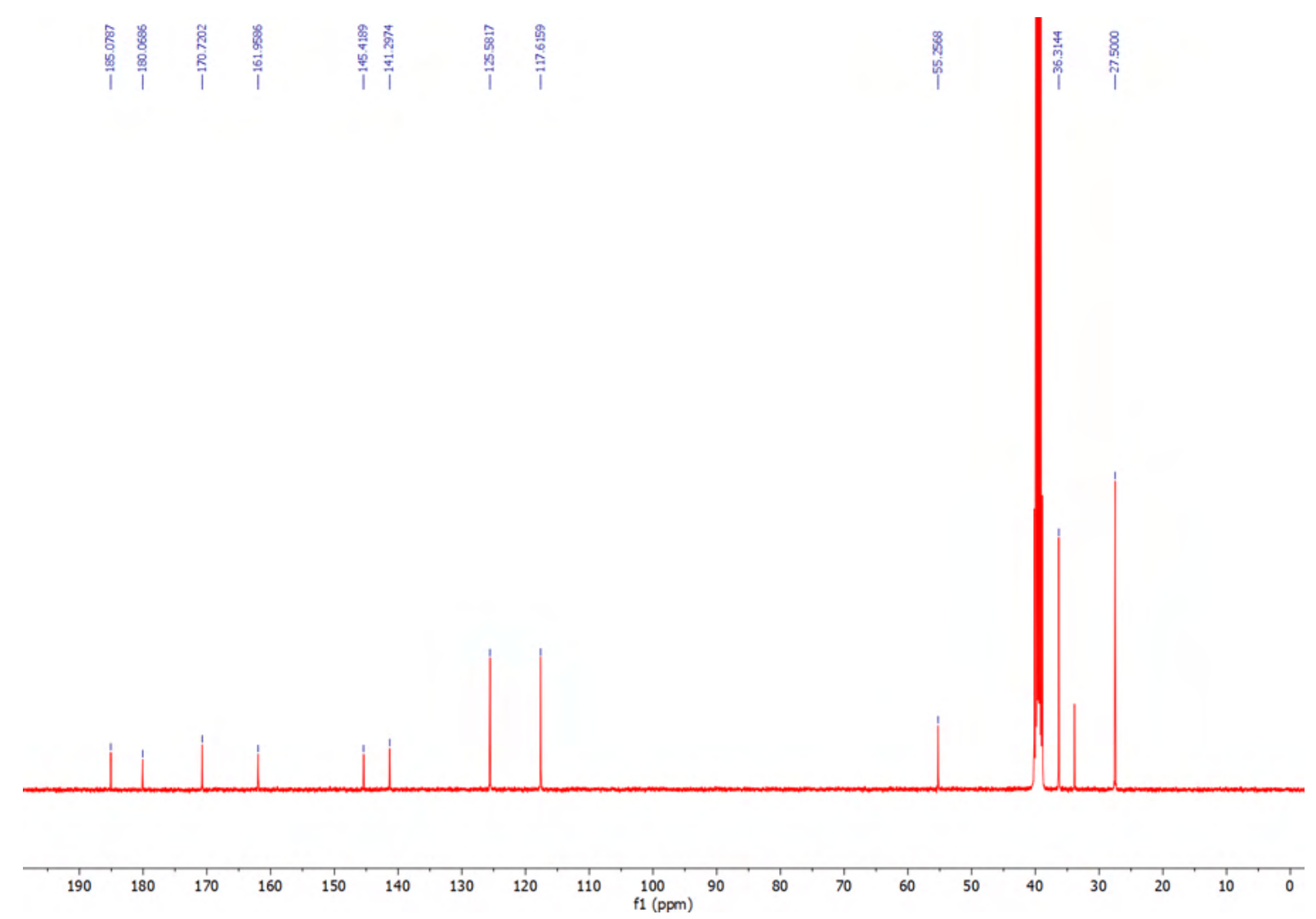

Figure S13. ${ }^{13} \mathrm{C}$ NMR (101 MHz) spectrum of $N$-(adamantyl)- $N^{\prime}$-(4-nitrophenyl)squaramide urea in DMSO- $d_{6}$ at $298 \mathrm{~K}$.

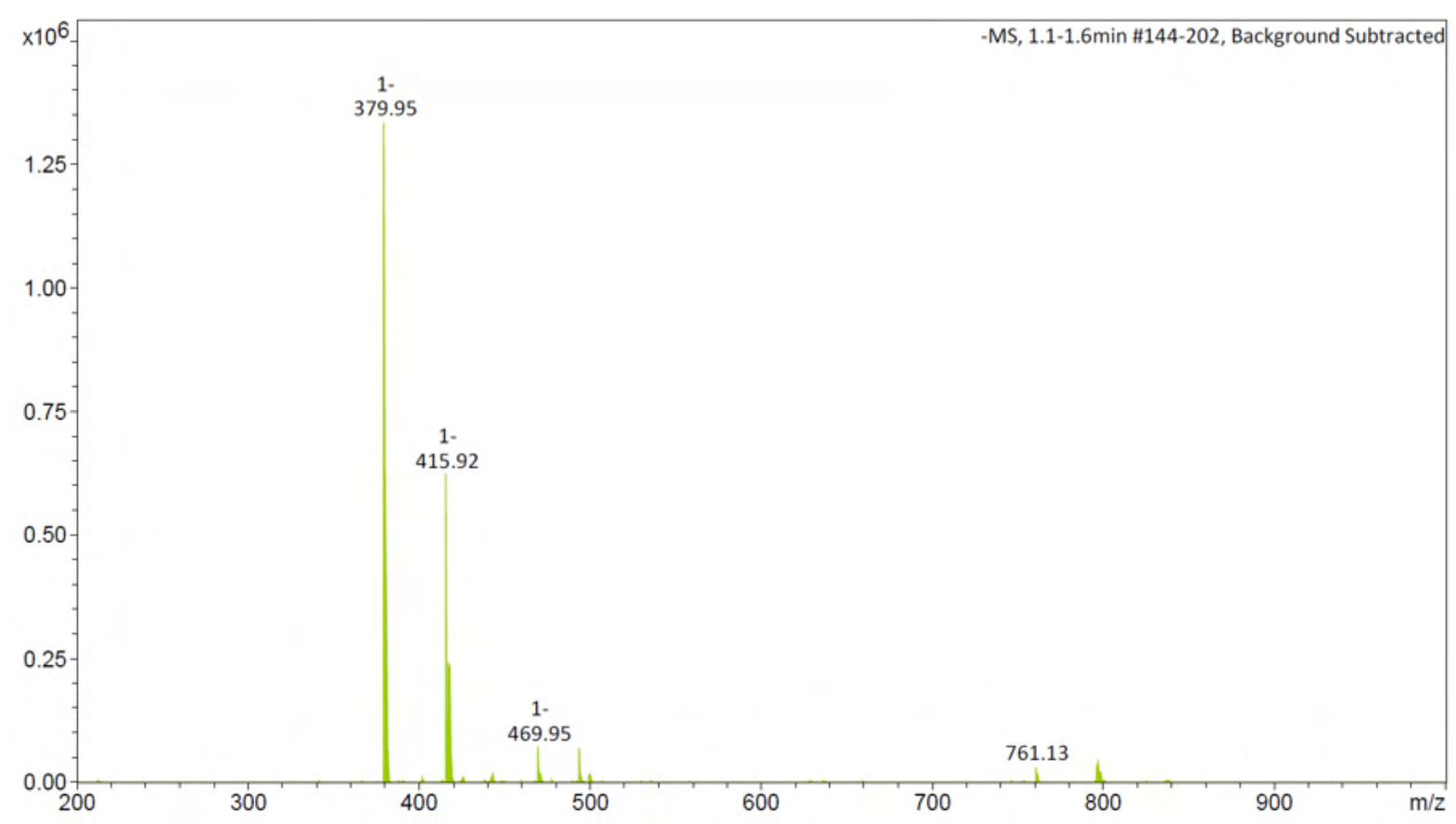

Figure S14. LR-MS (ESI $\left.{ }^{-}\right)$spectrum of $N$-(1-adamantylmethyl)- $N^{\prime}$-(4-nitrophenyl)squaramide. 


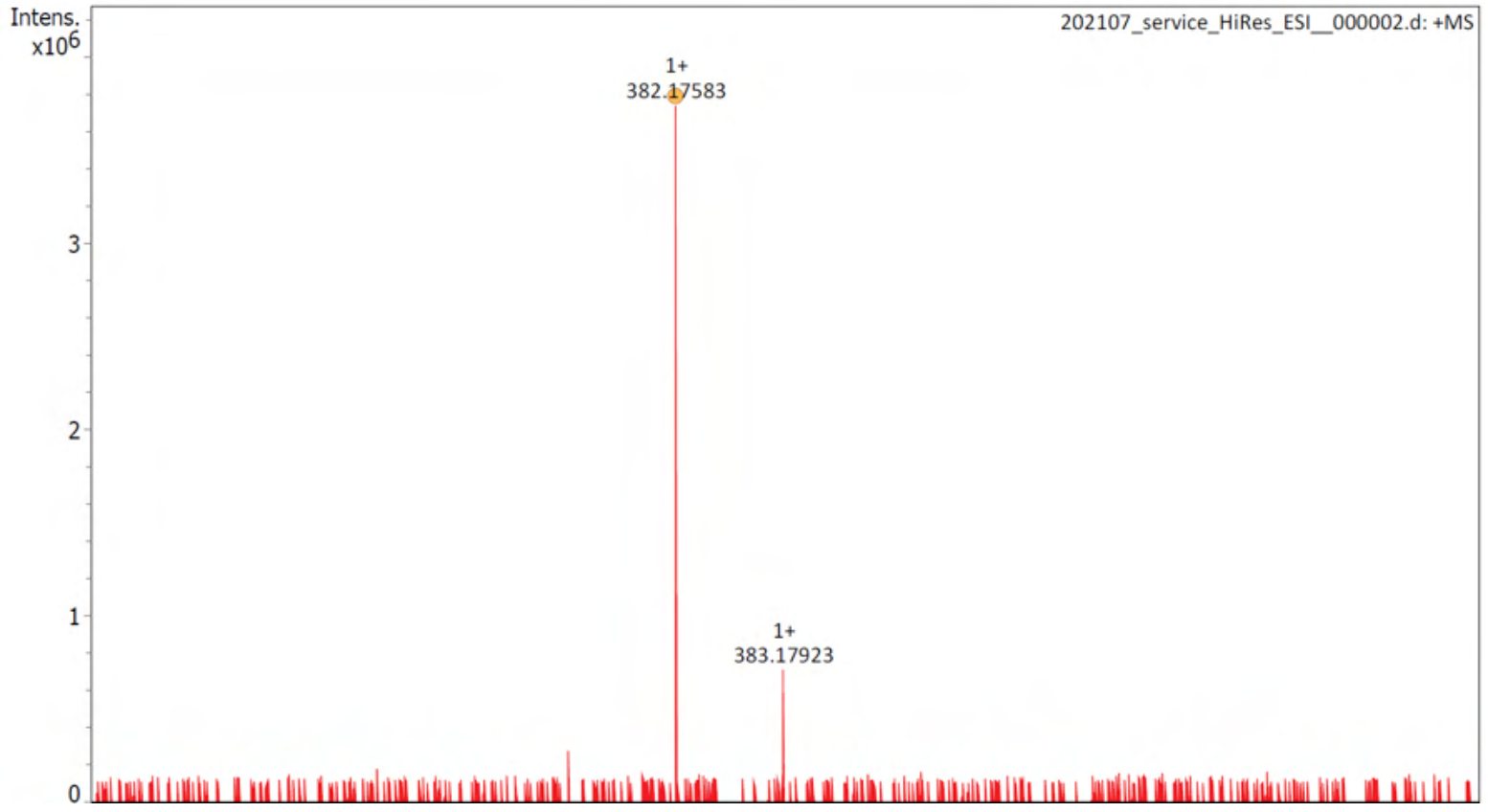

Figure S15. HR-MS (ESI+) spectrum of $N$-(adamantylmethyl)- $N^{\prime}$-(4-nitrophenyl)squaramide. 
1-(1-adamantyl)-3-(4-nitrophenyl)urea (4)<smiles>O=C(Nc1ccc([N+](=O)[O-])cc1)NC12CC3CC(CC(C3)C1)C2</smiles>
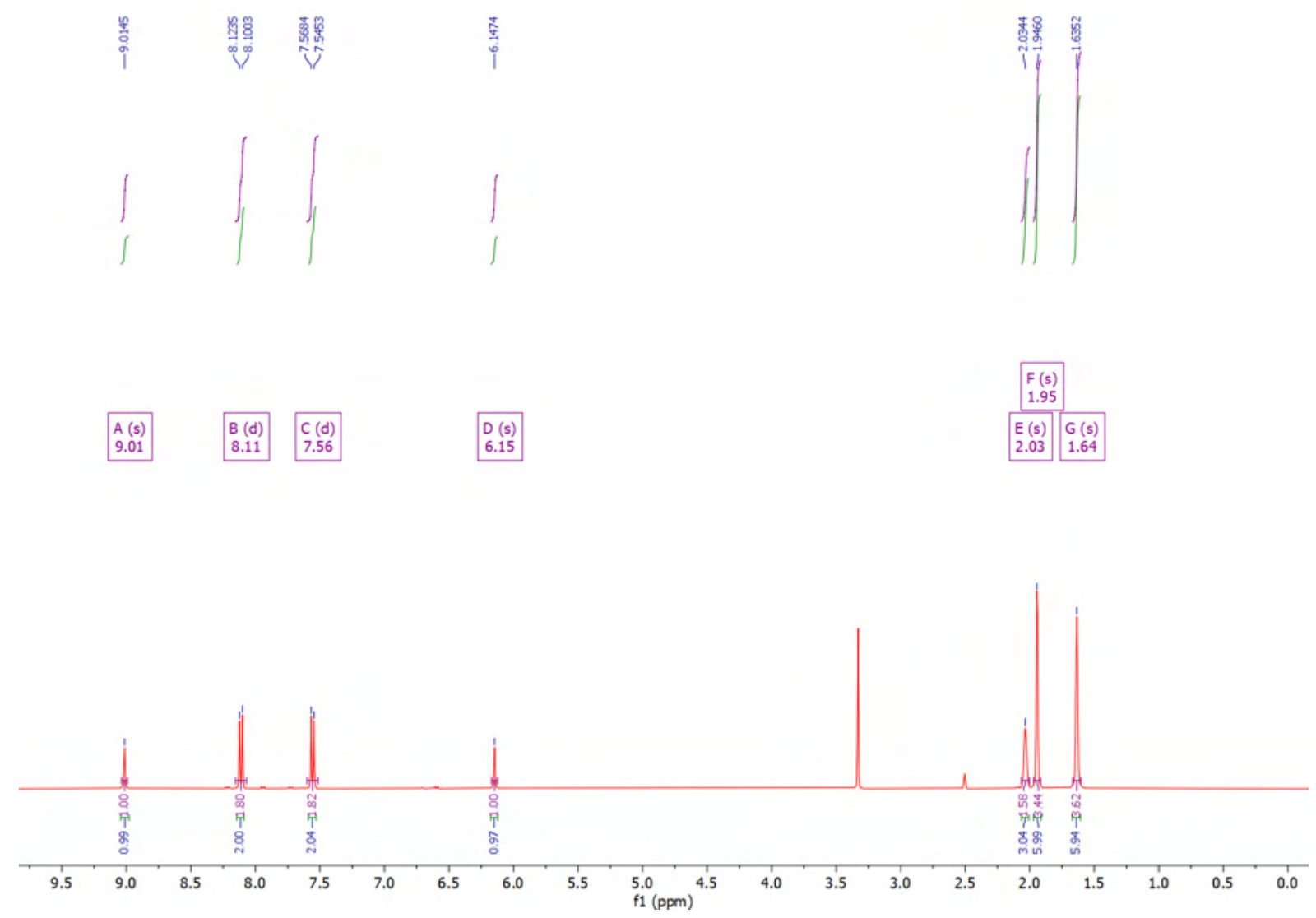

Figure S16. ${ }^{1} \mathrm{H}$ NMR (500 MHz) spectrum of 1-adamantyl-3-(4-nitrophenyl)urea in DMSO-d6 at $298 \mathrm{~K}$. 


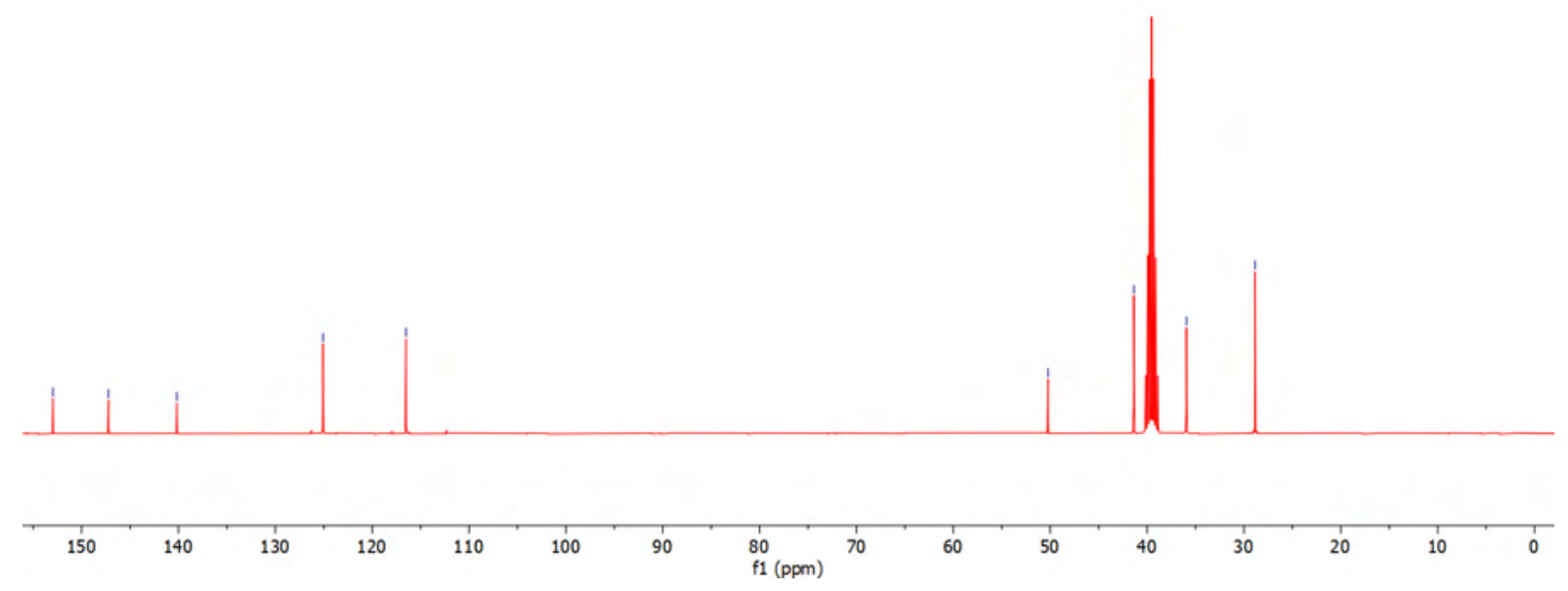

Figure S17. ${ }^{13} \mathrm{C}$ NMR (101 MHz) spectrum of 1-adamantyl-3-(4-nitrophenyl)urea in DMSO-d6 at $298 \mathrm{~K}$.

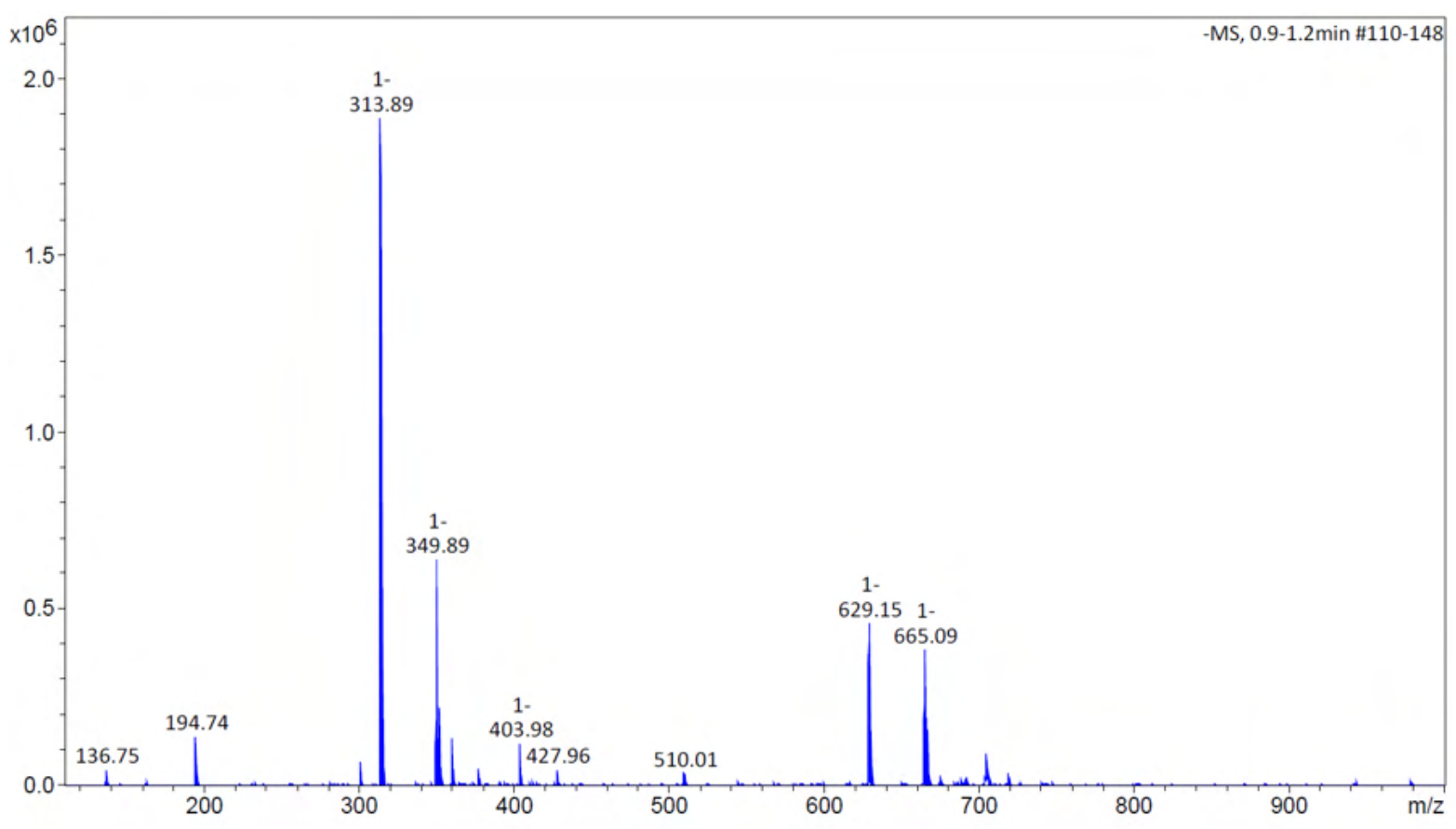

Figure S18. LR-MS (ESI-) spectrum of 1-adamantyl-3-(4-nitrophenyl)urea. 


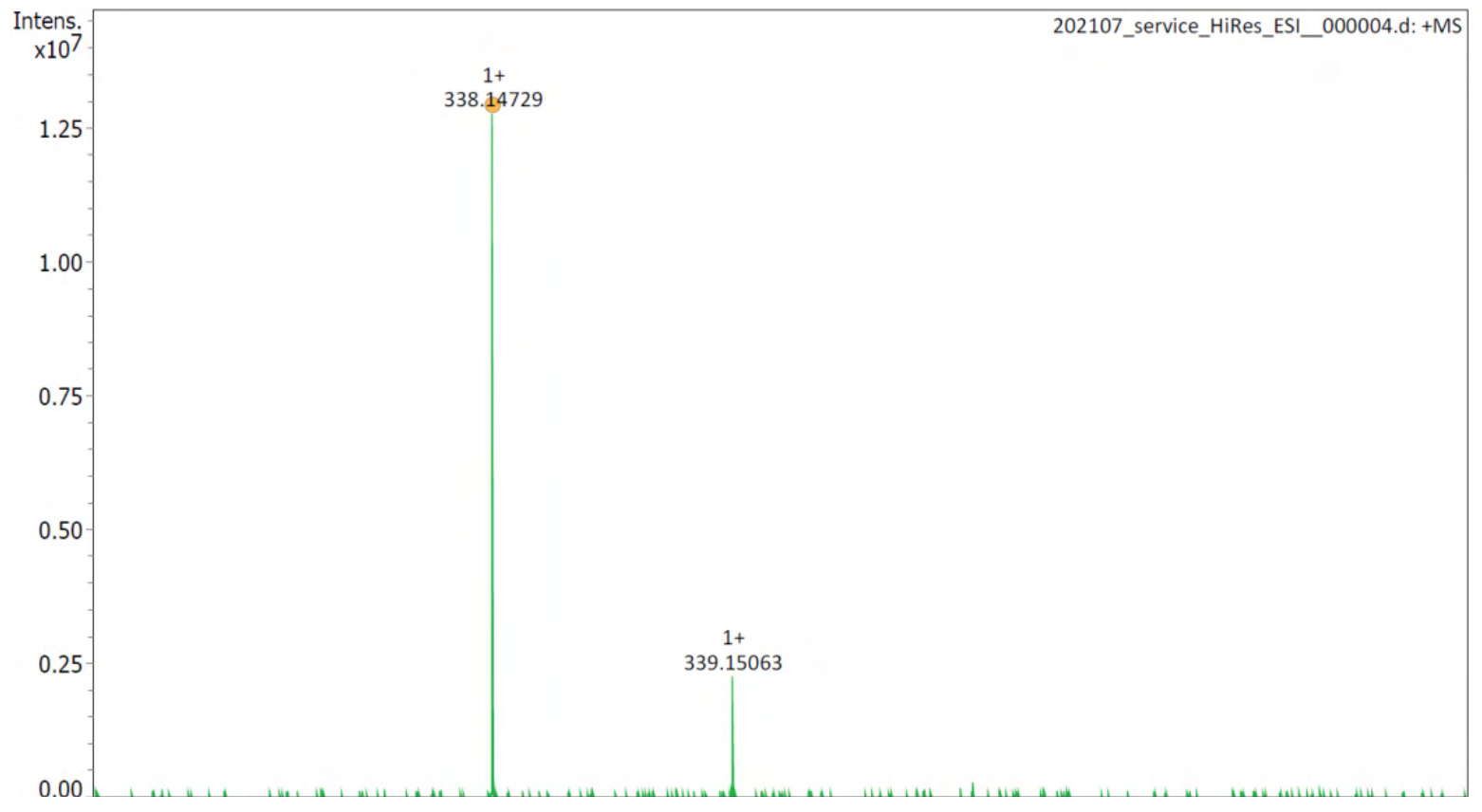

Figure S19. HR-MS (ESI+) spectrum of 1-adamantyl-3-(4-nitrophenyl)urea. 
1-adamantyl-3-(4-nitrophenyl)thiourea (5)<smiles>O=[N+]([O-])c1ccc(NC(=S)NC23CC4CC(CC(C4)C2)C3)cc1</smiles>

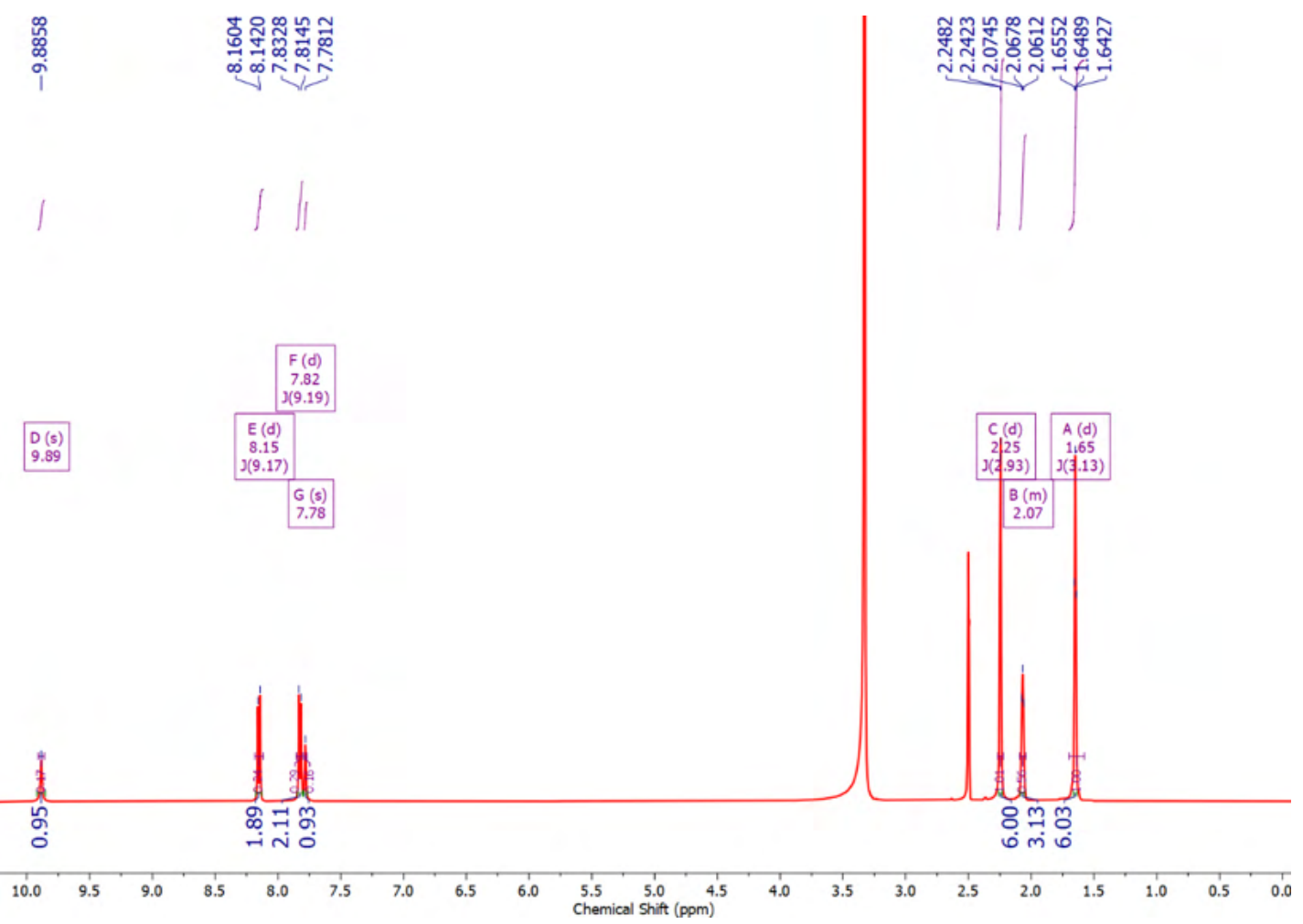

Figure S20. ${ }^{1} \mathrm{H}$ NMR (500 MHz) spectrum of 1-adamantyl-3-(4-nitrophenyl)thiourea in DMSO- $d_{6}$ at $298 \mathrm{~K}$. 


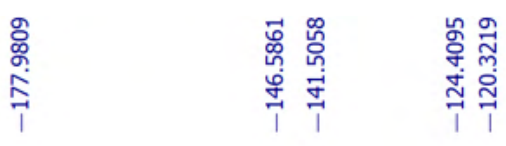
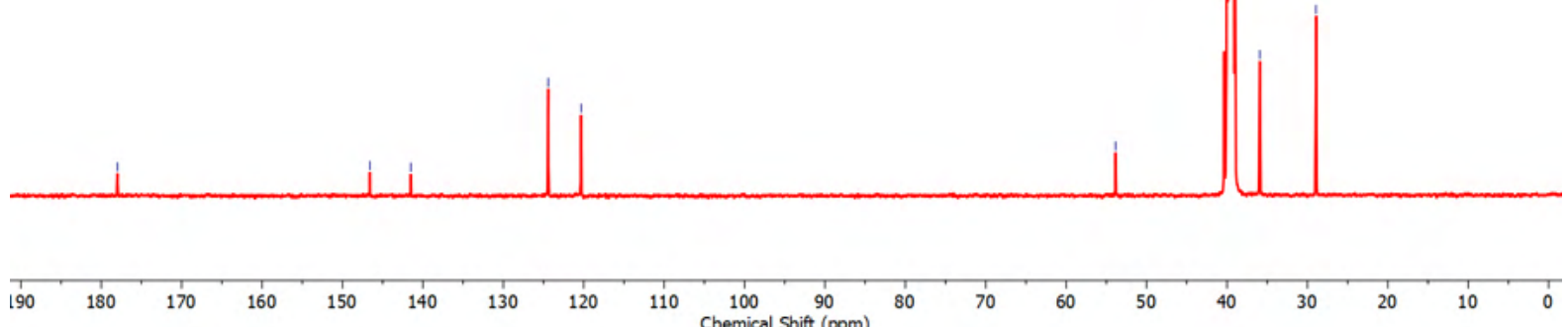

Figure S21. ${ }^{13} \mathrm{C}$ NMR (101 MHz) spectrum of 1-adamantyl-3-(4-nitrophenyl)thiourea in DMSO- $d_{6}$ at $298 \mathrm{~K}$.

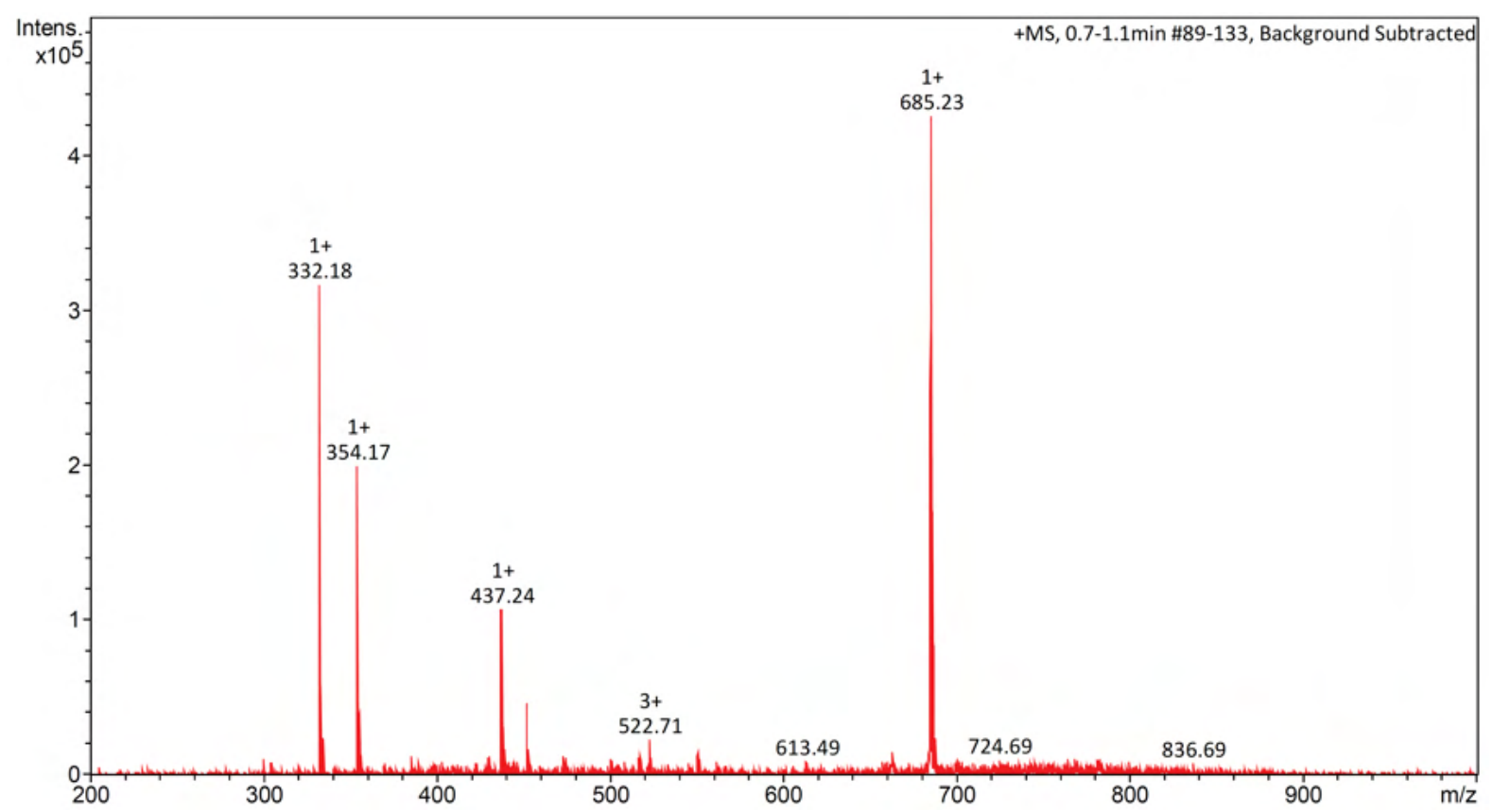

Figure S22. LR-MS (ESI+) spectrum of 1-adamantyl-3-(4-nitrophenyl)thiourea. 


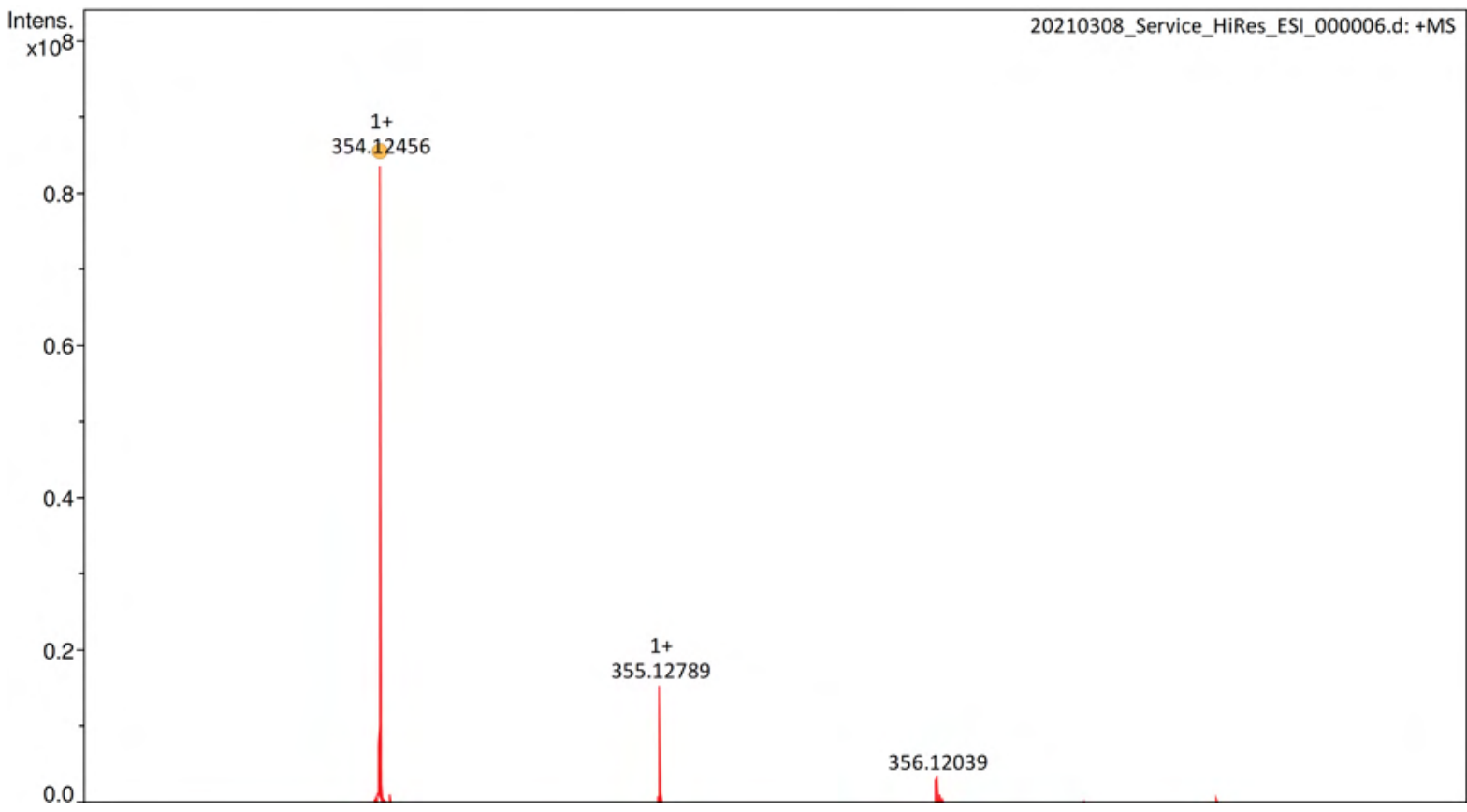

Figure S23. HR-MS (ESI+) spectrum of 1-adamantyl-3-(4-nitrophenyl)thiourea. 
$N$-(adamantyl)- $N^{\prime}$-(4-nitrophenyl)squaramide (6)
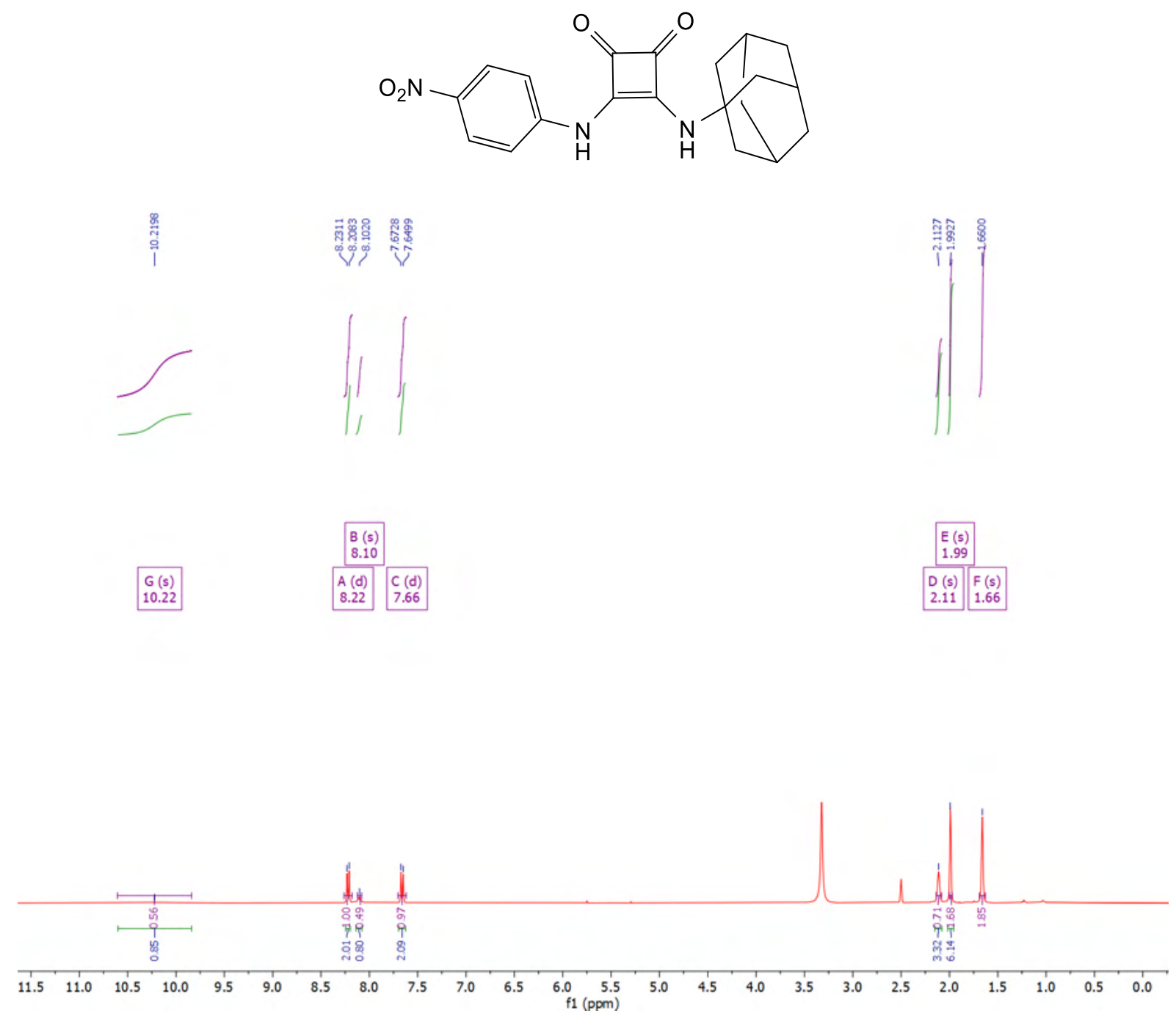

Figure S24. ${ }^{1} \mathrm{H}$ NMR (500 MHz) spectrum of $N$-(adamantyl)- $N$ '-(4-nitrophenyl)squaramide in DMSO- $d_{6}$ at $298 \mathrm{~K}$. 


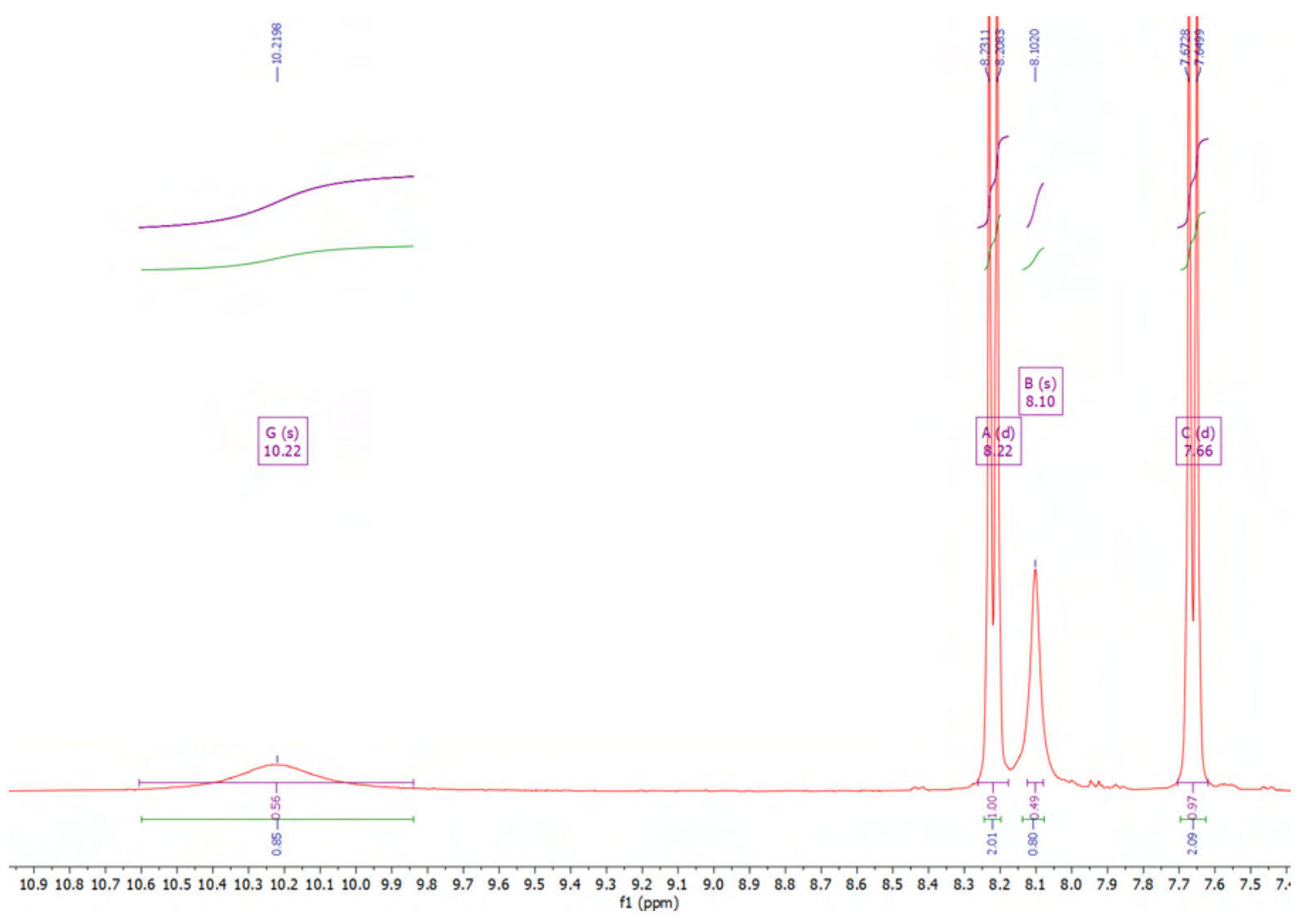

Figure S25. ${ }^{1} \mathrm{H}$ NMR (500 MHz) spectrum of $N$-(adamantyl)- $N$-(4-nitrophenyl)squaramide in DMSO- $d_{6}$ at $298 \mathrm{~K}$. Zoomed in to display broad resonance attributed to the squaramide $\mathrm{N}-\mathrm{H}$ proton. 


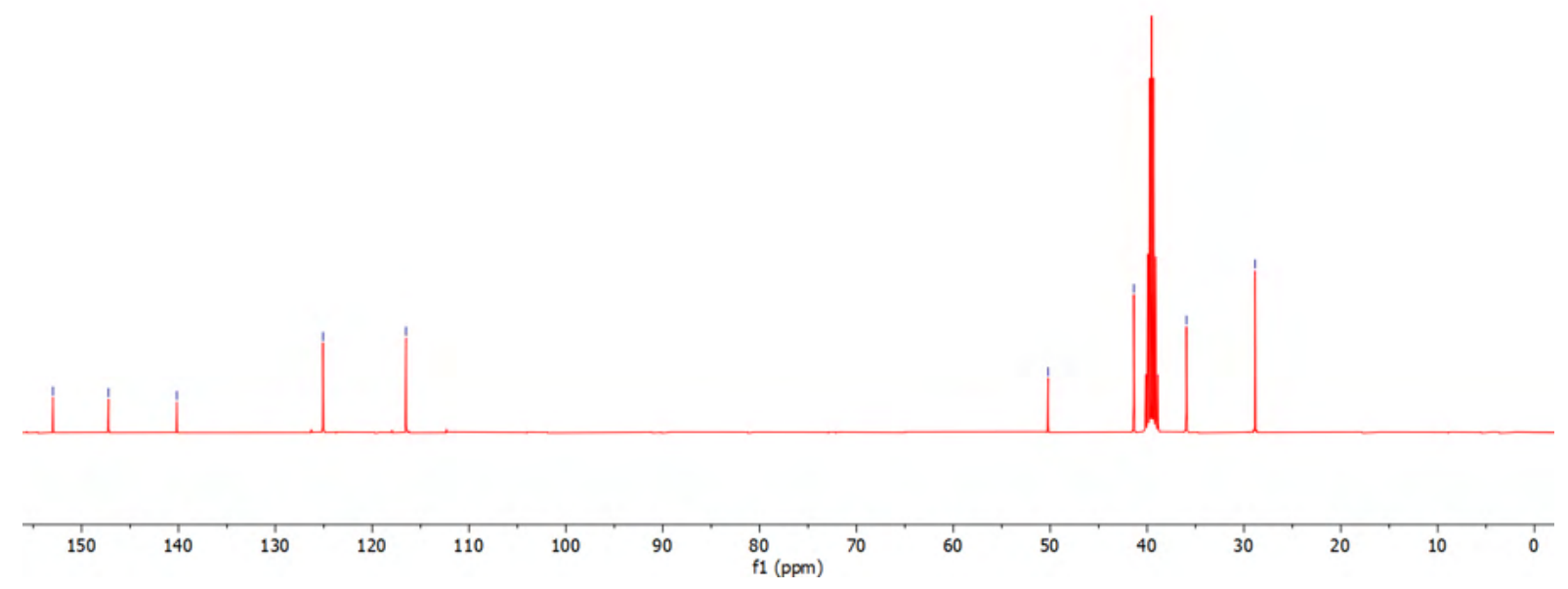

Figure S26. ${ }^{13} \mathrm{C}$ NMR (101 MHz) spectrum of $N$-(adamantyl)- $N$ '-(4-nitrophenyl)squaramide urea in DMSO- $d_{6}$ at $298 \mathrm{~K}$.

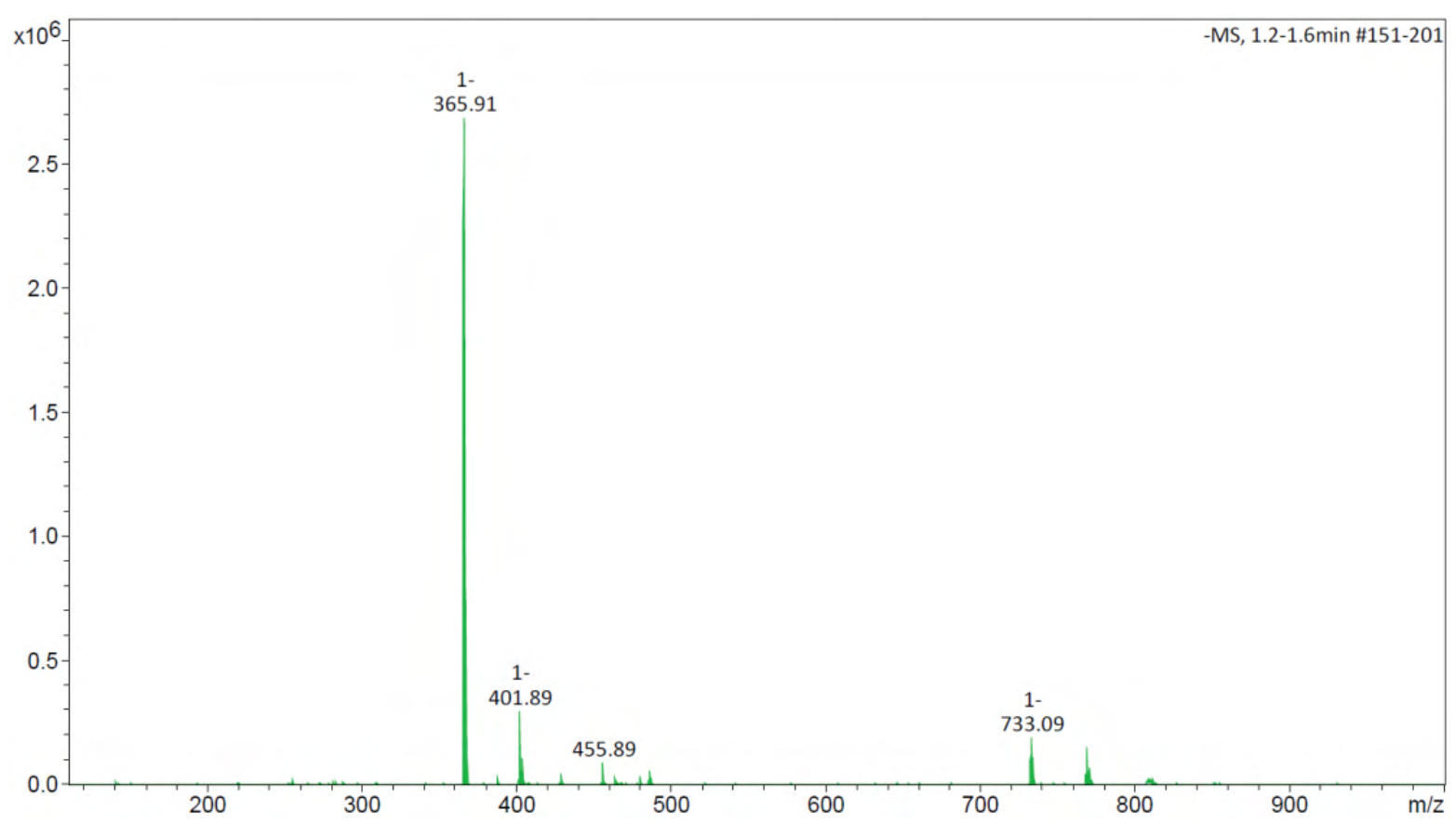

Figure S27. LR-MS (ESI-) spectrum of $N$-(adamantyl)- $N$ '-(4-nitrophenyl)squaramide. 


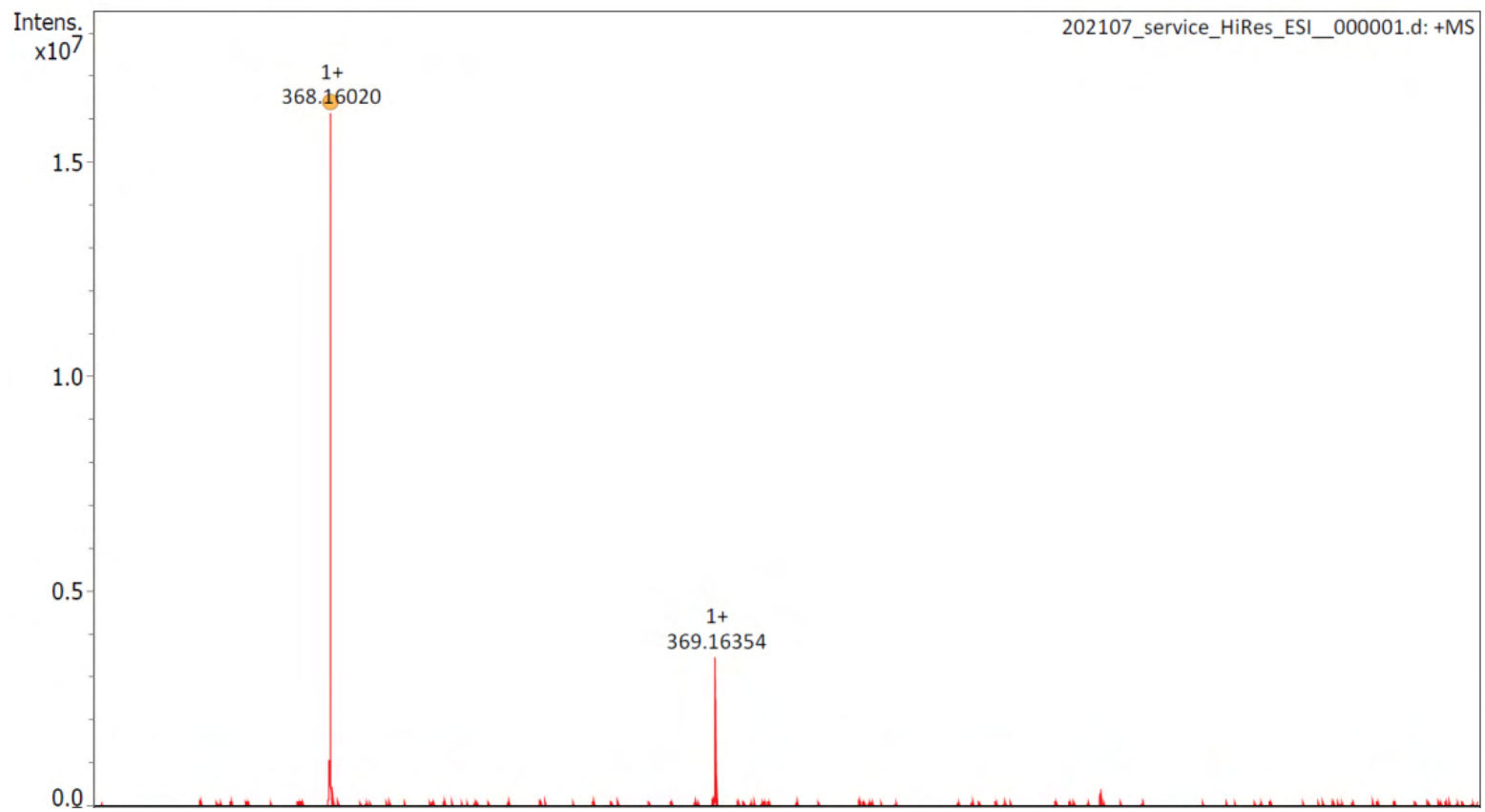

Figure S28. HR-MS (ESI $\left.{ }^{+}\right)$spectrum of $N$-(adamantyl)- $N^{\prime}$-(4-nitrophenyl)squaramide. 


\section{Single Crystal X-ray Diffraction}

Crystals of free receptors 1 and 3 suitable for single crystal X-ray diffraction were obtained by vapour diffusion of $\mathrm{H}_{2} \mathrm{O}$ into a DMSO solution saturated with either compound. The crystals were analysed using singe X-ray crystal diffraction, performed on an Agilent dual-beam Supernova ${ }^{\mathrm{TM}}$ System with a CCD Atlas detector on Mo/ka. Each specimen was mounted with the dual purpose cyro-protective adhesive Paratone- $\mathrm{N}$ and cool to $150 \mathrm{~K}$ with Oxford Cyrosystema Cobra Cooler. Compound 1 crystallised in the monocline space group $P 21 / c$ with one formula unit contained in the asymmetric unit (CDCC 2109212).

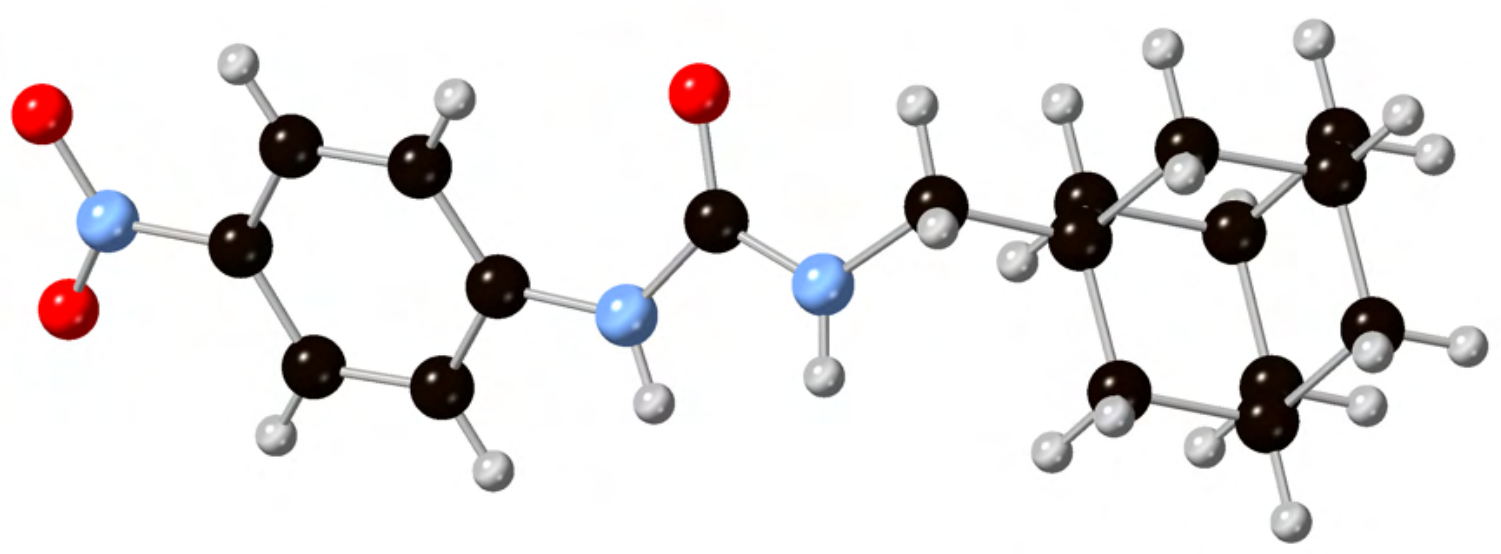

Figure S29. The X-ray crystal structure of receptor 1 (CDCC 2109212). 
Table S1. Crystal data and structure refinement for $\mathbf{1 .}$

\begin{tabular}{|c|c|}
\hline Identification code & $21 \mathrm{dm} 001$ \\
\hline Empirical formula & $\mathrm{C}_{18} \mathrm{H}_{23} \mathrm{~N}_{3} \mathrm{O}_{3}$ \\
\hline Formula weight & 329.39 \\
\hline Temperature/K & $150.00(18)$ \\
\hline Crystal system & monoclinic \\
\hline Space group & $\mathrm{P} 21 / \mathrm{c}$ \\
\hline $\mathrm{a} / \AA$ & $13.6962(3)$ \\
\hline $\mathrm{b} / \AA$ & $13.6338(2)$ \\
\hline$c / \AA$ & $9.2710(2)$ \\
\hline$\alpha /^{\circ}$ & 90 \\
\hline$\beta / /^{\circ}$ & 104.983(3) \\
\hline$\gamma / /^{\circ}$ & 90 \\
\hline Volume/ $/ \AA^{3}$ & $1672.32(7)$ \\
\hline $\mathrm{Z}$ & 4 \\
\hline Qcalcg/ $\mathrm{cm}^{3}$ & 1.308 \\
\hline$\mu / \mathrm{mm}^{-1}$ & 0.090 \\
\hline$F(000)$ & 704.0 \\
\hline Crystal size/mm³ & $0.563 \times 0.328 \times 0.243$ \\
\hline Radiation & $\operatorname{Mo} \mathrm{K} \alpha(\lambda=0.71073)$ \\
\hline \multicolumn{2}{|c|}{$2 \Theta$ range for data collection/ ${ }^{\circ} 5.442$ to 56.56} \\
\hline Index ranges & $-18 \leq \mathrm{h} \leq 18,-18 \leq \mathrm{k} \leq 18,-12 \leq 1 \leq 12$ \\
\hline Reflections collected & 26702 \\
\hline Independent reflections & $4155\left[R_{\text {int }}=0.0255, R_{\text {sigma }}=0.0166\right]$ \\
\hline Data/restraints/parameters & $4155 / 54 / 217$ \\
\hline Goodness-of-fit on $F^{2}$ & 1.042 \\
\hline Final $R$ indexes $[\mathrm{I}>=2 \sigma(\mathrm{I})]$ & $\mathrm{R}_{1}=0.0560, \mathrm{w} \mathrm{R}_{2}=0.1415$ \\
\hline Final $R$ indexes [all data] & $\mathrm{R}_{1}=0.0675, \mathrm{wR}_{2}=0.1521$ \\
\hline
\end{tabular}


Largest diff. peak/hole / e $\AA^{-3} 0.50 /-0.44$

Table S2. Fractional Atomic Coordinates $\left(\times 10^{4}\right)$ and Equivalent Isotropic Displacement Parameters $\left(\AA^{2} \times 10^{3}\right)$ for 1 . $U_{\text {eq }}$ is defined as $1 / 3$ of the trace of the orthogonalised $U_{\text {IJ }}$ tensor.

\begin{tabular}{l|rrrr} 
Atom & \multicolumn{1}{l}{$\boldsymbol{x}$} & \multicolumn{1}{r}{$\boldsymbol{y}$} & $\boldsymbol{U}(\mathrm{eq})$ \\
\hline C1 & $4472.1(12)$ & $4070.3(12)$ & $3587(2)$ & $34.5(4)$ \\
C2 & $4839.7(13)$ & $4760.9(13)$ & $2775(2)$ & $39.7(4)$ \\
C3 & $4315.2(13)$ & $5621.5(13)$ & $2377(2)$ & $35.7(4)$ \\
C4 & $3415.8(11)$ & $5795.1(11)$ & $2792.5(16)$ & $25.5(3)$ \\
C5 & $3040.9(12)$ & $5072.7(12)$ & $3575.3(18)$ & $30.0(3)$ \\
C6 & $3575.4(13)$ & $4211.5(12)$ & $3979.6(19)$ & $34.1(4)$ \\
C7 & $2170.5(11)$ & $7108.1(10)$ & $2881.7(16)$ & $23.3(3)$ \\
C8 & $995.6(12)$ & $8496.8(11)$ & $2485.6(19)$ & $29.8(3)$ \\
C9 & $1314.1(11)$ & $9569.6(10)$ & $2736.9(16)$ & $24.2(3)$ \\
C10 & $1464.7(12)$ & $10037.5(11)$ & $1315.9(17)$ & $27.2(3)$ \\
C11 & $2281.3(18)$ & $9675.1(13)$ & $3968(2)$ & $51.3(6)$ \\
C12 & $449.3(17)$ & $10116.9(14)$ & $3171(3)$ & $47.9(5)$ \\
C13 & $1721.1(13)$ & $11126.2(12)$ & $1554(2)$ & $34.0(4)$ \\
C14 & $2695.7(16)$ & $11223.7(14)$ & $2776(3)$ & $64.3(7)$ \\
C15 & $2555(2)$ & $10766.9(16)$ & $4223(3)$ & $76.8(9)$ \\
C16 & $883.5(15)$ & $11663.2(12)$ & $2038(2)$ & $40.2(4)$ \\
C17 & $729(2)$ & $11202.5(14)$ & $3462(3)$ & $56.7(6)$ \\
C18 & $1686(3)$ & $11291.7(17)$ & $4684(3)$ & $86.3(11)$ \\
N1 & $5052.4(12)$ & $3180.0(12)$ & $4068(2)$ & $46.2(4)$ \\
N2 & $2913.8(10)$ & $6671.1(9)$ & $2327.4(15)$ & $27.5(3)$ \\
N3 & $1732.4(10)$ & $7877.5(9)$ & $2054.5(14)$ & $27.0(3)$ \\
O1 & $4722.3(13)$ & $2562.3(11)$ & $4777(2)$ & $63.2(5)$ \\
O2 & $5865.7(11)$ & $3095.9(12)$ & $3751(2)$ & $66.9(5)$ \\
O3 & $1947.5(9)$ & $6815.6(8)$ & $4008.7(12)$ & $31.6(3)$ \\
& & & &
\end{tabular}

Table S3. Hydrogen Atom Coordinates $\left(\AA \times 10^{4}\right)$ and Isotropic Displacement Parameters $\left(\AA^{2} \times 10^{3}\right)$ for 1 .

\begin{tabular}{l|rrrr} 
Atom & \multicolumn{1}{c}{$x$} & \multicolumn{1}{c}{$\boldsymbol{y}$} & \multicolumn{1}{c}{$\boldsymbol{y}$} & $\mathrm{U}(\mathrm{eq})$ \\
\hline H2 & 5448.98 & 4643.44 & 2493.49 & 48 \\
H3 & 4563.29 & 6100.8 & 1817.6 & 43 \\
H5 & 2419.4 & 5173.02 & 3829.61 & 36 \\
H6 & 3328.24 & 3721.41 & 4522.6 & 41 \\
H8A & 868.93 & 8234.12 & 3416.19 & 36
\end{tabular}




\begin{tabular}{l|rrrr} 
Atom & \multicolumn{1}{c}{$x$} & \multicolumn{1}{c}{$\boldsymbol{y}$} & $\boldsymbol{U}(\mathrm{eq})$ \\
\hline H8B & 351.73 & 8463.68 & 1697.87 & 36 \\
H10A & 839.85 & 9961.77 & 500.51 & 33 \\
H10B & 2018.74 & 9696.57 & 1013.89 & 33 \\
H11A & 2836.74 & 9322.64 & 3686.75 & 62 \\
H11B & 2190.79 & 9381.52 & 4901.34 & 62 \\
H12A & -177.8 & 10060.63 & 2354.49 & 57 \\
H12B & 324.5 & 9818.23 & 4079.79 & 57 \\
H13 & 1809.71 & 11419.78 & 607.54 & 41 \\
H14A & 2877.89 & 11924.85 & 2942.96 & 77 \\
H14B & 3250.62 & 10885.48 & 2472.55 & 77 \\
H15 & 3193.6 & 10834.9 & 5034.83 & 92 \\
H16A & 247.49 & 11624.83 & 1235.02 & 48 \\
H16B & 1065.77 & 12363.51 & 2217.19 & 48 \\
H17 & 170.38 & 11551.18 & 3762.83 & 68 \\
H18A & 1590.19 & 10994.81 & 5612.51 & 104 \\
H18B & 1857.44 & 11992.8 & 4882.1 & 104 \\
H2A & 3086.64 & 6983.58 & 1600.37 & 33 \\
H3A & 1899.7 & 8009.58 & 1220.5 & 32
\end{tabular}

Crystals of compound $\mathbf{1}$ suitable for single crystal X-ray diffraction were also obtained in the presence of $\mathrm{TBACl}$ (20 molar equiv.). Crystals were prepared by slow evaporation of a saturated DMSO solution containing 1 and $\mathrm{TBACl}$. The collection was carried out in the same manner as the parent molecule 1. 1.TBACl crystallised in the centrosymmetric triclinic $\mathrm{P} \overline{1}$ space group (CDCC 2109213). Receptor 1 was found to form a 1:1 receptor:anion complex with $\mathrm{TBACl}$, with coordinating hydrogen bonding interactions existing between chloride and the protons of the urea moiety $(\mathrm{N} \cdots \mathrm{Cl}$ distances: $3.145-3.278 \AA, \mathrm{N}-\mathrm{H} \cdots \mathrm{Cl}$ angles: 150.5(7)-165.6(7) ${ }^{\circ}$ ) within the unit cell. The remaining bonds primarily extend outside the asymmetric unit, with large coordination created between the carbon donated hydrogen atoms an external chloride ion. 


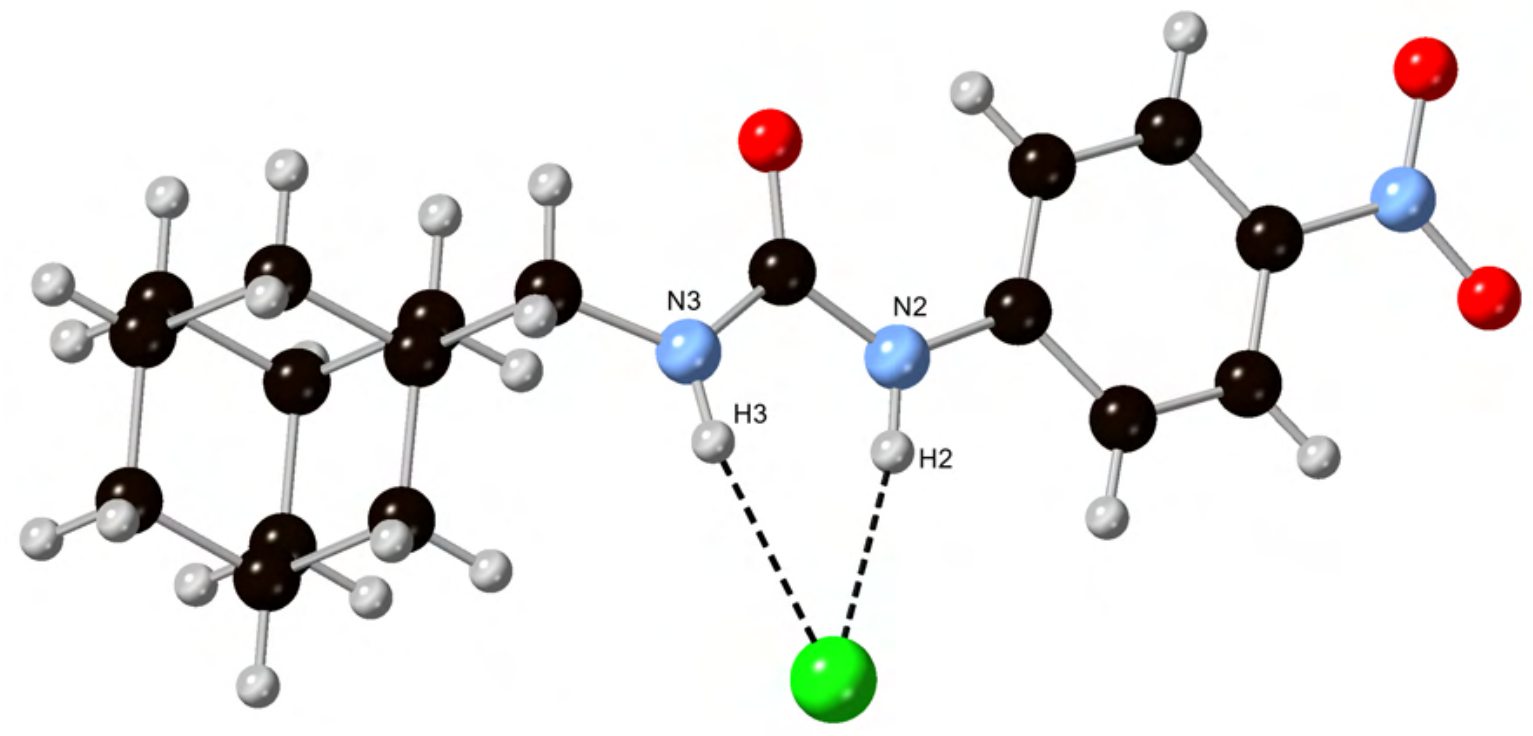

Figure S30. The X-ray crystal structure of receptor $\mathbf{1}$ in the presence of TBACl (CDCC 2109213). The chloride anion is coloured green, and the counterion is omitted for clarity. Dashed lines represent hydrogen bonds.

Table S4. Crystal data and structure refinement for 1+TBACl.

Identification code

Empirical formula

Formula weight

Temperature/K

Crystal system

Space group
$\mathrm{a} / \AA$
8.1181(2)
b/Å
c/Å
$\alpha{ }^{\circ}$
$\beta /{ }^{\circ}$
$\gamma /{ }^{\circ}$
Volume $/ \AA^{3}$
Z
13.6649(4)
17.6062(6)
108.557(3)
100.746(2)
95.887(2)
1791.40(10)
2

21bh013

$\mathrm{C}_{34} \mathrm{H}_{59} \mathrm{ClN}_{4} \mathrm{O}_{3}$

607.30

150.0(2)

triclinic

P-1 


\begin{tabular}{|c|c|}
\hline Qcalcg/ $\mathrm{cm}^{3}$ & 1.126 \\
\hline$\mu / \mathrm{mm}^{-1}$ & 0.143 \\
\hline$F(000)$ & 664.0 \\
\hline Crystal size $/ \mathrm{mm}^{3}$ & $0.91 \times 0.74 \times 0.53$ \\
\hline Radiation & Mo K $\alpha(\lambda=0.71073)$ \\
\hline \multicolumn{2}{|c|}{$2 \Theta$ range for data collection $/{ }^{\circ} 5.018$ to 52.742} \\
\hline Index ranges & $-10 \leq \mathrm{h} \leq 10,-17 \leq \mathrm{k} \leq 17,-21 \leq 1 \leq 21$ \\
\hline Reflections collected & 25252 \\
\hline Independent reflections & $7304\left[R_{\text {int }}=0.0292, R_{\text {sigma }}=0.0291\right]$ \\
\hline Data/restraints/parameters & $7304 / 0 / 383$ \\
\hline Goodness-of-fit on $F^{2}$ & 1.034 \\
\hline Final $R$ indexes $[\mathrm{I}>=2 \sigma(\mathrm{I})]$ & $\mathrm{R}_{1}=0.0422, \mathrm{wR}_{2}=0.1050$ \\
\hline Final $R$ indexes [all data] & $\mathrm{R}_{1}=0.0562, \mathrm{wR}_{2}=0.1144$ \\
\hline Largest diff. peak/hole / e $\AA^{-3}$ & $0.75 /-0.28$ \\
\hline
\end{tabular}

Table S5. Fractional Atomic Coordinates $\left(\times 10^{4}\right)$ and Equivalent Isotropic Displacement Parameters $\left(\AA^{2 \times 10^{3}}\right)$ for $1+\mathrm{TBACl}$. U Ueq is defined as $1 / 3$ of the trace of the orthogonalised UIJ tensor.

\begin{tabular}{|c|c|c|c|c|}
\hline Atom & $x$ & $y$ & $z$ & $U(e q)$ \\
\hline $\mathrm{Cl}$ & 1093.0(19) & $7160.4(12)$ & $5471.9(9)$ & $28.4(3)$ \\
\hline $\mathrm{Cl}^{\prime}$ & $4340.7(18)$ & $5118.9(12)$ & $2541.1(9)$ & $25.6(3)$ \\
\hline $\mathrm{C}^{\prime}$ & 4638(2) & $4874.0(13)$ & $3334.4(10)$ & $31.5(4)$ \\
\hline $\mathrm{C} 2$ & $1196.8(19)$ & $6429.6(12)$ & $4733.5(10)$ & $29.4(3)$ \\
\hline $\mathrm{C} 3$ & $1093.6(19)$ & $6728.7(12)$ & 4053.2(9) & $27.0(3)$ \\
\hline $\mathrm{C} 4$ & $886.4(18)$ & $7752.4(12)$ & $4096.8(9)$ & $24.8(3)$ \\
\hline C4' & $3100(2)$ & 4137.2(13) & $3341.0(10)$ & $30.8(4)$ \\
\hline C5 & $800(2)$ & $8474.1(12)$ & $4852.1(10)$ & $30.7(3)$ \\
\hline $\mathrm{C}^{\prime}$ & $3383(2)$ & $3807.7(15)$ & 4097.6(11) & $41.9(4)$ \\
\hline C6 & $892(2)$ & $8172.3(13)$ & $5532.1(10)$ & $32.1(4)$ \\
\hline C6' & 6410.5(19) & $6817.9(12)$ & 3151.1(9) & $28.0(3)$ \\
\hline C7 & $468(2)$ & 8899.2(12) & $3244.4(0)$ & $28.3(3)$ \\
\hline$C 7^{\prime}$ & $5081(2)$ & 7516.(13) & $3305.4(10)$ & $36.4(4)$ \\
\hline C8 & $2592)$ & 9711.5(12) & 2180.1(10) & $30.7(3)$ \\
\hline
\end{tabular}




\begin{tabular}{|c|c|c|c|c|}
\hline Atom & $x$ & $y$ & $z$ & $U(e q)$ \\
\hline \begin{tabular}{l|l} 
C $8^{\prime}$ \\
\end{tabular} & $5823(3)$ & $8523.6(15)$ & $4048.0(11)$ & $48.6(5)$ \\
\hline C9 & 1911(2) & $10062.7(12)$ & 1970.4(9) & $26.0(3)$ \\
\hline C9' & $6062(3)$ & $8360.4(19)$ & $4859.3(12)$ & $61.8(6)$ \\
\hline C10' & $7362.6(18)$ & $5238.9(12)$ & 2376.1(9) & $26.1(3)$ \\
\hline C10 & $3433(2)$ & 10334.2(14) & $2703.2(10)$ & $36.8(4)$ \\
\hline C11' & $7117(2)$ & 4211.6(14) & $1671.9(11)$ & $36.0(4)$ \\
\hline C11 & $1737(2)$ & 11049.6(13) & $1742.8(10)$ & $33.2(4)$ \\
\hline C12' & $8789(2)$ & $3795.0(14)$ & $1685.1(10)$ & $34.8(4)$ \\
\hline C12 & $2247(2)$ & 9208.0(13) & $1231.2(10)$ & $35.2(4)$ \\
\hline C13 & $3365(2)$ & $11436.4(14)$ & $1526.1(12)$ & $41.6(4)$ \\
\hline C13' & $8664(3)$ & $2801.8(17)$ & $962.7(14)$ & $57.7(6)$ \\
\hline C14 & $5066(2)$ & $10707.7(16)$ & $2480.9(13)$ & $48.7(5)$ \\
\hline C14' & $5173.4(18)$ & 5999.2(12) & 1633.5(9) & $25.0(3)$ \\
\hline C15 & 6413(2) & 6701.0(13) & 1410.9(9) & $30.9(4)$ \\
\hline $\mathrm{C} 15^{\prime}$ & $4870(3)$ & $11692.0(15)$ & $2258.8(14)$ & $49.7(5)$ \\
\hline C16 & $5618(2)$ & 6778.6(15) & $581.9(10)$ & $40.6(4)$ \\
\hline C16 & $3871(3)$ & 9588.1(15) & $1010.4(12)$ & $47.1(5)$ \\
\hline C17 & $6823(3)$ & 7414.6(19) & $286.4(13)$ & $60.8(6)$ \\
\hline C17' & $3670(3)$ & $10572.4(15)$ & $786.2(13)$ & $51.1(5)$ \\
\hline C18 & $5368(3)$ & 9851.1(17) & $1743.6(15)$ & $57.1(6)$ \\
\hline N1 & 1156.7(17) & 6849.2(12) & 6190.9(9) & $35.0(3)$ \\
\hline N2 & $5820.0(15)$ & $5789.4(10)$ & 2427.5(7) & $23.1(3)$ \\
\hline N3 & 759.5(16) & $7970.7(10)$ & $3374.5(8)$ & $27.5(3)$ \\
\hline $\mathrm{O} 1$ & 293.2(18) & $8808.6(10)$ & $2445.1(8)$ & $32.0(3)$ \\
\hline $\mathrm{O} 2$ & 936.1(18) & $7480.9(11)$ & $6823.2(8)$ & $46.6(3)$ \\
\hline $\mathrm{O} 3$ & $1413.8(18)$ & $5957.0(10)$ & 6135.2(8) & $49.8(3)$ \\
\hline $\mathrm{Cl1}^{*}$ & $364.0(17)$ & $9689.0(9)$ & $3789.4(7)$ & $41.3(3)$ \\
\hline
\end{tabular}

Table S6. Hydrogen Atom Coordinates $\left(\AA \times 10^{4}\right)$ and Isotropic Displacement Parameters $\left(\AA^{2} \times 10^{3}\right)$ for $1+\mathrm{TBACl}$.

\begin{tabular}{lrrrr} 
Atom & \multicolumn{1}{l}{$\boldsymbol{x}$} & \multicolumn{1}{c}{$\boldsymbol{y}$} & $\boldsymbol{z}$ & $\mathrm{U}(\mathrm{eq})$ \\
\hline H1'A & 4041.96 & 4448 & 2071.47 & 31 \\
H1'B & 3345.16 & 5479.53 & 2516.29 & 31 \\
H2'A & 4838.51 & 5532.59 & 3811.62 & 38 \\
H2'B & 5663.24 & 4542.87 & 3384.07 & 38 \\
H2 & 1336.5 & 5736.1 & 4698.84 & 35 \\
H3 & 1163.58 & 6234.91 & 3544.63 & 32 \\
H4'A & 2095.63 & 4490.75 & 3326.18 & 37
\end{tabular}




\begin{tabular}{|c|c|c|c|c|}
\hline Atom & $x$ & $y$ & $z$ & $U(e q)$ \\
\hline $\mathrm{H} 4$ 'B & 2853.26 & 3504.2 & 2839.62 & 37 \\
\hline H5 & 677.35 & 9172.56 & 4895.55 & 37 \\
\hline H5'A & 4428.66 & 3508.33 & 4139.95 & 63 \\
\hline H5'B & 2414.43 & 3281.95 & 4047.67 & 63 \\
\hline $\mathrm{H}^{\prime} \mathrm{C}$ & 3488.31 & 4420.75 & 4592.07 & 63 \\
\hline H6 & 818.22 & 8659.77 & 6042.58 & 38 \\
\hline H6'A & 6817.06 & 6654.63 & 3655.78 & 34 \\
\hline H6'B & 7393.94 & 7217.42 & 3057.18 & 34 \\
\hline H7' A & 4094.07 & 7132.95 & 3409.24 & 44 \\
\hline H7'B & 4676.62 & 7700.06 & 2810.15 & 44 \\
\hline H8A & 26.18 & 10301.78 & 2623.02 & 37 \\
\hline $\mathrm{H} 8 \mathrm{~B}$ & -689.15 & 9539.22 & 1689.08 & 37 \\
\hline H8'A & 6936.97 & 8824.42 & 3991.41 & 58 \\
\hline H8'B & 5054.88 & 9040.27 & 4041.73 & 58 \\
\hline H9'A & 4964.58 & 8064.29 & 4922.15 & 93 \\
\hline H9'B & 6511.5 & 9033.74 & 5305.06 & 93 \\
\hline $\mathrm{H}^{\prime} \mathrm{C}$ & 6864.98 & 7876.34 & 4882.44 & 93 \\
\hline H10A & 8305.91 & 5724.91 & 2334.69 & 31 \\
\hline H10B & 7725.97 & 5108.08 & 2898.75 & 31 \\
\hline H10C & 3562.77 & 9708.83 & 2862.48 & 44 \\
\hline H10D & 3232.88 & 10892.64 & 3179.16 & 44 \\
\hline $\mathrm{H} 11 \mathrm{~A}$ & 6725.38 & 4318.87 & 1141.66 & 43 \\
\hline H11B & 6235.94 & 3694.48 & 1723.09 & 43 \\
\hline $\mathrm{H} 11 \mathrm{C}$ & 759.6 & 10888.89 & 1266.5 & 40 \\
\hline H11D & 1515.14 & 11607.62 & 2212.54 & 40 \\
\hline $\mathrm{H} 12 \mathrm{~A}$ & 9683.36 & 4339.29 & 1676.86 & 42 \\
\hline $\mathrm{H} 12 \mathrm{~B}$ & 9136.96 & 3652.95 & 2204.03 & 42 \\
\hline $\mathrm{H} 12 \mathrm{C}$ & 2367.19 & 8566.31 & 1368.26 & 42 \\
\hline H12D & 1270.27 & 9032.41 & 752.34 & 42 \\
\hline H13 & 3233.24 & 12079.12 & 1381.03 & 50 \\
\hline $\mathrm{H} 13 \mathrm{~A}$ & 7787.48 & 2257.53 & 970.16 & 87 \\
\hline H13B & 9763.05 & 2561.82 & 1003.49 & 87 \\
\hline $\mathrm{H} 13 \mathrm{C}$ & 8359.07 & 2943.49 & 447.22 & 87 \\
\hline H14 & 6054.4 & 10872.76 & 2961.94 & 58 \\
\hline $\mathrm{H} 14 \mathrm{~A}$ & 4832.34 & 5318.37 & 1174.76 & 30 \\
\hline H14B & 4139.03 & 6318.34 & 1680.38 & 30 \\
\hline H15A & 7476.66 & 6410.61 & 1380.39 & 37 \\
\hline H15B & 6698.22 & 7407.23 & 1839.6 & 37 \\
\hline $\mathrm{Hl} 5 \mathrm{C}$ & 4680.89 & 12254.2 & 2735.58 & 60 \\
\hline H15D & 5922.42 & 11943.94 & 2119.71 & 60 \\
\hline H16A & 5256.38 & 6063.69 & 167.75 & 49 \\
\hline
\end{tabular}


Compound 3 crystallised in the monoclinic space group $C 2 / c$ with one formula unit contained in the asymmetric unit (CDCC 2109211). Close hydrogen bonds exist between the squaramide

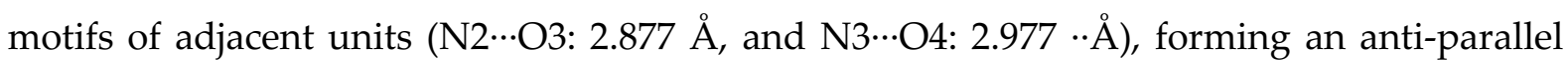
homogenous synthon, this hydrogen bond motif forms along the c-axis, creating sheets of transporters a 242 packing formation (respective to the b-axis).

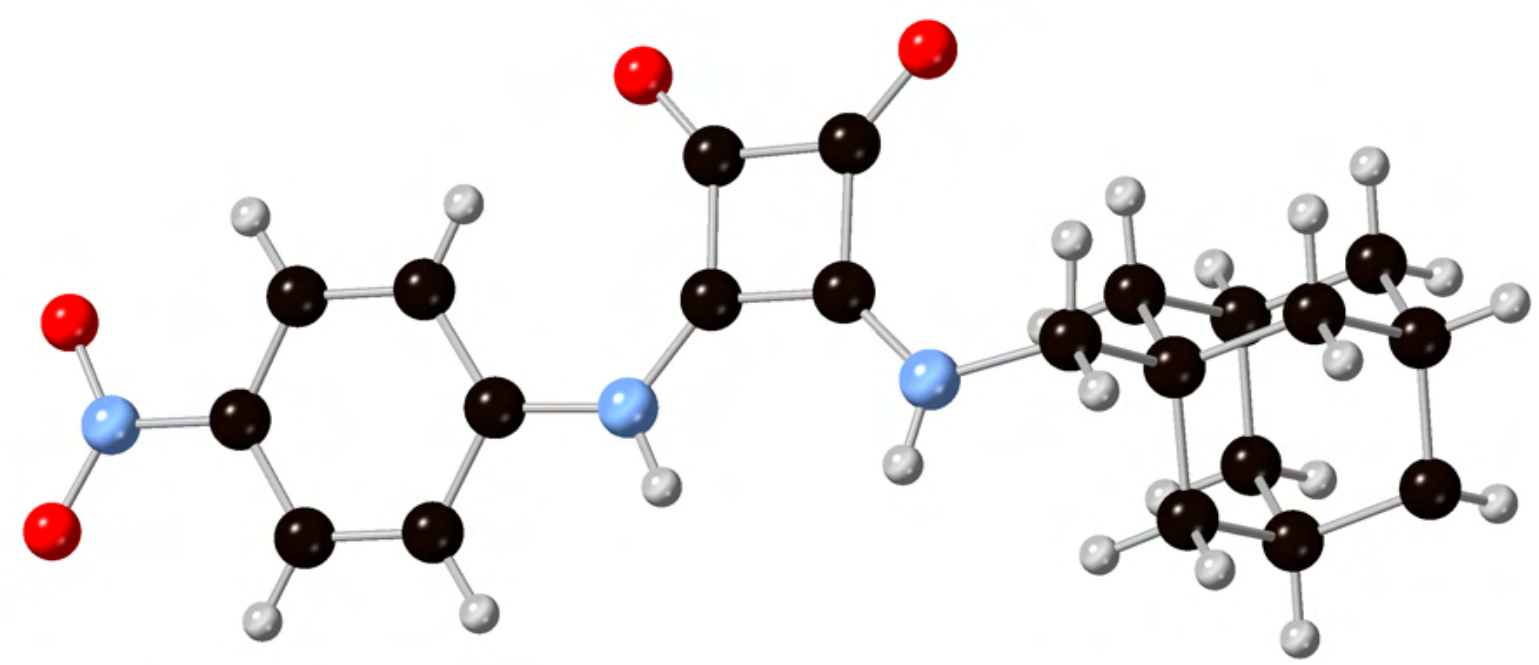

Figure S31. The X-ray crystal structure of receptor 3 (CDCC 2109211).

Table S7. Crystal data and structure refinement for 3.

\begin{tabular}{|c|c|}
\hline Identification code & 21bh014 \\
\hline Empirical formula & $\mathrm{C}_{21} \mathrm{H}_{23} \mathrm{~N}_{3} \mathrm{O}_{4}$ \\
\hline Formula weight & 381.42 \\
\hline Temperature/K & $150.0(2)$ \\
\hline Crystal system & monoclinic \\
\hline Space group & $\mathrm{C} 2 / \mathrm{c}$ \\
\hline $\mathrm{a} / \AA$ & $42.9415(11)$ \\
\hline $\mathrm{b} / \AA$ & $6.2417(2)$ \\
\hline $\mathrm{c} / \AA ̊$ & $13.2277(3)$ \\
\hline$\alpha /^{\circ}$ & 90 \\
\hline$\beta /{ }^{\circ}$ & $94.680(2)$ \\
\hline$\gamma /{ }^{\circ}$ & 90 \\
\hline Volume $/ \AA^{3}$ & $3533.57(17)$ \\
\hline Z & 8 \\
\hline Qcalcg $/ \mathrm{cm}^{3}$ & 1.434 \\
\hline$\mu / \mathrm{mm}^{-1}$ & 0.101 \\
\hline $\mathrm{F}(000)$ & 1616.0 \\
\hline Crystal size/mm³ & $0.374 \times 0.325 \times 0.168$ \\
\hline
\end{tabular}


Radiation

$\operatorname{Mo} \mathrm{K} \alpha(\lambda=0.71073)$

$2 \Theta$ range for data collection/ $/{ }^{\circ} 5.712$ to 52.742

Index ranges

$-53 \leq \mathrm{h} \leq 53,-7 \leq \mathrm{k} \leq 7,-16 \leq 1 \leq 16$

Reflections collected

25030

Independent reflections

$3615\left[R_{\text {int }}=0.0344, R_{\text {sigma }}=0.0204\right]$

Data/restraints/parameters

$3615 / 0 / 253$

Goodness-of-fit on $\mathrm{F}^{2}$

1.029

Final $R$ indexes $[\mathrm{I}>=2 \sigma(\mathrm{I})] \quad \mathrm{R}_{1}=0.0397, \mathrm{wR}_{2}=0.0943$

Final $\mathrm{R}$ indexes [all data] $\quad \mathrm{R}_{1}=0.0471, \mathrm{wR}_{2}=0.0990$

Largest diff. peak/hole / e $\AA^{-3} 0.30 /-0.17$

Table S8. Fractional Atomic Coordinates $\left(\times 10^{4}\right)$ and Equivalent Isotropic Displacement Parameters $\left(\AA^{2} \times 10^{3}\right)$ for 3 . $U_{\text {eq }}$ is defined as $1 / 3$ of of the trace of the orthogonalised $U_{\text {IJ }}$ tensor.

\begin{tabular}{|c|c|c|c|c|}
\hline Atom & $x$ & $y$ & $z$ & $U(e q)$ \\
\hline $\mathrm{C} 21$ & $5499.6(3)$ & $2949(2)$ & $4874.3(11)$ & $21.9(3)$ \\
\hline $\mathrm{C} 1$ & 8029.1(3) & $3413(2)$ & $6886.8(10)$ & $20.5(3)$ \\
\hline $\mathrm{C} 2$ & $7867.4(3)$ & $5158(2)$ & $6473.0(10)$ & $20.7(3)$ \\
\hline C3 & $7560.2(3)$ & $4918(2)$ & $6089.5(10)$ & $19.6(3)$ \\
\hline $\mathrm{C} 4$ & $7413.3(3)$ & $2933(2)$ & 6151.0(9) & $16.2(3)$ \\
\hline C5 & $7584.0(3)$ & $1165(2)$ & $6545.4(10)$ & $18.7(3)$ \\
\hline C6 & $7892.9(3)$ & $1404(2)$ & $6910.6(10)$ & $20.9(3)$ \\
\hline C7 & $6884.9(3)$ & $4122(2)$ & $5511.4(9)$ & $16.6(3)$ \\
\hline $\mathrm{C} 8$ & $6857.9(3)$ & $6463(2)$ & $5479.9(10)$ & $17.8(3)$ \\
\hline C9 & $6535.6(3)$ & $6228(2)$ & $4986.5(10)$ & $19.4(3)$ \\
\hline C10 & $6571.7(3)$ & $3863(2)$ & $5088.7(10)$ & $17.8(3)$ \\
\hline C11 & $6054.5(3)$ & $2532(2)$ & $4514.0(11)$ & $21.2(3)$ \\
\hline $\mathrm{C} 12$ & $5831.3(3)$ & 2491(2) & 5361.1(10) & $18.5(3)$ \\
\hline C13 & $5919.0(3)$ & $4214(2)$ & $6166.2(10)$ & $20.2(3)$ \\
\hline C14 & $5683.2(3)$ & $4221(2)$ & 6973.7(11) & $24.2(3)$ \\
\hline C15 & $5355.3(3)$ & $4673(2)$ & 6471.7(12) & $28.0(3)$ \\
\hline C16 & $5265.3(3)$ & $2930(2)$ & $5685.9(12)$ & $24.9(3)$ \\
\hline C17 & $5269.5(3)$ & $740(3)$ & $6205.9(12)$ & $28.7(3)$ \\
\hline C18 & $5598.3(3)$ & 293(2) & $6706.7(11)$ & $25.9(3)$ \\
\hline C19 & $5830.8(3)$ & 293(2) & $5887.4(11)$ & $22.0(3)$ \\
\hline C20 & $5688.1(4)$ & 2032(3) & 7497.0(11) & $28.2(3)$ \\
\hline N1 & $8348.2(3)$ & $3716(2)$ & 7343.2(9) & $28.3(3)$ \\
\hline N2 & $7098.3(3)$ & 2614.1(17) & $5823.0(8)$ & $16.8(2)$ \\
\hline N3 & $6385.3(3)$ & $2230.9(18)$ & $4876.5(9)$ & $19.9(3)$ \\
\hline $\mathrm{O} 1$ & $8505.6(3)$ & 2124(2) & $7565.8(9)$ & $37.1(3)$ \\
\hline $\mathrm{O} 2$ & $8440.4(3)$ & $5561(2)$ & 7492.1(10) & $46.8(3)$ \\
\hline $\mathrm{O} 3$ & $7016.6(2)$ & $8041.2(15)$ & 5742.2(7) & $22.1(2)$ \\
\hline
\end{tabular}




\begin{tabular}{lllrr} 
Atom & $x$ & $y$ & $z$ & $U(e q)$ \\
\hline O4 & $6336.7(2)$ & $7494.0(16)$ & $4655.3(8)$ & $25.7(2)$
\end{tabular}

Table S9. Hydrogen Atom Coordinates $\left(\AA \times 10^{4}\right)$ and Isotropic Displacement Parameters $\left(\AA^{2} \times 10^{3}\right)$ for 3 .

\begin{tabular}{l|rrrr} 
Atom & \multicolumn{1}{l}{$\boldsymbol{x}$} & \multicolumn{1}{c}{$\boldsymbol{y}$} & $\boldsymbol{U}(\mathrm{eq})$ \\
\hline H21A & 5495.56 & 4364.86 & 4535.7 & 26 \\
H21B & 5440.72 & 1848.21 & 4354.9 & 26 \\
H2 & 7966.71 & 6515.62 & 6451.93 & 25 \\
H3 & 7449.39 & 6101.21 & 5784.97 & 24 \\
H5 & 7487.08 & -201.66 & 6561.69 & 22 \\
H6 & 8010.02 & 209.01 & 7173.89 & 25 \\
H11A & 5992.76 & 1389.17 & 4018.64 & 25 \\
H11B & 6031.8 & 3922.15 & 4155.05 & 25 \\
H13A & 6130.81 & 3923.9 & 6489.72 & 24 \\
H13B & 5921.59 & 5639.25 & 5838.07 & 24 \\
H14 & 5741.42 & 5358.29 & 7487.21 & 29 \\
H15A & 5202.56 & 4695.49 & 6993.3 & 34 \\
H15B & 5351.87 & 6092.45 & 6136.9 & 34 \\
H16 & 5050.95 & 3223.8 & 5361.98 & 30 \\
H17A & 5210.42 & -388.22 & 5699.89 & 34 \\
H17B & 5116.16 & 721.07 & 6725.62 & 34 \\
H18 & 5601.07 & -1138.94 & 7046.26 & 31 \\
H19A & 5772.34 & -832.79 & 5379.87 & 26 \\
H19B & 6042.97 & -31.29 & 6199.45 & 26 \\
H20A & 5538.58 & 2024.6 & 8028.42 & 34 \\
H20B & 5899.5 & 1741.3 & 7824.24 & 34 \\
H2A & 7031.36 & 1281.3 & 5819.05 & 20 \\
H3A & 6459.74 & 921.99 & 4952.53 & 24
\end{tabular}




\section{Proton NMR Titration Anion Binding Studies}

\subsection{Overview and Procedures}

Proton NMR titrations were performed on a Bruker Avance DPX 400 spectrometer. For NMR titrations with chloride, a constant host concentration was maintained $(2.0 \mathrm{mM})$ using a prepared host solution to dissolve the guest (as $\mathrm{TBACl}$ ) to make the guest stock solution. Over the course of the titration, Hamiltonian Microlitre syringes were used to add aliquots of the guest stock solution to the NMR sample of the host solution. Chloride was added as the tetrabutylammonium (TBA) salt, purchased from Merck, after being dried under high vacuum $(<1.0 \mathrm{mmHg})$ for $24 \mathrm{~h}$. Stock solutions of the host were prepared in a relevant solution to the experiment. The host stock solutions $(600 \mu \mathrm{L})$ were transferred to an air-tight screwcap NMR sample tube (5 mm ID), and the same host stock solution was used to prepare the standard guest titrant solutions containing either $0.1 \mathrm{M}$, or $1 \mathrm{M}$ of TBACl. This ensured a constant concentration of the host for the duration of the titration experiment.

Over the course of the titration, small aliquots $(2-200 \mu \mathrm{L})$ of the standard guest solution were added to the host solution in the NMR tube. For each titration, 16-20 data points were collected. The number of equiv. of guest present at the end of each titration is labelled in the stacked plots. Before collecting each data point, an aliquot of the standard guest solution was added, and the sample was thoroughly mixed in the NMR tube. The system was allowed to equilibrate for up to $2 \mathrm{~min}$ inside the NMR probe before the spectra was collected. Throughout each titration experiment, all parameters of the NMR spectrometer remained constant.

In all cases, the proton resonances were monitored for changes in chemical shift. When possible, two or more resonances were followed, allowing several data sets to determine the association constant (Ka). Global fitting takes into account all data sets at the same time and improves the quality of the non-linear curve fitting. The supramolecular.org web applet was used to fit the titration data with chloride to a 1:1 model. ${ }^{1}$ 


\subsection{Titration Data with TBACl Guest}

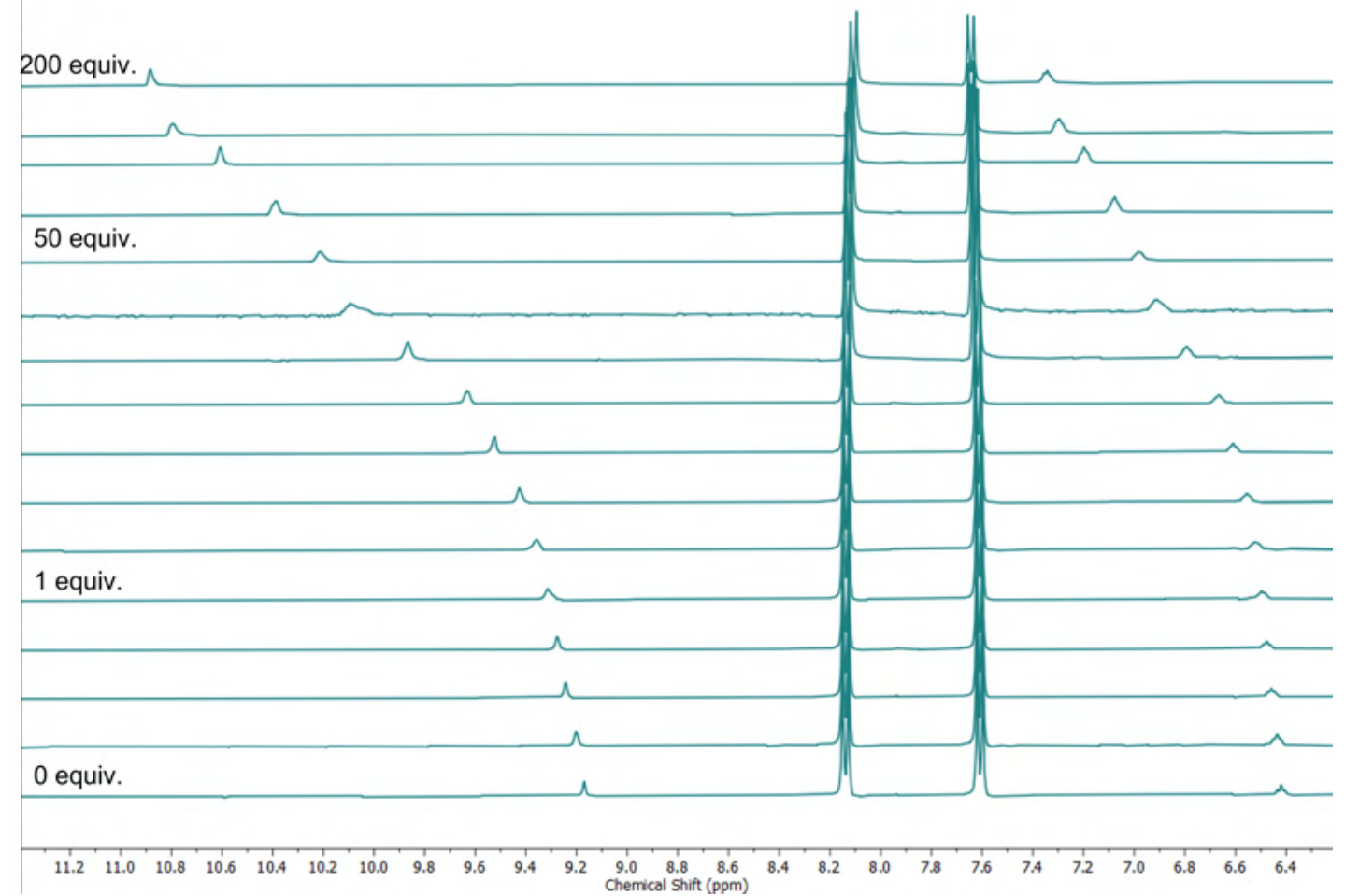

Figure S32. ${ }^{1} \mathrm{H}$ NMR titration spectra as a stack plot for $\mathbf{1}(2 \mathrm{mM})+\mathrm{TBACl}(0-200$ equiv. $)$ in DMSO-d $6 / 0.5 \% \mathrm{D}_{2} \mathrm{O}$ at $298 \mathrm{~K}$.
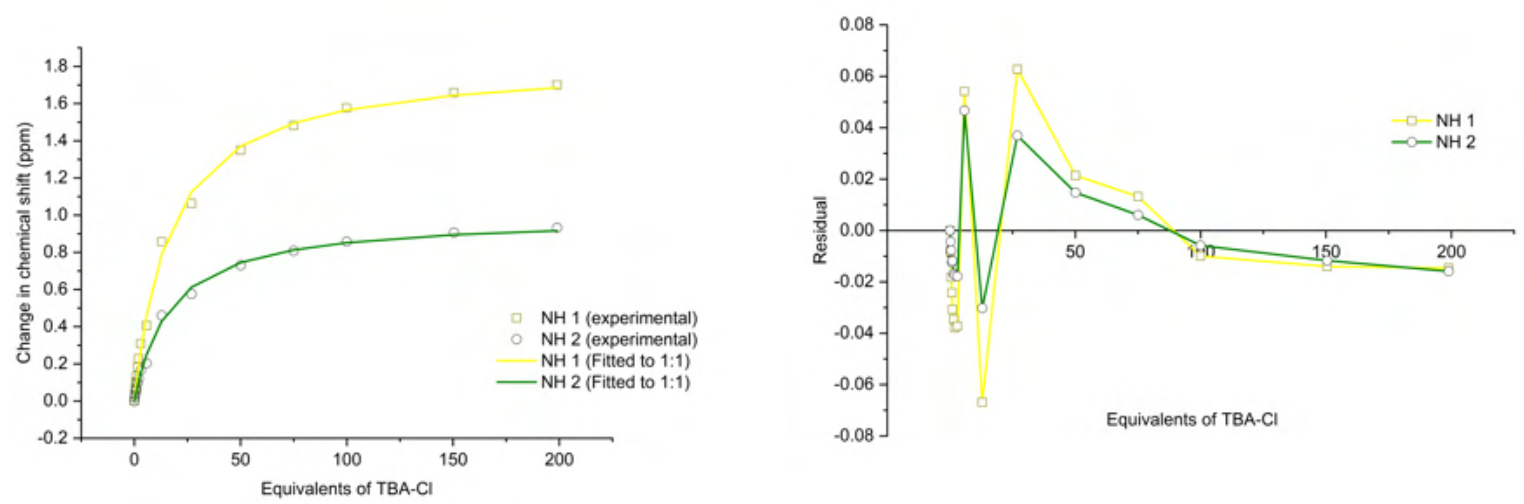

Figure S33. Fitted binding isotherm of $\mathbf{1}+\mathrm{TBACl}$ showing the change in chemical shift of $\mathrm{NH}$ protons fitted to the $1: 1$ binding model (left). $K_{\mathrm{a}}=33.69 \mathrm{M}^{-1}$. Residual plot showing the random error obtained from the binding isotherm fitting (right). Covariance of fit $(\operatorname{cov} f i t)=2.51 \times 10^{-3}$. Link to Bindfit fitting: http://app.supramolecular.org/bindfit/view/3d7ecc84-f990-4b32-bd2584853ea2c174. 


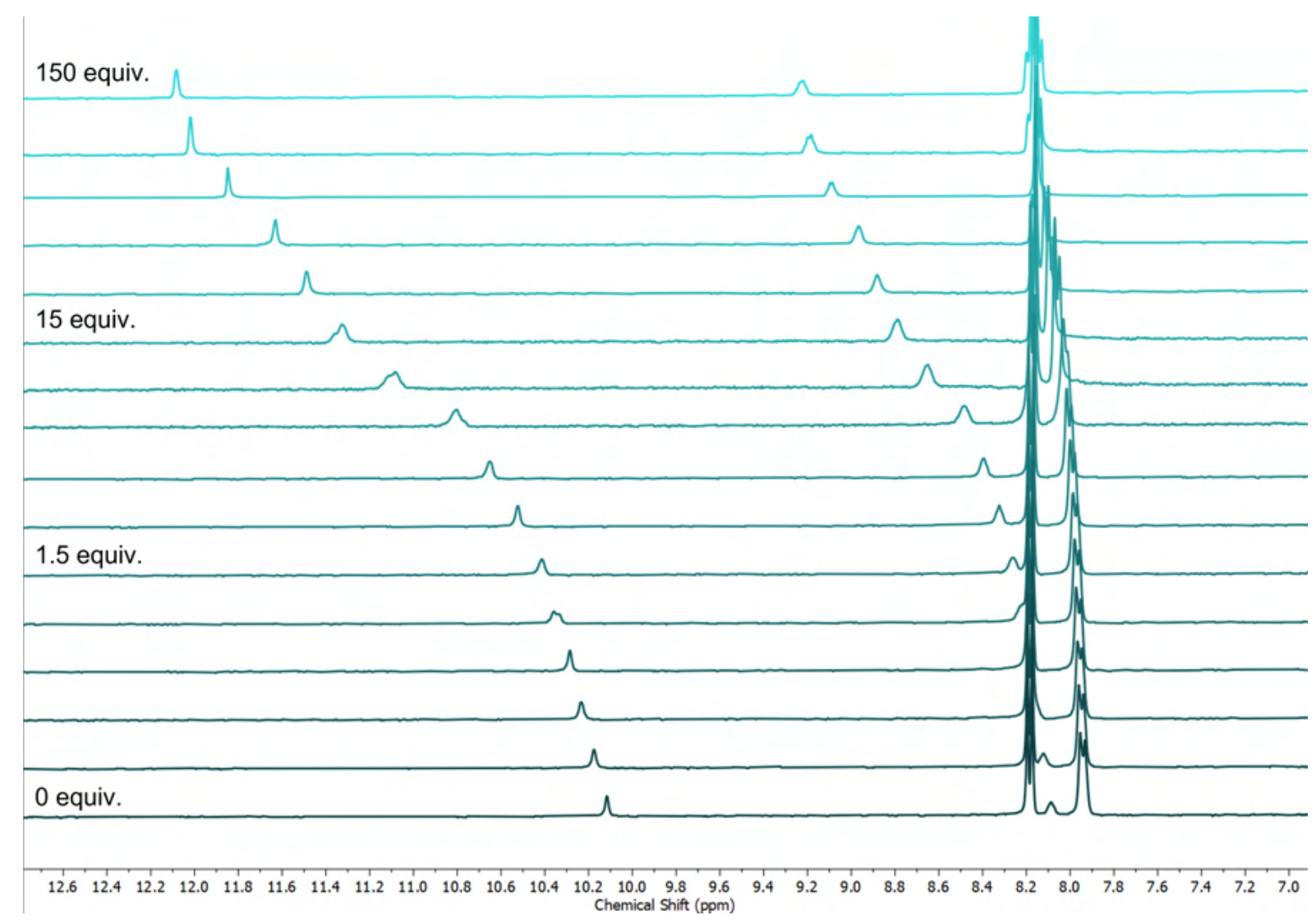

Figure S34. ${ }^{1} \mathrm{H}$ NMR titration spectra as a stack plot for $2(2 \mathrm{mM})+\mathrm{TBACl}(0-150$ equiv. $)$ in DMSO-d $6 / 0.5 \% \mathrm{D}_{2} \mathrm{O}$ at $298 \mathrm{~K}$.
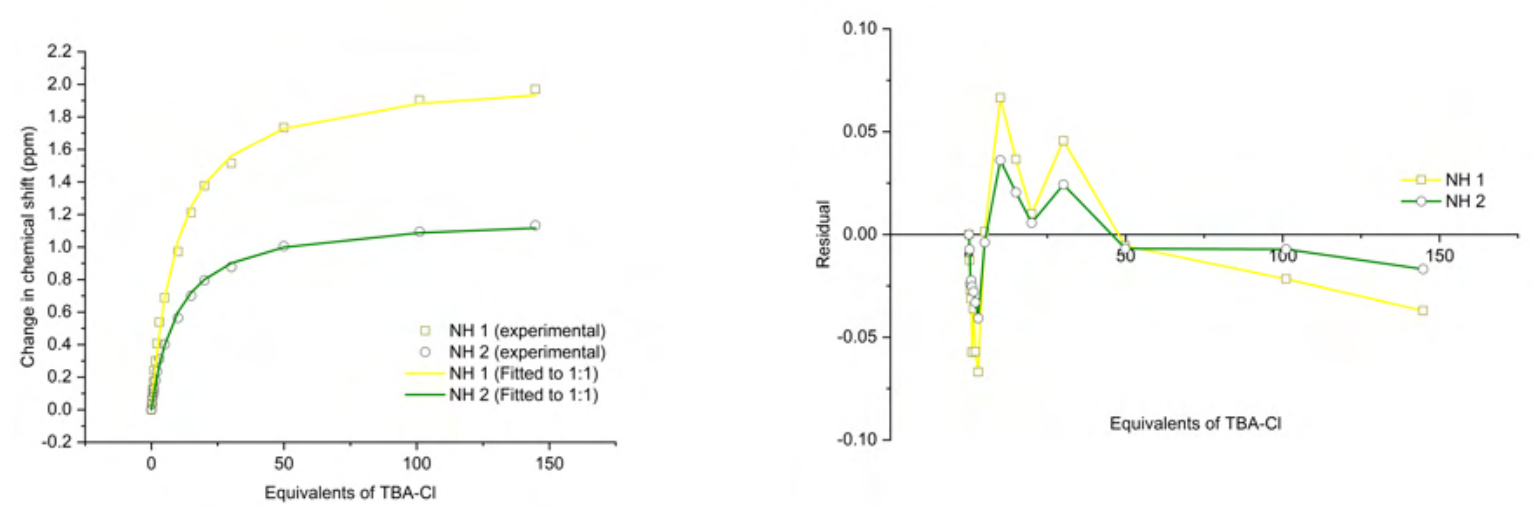

Figure S35. Fitted binding isotherm of $2+\mathrm{TBACl}$ showing the change in chemical shift of the $\mathrm{NH}$ protons fitted to the 1:1 binding model (left). $K_{\mathrm{a}}=47.84 \mathrm{M}^{-1}$. Residual plot showing the random error obtained from the binding isotherm fitting (right). Covariance of fit (covfit) $=2.69$ x 10-3. Link to Bindfit fitting: http://app.supramolecular.org/bindfit/view/0b1005ac-fdd7$\underline{4 d 45-9405-193794 f c 5777 .}$. 


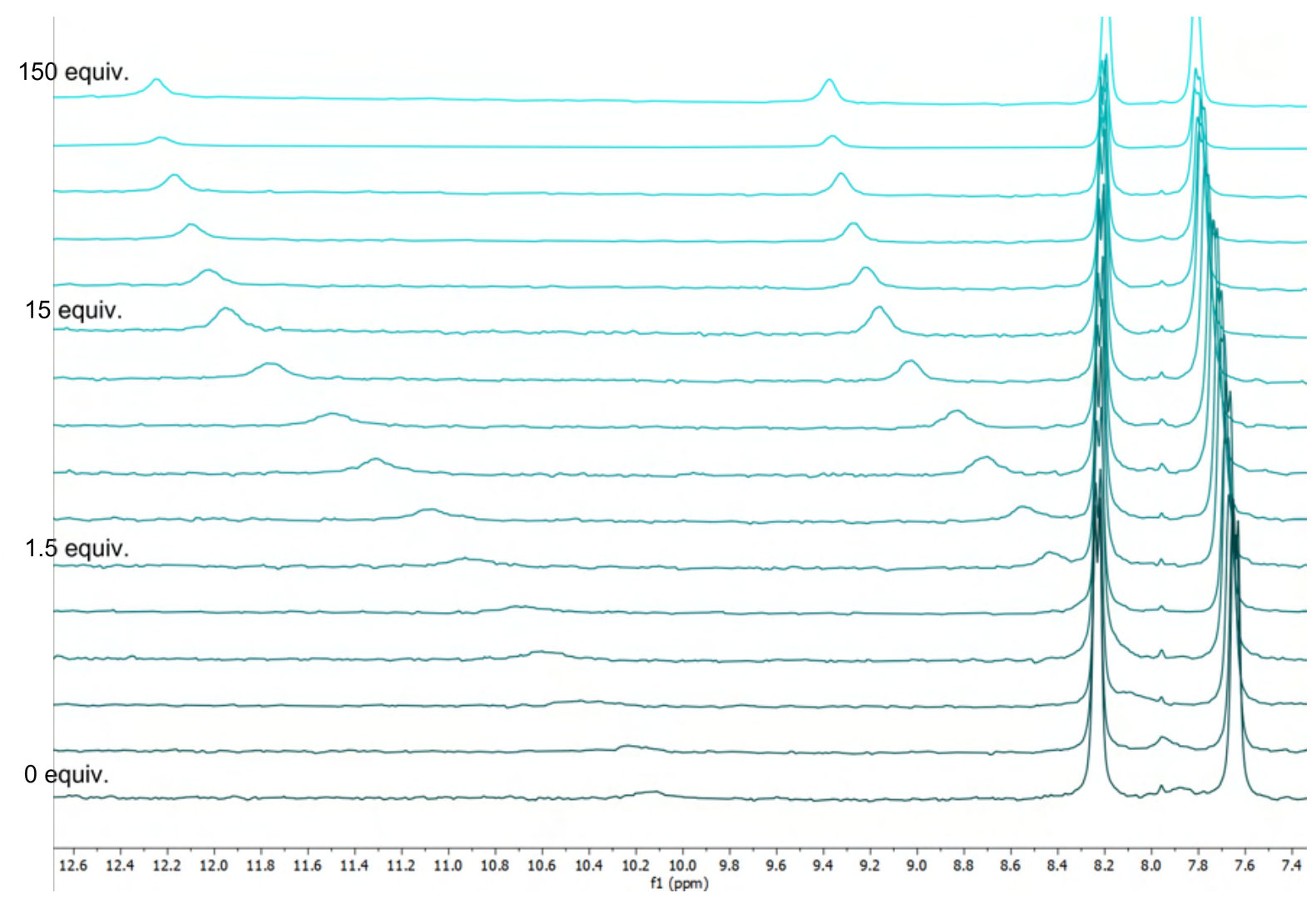

Figure S36. ${ }^{1} \mathrm{H}$ NMR titration spectra as a stack plot for $3(2 \mathrm{mM})+\mathrm{TBACl}(0-150$ equiv. $)$ in DMSO-d $6 / 0.5 \% \mathrm{D}_{2} \mathrm{O}$ at $298 \mathrm{~K}$.
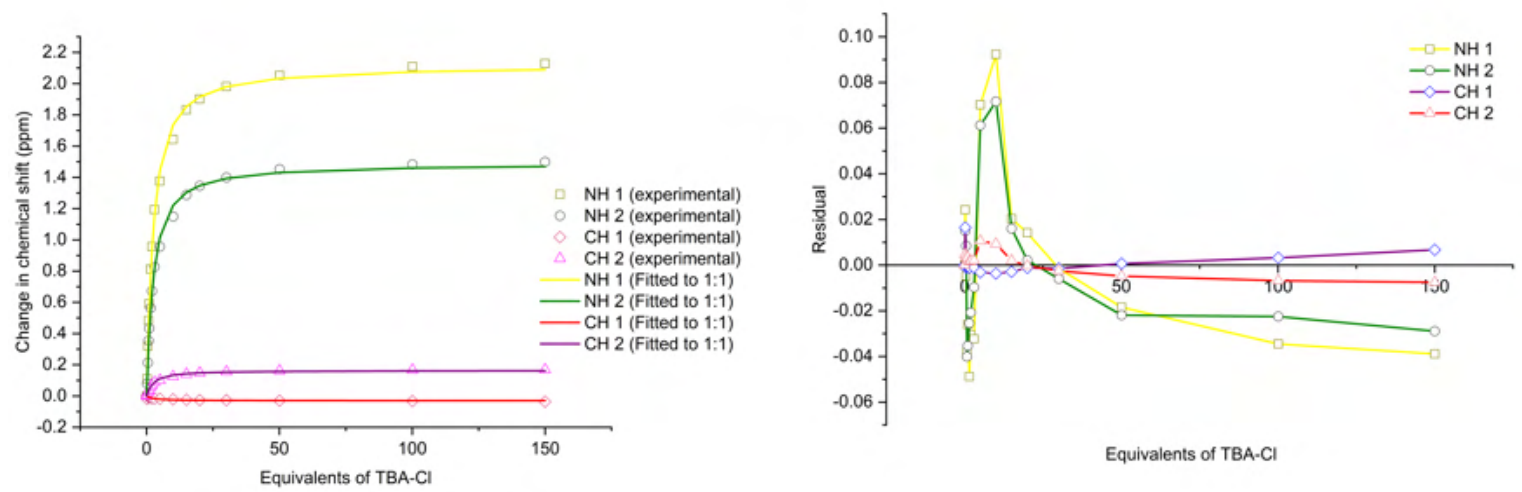

Figure S37. Fitted binding isotherm of $3+\mathrm{TBACl}$ showing the change in chemical shift of $\mathrm{NH}$ and aromatic $\mathrm{CH}$ protons fitted to the $1: 1$ binding model (left). $K_{\mathrm{a}}=249.09 \mathrm{M}^{-1}$. Residual plot showing the random error obtained from the binding isotherm fitting (right). Covariance of fit $\left(\operatorname{cov}_{\text {fit }}\right)=1.34 \quad \mathrm{x} \quad 10^{-3}$. Link to Bindfit fitting: http://app.supramolecular.org/bindfit/view/1c356b9b-999a-4412-9c0e-31ceadb72649. 


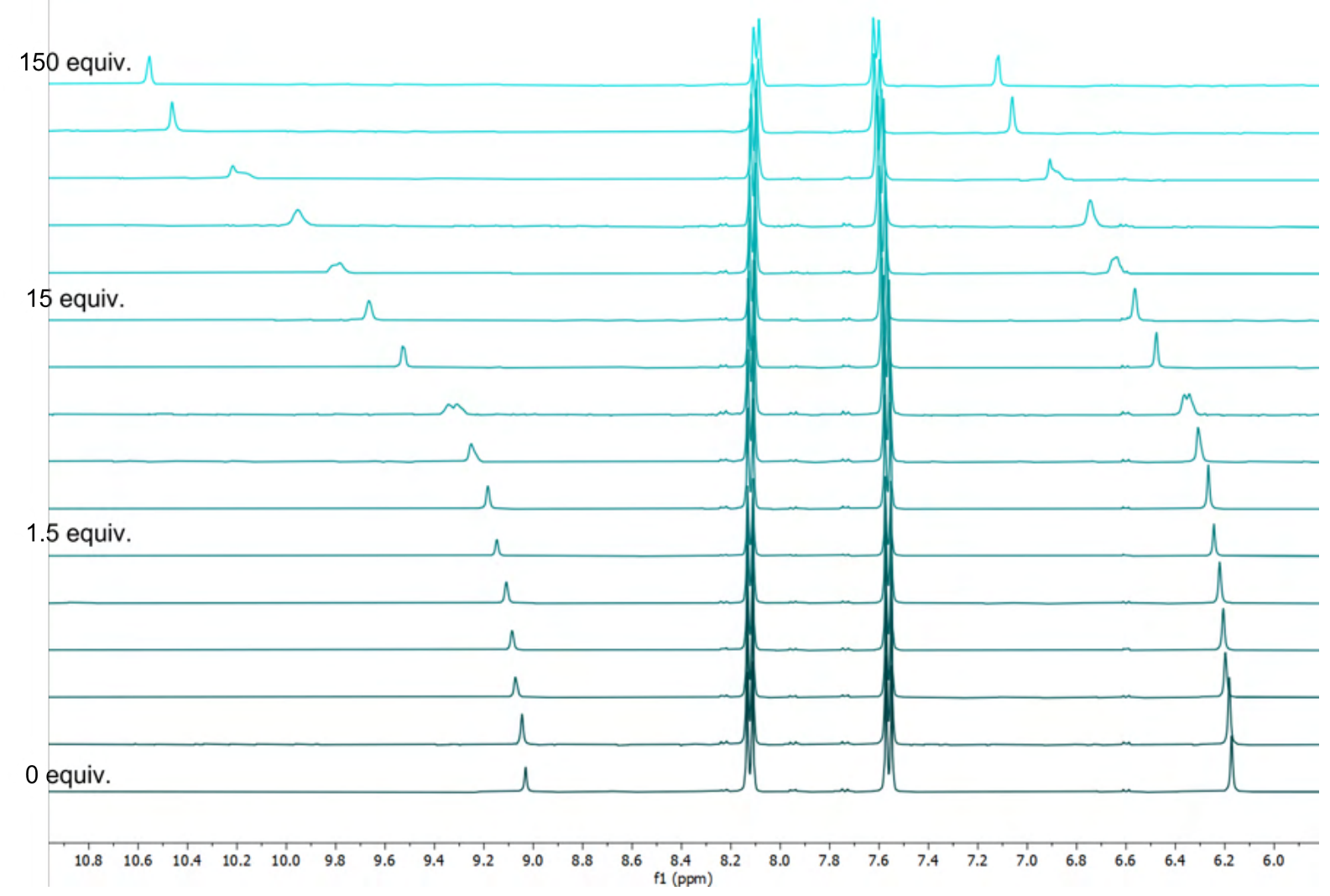

Figure S38. ${ }^{1} \mathrm{H}$ NMR titration spectra as a stack plot for $4(2 \mathrm{mM})+\mathrm{TBACl}(0-150$ equiv. $)$ in DMSO-d $6 / 0.5 \% \mathrm{D}_{2} \mathrm{O}$ at $298 \mathrm{~K}$.
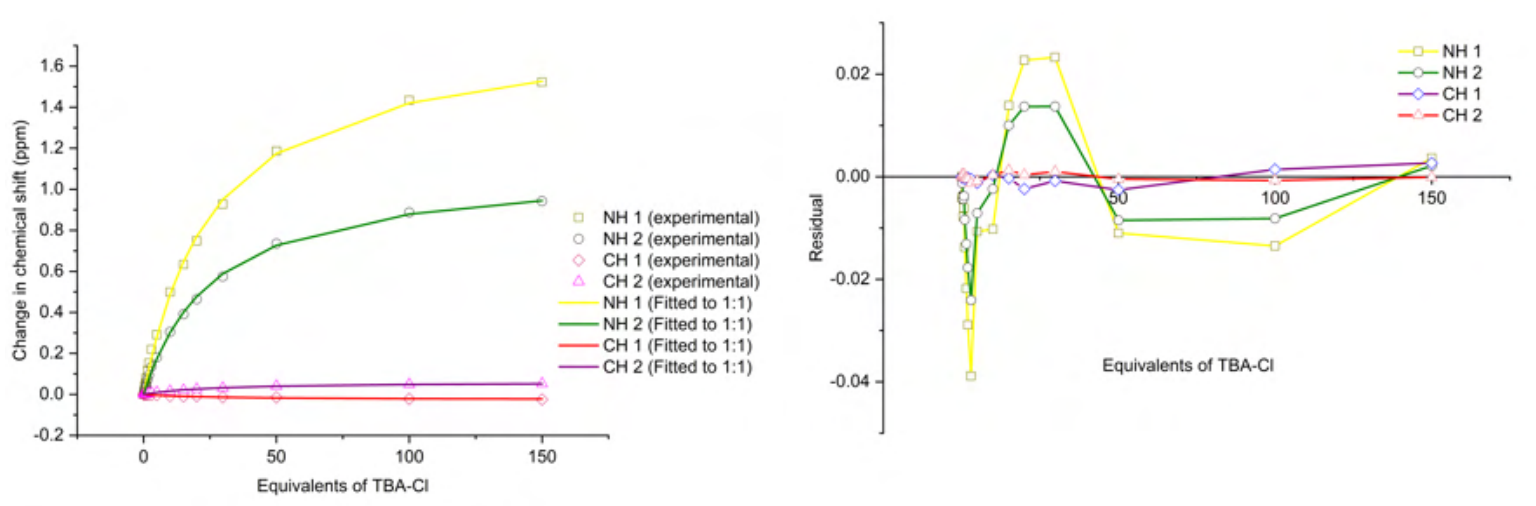

Figure S39. Fitted binding isotherm of $4+\mathrm{TBACl}$ showing the change in chemical shift of $\mathrm{NH}$ and aromatic $\mathrm{CH}$ protons fitted to the $1: 1$ binding model (left). $K_{\mathrm{a}}=21.29 \mathrm{M}^{-1}$. Residual plot showing the random error obtained from the binding isotherm fitting (right). Covariance of fit $\left(\operatorname{cov}_{\text {fit }}\right) \quad=7.48 \quad \mathrm{x} \quad 10^{-4}$. Link to Bindfit fitting: http://app.supramolecular.org/bindfit/view/db17772a-eff5-4005-975d-5d5dbca35bf5. 


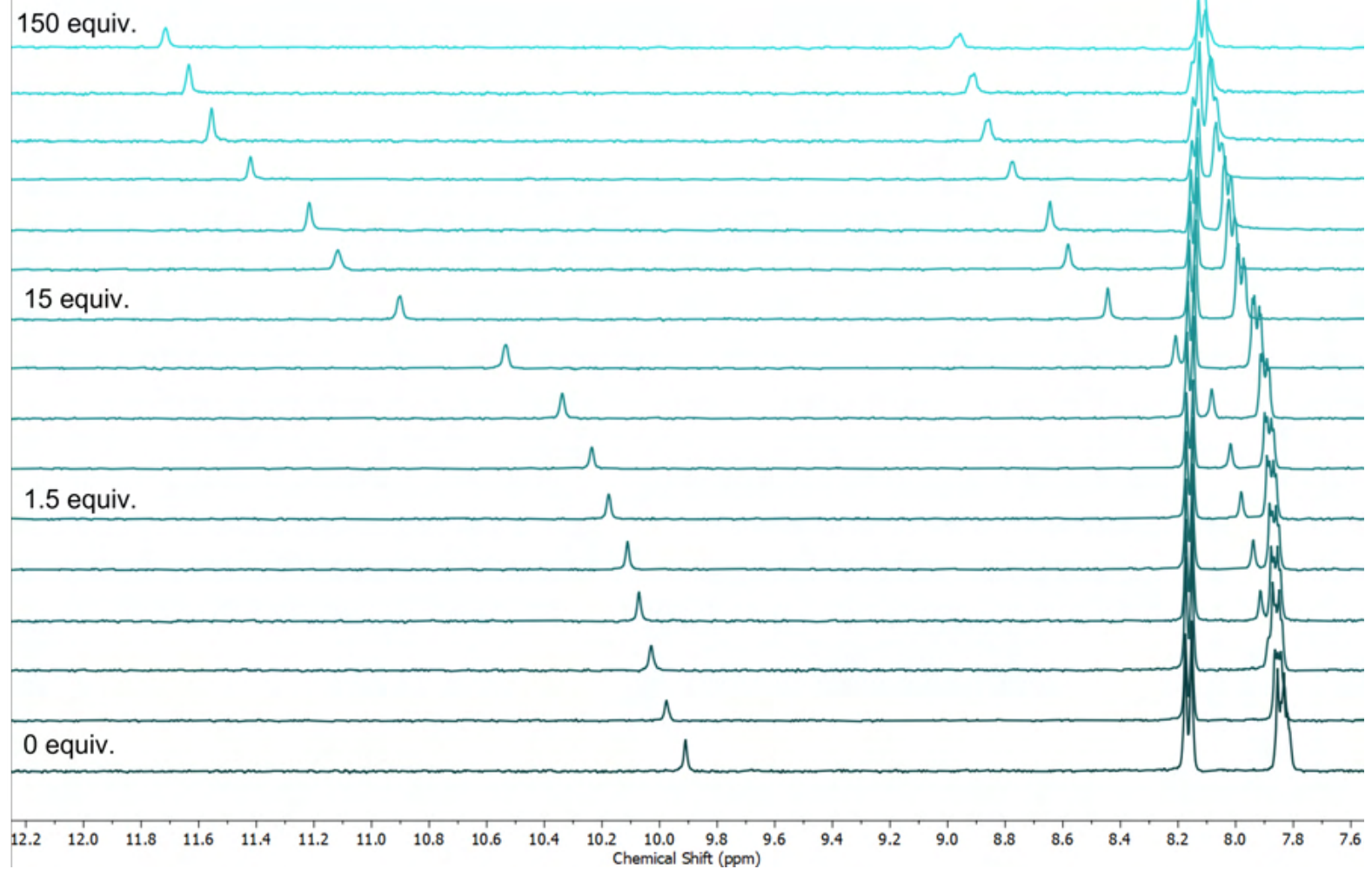

Figure S40. ${ }^{1} \mathrm{H}$ NMR titration spectra as a stack plot for $5(2 \mathrm{mM})+\mathrm{TBACl}(0-150$ equiv. $)$ in DMSO-d $6 / 0.5 \% \mathrm{D}_{2} \mathrm{O}$ at $298 \mathrm{~K}$.
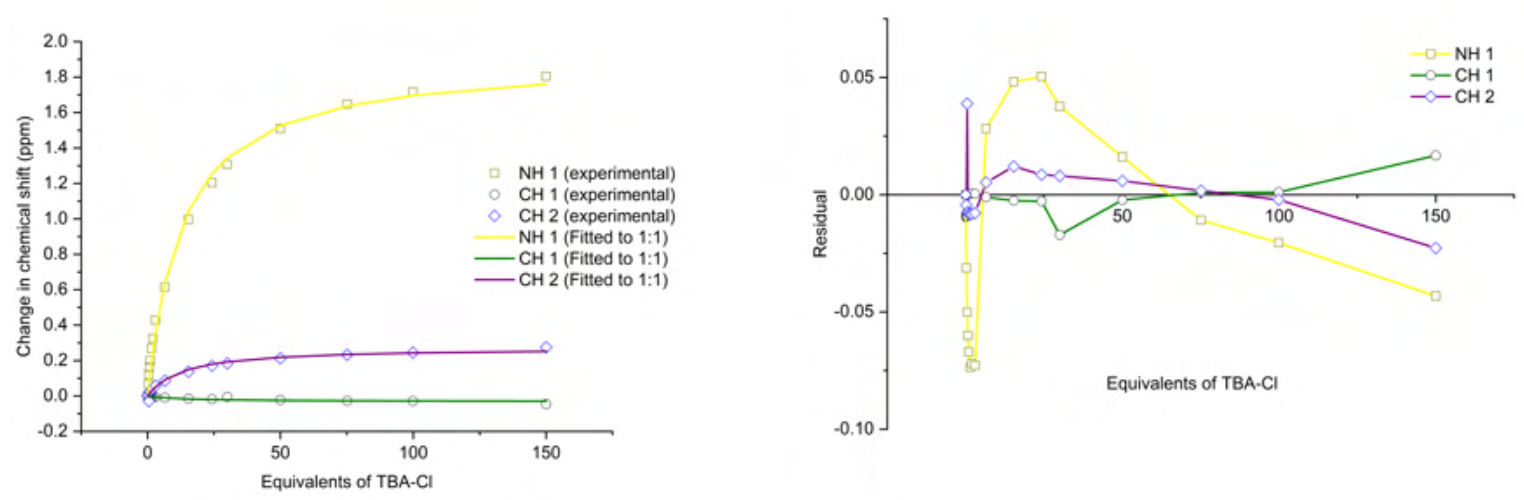

Figure S41. Fitted binding isotherm of $5+\mathrm{TBACl}$ showing the change in chemical shift of a $\mathrm{NH}$ proton and 2 aromatic $\mathrm{CH}$ protons fitted to the 1:1 binding model (left). $K_{\mathrm{a}}=38.56 \mathrm{M}^{-1}$. Residual plot showing the random error obtained from the binding isotherm fitting (right). Covariance of fit $\left(\operatorname{cov}_{\mathrm{fit}}\right)=3.08 \times 10^{-3}$. Link to Bindfit fitting: http://app.supramolecular.org/bindfit/view/37a72349-1087-4b8d-a0db-6a6f226d9a59. 


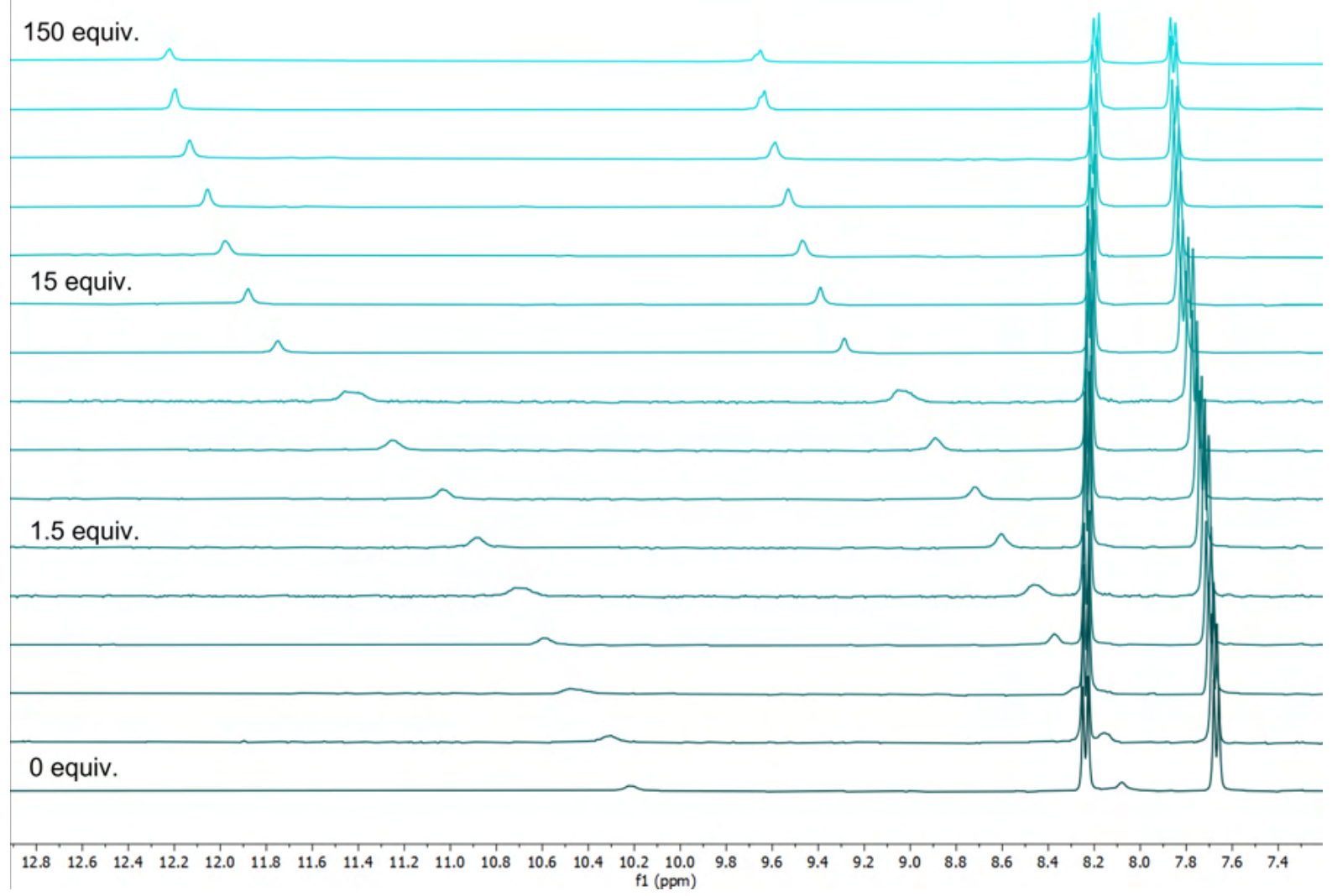

Figure S42. ${ }^{1} \mathrm{H}$ NMR titration spectra as a stack plot for $6(2 \mathrm{mM})+\mathrm{TBACl}(0-150$ equiv. $)$ in DMSO-d $6 / 0.5 \% \mathrm{D}_{2} \mathrm{O}$ at $298 \mathrm{~K}$.
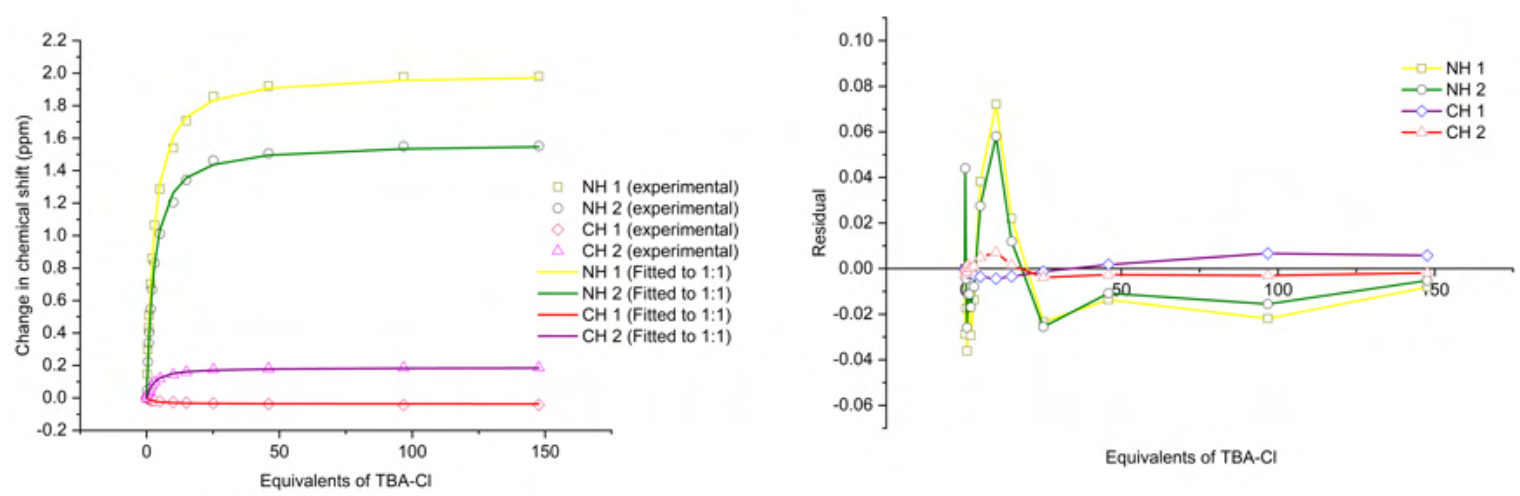

Figure S43. Fitted binding isotherm of $6+\mathrm{TBACl}$ showing the change in chemical shift of $\mathrm{NH}$ and aromatic $\mathrm{CH}$ protons fitted to the 1:1 binding model (left). $K_{\mathrm{a}}=211.22 \mathrm{M}^{-1}$. Residual plot showing the random error obtained from the binding isotherm fitting (right). Covariance of fit $\left(\operatorname{cov}_{\text {fit }}\right)=8.35 \quad \mathrm{x} \quad 10^{-4}$. Link to Bindfit fitting: http://app.supramolecular.org/bindfit/view/cceb5ef1-3e56-443e-af5a-d66fe42b2a26. 


\section{Inclusion Complex Preparation}

The most energetically stable configuration of the cyclodextrins takes the form of a conical cylinder, with a slightly larger opening on the face containing the secondary hydroxyl groups. ${ }^{2}$ The internal cavity contains multiple hydrogen atoms and glycosidic oxygen bridges, which create an apolar, hydrophobic microenvironment, whilst the multitude of hydroxyl groups situated on the exterior of the cavity creates a hydrophilic rim on either end of the cyclodextrin cylinder. The ${ }^{1} \mathrm{H}$ NMR spectrum of HP- $\beta-C D$ is shown in Figure S44 with the internal and external protons labelled. The contrasting properties of the hydrophilic outer surface and the hydrophobic inner cavity of cyclodextrins means the macrocycles possess the ability to act as molecular containers for non-polar guests of an appropriate size. This interaction is purely supramolecular in nature, driven by van der Waals forces, host:guest hydrogen bonding interactions, and the dehydration of the non-polar internal cavity by the insertion of a guest molecule (hydrophobic effect). ${ }^{3}$ A solute can be fully or partially encapsulated by a CD host and, in solution, the free $C D$, free guest, and inclusion complex maintain a dynamic equilibrium. The most important consequence of this encapsulation is an increase in the aqueous solubility of the guest molecule.

Cyclodextrin inclusion complex strategies have been widely implemented to improve the aqueous solubility and bioavailability of drug molecules. ${ }^{4} \mathrm{CDs}$ are available on an industrial scale from a renewable natural resource and are recognised as non-toxic. ${ }^{5-7}$ The complexation of drugs can also reduce irritation to the stomach or skin by reducing the local concentration of the molecule, mask an unpleasant odour or taste, and prevent the crystallisation of active ingredients. ${ }^{8-10}$ 

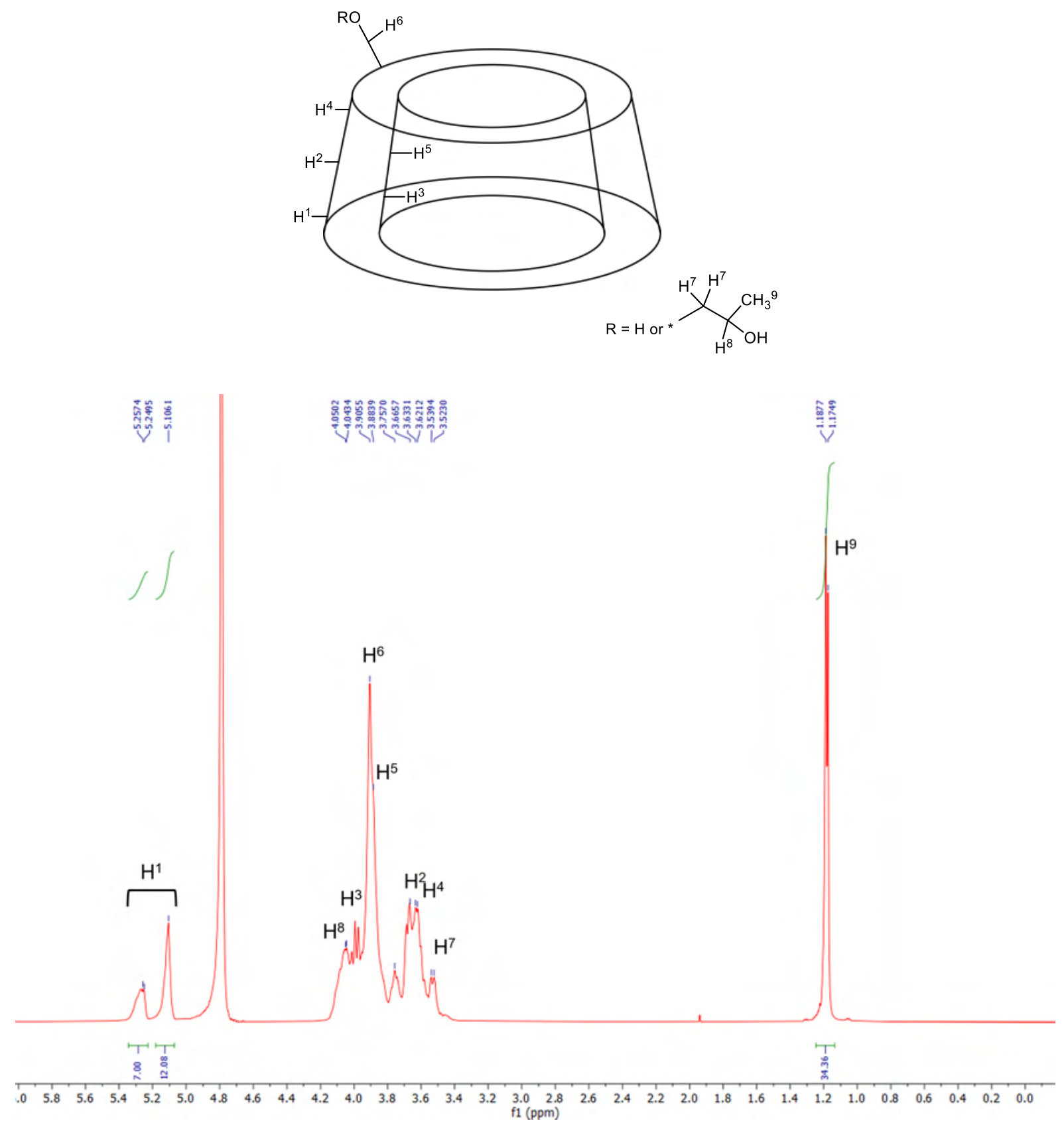

Figure S44. The ${ }^{1} \mathrm{H}$ NMR spectrum (500 MHz) for $\mathrm{HP}-\beta-\mathrm{CD}$ in $\mathrm{D}_{2} \mathrm{O}$. The signals attributed to each proton are labelled and correspond to the diagram of HP- $\beta$-CD diagram above, adapted from the literature. ${ }^{11}$ 


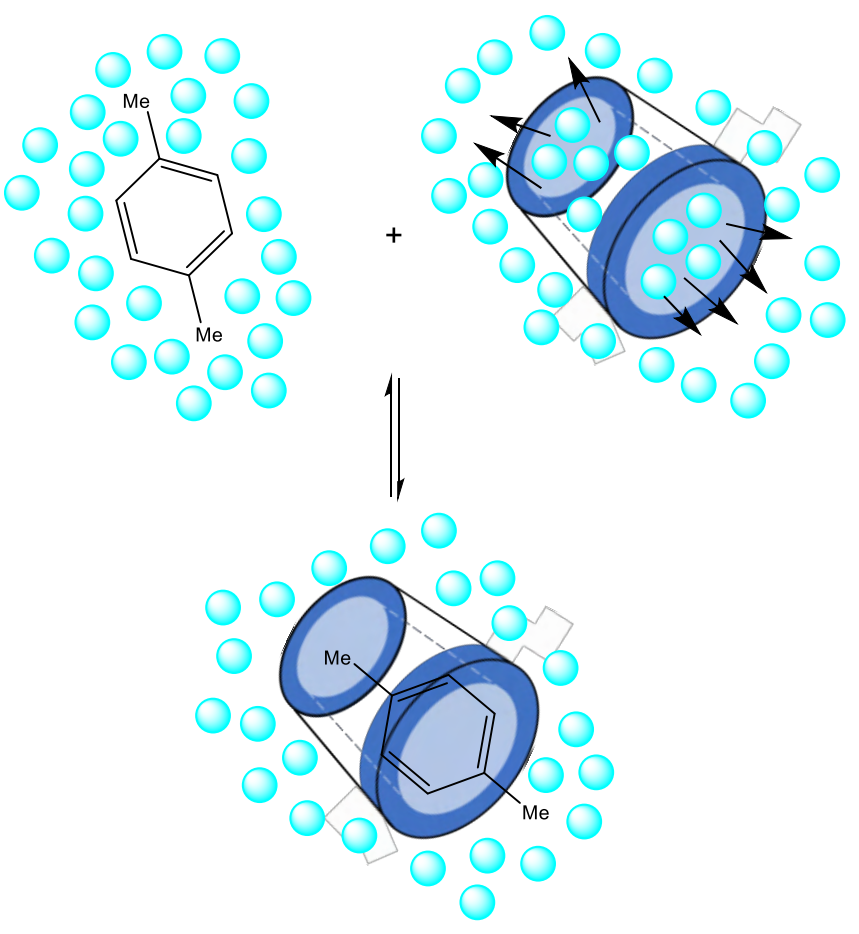

Figure S45. Schematic representation of the CD inclusion complex equilibrium adapted from the literature. ${ }^{2} p$-Xylene is the guest molecule, and the water molecules (blue spheres) are excluded from the hydrophobic cavity of the cyclodextrin host during the inclusion process.

The formation of an inclusion complex between HP- $\beta-C D$ and each of the compounds 1-6 was attempted using three different methods, adapted from procedures employed in the literature. ${ }^{12,13}$ Each method involved using a 1:1 water:ethanol solvent mixture to ensure that both host and guest were partially solubilised during the process and can reorientate to form an inclusion complex. Additionally, each inclusion complex mixture was added to pure water after the completion of the inclusion process. This addition resulted in the precipitation of any uncomplexed transporter, which was filtered and removed, leaving a filtrate containing a mixture of free HP- $\beta-\mathrm{CD}$ host and the desired inclusion complex. A total of 24 inclusion complex procedures were attempted.

\subsection{Method 1: Kneading}

An equimolar physical mixture of HP- $\beta$-CD (138 mg, $0.01 \mathrm{mmol})$ and transporter $(0.01 \mathrm{mmol})$ was combined with a mortar and pestle. A small volume $(\sim 0.5-1.0 \mathrm{~mL})$ of 1:1 water:ethanol solvent mixture was added dropwise, and the components were ground together into a homogenous paste for $30 \mathrm{~min}$. Additional solvent was added when required to keep the paste from drying out. Following this, the product was dried at $65{ }^{\circ} \mathrm{C}$ in a vacuum oven for $2 \mathrm{~h}$ to 
remove residual solvent. The dry powder was transferred to a glass vial $(21 \mathrm{~mL})$, and a portion of Milli-q water $(5 \mathrm{~mL})$ was added. The resulting precipitate was filtered out, and the filtrate was collected. The solvent was removed under nitrogen before the solid residue was dried overnight in vacuo yielding a crystalline solid.

\subsection{Method 2: Microwave Irradiation (Minimum Solvent)}

An equimolar physical mixture of HP- $\beta-\mathrm{CD}(138 \mathrm{mg}, 0.01 \mathrm{mmol})$ and transporter $(0.01 \mathrm{mmol})$ was combined with a mortar and pestle. In the absence of solvent, the two components were ground together for $10 \mathrm{~min}$ to form a homogenous and amorphous solid mixture. The two components were transferred to a $10 \mathrm{~mL}$ Pyrex microwave vessel, and a $w / w$ mass of $1: 1$ water:ethanol solvent mixture was added (approx. $200 \mathrm{mg}$ ). The mixture was sonicated to ensure that all the powder mixture was saturated by solvent. Next, the reaction vessel was subjected to microwave irradiation for $10 \mathrm{~min}$ using a single-mode CEM Discover ${ }^{\circledR}$ microwave synthesiser kept at $80{ }^{\circ} \mathrm{C}$ through moderation of the initial microwave power $(150$ W). Subsequently, the product was dried at $65^{\circ} \mathrm{C}$ in a vacuum oven for $2 \mathrm{~h}$ to remove residual solvent. The dry powder was transferred to a glass vial $(21 \mathrm{~mL})$, and a portion of Milli-q water $(5 \mathrm{~mL})$ was added. The resultant precipitate was filtered and removed whilst the filtrate was collected. The solvent was removed under nitrogen before being dried overnight in vacuo to yield a crystalline solid.

\subsection{Method 3: Microwave Irradiation (2.5 mL of Solvent)}

This method was adapted from the procedure outlined by Noto and co-workers. ${ }^{13}$ An equimolar physical mixture of $\mathrm{HP}-\beta-\mathrm{CD}(138 \mathrm{mg}, 0.01 \mathrm{mmol})$ and transporter $(0.01 \mathrm{mmol})$ was combined with a mortar and pestle, ground together for $10 \mathrm{~min}$ to form a homogenous and amorphous solid mixture in the absence of solvent. The two components were transferred to a $10 \mathrm{~mL}$ Pyrex vessel, and a portion of 1:1 water:ethanol solvent mixture $(2.5 \mathrm{~mL})$ was added. The mixture was sonicated to ensure that all the powder mixture was saturated by solvent and not stuck to the walls of the vessel. Next, the reaction vessel was subjected to microwave irradiation for 25 min using a single-mode CEM Discover® microwave synthesiser kept at 80 ${ }^{\circ} \mathrm{C}$ through moderation of the initial microwave power $(150 \mathrm{~W})$. A portion of pure water $(5$ $\mathrm{mL}$ ) was added to the Pyrex vessel, and the resultant precipitate was filtered and removed 
whilst the filtrate was collected. The solvent was removed under nitrogen before being dried overnight in vacuo to yield a crystalline solid.

\section{Proton NMR Spectroscopy Analysis of Inclusion Complexes}

The water-soluble filtrates of all 24 inclusion attempts (Compounds 1-6, methods 1-3) were collected and dried to leave crystalline solids. Subsequently, a small portion of each solid (10-15 mg) was dissolved in $\mathrm{D}_{2} \mathrm{O}$, and a ${ }^{1} \mathrm{H}$ NMR spectrum $(400 \mathrm{MHz}$ ) was collected. Dissolution attempts in $\mathrm{D}_{2} \mathrm{O}$ of several solid mixtures resulted in the precipitation of a solid, which was removed before a spectrum was obtained. The solid obtained is likely free transporter and suggests that, for these attempts, an inclusion complex did not fully form or that the compounds decomplexed from HP- $\beta-C D$ as the water was removed during the last step of each method.

Crucial evidence supporting the existence of an inclusion complex is the appearance of resonance signals in the $\mathrm{D}_{2} \mathrm{O}$ spectrum that can be attributed to the protons of the transporter compound. Compounds 1-6 exhibit very low solubility in $\mathrm{D}_{2} \mathrm{O}$, and the appearance of signals associated with these molecules suggests that their aqueous solubility has been enhanced. The integrations of the resonances attributed to the transporter molecule relative to the HP- $\beta-C D$ signals provide an indication of how much of the transporter is present in the inclusion complex. However, the relative integrations cannot be used to calculate the exact stoichiometric ratios of transporter and $\mathrm{HP}-\beta-\mathrm{CD}$. Commercial $\mathrm{HP}-\beta-\mathrm{CD}$ is prepared via the condensation of native $\beta-C D$ with an excess of propylene oxide, but this does not result in the complete substitution of the hydroxyl groups. ${ }^{14}$ Therefore, HP- $\beta-C D$ typically contains a mixture of hydroxypropyl derivatives and free hydroxyl groups, and the supplier provides an average molecular weight (M.W. $1380 \mathrm{gmol}^{-1}$ ). This means that integration cannot be reliably determined and used to calculate the relative stoichiometry. In addition, the proximity of some signals to the water resonances means that the integration of these protons may correspond to a number larger than the true value. Nevertheless, the appearance of large and distinct resonance peaks attributed to the transporter compound is reliable evidence for the formation of an inclusion complex.

The ${ }^{1} \mathrm{H}$ NMR spectra of the inclusion complex formed between 1 and HP- $\beta$-CD using method 3 in $\mathrm{D}_{2} \mathrm{O}$ is showed in Figure S46, and peaks attributed to compound $\mathbf{1}$ are clearly visible. The 
aromatic peaks at 8.2 and $7.7 \mathrm{ppm}$, the methyl spacer protons at $3 \mathrm{ppm}$, and adamantyl peaks at $2.2,1.8$, and $1.7 \mathrm{ppm}$ can all be distinguished and have the correct proportionality when integrated. The signals for the urea protons of $\mathbf{1}$ are not present in the spectrum, which is likely a consequence of the deuterium exchange of these acidic protons with the bulk solvent. Guest molecule resonances of appreciable intensity were distinguished in the ${ }^{1} \mathrm{H}$ NMR spectra, in $\mathrm{D}_{2} \mathrm{O}$, of HP- $\beta-\mathrm{CD} \cdot \mathbf{1}$ (methods $\left.1-3\right), \mathrm{HP}-\beta-\mathrm{CD} \cdot 2$ (methods $\left.1-3\right), \mathrm{HP}-\beta-\mathrm{CD} \cdot 4$ (methods $1-3$ ) and HP- $\beta$-CD.6 (methods 1-2). Proton NMR spectra for all inclusion attempts can be viewed below.

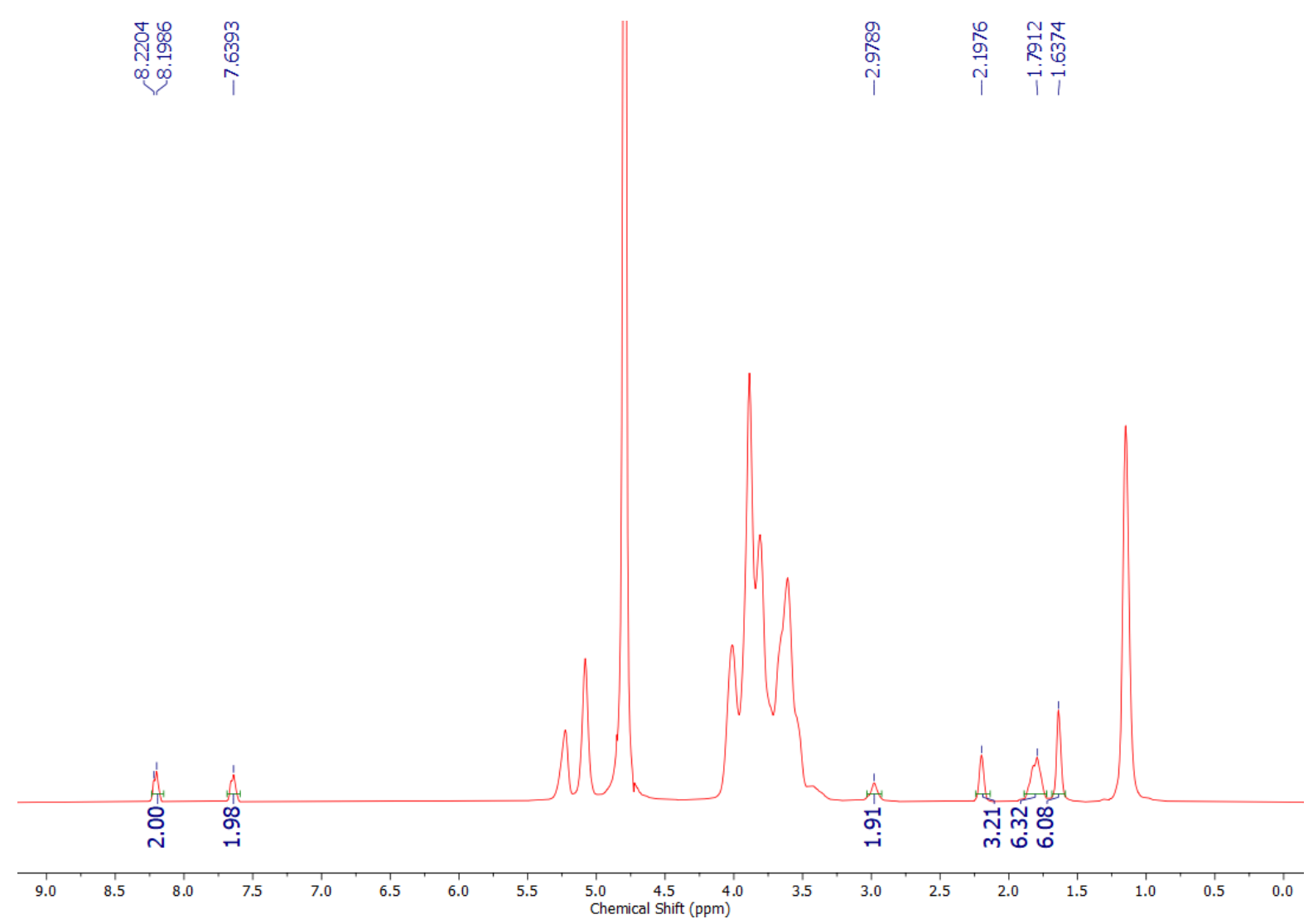

Figure S46. ${ }^{1} \mathrm{H}$ NMR $(400 \mathrm{MHz})$ spectrum of $\mathrm{HP}-\beta-\mathrm{CD} \cdot \mathbf{1}($ method 1$)$ in $\mathrm{D}_{2} \mathrm{O}$ at $298 \mathrm{~K}$. 


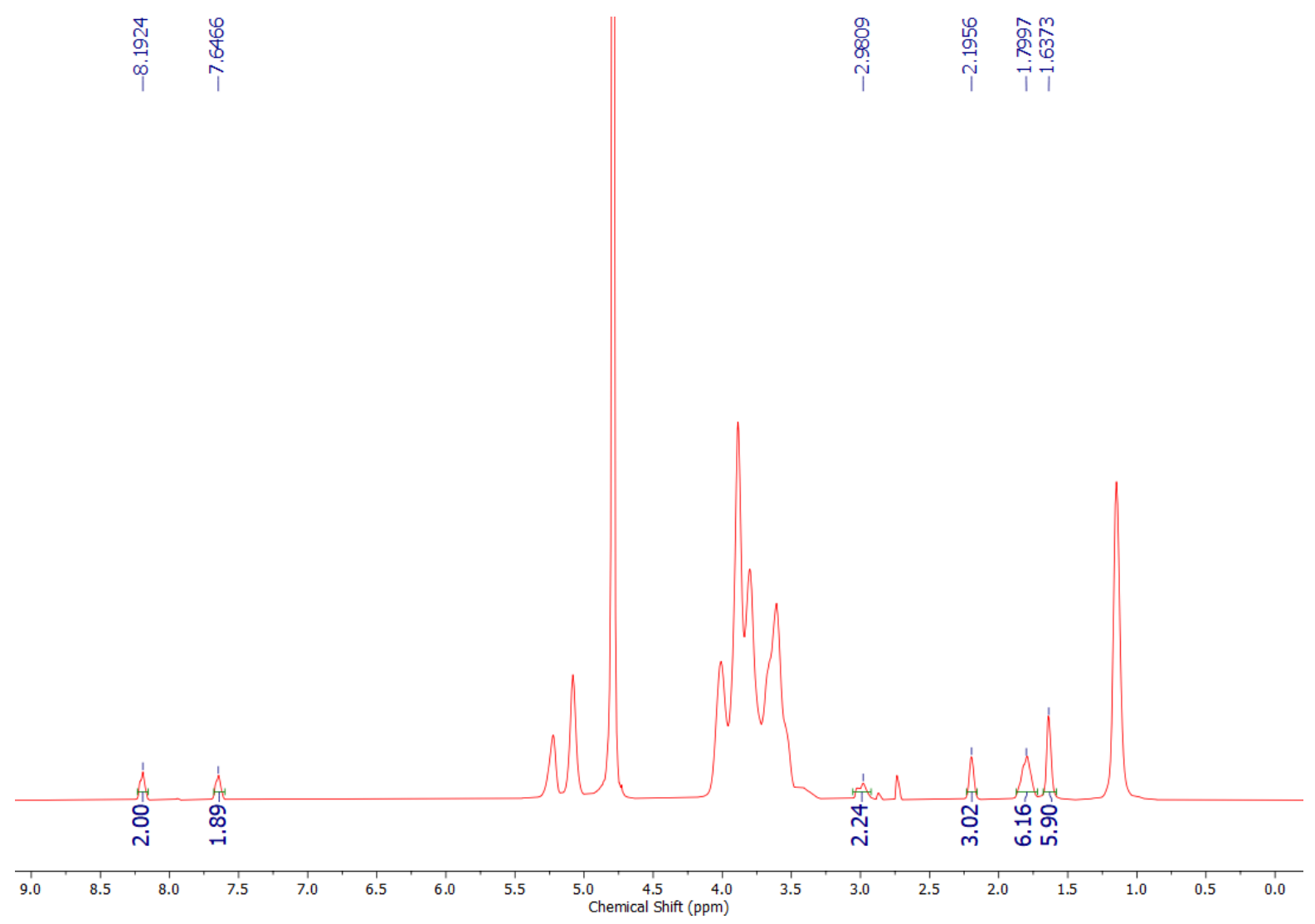

Figure S47. ${ }^{1} \mathrm{H}$ NMR $(400 \mathrm{MHz})$ spectrum of $\mathrm{HP}-\beta-\mathrm{CD} \cdot \mathbf{1}($ method 2$)$ in $\mathrm{D}_{2} \mathrm{O}$ at $298 \mathrm{~K}$.

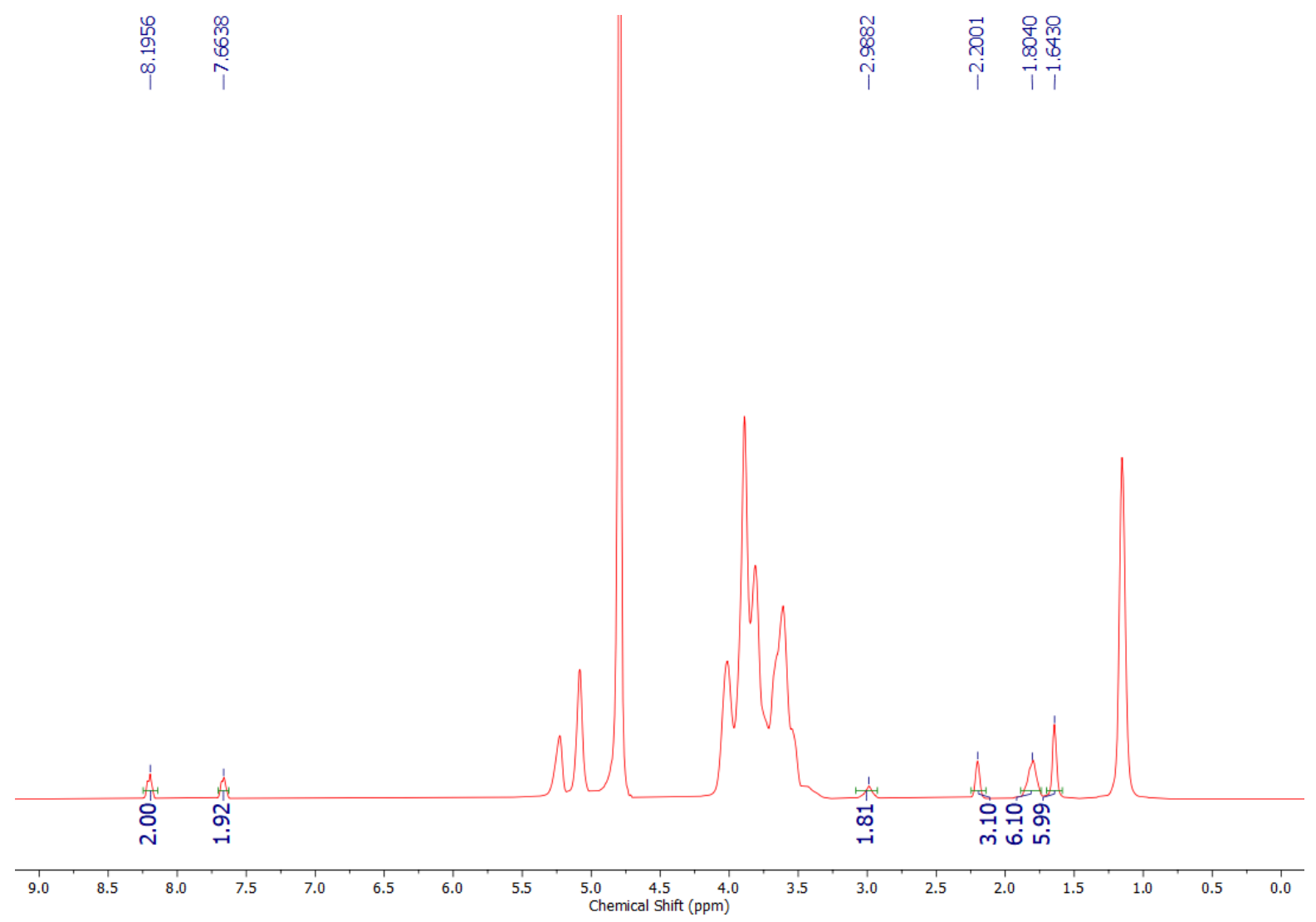

Figure S48. ${ }^{1} \mathrm{H}$ NMR $(400 \mathrm{MHz})$ spectrum of $\mathrm{HP}-\beta-\mathrm{CD} \cdot \mathbf{1}($ method 3$)$ in $\mathrm{D}_{2} \mathrm{O}$ at $298 \mathrm{~K}$. 


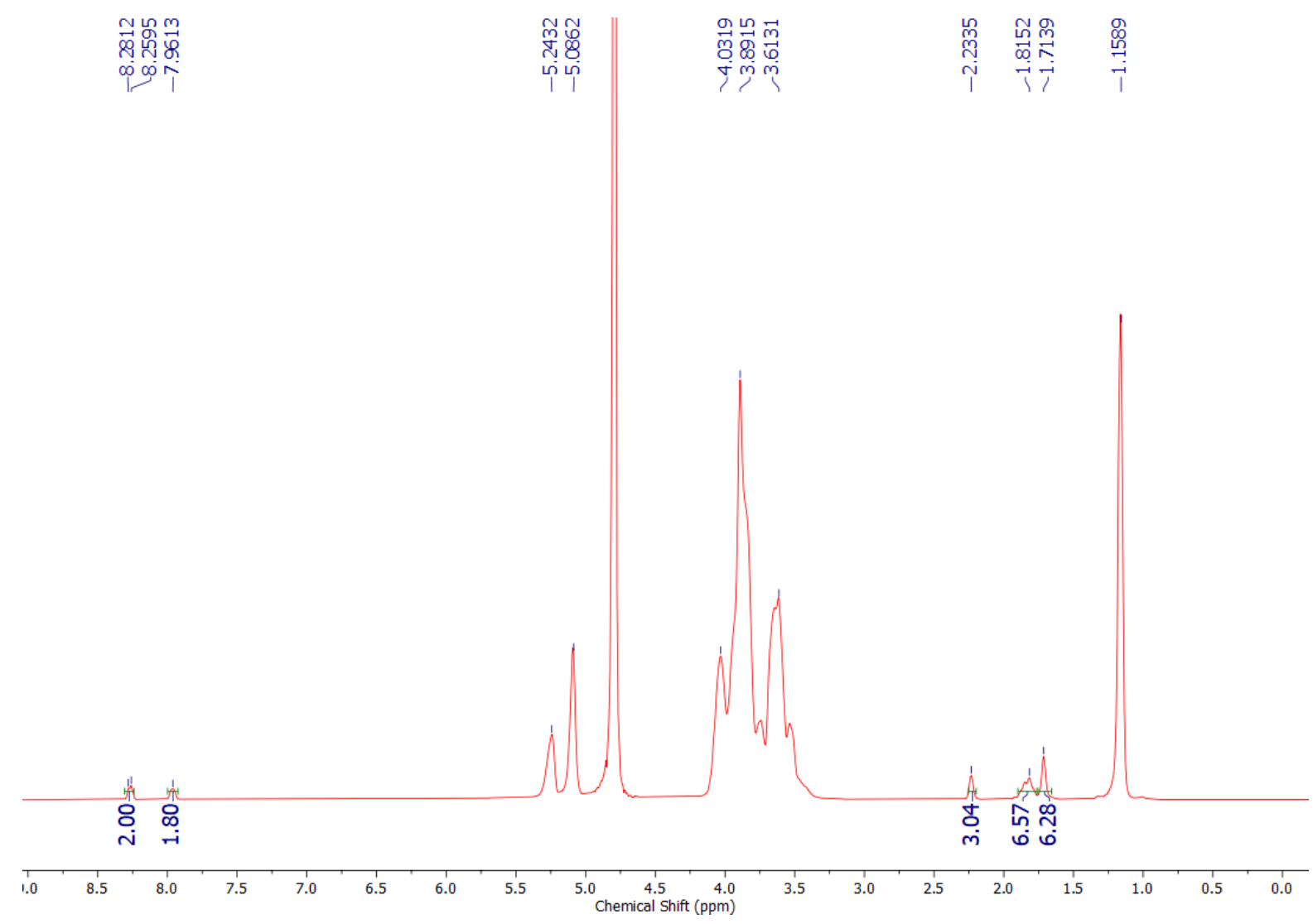

Figure S49. ${ }^{1} \mathrm{H}$ NMR $(400 \mathrm{MHz})$ spectrum of $\mathrm{HP}-\beta-\mathrm{CD} \cdot 2($ method 1$)$ in $\mathrm{D}_{2} \mathrm{O}$ at $298 \mathrm{~K}$.

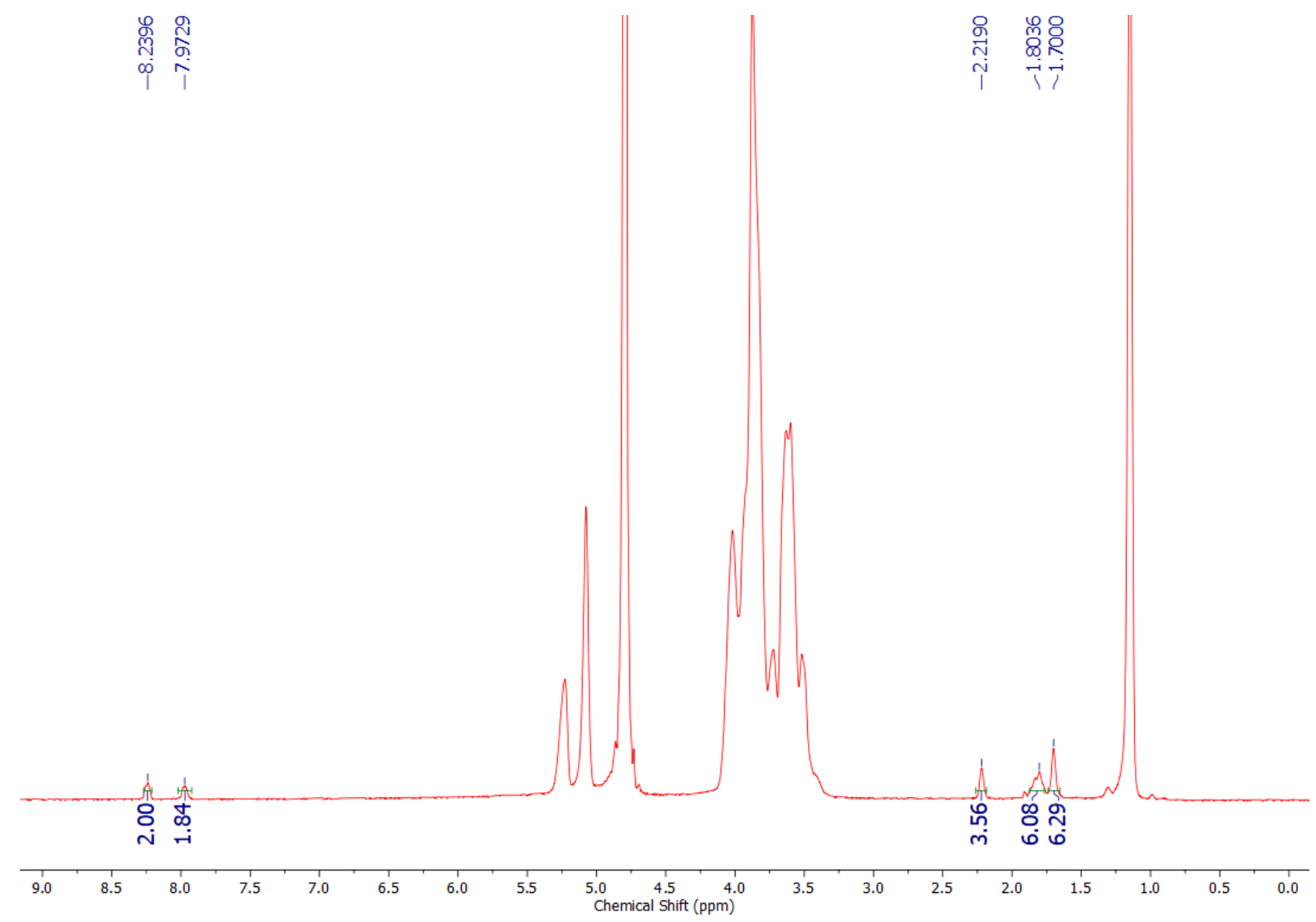

Figure S50. ${ }^{1} \mathrm{H}$ NMR (400 MHz) spectrum of HP- $\beta-C D \cdot 2($ method 2$)$ in $\mathrm{D}_{2} \mathrm{O}$ at $298 \mathrm{~K}$. 


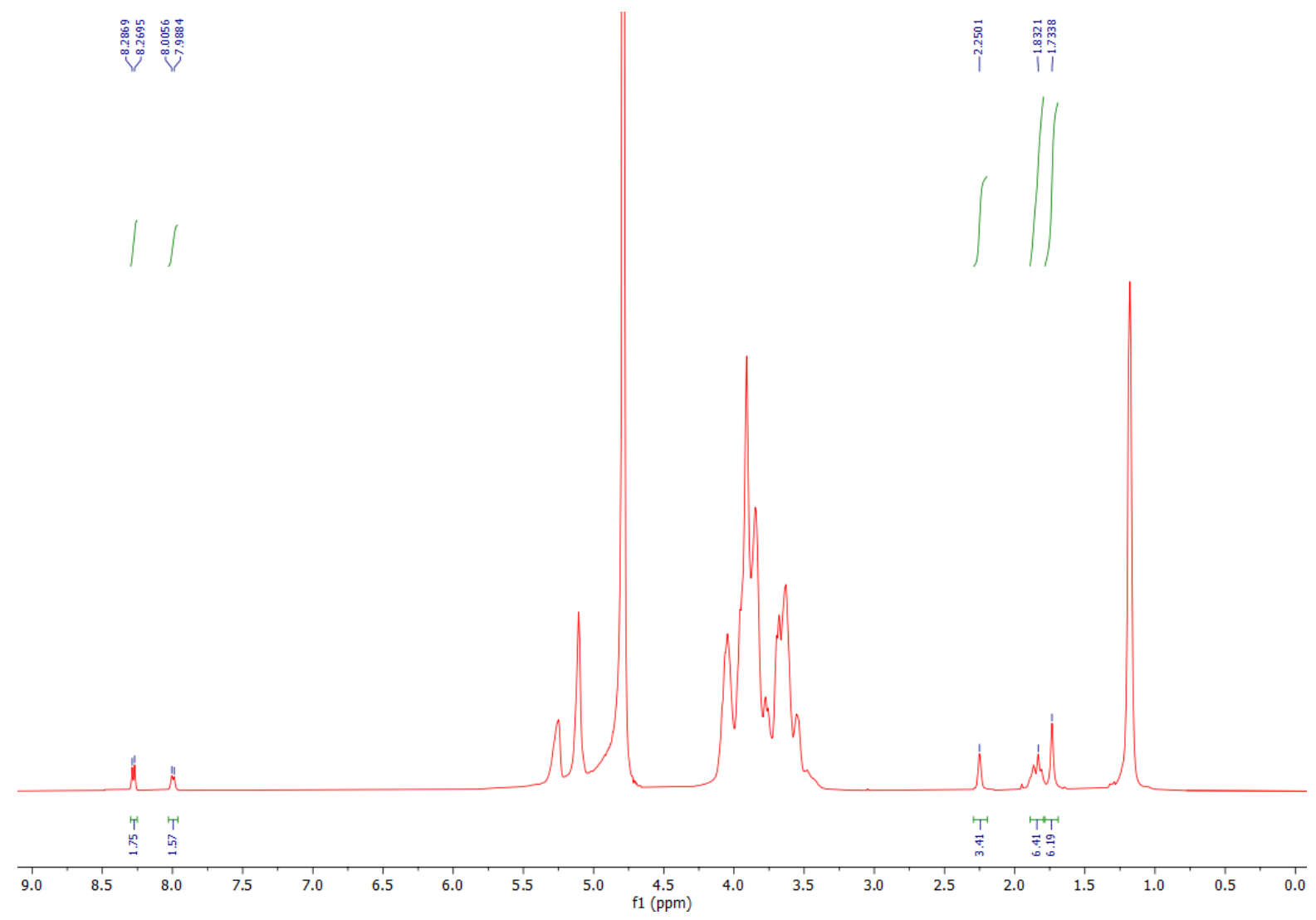

Figure S51. ${ }^{1} \mathrm{H}$ NMR $(400 \mathrm{MHz})$ spectrum of $\mathrm{HP}-\beta-\mathrm{CD} \cdot 2\left(\right.$ method 3) in $\mathrm{D}_{2} \mathrm{O}$ at $298 \mathrm{~K}$.

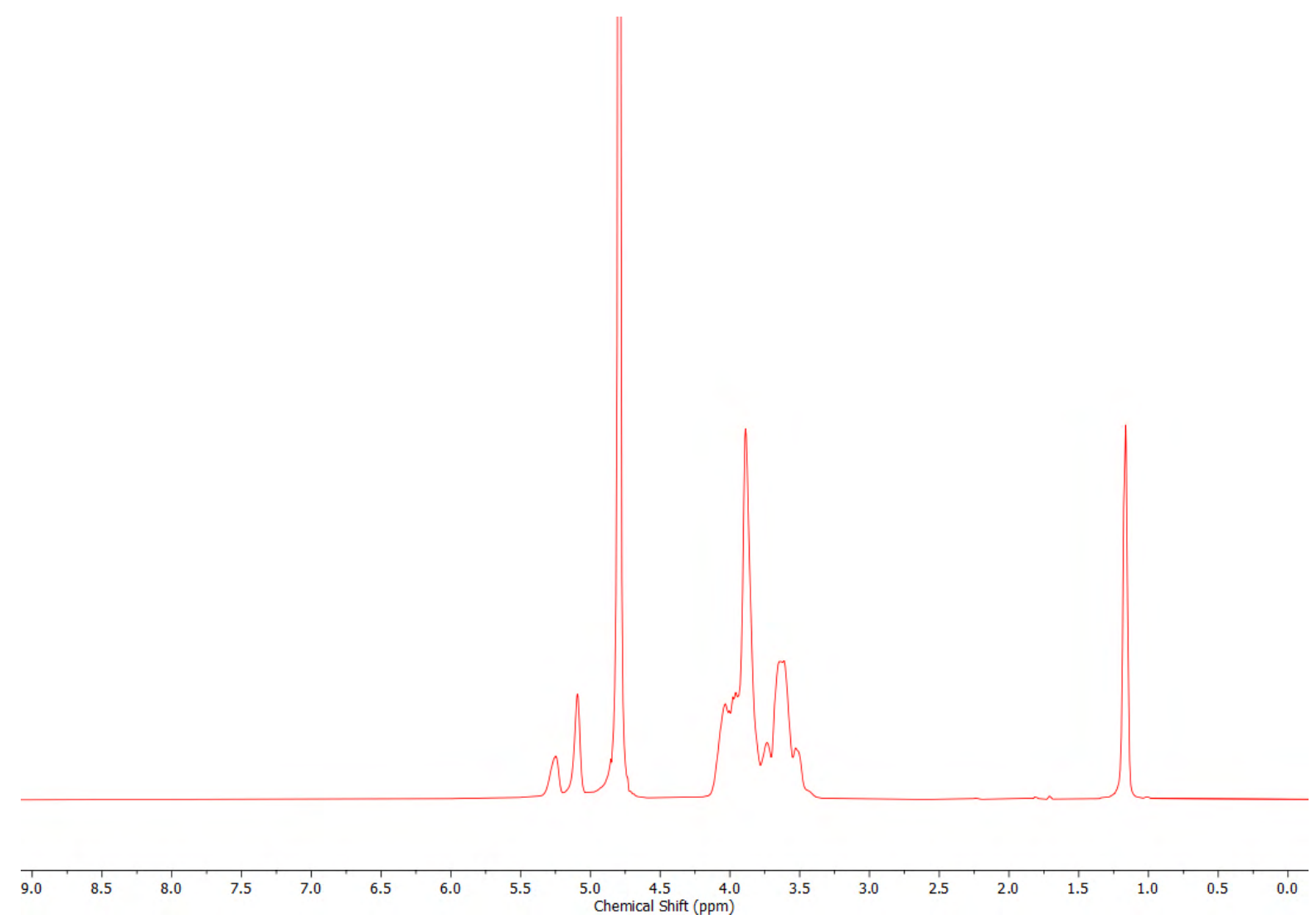

Figure S52. ${ }^{1} \mathrm{H}$ NMR (400 MHz) spectrum of $\mathrm{HP}-\beta-\mathrm{CD} \cdot 3$ (method 1$)$ in $\mathrm{D}_{2} \mathrm{O}$ at $298 \mathrm{~K}$. 


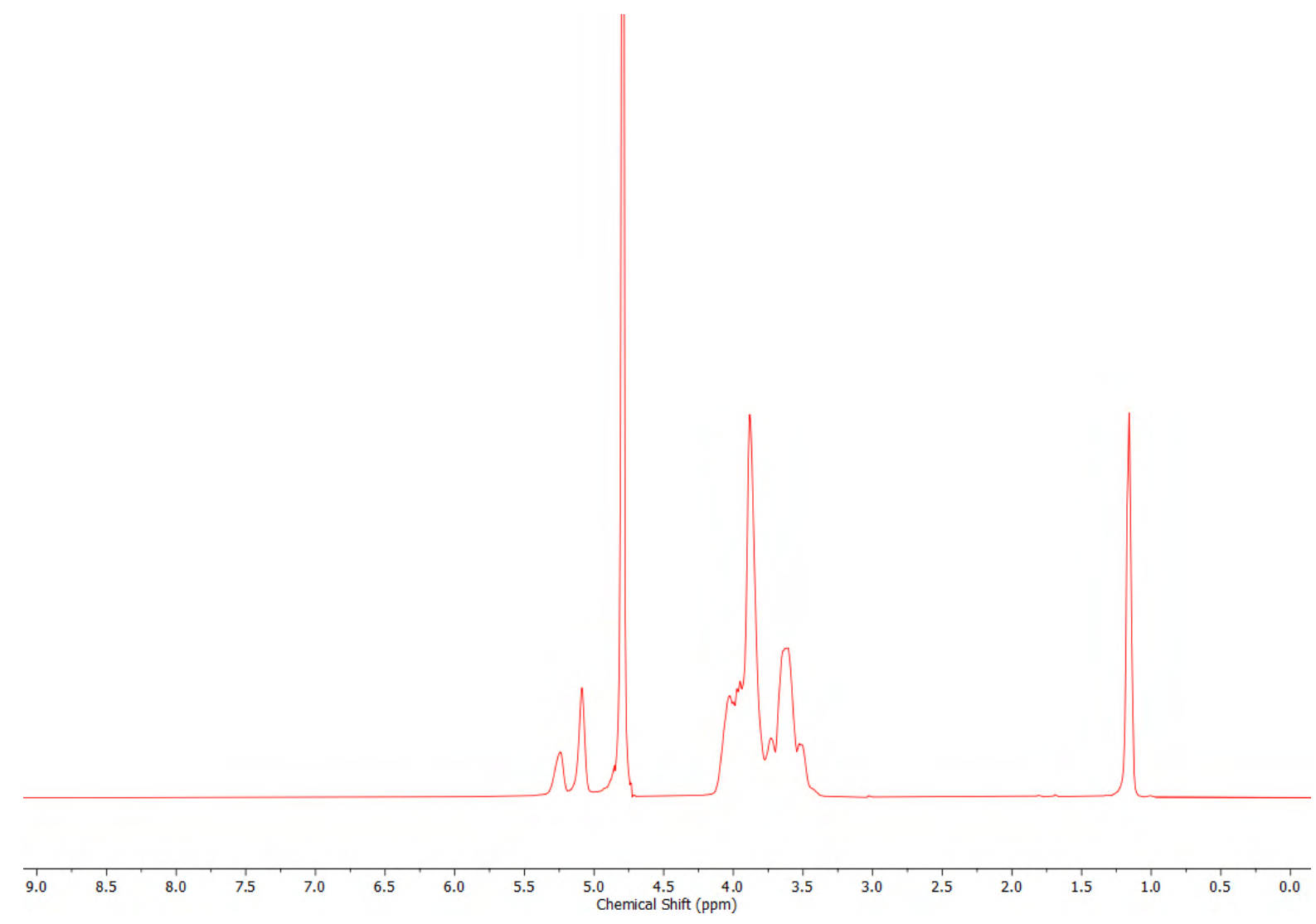

Figure S53. ${ }^{1} \mathrm{H}$ NMR $(400 \mathrm{MHz})$ spectrum of $\mathrm{HP}-\beta-\mathrm{CD} \cdot 3$ (method 2) in $\mathrm{D}_{2} \mathrm{O}$ at $298 \mathrm{~K}$.

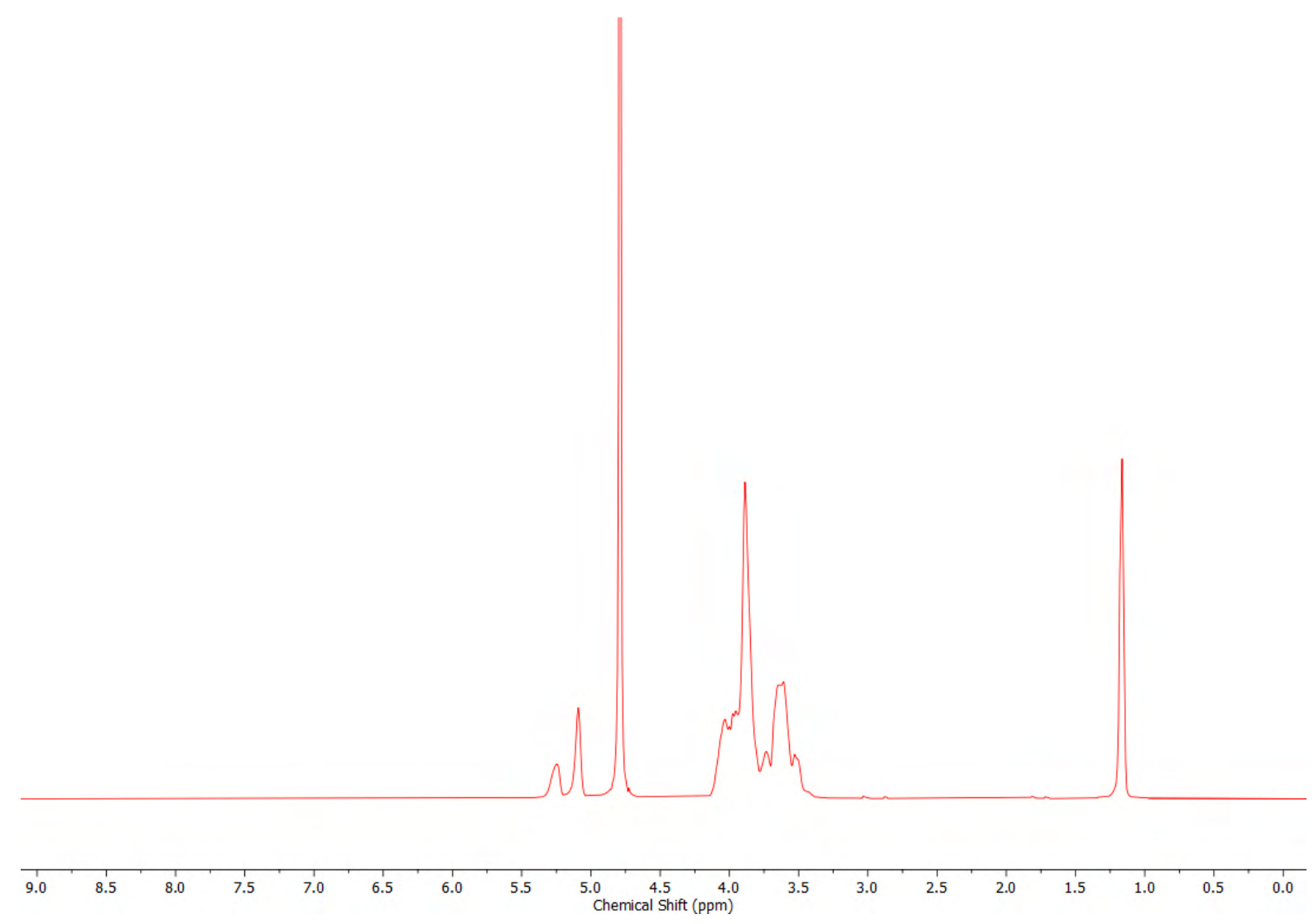

Figure S54. ${ }^{1} \mathrm{H}$ NMR (400 MHz) spectrum of HP- $\beta-C D \cdot 3\left(\right.$ method 3) in $\mathrm{D}_{2} \mathrm{O}$ at $298 \mathrm{~K}$. 


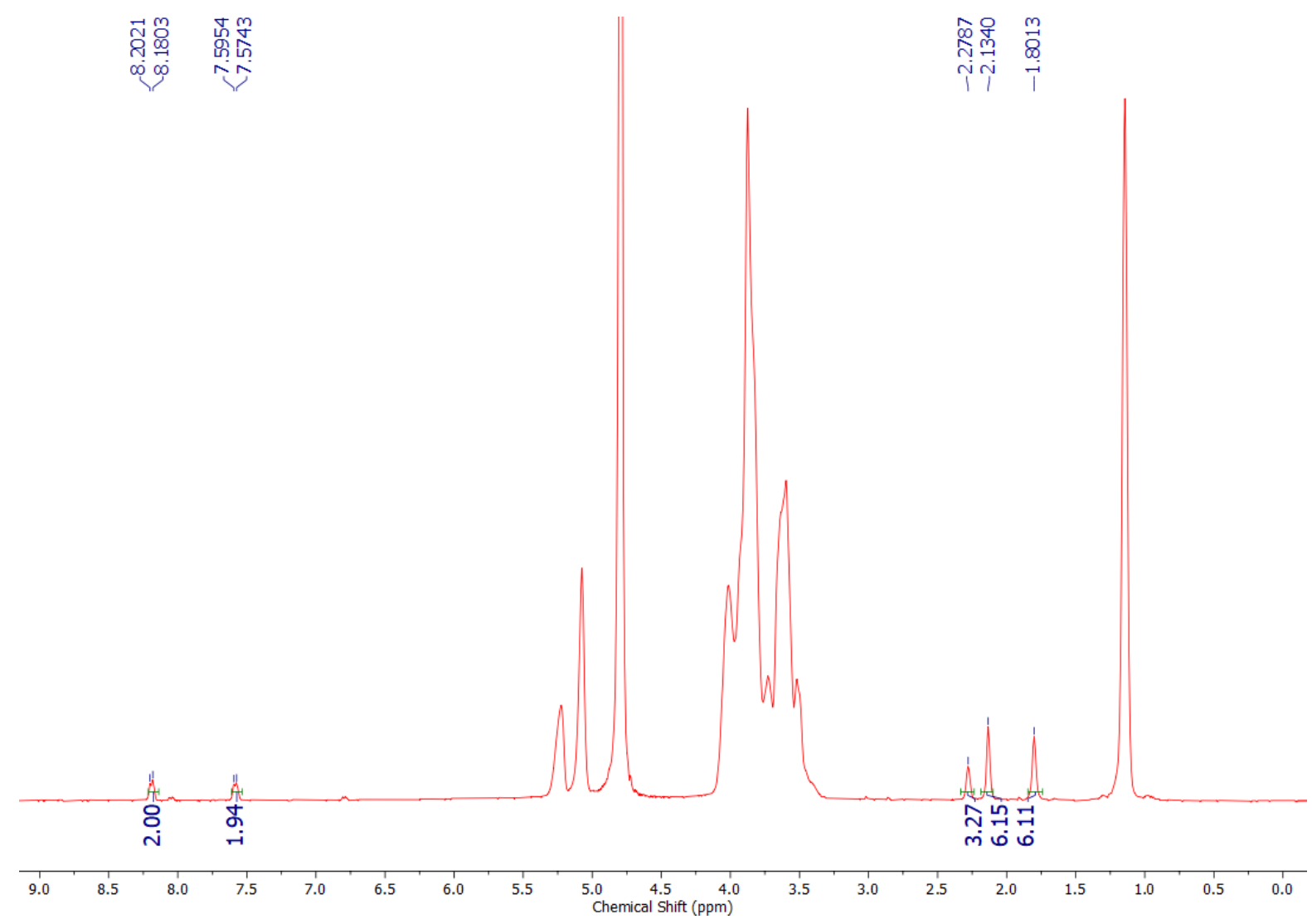

Figure S55. ${ }^{1} \mathrm{H}$ NMR $(400 \mathrm{MHz})$ spectrum of $\mathrm{HP}-\beta-\mathrm{CD} \cdot 4($ method 1$)$ in $\mathrm{D}_{2} \mathrm{O}$ at $298 \mathrm{~K}$.

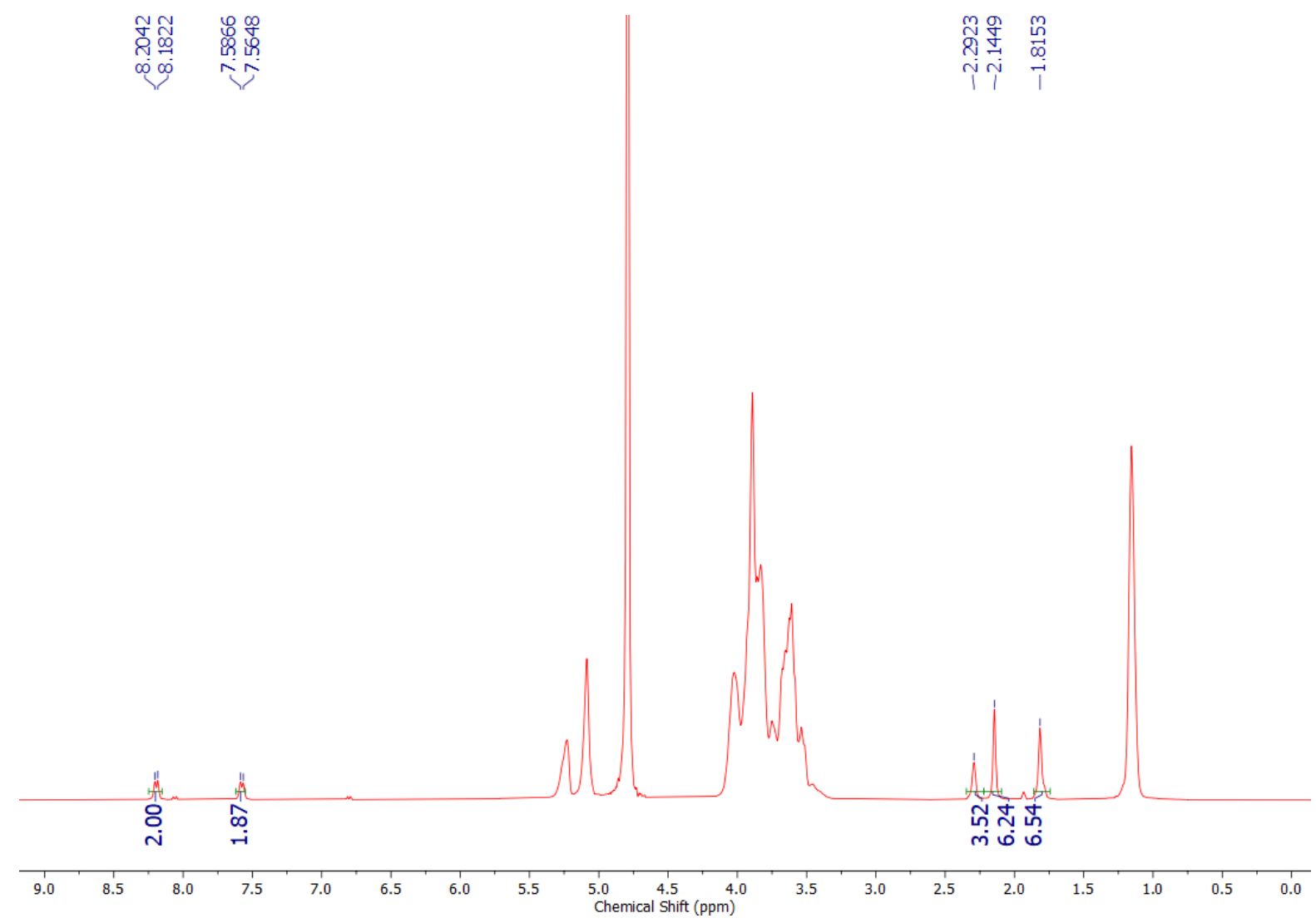

Figure S56. ${ }^{1} \mathrm{H}$ NMR (400 MHz) spectrum of $\mathrm{HP}-\beta-\mathrm{CD} \cdot 4$ (method 2$)$ in $\mathrm{D}_{2} \mathrm{O}$ at $298 \mathrm{~K}$. 


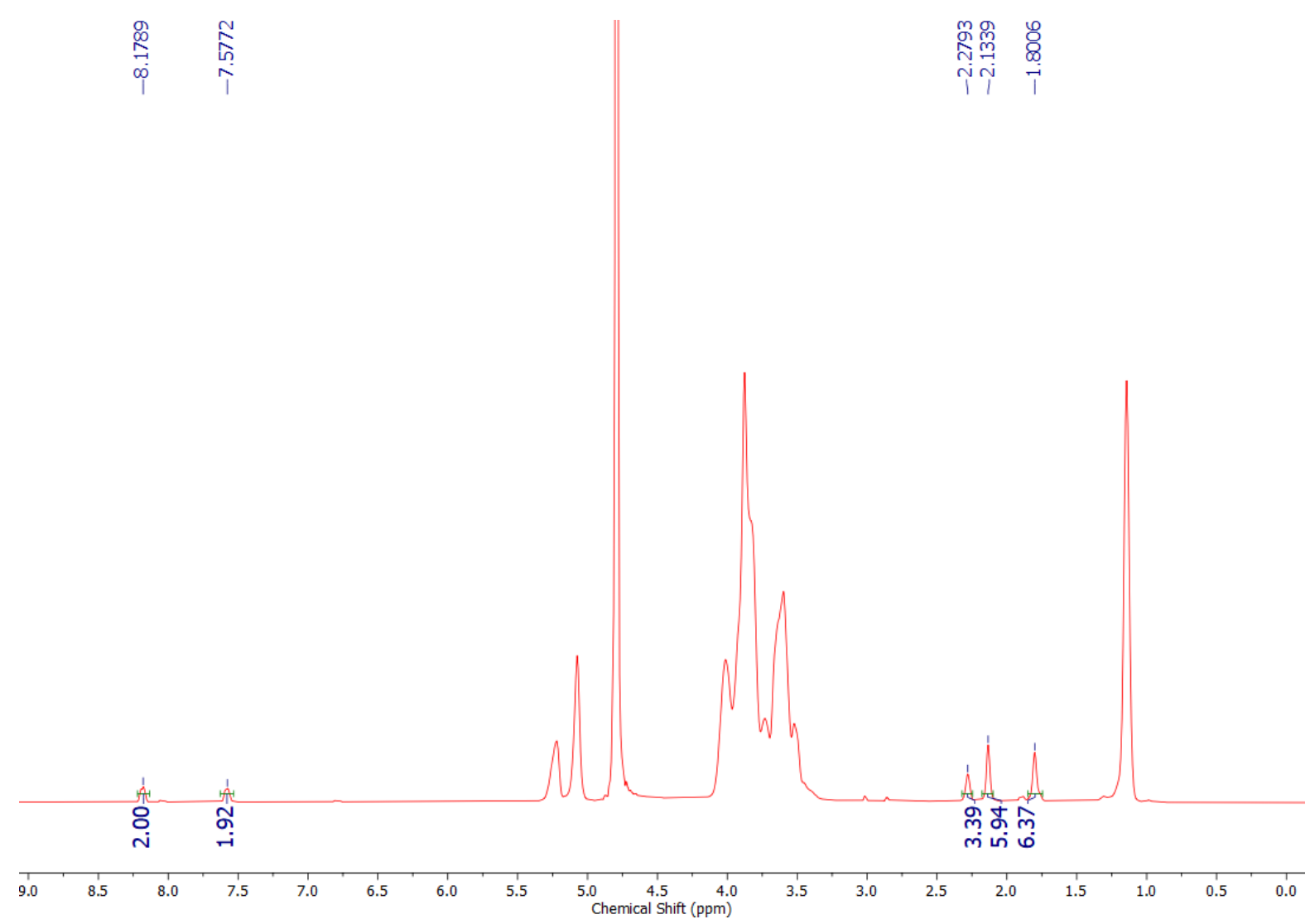

Figure S57. ${ }^{1} \mathrm{H}$ NMR (400 MHz) spectrum of $\mathrm{HP}-\beta-\mathrm{CD} \cdot 4\left(\right.$ method 3) in $\mathrm{D}_{2} \mathrm{O}$ at $298 \mathrm{~K}$.

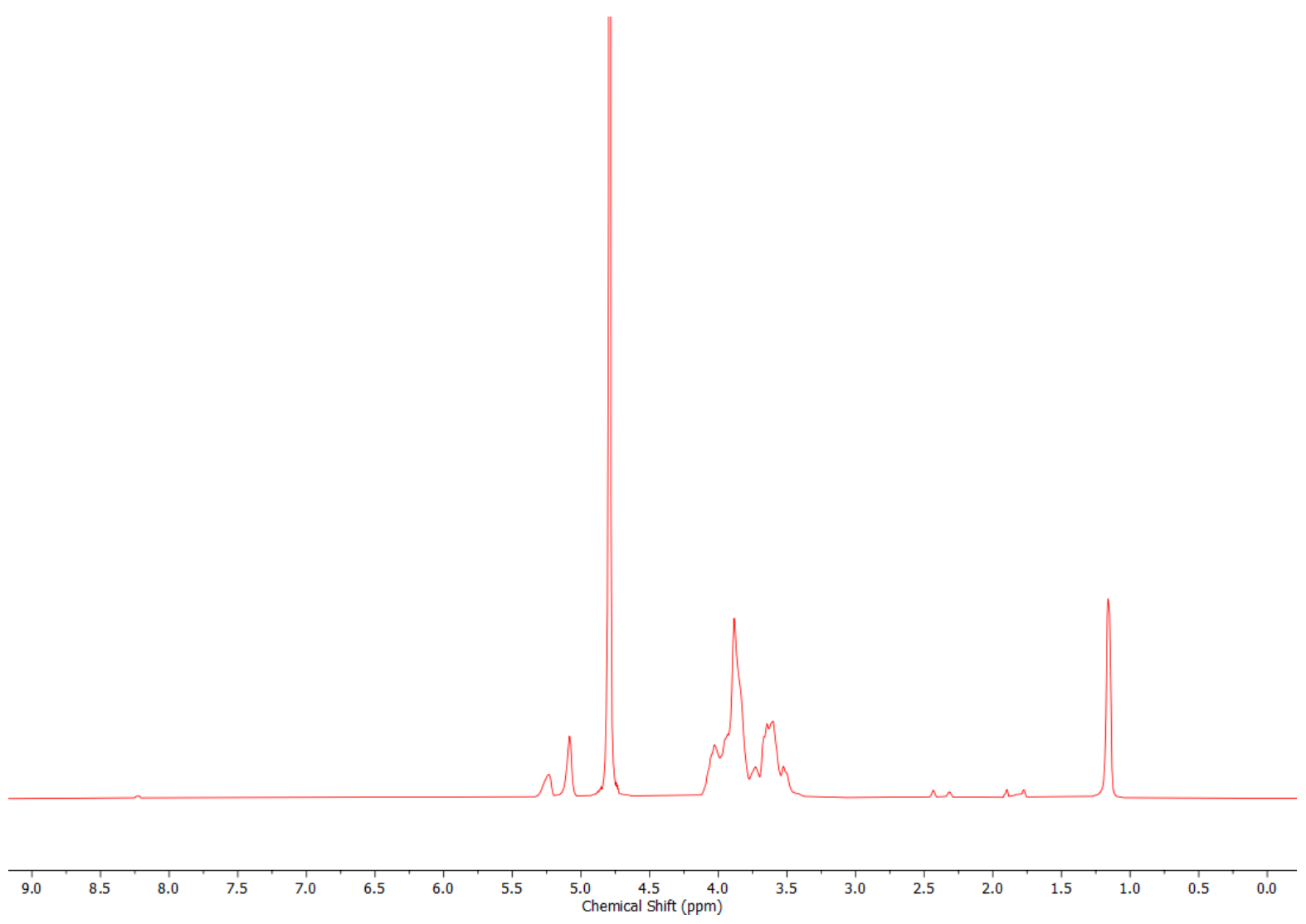

Figure S58. ${ }^{1} \mathrm{H}$ NMR (400 MHz) spectrum of HP- $\beta-C D \cdot 5$ (method 1$)$ in $\mathrm{D}_{2} \mathrm{O}$ at $298 \mathrm{~K}$. 


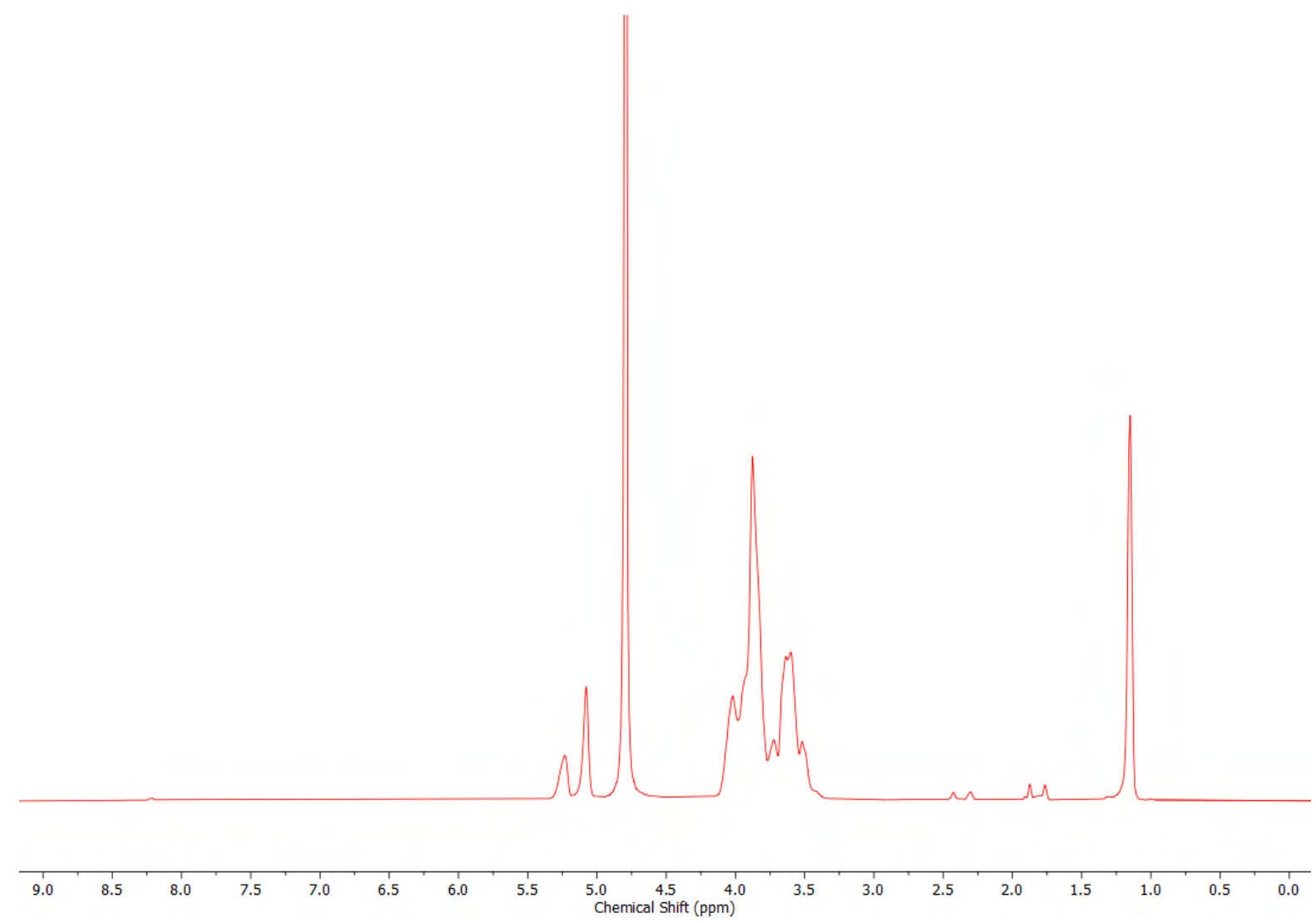

Figure S59. ${ }^{1} \mathrm{H}$ NMR (400 MHz) spectrum of HP- $\beta-C D \cdot 5$ (method 2) in $\mathrm{D}_{2} \mathrm{O}$ at $298 \mathrm{~K}$.

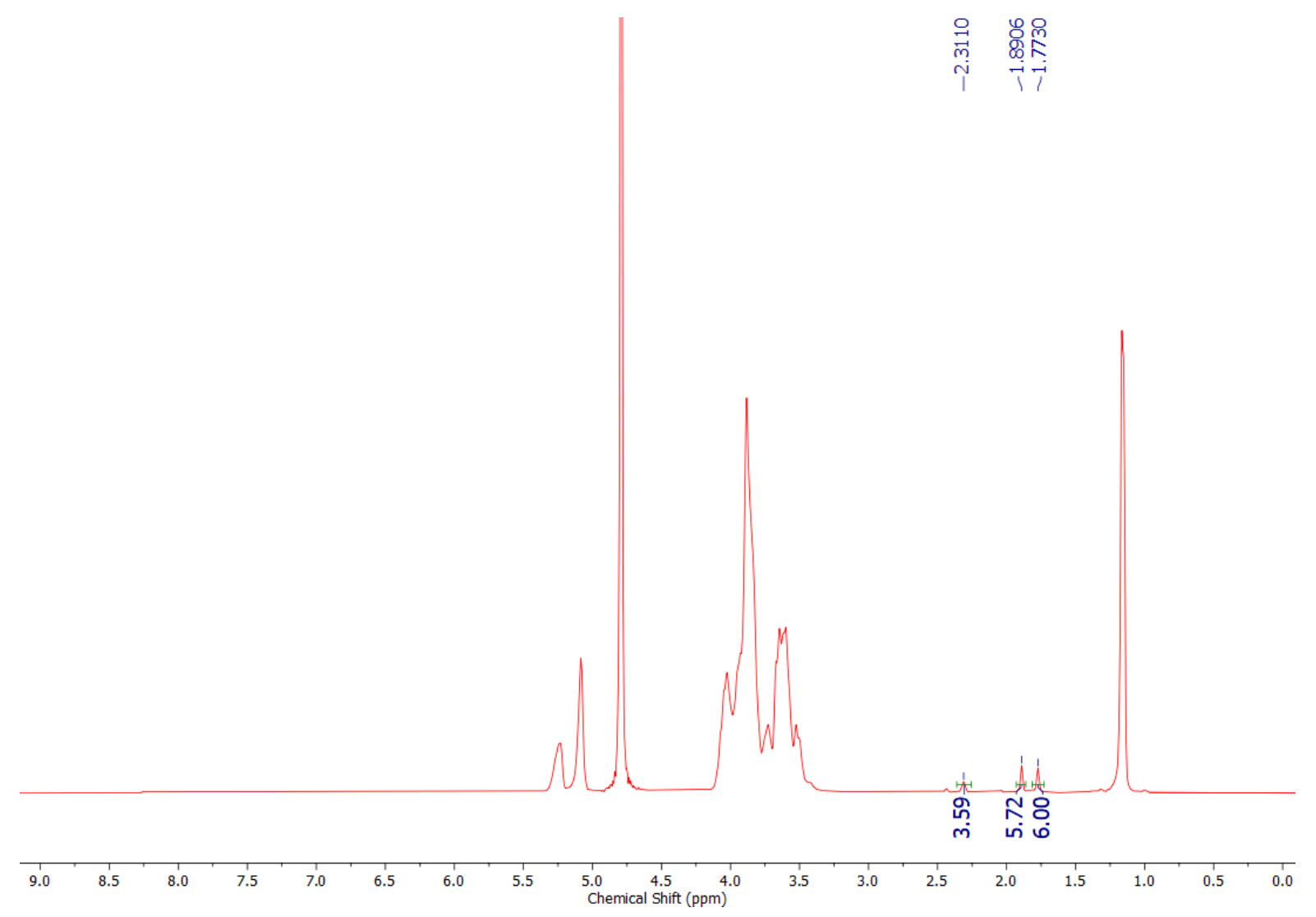

Figure S60. ${ }^{1} \mathrm{H}$ NMR (400 MHz) spectrum of HP- $\beta-C D \cdot 5\left(\right.$ method 3) in $\mathrm{D}_{2} \mathrm{O}$ at $298 \mathrm{~K}$. 


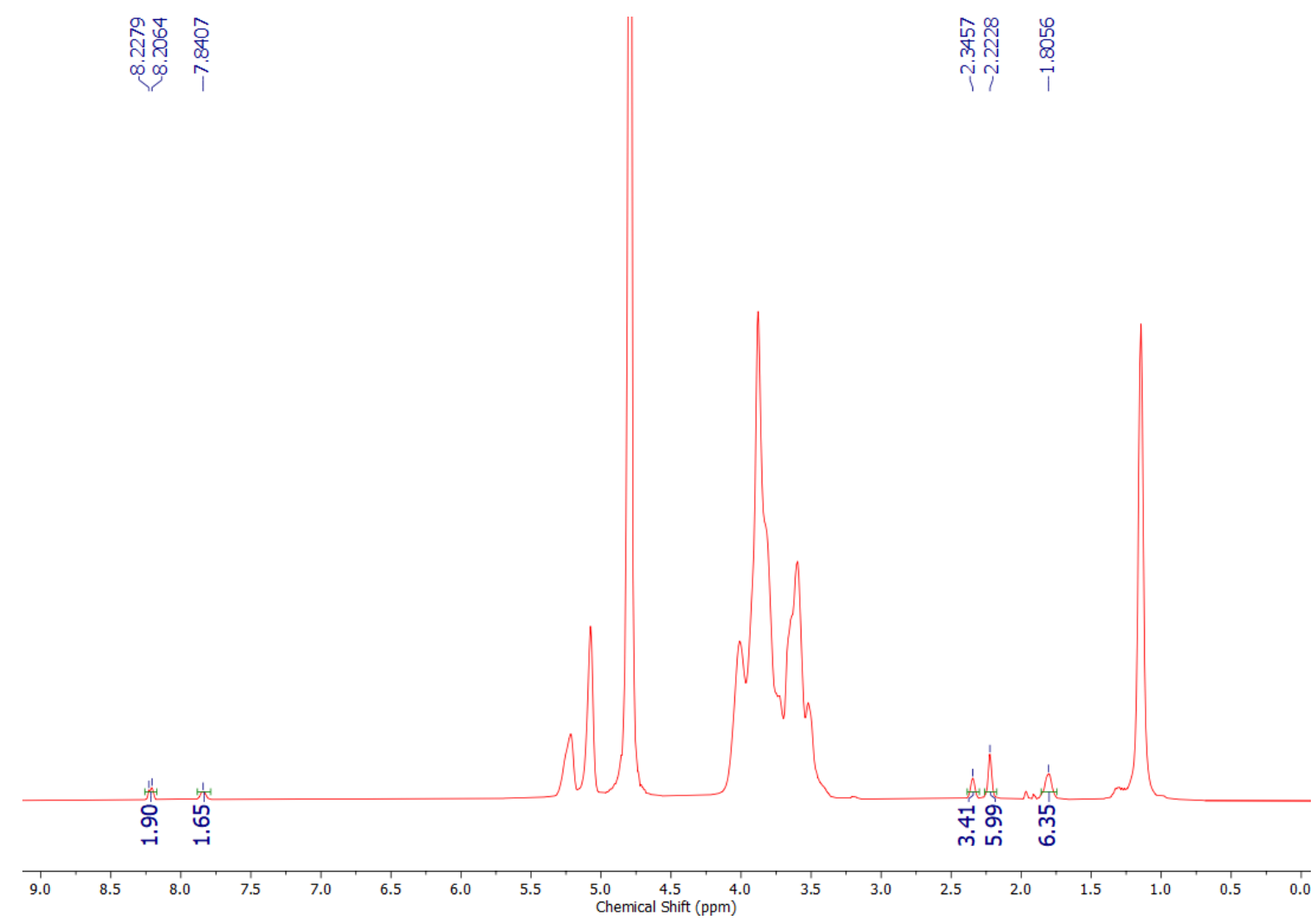

Figure S61. ${ }^{1} \mathrm{H}$ NMR (400 MHz) spectrum of HP- $\beta-C D \cdot 6$ (method 1) in $\mathrm{D}_{2} \mathrm{O}$ at $298 \mathrm{~K}$.

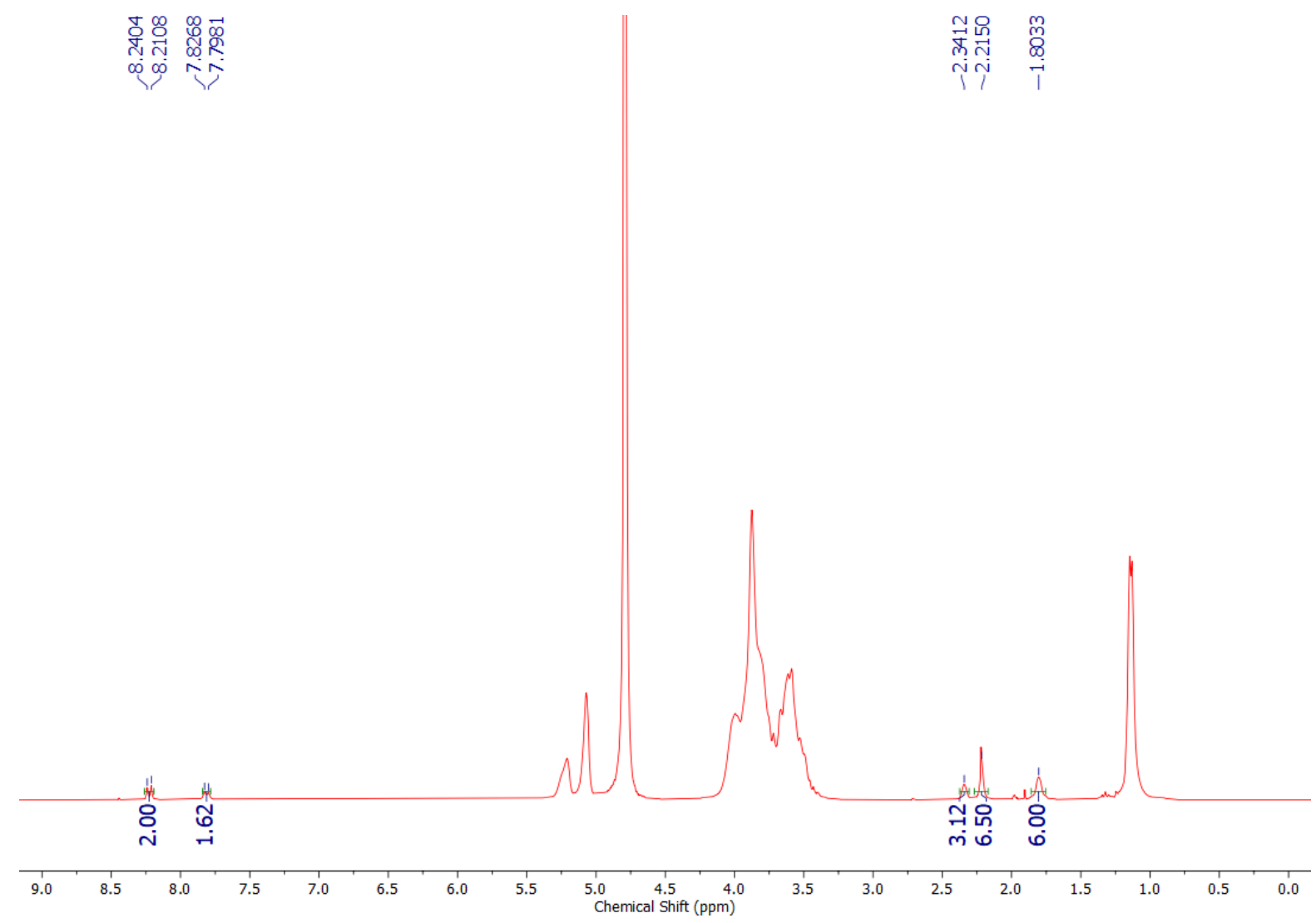

Figure S62. ${ }^{1} \mathrm{H}$ NMR $(400 \mathrm{MHz})$ spectrum of $\mathrm{HP}-\beta-\mathrm{CD} \cdot 6$ (method 2$)$ in $\mathrm{D}_{2} \mathrm{O}$ at $298 \mathrm{~K}$. 


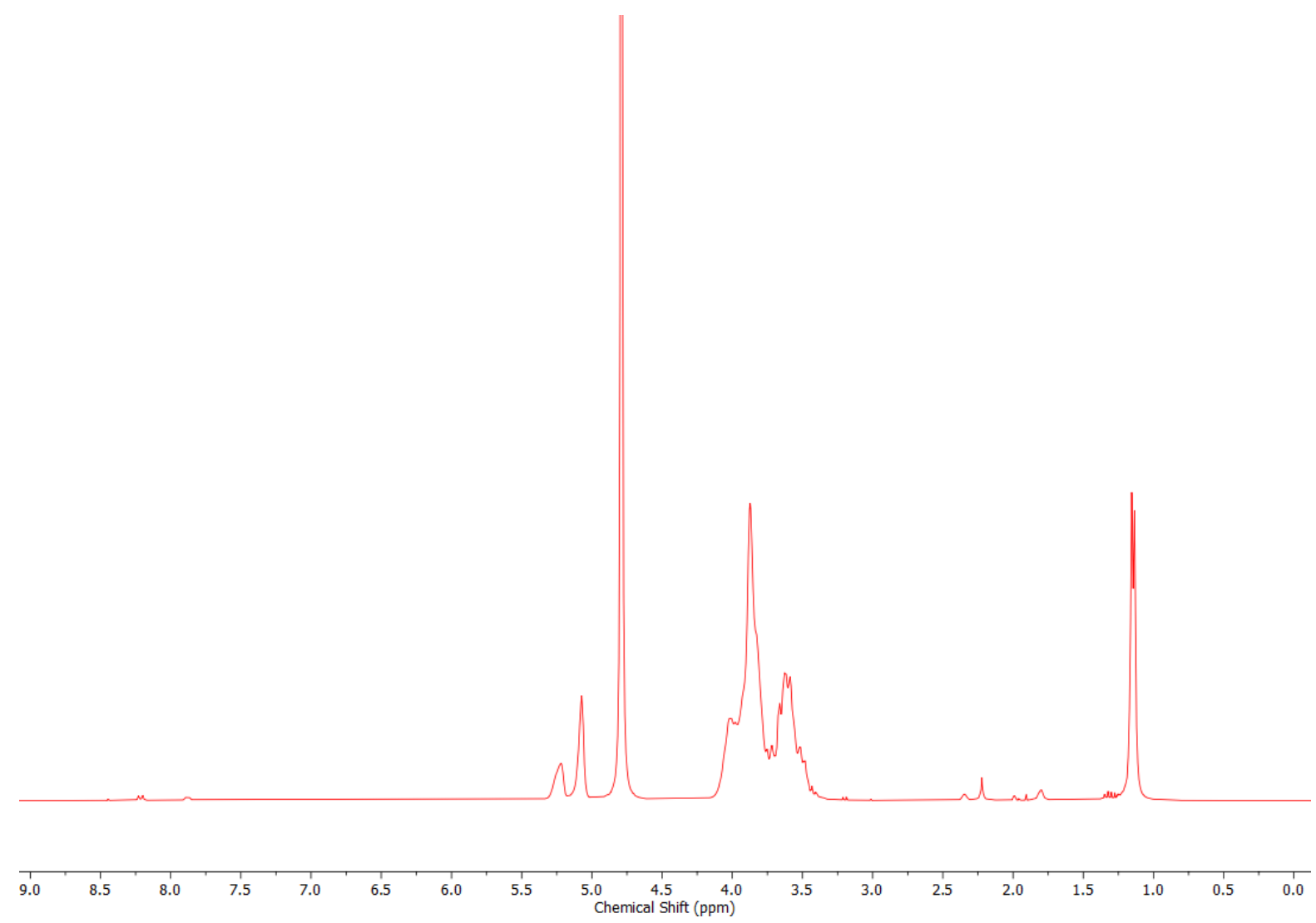

Figure S63. ${ }^{1} \mathrm{H}$ NMR $(400 \mathrm{MHz})$ spectrum of $\mathrm{HP}-\beta-\mathrm{CD} \cdot 6($ method 3$)$ in $\mathrm{D}_{2} \mathrm{O}$ at $298 \mathrm{~K}$. 


\section{UV-Vis Spectrometry}

\subsection{UV-Vis Calibration Spectra}

$\mathrm{UV}$-Vis studies in DMSO were performed to ascertain the percentage mass of transporter present in each inclusion complex. DMSO was used ahead of $\mathrm{H}_{2} \mathrm{O}$ as the free transporters 1-6 readily dissolve in this medium, and the solvent possesses a relatively low UV wavelength cut-off. ${ }^{15}$ The UV cut-off is the wavelength at which the solvent absorbance in a $1 \mathrm{~cm}$ path length equals 1 absorbance unit $(A)$, and absorbance becomes excessive at wavelengths below this value. UV-vis spectra were initially collected at five concentrations ranging from 5-50 $\mu \mathrm{M}$ for the free compounds 1-6. Plotting concentration against the maximum absorbance returned a linear relationship in accord with the Beer-Lambert Law. This is given in Equation S1:

$$
A=\varepsilon c l
$$

Equation S1. The Beer-Lambert Law

Where; $A$ is absorbance, $c$ is the concentration of the species, $l$ is the path length in $\mathrm{cm}$ (for these experiments, $l=1 \mathrm{~cm}$ ), and $\varepsilon$ is the molar extinction coefficient. The gradient of the linear fit calculated for the plot of $A$ against $c$ can be taken as equal to $\varepsilon$.

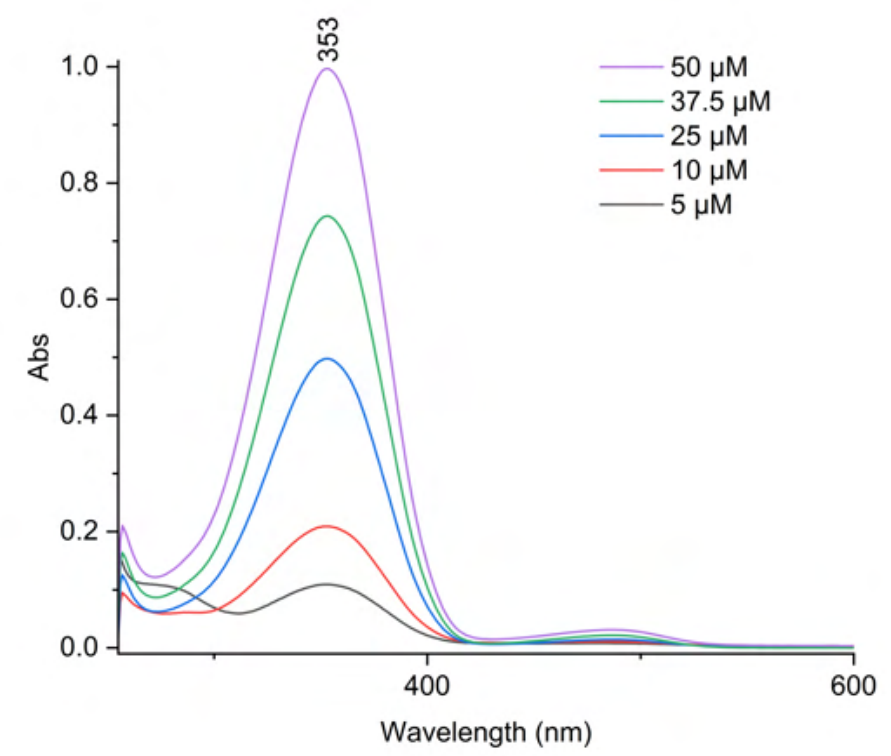

Figure S64. The UV-Vis calibration traces of 1 , for concentrations ranging from 5-50 $\mu \mathrm{M}$. The wavelength of maximum absorbance is labelled (353 nm). 


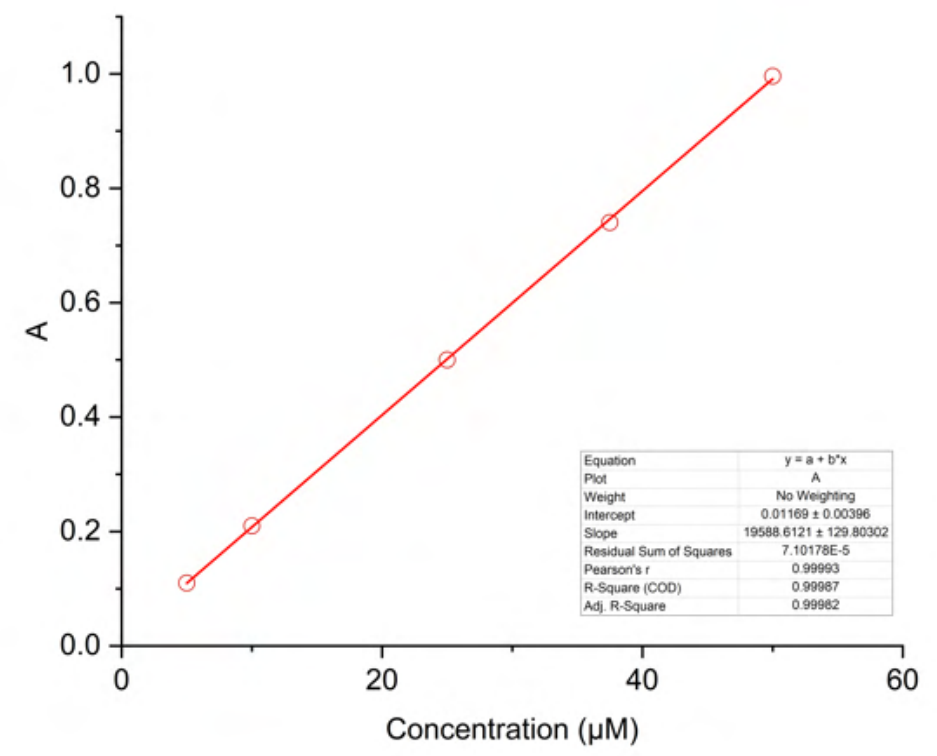

Figure S65. Plot of maximum absorbance against concentration for $\mathbf{1}$. The gradient provides a molar extinction coefficient value of $\varepsilon=19589 \mathrm{M}^{-1} \mathrm{~cm}^{-1}$.

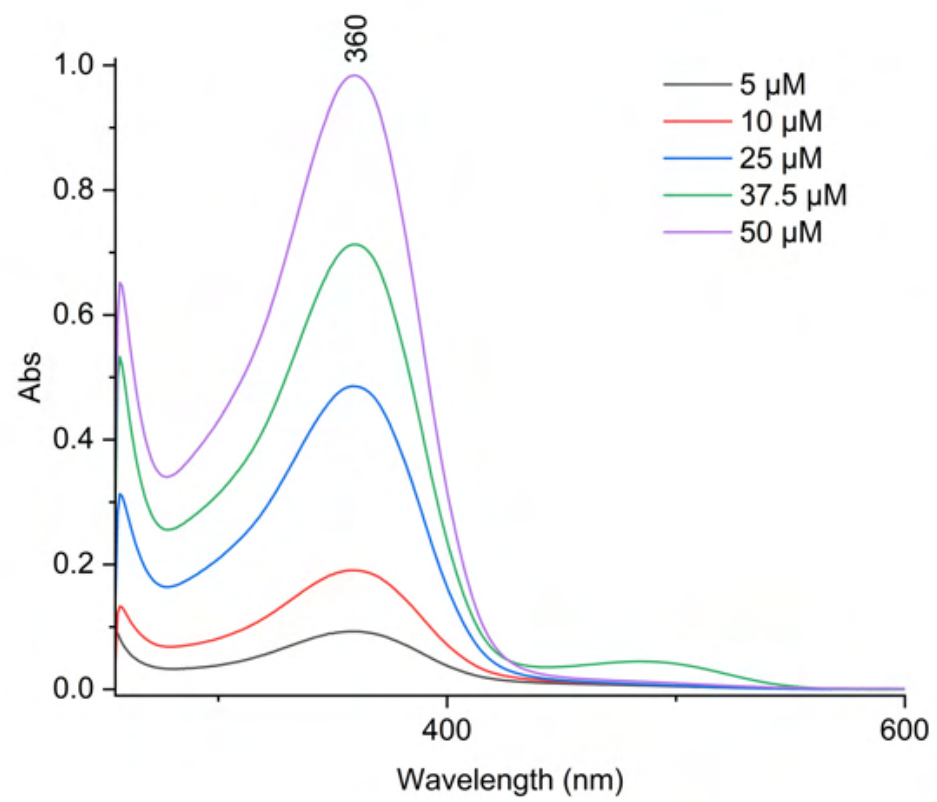

Figure S66. The UV-Vis calibration traces of 2 , for concentrations ranging from 5-50 $\mu \mathrm{M}$. The wavelength of maximum absorbance is labelled $(360 \mathrm{~nm})$. 


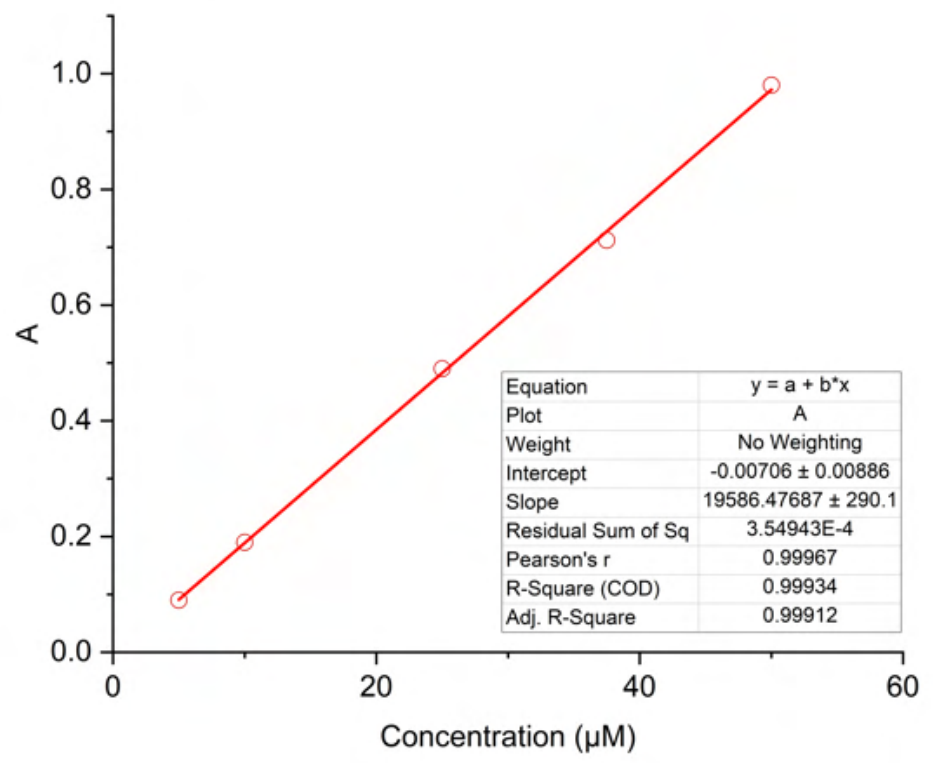

Figure S67. Plot of maximum absorbance against concentration for 2 . The gradient provides a molar extinction coefficient value of $\varepsilon=19586 \mathrm{M}^{-1} \mathrm{~cm}^{-1}$.

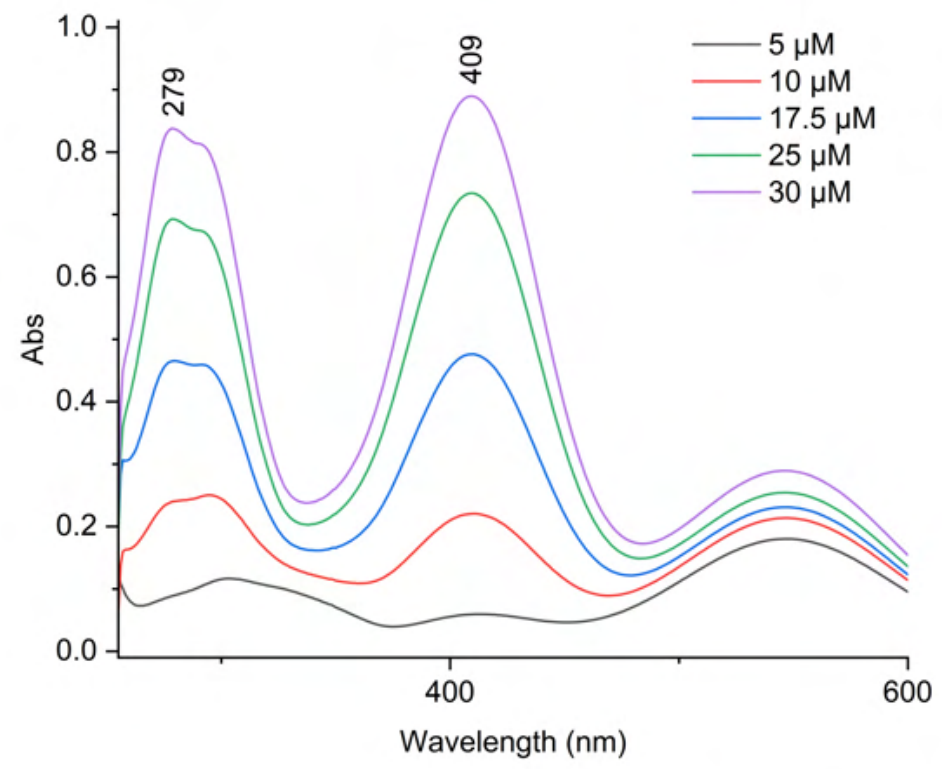

Figure S68. The UV-Vis calibration traces of 3, for concentrations ranging from 5-30 $\mu \mathrm{M}$. The wavelength of maximum absorbance is labelled (409 nm). 


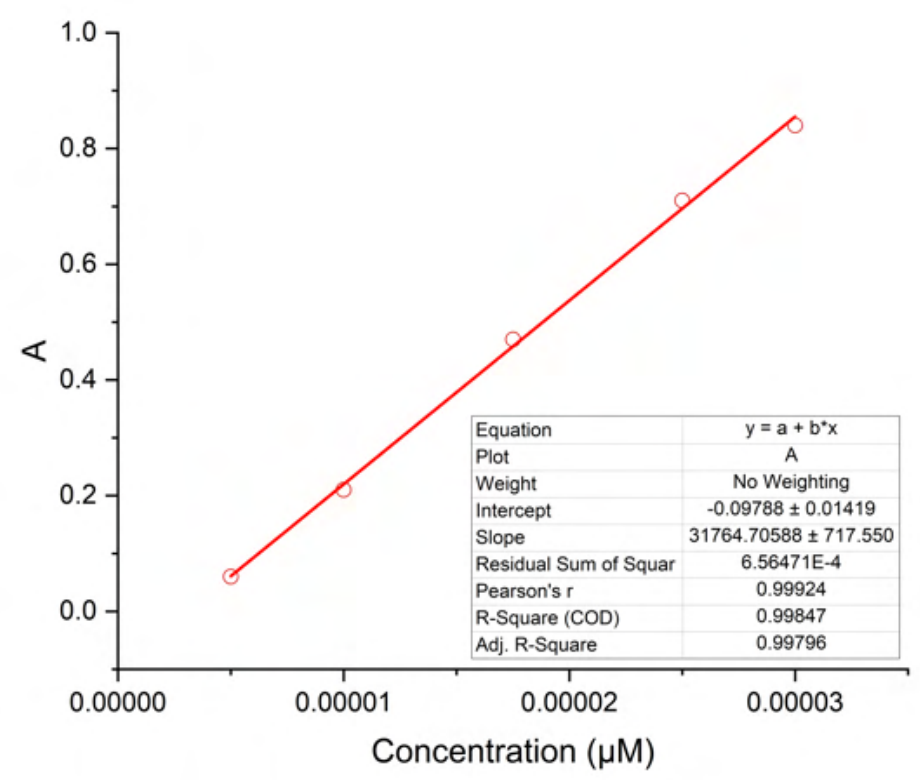

Figure S69. Plot of maximum absorbance against concentration for 3 . The gradient provides a molar extinction coefficient value of $\varepsilon=33412 \mathrm{M}^{-1} \mathrm{~cm}^{-1}$.

The small quantities of $\mathbf{3}$ present in the inclusion complex mixtures for this compound meant that calibration quantification was not repeated in the presence of perchloric acid (explained below).

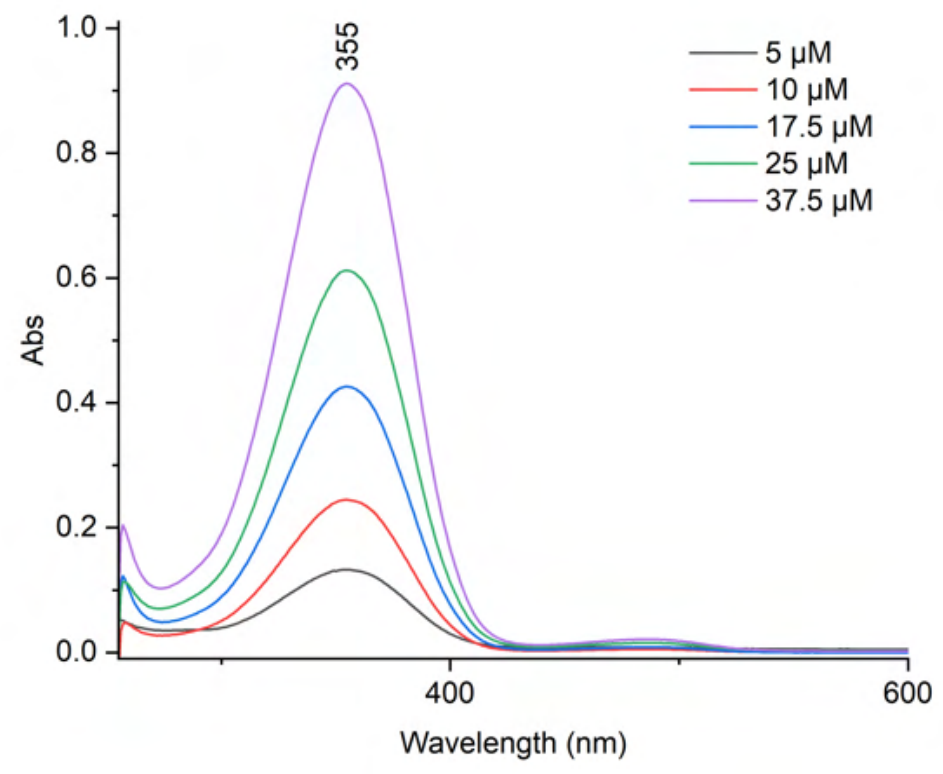

Figure S70. The UV-Vis calibration traces of 4 , for concentrations ranging from $5-37.5 \mu \mathrm{M}$. The wavelength of maximum absorbance is labelled $(355 \mathrm{~nm})$. 


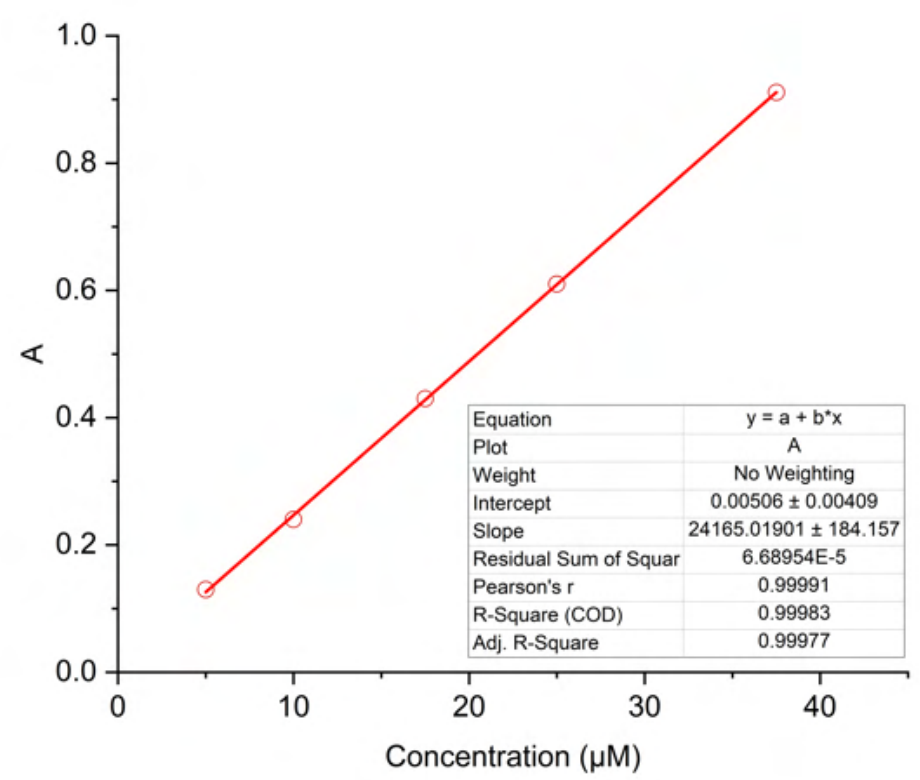

Figure S71. Plot of maximum absorbance against concentration for 4 . The gradient provides a molar extinction coefficient value of $\varepsilon=24165 \mathrm{M}^{-1} \mathrm{~cm}^{-1}$.

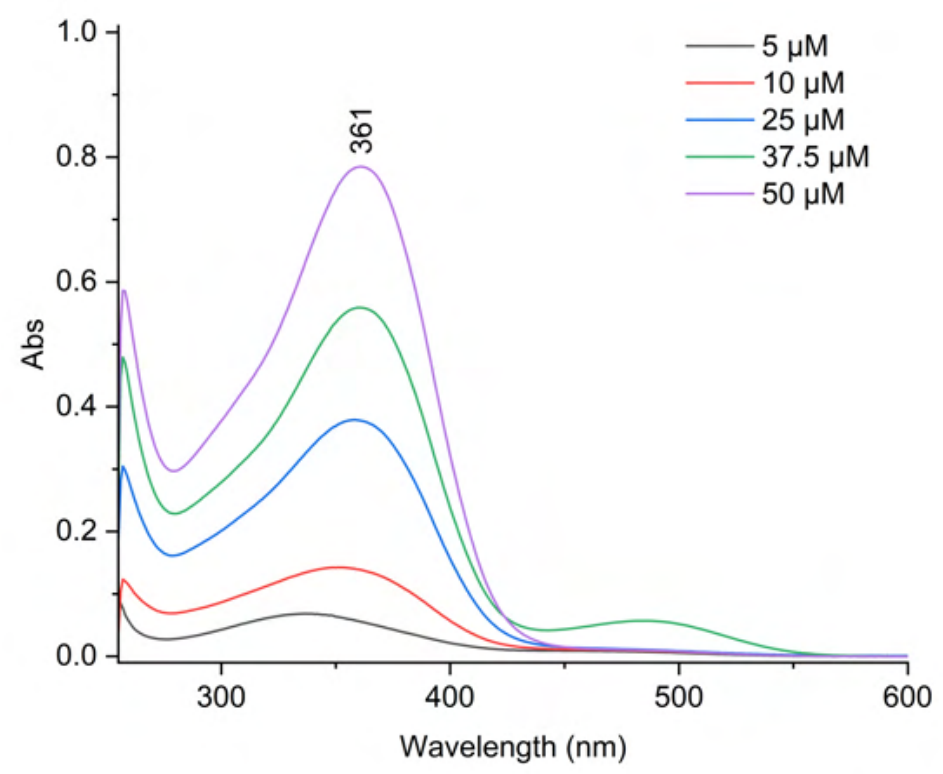

Figure S72. The UV-Vis calibration traces of 5, for concentrations ranging from 5-50 $\mu \mathrm{M}$. The wavelength of maximum absorbance is labelled $(361 \mathrm{~nm})$. 


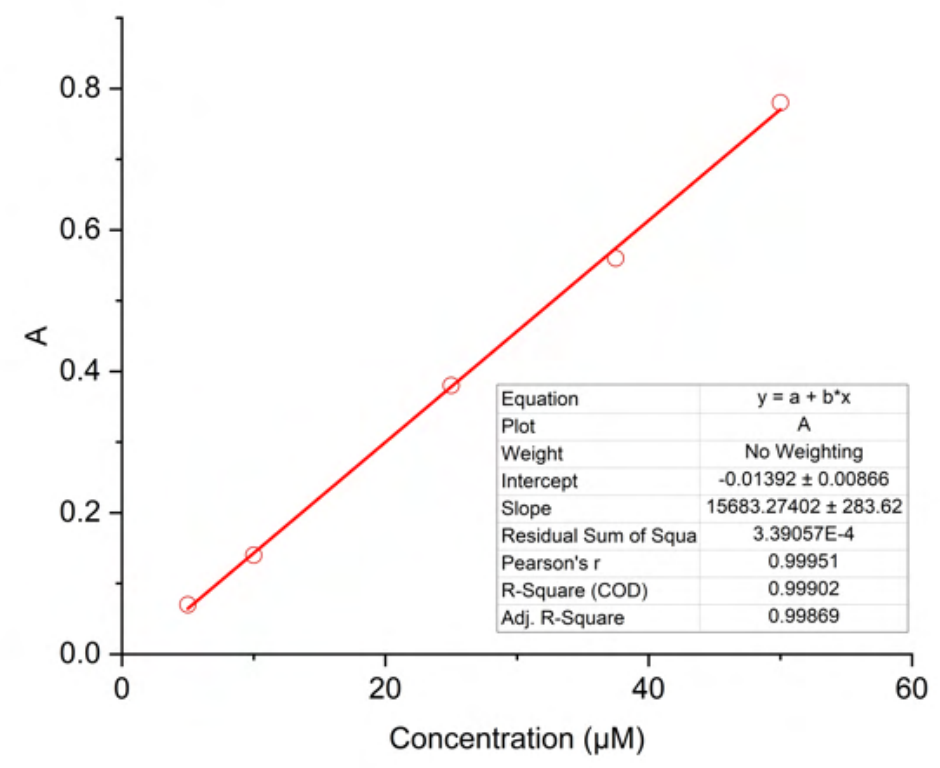

Figure S73. Plot of maximum absorbance against concentration for $\mathbf{5}$. The gradient provides a molar extinction coefficient value of $\varepsilon=15683 \mathrm{M}^{-1} \mathrm{~cm}^{-1}$.

An interesting property of squaramide receptors 3 and 6 was encountered during the serial dilution and initial UV-Vis calibration experiments of these compounds. Both compounds were a dark yellow colour at a concentration of $200 \mu \mathrm{M}$ in DMSO. This colour faded as the concentration decreased until around $50 \mu \mathrm{M}$, where a new hue began to appear. At a concentration of $5 \mu \mathrm{M}$, the solutions were a vibrant purple in colour (Figure S74). The photophysical behaviour underpinning this colour change became apparent when examining the UV-Vis calibration spectra for $\mathbf{6}$. The absorbance peaks at wavelengths 283,293 , and 408 $\mathrm{nm}$ decreased linearly with concentration, whereas the peak at $539 \mathrm{~nm}$ decreased in a nonlinear fashion. In the UV-Vis spectrum for 6 at $5 \mu \mathrm{M}$, the peak at $539 \mathrm{~nm}$ was the maximum absorbance of the system.

A similar phenomenon was reported by Rostami et al. during their investigations into $N, N^{\prime}-$ diarylsquaramides also containing a $p$-nitrophenyl derivative. ${ }^{16}$ Due to the high polarity of DMSO, deprotonation of one of the squaramide $\mathrm{N}-\mathrm{H}$ groups occurs at low concentrations without the addition of a base. Whereas at high concentrations, intermolecular hydrogen bonding between the $\mathrm{N}-\mathrm{H}$ groups and the dicarbonyl moieties of other squaramide molecules limited the level of deprotonation. Dilution caused the molecules to be more dispersed in solution and reduced the self-association of the molecules. Less intermolecular hydrogen 
bonds cause deprotonation to occur more regularly, resulting in the observed colour change from yellow to purple. To circumvent possible deprotonation, a small aliquot of perchloric acid $(0.01 \mathrm{M})$ was added to the solutions 6 used in UV-Vis experiments. Perchloric acid does not interfere with the UV-Vis trace and ensures the compounds do not deprotonate. The amount of transporter present in the inclusion complex mixtures of $\mathbf{3}$ did not justify the repetition of experiments in the presence of perchloric acid.

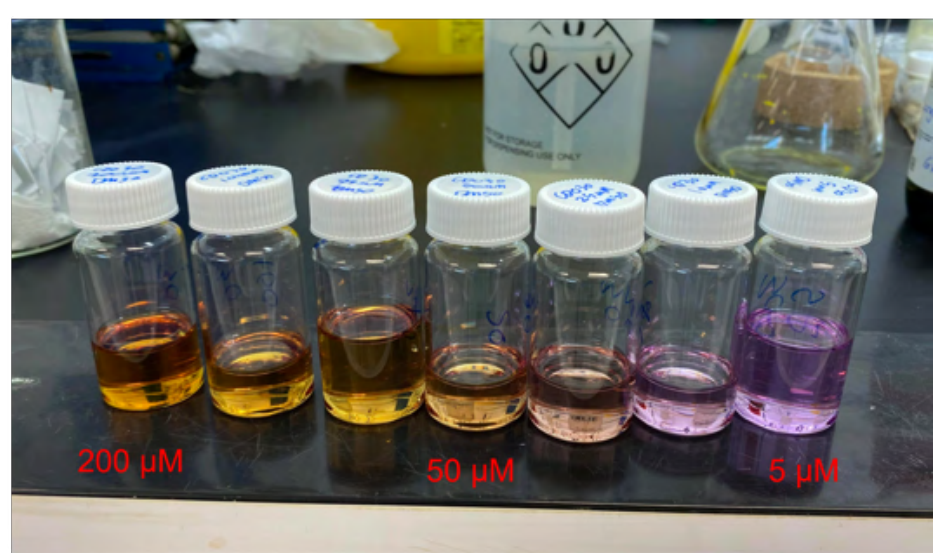

Figure S74. The colour change from orange to purple occurred during the serial dilution of compound 6 due to the deprotonation of the receptor at low concentrations. ${ }^{16}$ The problem was circumvented with the addition of an aliquot of perchloric acid (0.01 M). 


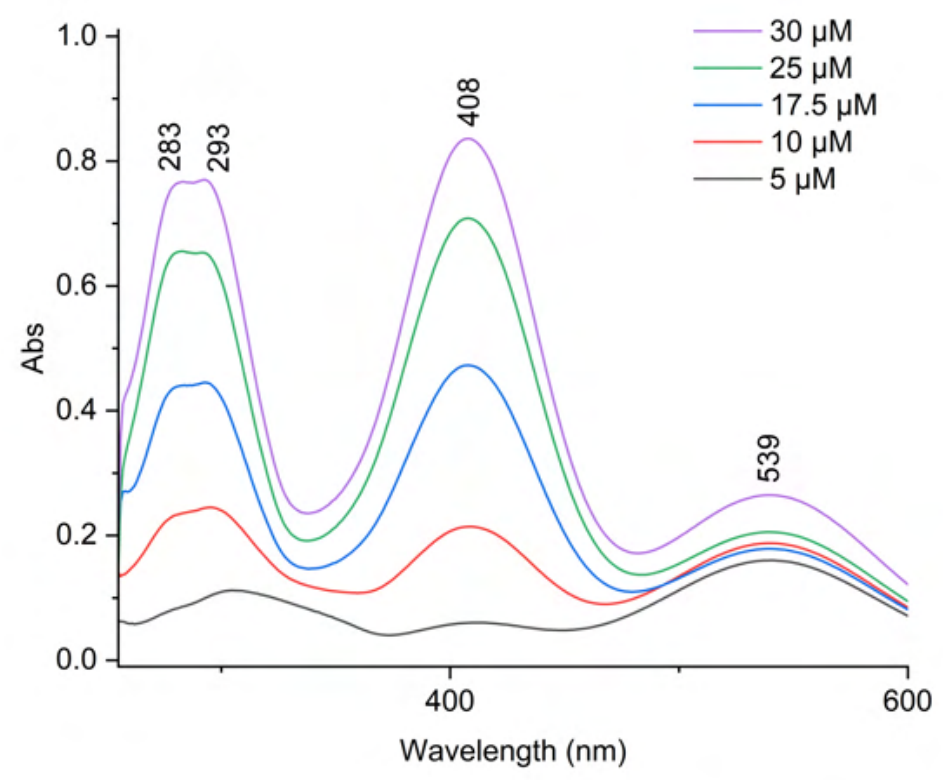

Figure S75. The UV-Vis calibration traces of 6, for concentrations ranging from 5-30 $\mu \mathrm{M}$. The wavelength of maximum absorbance is labelled $(408 \mathrm{~nm})$. These traces were collected in the absence of perchloric acid.

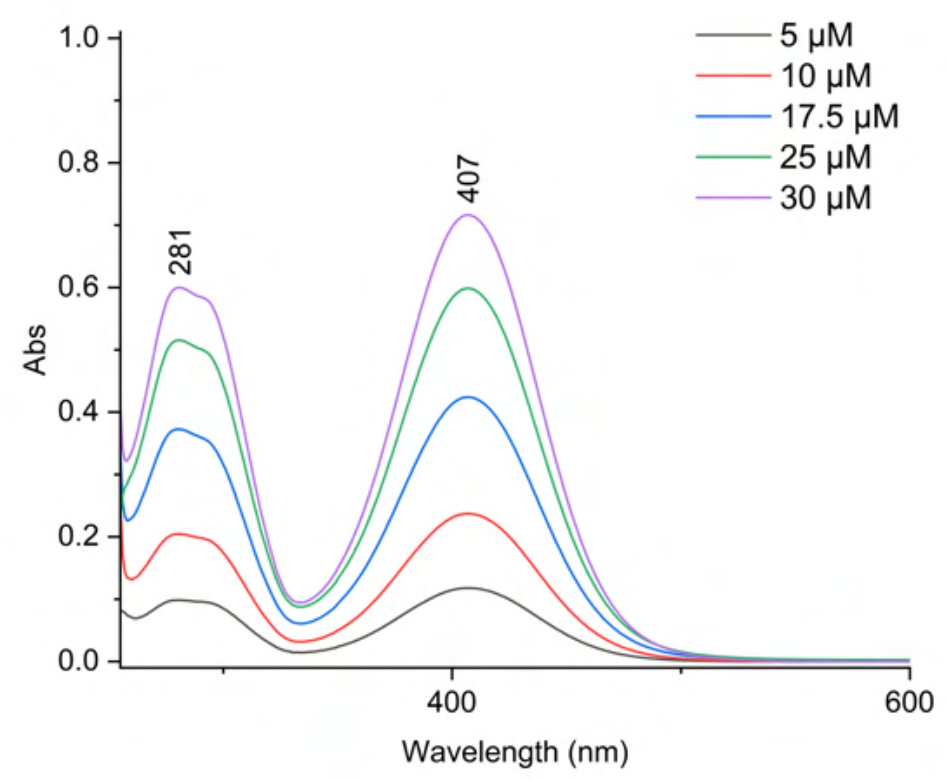

Figure S76. The UV-Vis calibration traces of 6, for concentrations ranging from 5-30 $\mu \mathrm{M}$. The wavelength of maximum absorbance is labelled $(407 \mathrm{~nm})$. These traces were collected in the presence of perchloric acid. 


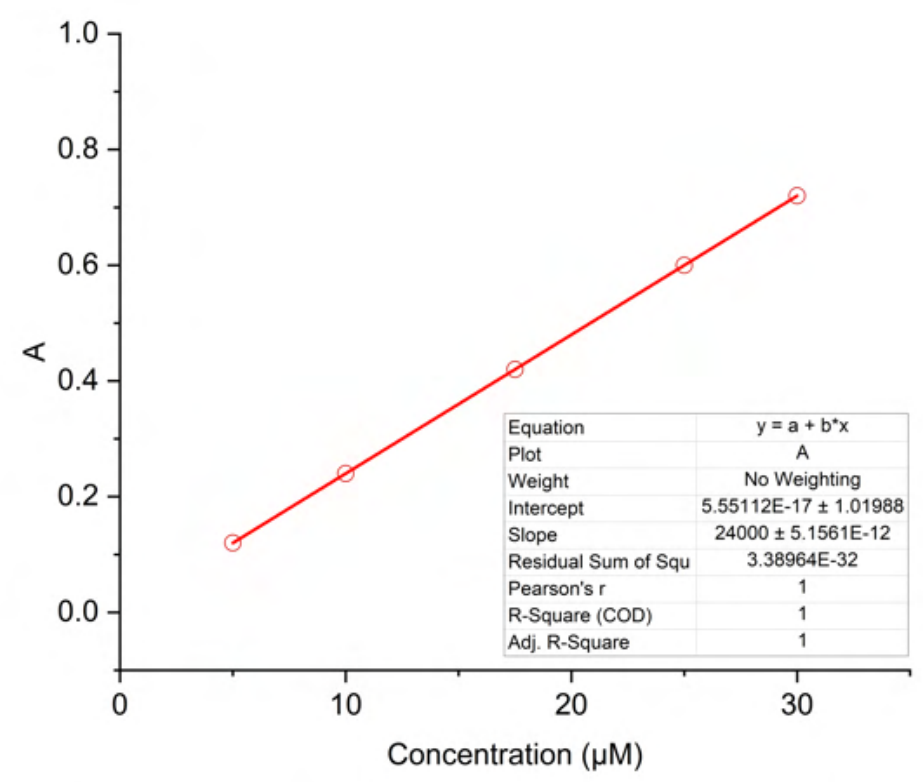

Figure S77. Plot of maximum absorbance against concentration for $\mathbf{6}$. The gradient provides a molar extinction coefficient value of $\varepsilon=24000 \mathrm{M}^{-1} \mathrm{~cm}^{-1}$.

\subsection{UV-Vis Quantification of Transporter Mass Percentage}

$\mathrm{HP}-\beta-\mathrm{CD}$ is not active in the UV-Vis region, and the UV-Vis spectrum of HP- $\beta-\mathrm{CD}$ contains no peaks or artefacts (Figure S96). Therefore, any peaks in the UV-Vis spectrum of an inclusion complex mixture were attributed solely to the transporter molecule. Spectra were collected for each of the inclusion complex mixtures (1-6, methods 1-3). A mass for each inclusion complex was recorded, and the mixtures were dissolved in $10 \mathrm{~mL}$ of DMSO. The maximum absorbance recorded from these spectra and the $\varepsilon$ calculated from the calibration fit for each transporter were input into a rearranged Beer-Lambert Law equation to calculate the concentration of transporter present in each experimental solution. This concentration value was then applied to calculate the mass of the transporter present in the experimental solution and, hence, the mass percentage of transporter present in each inclusion complex mixture compared to the mass percentage of HP- $\beta-C D$ was found.

The calculations used to quantify the mass percentage of transporter present in the inclusion complex mixture of HP- $\beta$-CD and 1 from method 3 are detailed below as an example. Initially, HP- $\beta$-CD.1 (3.77 mg, method 3) was dissolved in DMSO (10 mL), and a UV-Vis trace was collected. This spectrum's maximum absorbance was above $A=1$, so the solution was diluted 
by half, and a second spectrum was collected (Figure S80). Absorbance readings above $A=1$ are inaccurate because of the logarithmic relationship between absorption and transmittance. At $A=1$, only $10 \%$ of the available spectrometer light reaches the receiver as the sample absorbs the rest, and absorbance values rise rapidly over the remaining 10\%. A lack of light reaching the receiver means that transmittance and, thus, absorbance values become less precise. Therefore, concentrations that give absorbance measurements between a range of $A=$ 0.1-0.8 are optimal to ensure accuracy.

The maximum absorbance of the UV-Vis spectrum displayed in Figure $\mathbf{S 8 0}$ is $A_{\max }=0.777$. The gradient of the calibration fit of $\mathbf{1}$ shown in Figure $\mathbf{S 6 5}$ provided a value for the molar extinction coefficient of $\varepsilon=19588.6 \mathrm{M}^{-1} \mathrm{~cm}^{-1}$ (the gradient is equal to $\varepsilon \times l$, and $l=1 \mathrm{~cm}$ ). A 1 $\mathrm{cm}$ cuvette was also used for the UV-Vis experiments on the inclusion complex mixtures. To find the concentration of transporter, the Beer-Lambert law was rearranged to give Equation 2 as follows:

$$
c=\frac{A}{\varepsilon l}=\frac{0.77649}{\left(19588.6 M^{-1} \mathrm{~cm}^{-1}\right) \times(1 \mathrm{~cm})}=39.64 \mu \mathrm{M}
$$

Equation 2. The rearranged Beer-Lambert Law with values input for compound 1 calculations.

For the example of $\mathbf{1}$, the concentration value was doubled, accounting for the $50 \%$ dilution, to provide a transporter concentration in the original inclusion mixture sample of $79.28 \mu \mathrm{M}$ in DMSO (10 mL). The concentration value was be used to find the mass of transporter present by combining the equations below:

$$
n=\frac{m}{M}=c v
$$

Equation 3. The combined mass molarity and molar concentration equations.

Where; $n$ is the number of moles (mol), $m$ is the mass of the compound (g), $M$ is the molar mass of the compound $\left(\mathrm{gmol}^{-1}\right), c$ is the concentration $\left(\mathrm{molL}^{-1}\right)$, and $v$ is volume $(\mathrm{L})$. The molar mass of $\mathbf{1}$ is $329 \mathrm{gmol}^{-1}$. This equation was rearranged to Equation $\mathbf{4}$, and the values input, to give the mass of transporter: 


$$
m=c v M=\left(79.28 \times 10^{-6} \mathrm{~mol} \mathrm{~L}^{-1}\right) \times(0.01 \mathrm{~L}) \times\left(329 \mathrm{~g} \mathrm{~mol}^{-1}\right)=0.261 \mathrm{mg}
$$

Equation 4. The rearranged molarity equations with values input for compound $\mathbf{1}$ calculations.

The total mass of the inclusion complex mixture dissolved in DMSO was noted prior to obtaining the UV-Vis spectrum. Therefore, the mass percentage of the transporter was calculated using:

$$
\text { mass } \%=\frac{m_{\text {transporter }}}{m_{\text {total }}}=\frac{0.261 \mathrm{~g}}{3.77 \mathrm{~g}}=6.92 \%
$$

Equation 5. The mass percentage of transporter present with values input for compound $\mathbf{1}$ calculations.

The mass percentage appeared to suggest only that a small amount of transporter is present in the mixture; however, the difference in the molar mass of $\mathbf{1}$ and HP- $\beta-C D$ must be considered. The molar mass of $\mathbf{1}$ is $329 \mathrm{gmol}^{-1}$ and the average molar mass of HP- $\beta-\mathrm{CD}$ provided by the supplier, based on the estimated number of substitutions of free hydroxyl groups, is $1380 \mathrm{gmol}^{-1}$. This means a 1:1 stoichiometric mixture of the two components would provide an estimated mass percentage of $\mathbf{1}$ of $19.3 \%$. The stoichiometry of HP- $\beta$-CD-1 (method 3) was evaluated using the average molar mass of $\mathrm{HP}-\beta-\mathrm{CD}$ as follows:

$$
\frac{n_{\mathrm{HP}-\beta-\mathrm{CD}}}{n_{\mathbf{4 0 1}}}=\frac{\left(\frac{m}{M}\right)_{\mathrm{HP}-\beta-\mathrm{CD}}}{\left(\frac{m}{M}\right)_{401}}=\frac{\frac{(3.77-0.261) g}{\left(1380 \mathrm{~mol}^{-1}\right)}}{\frac{(0.261 \mathrm{~g})}{\left(329 \mathrm{~g} \mathrm{~mol}^{-1}\right)}}=\frac{\left(2.54 \times 10^{-3} \mathrm{~mol}\right)}{\left(7.9 \times 10^{-4} \mathrm{~mol}\right)}=3.21
$$

Equation 6. The stoichiometric ratio of $\mathrm{HP}-\beta-\mathrm{CD}$ :transporter present in the inclusion complex mixtures with values input for compound 1 calculations.

Therefore, there is approximately a 3:1 molar ratio of $\mathrm{HP}-\beta-\mathrm{CD}$ to $\mathbf{1}$ present in the inclusion complex mixture of HP- $\beta$-CD-1 (method 3). Calculations for the other compounds and inclusion methods are detailed below, and the results for each method are displayed for comparison in Table S10. In general, method 3 provided the highest level of transporter inclusion. Microwave irradiation has appeared as a reliable and effective method for the 
formulation of inclusion complexes. ${ }^{17,} 18$ Method 1 (kneading) resulted in the lowest amount of guest inclusion, which is in agreement with other comparisons of inclusion methods reported in the literature. ${ }^{19,20}$

Table S10. The mass percentage of transporter present in the inclusion complex mixtures formed between HP- $\beta-\mathrm{CD}$ and compounds 1-6 via methods 1-3.

\begin{tabular}{c|ccc} 
Compound & Inclusion Method 1 & Inclusion Method 2 & Inclusion Method 3 \\
\hline $\mathbf{1}$ & $3.05 \%$ & $5.34 \%$ & $6.92 \%$ \\
$\mathbf{2}$ & $4.17 \%$ & $3.96 \%$ & $6.46 \%$ \\
$\mathbf{3}$ & $-\mathrm{a}$ & $-\mathrm{a}$ & $-\mathrm{a}$ \\
$\mathbf{4}$ & $2.94 \%$ & $4.83 \%$ & $6.05 \%$ \\
$\mathbf{5}$ & $1.82 \%$ & $4.72 \%$ & $0.92 \%$ \\
$\mathbf{6}$ & $5.51 \%{ }^{\mathrm{b}}$ & $6.84 \% \mathrm{o}^{\mathrm{b}}$ & $1.83 \%{ }^{\mathrm{b}}$
\end{tabular}

a Maximum absorbance peak in the UV-Vis spectrum is not large enough to quantify the mass percentage. ${ }^{\mathrm{b}}$

Calculated in the presence of $0.01 \mathrm{M}$ of perchloric acid.

Inclusion complex mixtures containing more than $5 \%$ of 5 were not formed successfully, which seems likely to result from steric effects. This molecule lacks a methyl spacer to separate the hydrogen bond donor from the adamantyl group. The proximity of the adamantane to the bulky thiourea sulfur atom may sterically inhibit the approach of $\mathrm{HP}-\beta-\mathrm{CD}$ and the inclusion of this part of the molecule inside the macrocycle. Inclusion complexes with compound 3 did not form whatsoever, and the process seems to be impacted by the limited solubility of the compound in ethanol. It appears that the inclusion process is more effective when both components are in the solution phase, and the limited solubility of compound 3 had already been encountered during $\mathrm{Cl}^{-}$transport experiments. Compound 6 lacks a methyl spacer; however, it possesses a higher intrinsic solubility than 3 in organic solvents. This was evidenced during the synthesis of these compounds, where 6 did not crash out of the methanolic solvent, and was also reflected in the inclusion attempts, where a complex was successfully formed between 6 and HP- $\beta-C D$. 


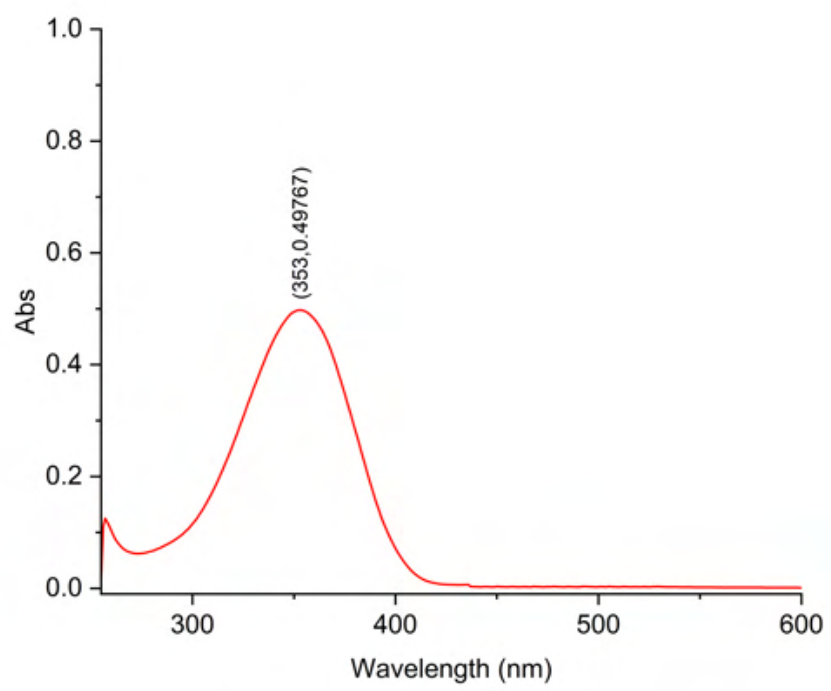

Figure S78. The UV-Vis spectrum of HP- $\beta-C D \cdot 1$ (method 1, $4.11 \mathrm{mg})$ in DMSO (10 mL).

$A_{\max }=0.49767, \varepsilon=19588.6 \mathrm{M}^{-1} \mathrm{~cm}^{-1}$

$c=25.41 \mu \mathrm{M}$

Solution was diluted by 1.5 so true concentration $=38.12 \mu \mathrm{M}$

$m=\left(38.12 \times 10^{-6}\right) \times 0.01 \times 329=0.125 \mathrm{mg}$

$\operatorname{mass} \%=3.05 \%$ 


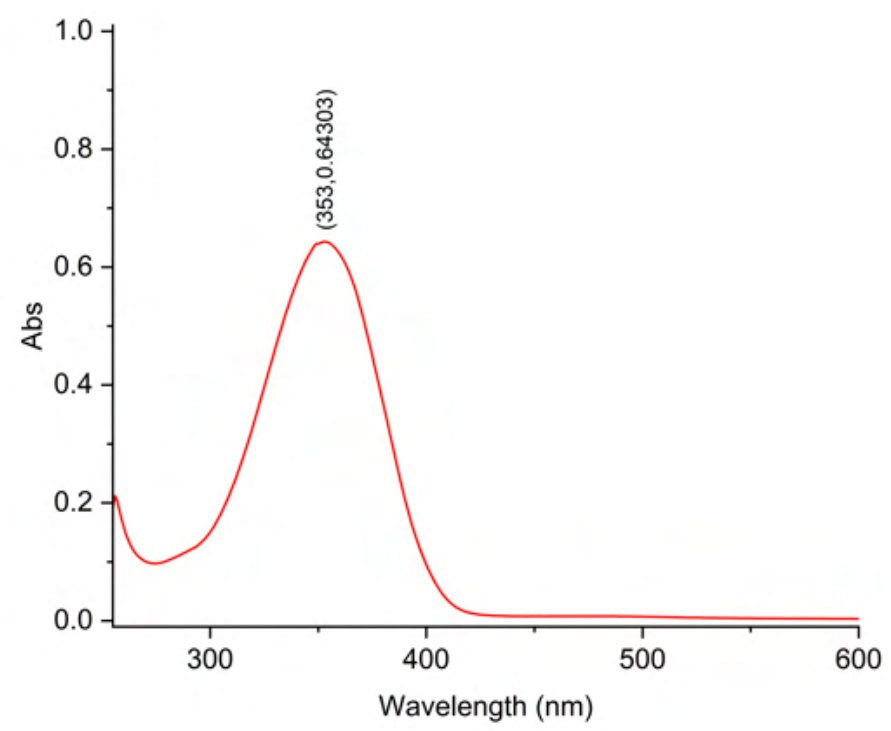

Figure S79. The UV-Vis spectrum of HP- $\beta-C D \cdot 1$ (method 2, $4.04 \mathrm{mg}$ ) in DMSO (10 mL).

$A_{\max }=0.64303, \varepsilon=19588.6 \mathrm{M}^{-1} \mathrm{~cm}^{-1}$

$c=32.83 \mu \mathrm{M}$

Solution was diluted by 2 so true concentration $=65.65 \mu \mathrm{M}$

$m=\left(65.65 \times 10^{-6}\right) \times 0.01 \times 329=0.216 \mathrm{mg}$

$\operatorname{mass} \%=5.34 \%$ 


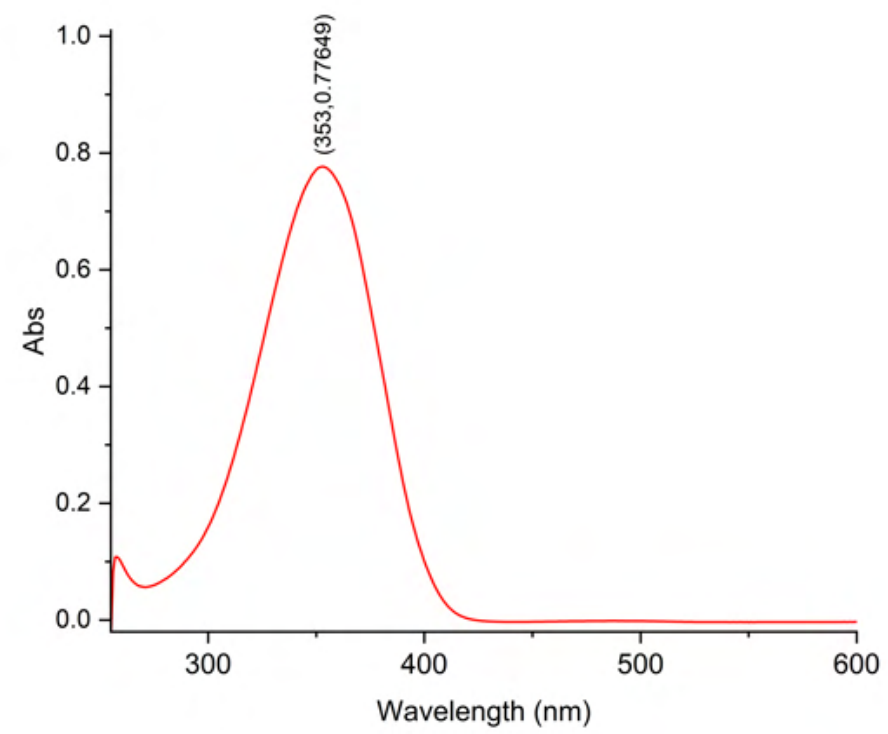

Figure S80. The UV-Vis spectrum of HP- $\beta-C D \cdot 1$ (method 3, $3.77 \mathrm{mg})$ in DMSO $(10 \mathrm{~mL})$.

$A_{\max }=0.77649, \varepsilon=19588.6 \mathrm{M}^{-1} \mathrm{~cm}^{-1}$

$c=39.64 \mu \mathrm{M}$

Solution was diluted by 2 so true concentration $=79.28 \mu \mathrm{M}$

$m=\left(79.28 \times 10^{-6}\right) \times 0.01 \times 329=0.261 \mathrm{mg}$

$\operatorname{mass} \%=6.92 \%$ 


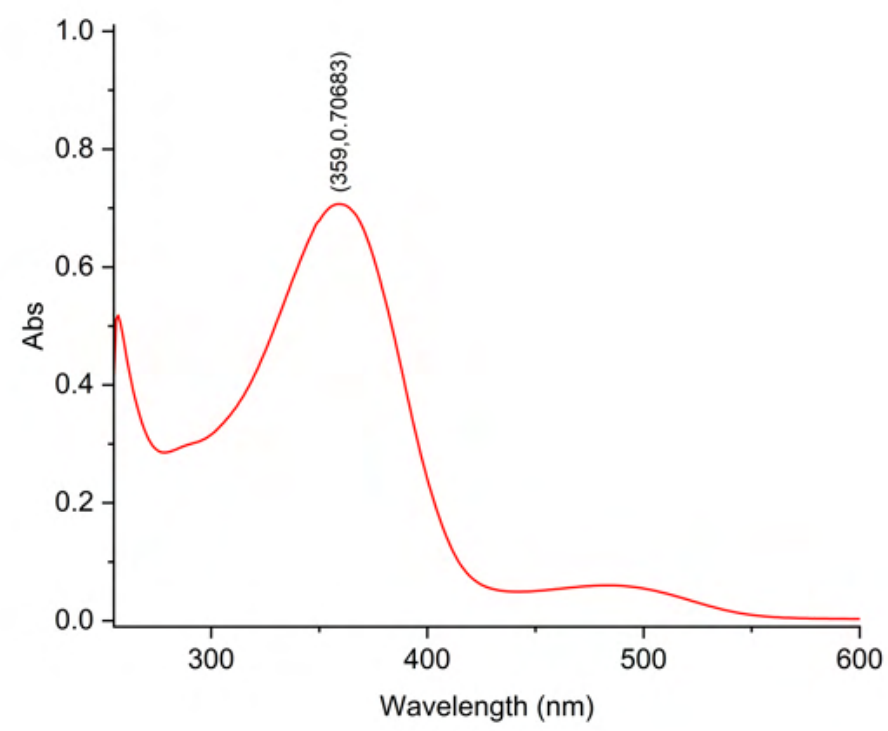

Figure S81. The UV-Vis spectrum of HP- $\beta-C D \cdot 2($ method $1,1.77 \mathrm{mg})$ in DMSO $(6 \mathrm{~mL})$.

$$
\begin{aligned}
& A_{\max }=0.70683, \varepsilon=19586.5 \mathrm{M}^{-1} \mathrm{~cm}^{-1} \\
& c=36.09 \mu \mathrm{M} \\
& m=\left(36.09 \times 10^{-6}\right) \times 0.006 \times 341=0.0738 \mathrm{mg} \\
& \text { mass } \%=4.17 \%
\end{aligned}
$$




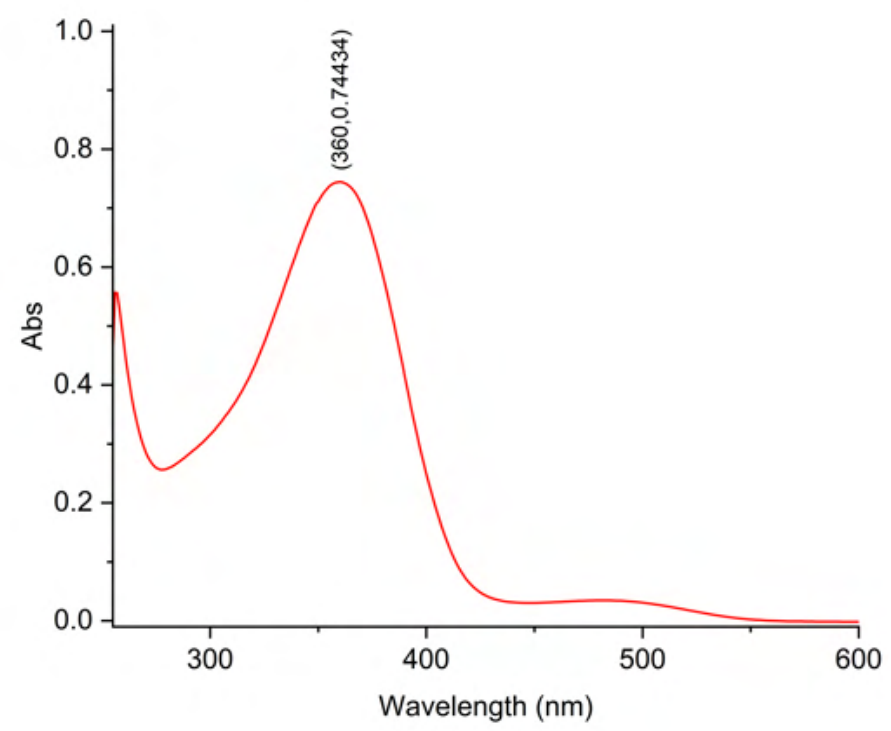

Figure S82. The UV-Vis spectrum of HP- $\beta-C D \cdot 2$ (method 2, 3.27 mg) in DMSO (10 mL).

$$
\begin{aligned}
& A_{\max }=0.74434, \varepsilon=19586.5 \mathrm{M}^{-1} \mathrm{~cm}^{-1} \\
& c=38.00 \mu \mathrm{M} \\
& m=\left(38.00 \times 10^{-6}\right) \times 0.01 \times 341=0.129 \mathrm{mg} \\
& \text { mass } \%=3.96 \%
\end{aligned}
$$




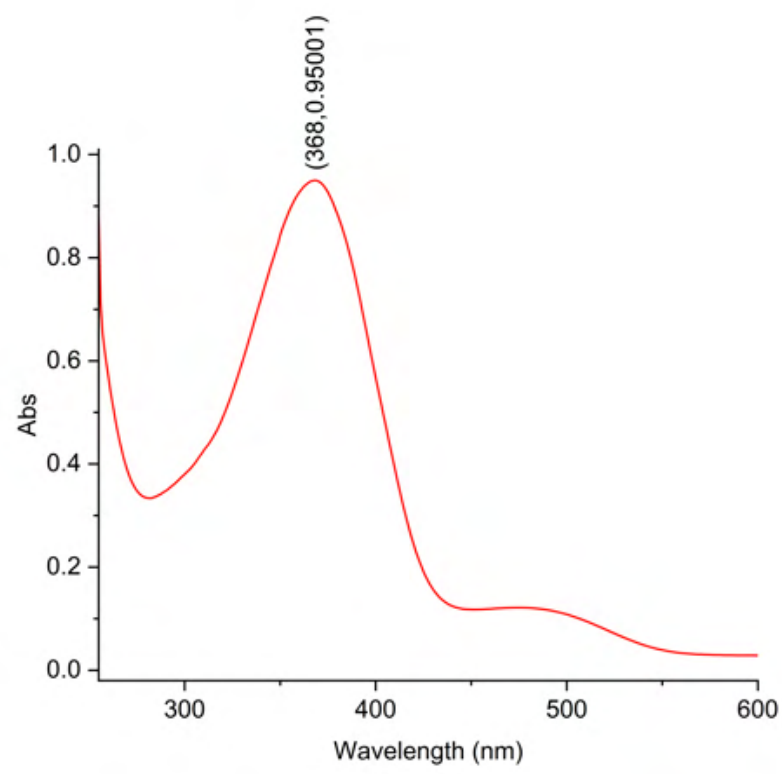

Figure S83.The UV-Vis spectrum of HP- $\beta$-CD 2 (method 3, $1.28 \mathrm{mg}$ ) in DMSO $(5 \mathrm{~mL})$.

$A_{\max }=0.95001, \varepsilon=19586.5 \mathrm{M}^{-1} \mathrm{~cm}^{-1}$

$c=48.50 \mu \mathrm{M}$

$m=\left(48.50 \times 10^{-6}\right) \times 0.005 \times 341=0.0827 \mathrm{mg}$

$\operatorname{mass} \%=6.46 \%$ 


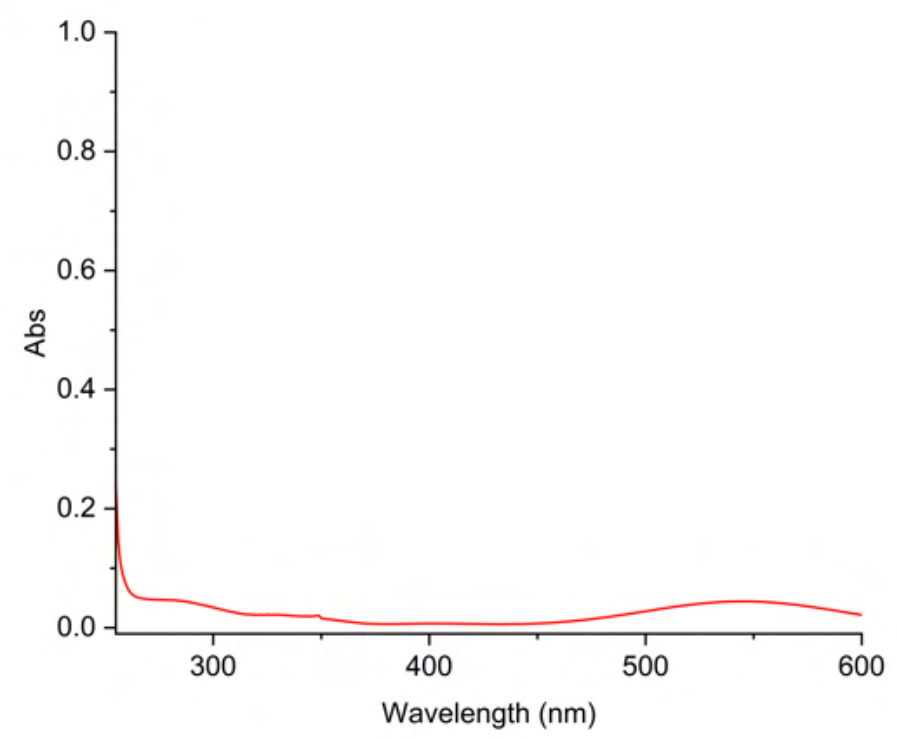

Figure S84. The UV-Vis spectrum of HP- $\beta-C D \cdot 3$ (method 1, $4.00 \mathrm{mg}$ ) in DMSO (10 mL). The $A_{\max }$ for this compound was too low for calculations to be performed.

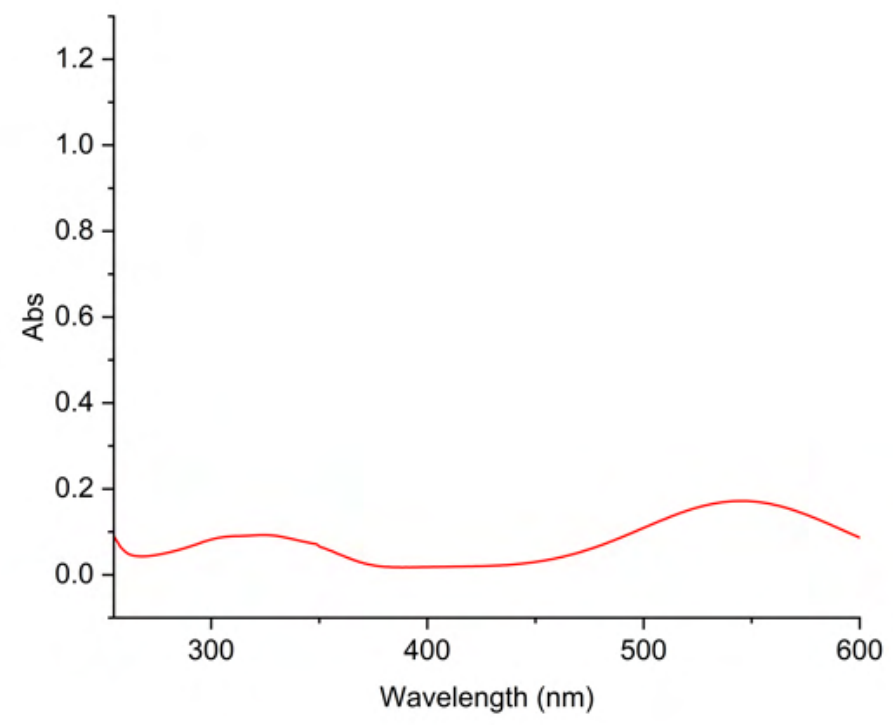

Figure S85. The UV-Vis spectrum of $\mathrm{HP}-\beta-\mathrm{CD} \cdot 3$ (method $2,5.17 \mathrm{mg}$ ) in DMSO $(10 \mathrm{~mL})$. The $A_{\max }$ for this compound was too low for calculations to be performed. 


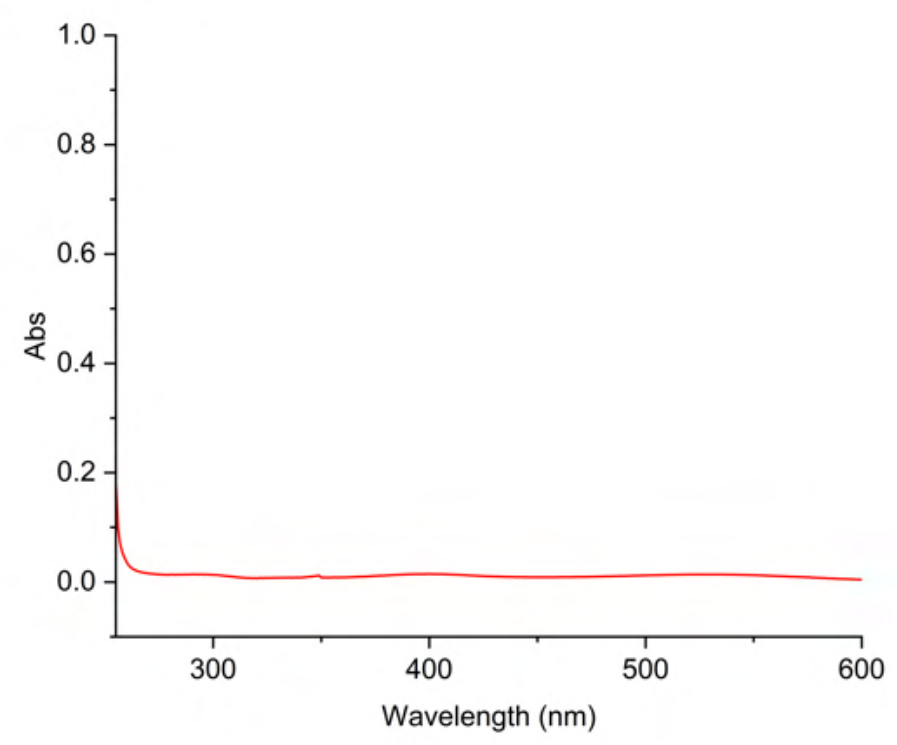

Figure S86. The UV-Vis spectrum of HP- $\beta-C D \cdot 3$ (method 3, $4.31 \mathrm{mg}$ ) in DMSO (10 mL). The $A_{\max }$ for this compound was too low for calculations to be performed. 


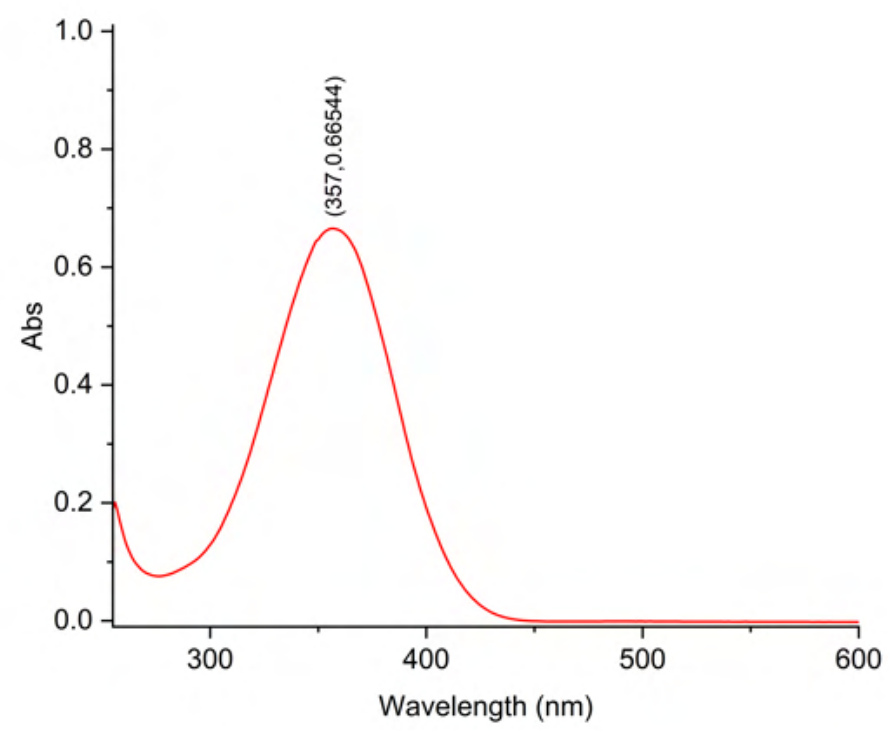

Figure S87. The UV-Vis spectrum of HP- $\beta-C D \cdot 4$ (method 1, $4.42 \mathrm{mg})$ in DMSO (10 mL).

$A_{\max }=0.66544, \varepsilon=24165.0 \mathrm{M}^{-1} \mathrm{~cm}^{-1}$

$c=27.54 \mu \mathrm{M}$

Solution was diluted by 1.5 so true concentration $=41.31 \mu \mathrm{M}$

$m=\left(41.31 \times 10^{-6}\right) \times 0.01 \times 315=0.130 \mathrm{mg}$

$\operatorname{mass} \%=2.94 \%$ 


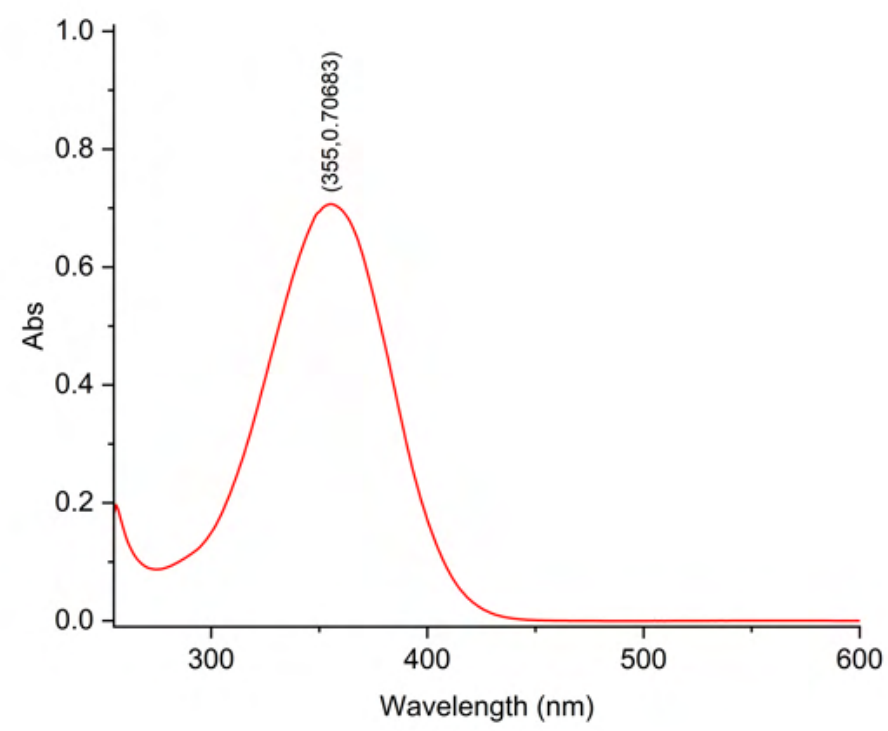

Figure S88. The UV-Vis spectrum of HP- $\beta-C D \cdot 4$ (method 2, $3.82 \mathrm{mg})$ in DMSO (10 mL).

$A_{\max }=0.70683, \varepsilon=24165.0 \mathrm{M}^{-1} \mathrm{~cm}^{-1}$

$c=29.25 \mu \mathrm{M}$

Solution was diluted by 2 so true concentration $=58.50 \mu \mathrm{M}$

$m=\left(58.5 \times 10^{-6}\right) \times 0.01 \times 315=0.184 \mathrm{mg}$

$\operatorname{mass} \%=4.82 \%$ 


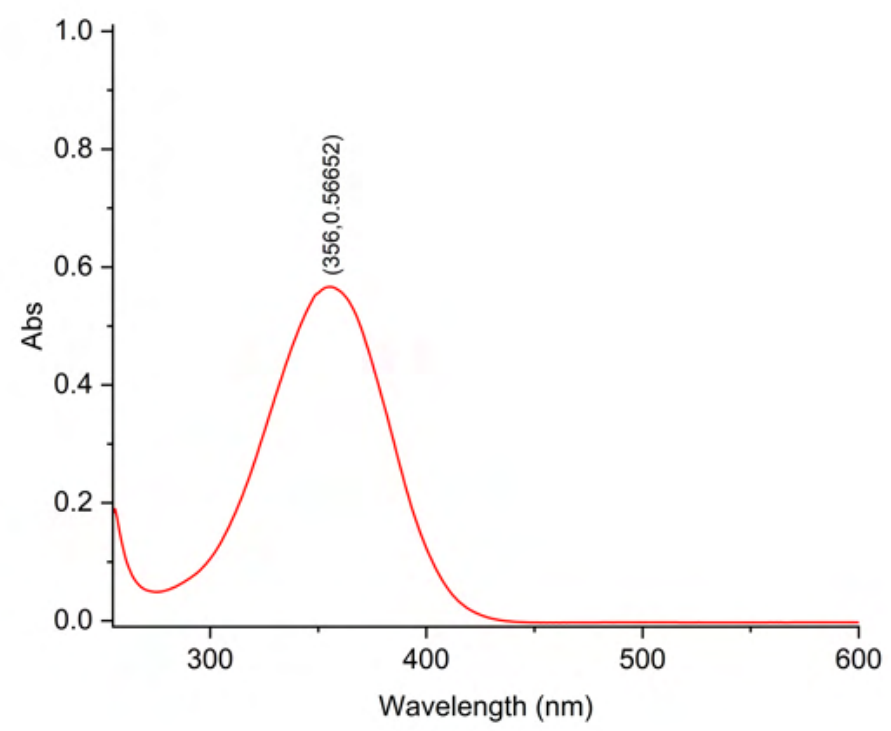

Figure S89. The UV-Vis spectrum of HP- $\beta-C D \cdot 4$ (method 3, $3.66 \mathrm{mg})$ in DMSO (10 mL).

$A_{\max }=0.56652, \varepsilon=24165.0 \mathrm{M}^{-1} \mathrm{~cm}^{-1}$

$c=23.44 \mu \mathrm{M}$

Solution was diluted by 3 so true concentration $=70.33 \mu \mathrm{M}$

$m=\left(70.33 \times 10^{-6}\right) \times 0.01 \times 315=0.222 \mathrm{mg}$

$\operatorname{mass} \%=6.05 \%$ 


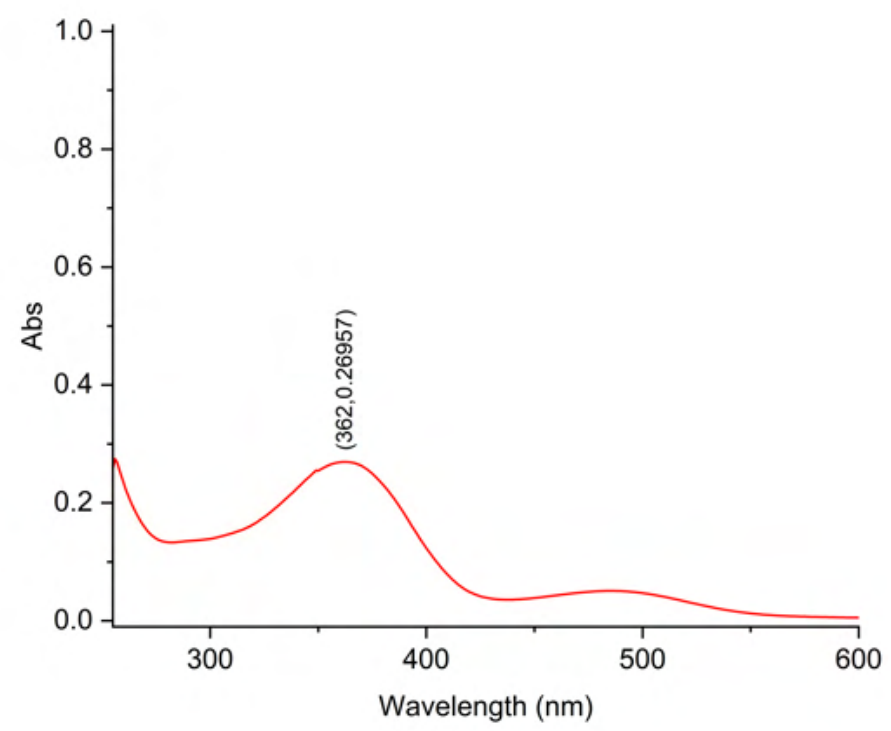

Figure S90. The UV-Vis spectrum of HP- $\beta-C D \cdot 5$ (method 1, $3.13 \mathrm{mg})$ in DMSO (10 mL).

$$
\begin{aligned}
& A_{\max }=0.26957, \varepsilon=15683.3 \mathrm{M}^{-1} \mathrm{~cm}^{-1} \\
& \mathcal{c}=17.19 \mu \mathrm{M} \\
& m=\left(17.19 \times 10^{-6}\right) \times 0.01 \times 331=0.0569 \mathrm{mg} \\
& \text { mass } \%=1.82 \%
\end{aligned}
$$




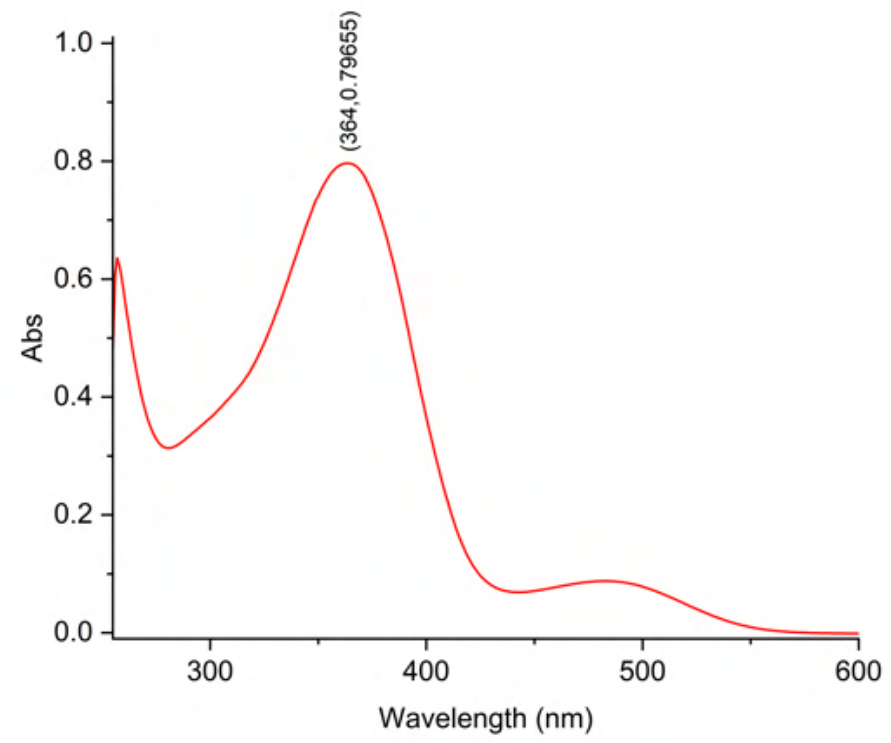

Figure S91. The UV-Vis spectrum of HP- $\beta-C D \cdot 5$ (method 2, $3.56 \mathrm{mg})$ in DMSO (10 mL).

$$
\begin{aligned}
& A_{\max }=0.79655, \varepsilon=15683.3 \mathrm{M}^{-1} \mathrm{~cm}^{-1} \\
& c=50.79 \mu \mathrm{M} \\
& m=\left(50.79 \times 10^{-6}\right) \times 0.01 \times 331=0.168 \mathrm{mg}
\end{aligned}
$$

mass $\%=4.72 \%$ 


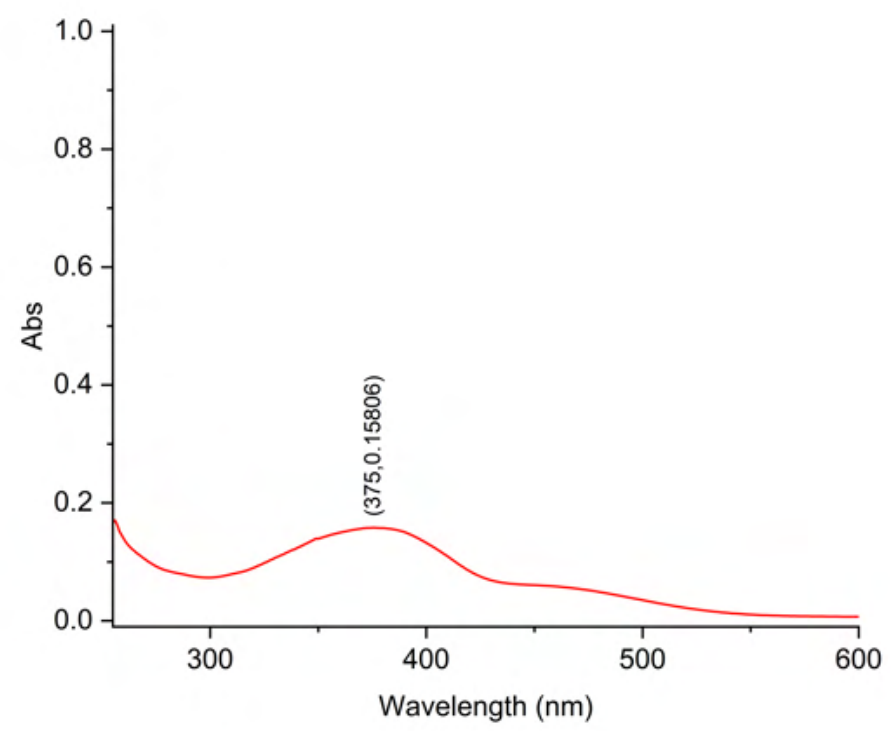

Figure S92. The UV-Vis spectrum of HP- $\beta-C D \cdot 5$ (method 3, $3.62 \mathrm{mg})$ in DMSO (10 mL).

$$
\begin{aligned}
& A_{\max }=0.15806, \varepsilon=15683.3 \mathrm{M}^{-1} \mathrm{~cm}^{-1} \\
& c=10.08 \mu \mathrm{M} \\
& m=\left(50.79 \times 10^{-6}\right) \times 0.01 \times 331=0.0334 \mathrm{mg}
\end{aligned}
$$$$
\text { mass } \%=0.92 \%
$$ 


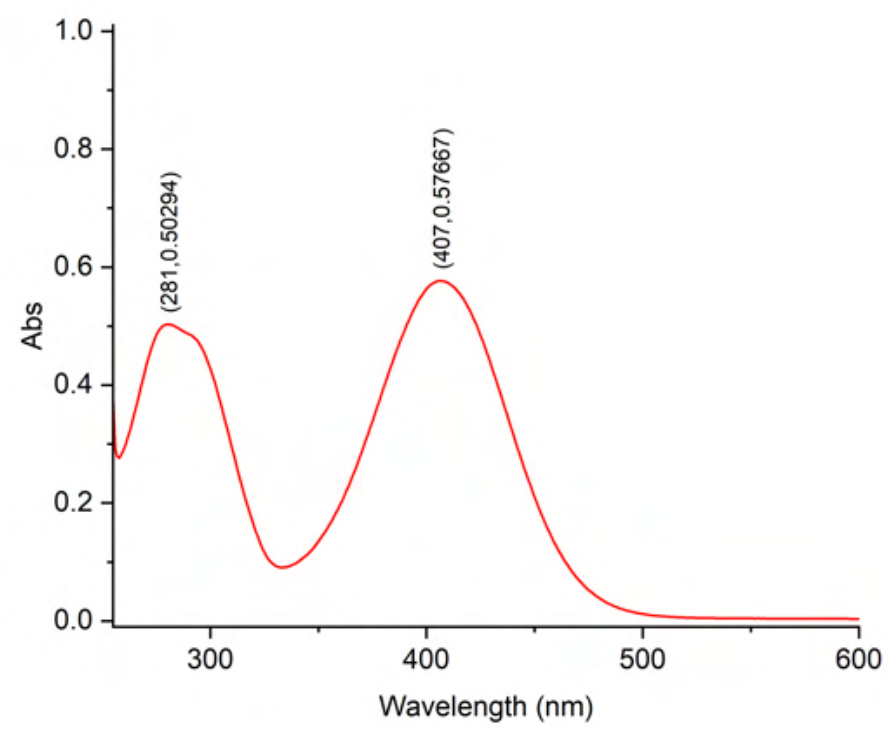

Figure S93. The UV-Vis spectrum of HP- $\beta-C D \cdot 6$ (method $1,1.60 \mathrm{mg}$ ) in DMSO (10 mL), in the presence of perchloric acid $(0.01 \mathrm{M})$.

$A_{\max }=0.57667, \varepsilon=24000.0 \mathrm{M}^{-1} \mathrm{~cm}^{-1}$

$c=24.03 \mu \mathrm{M}$

$m=\left(24.03 \times 10^{-6}\right) \times 0.01 \times 367=0.0887 \mathrm{mg}$

$\operatorname{mass} \%=5.51 \%$ 


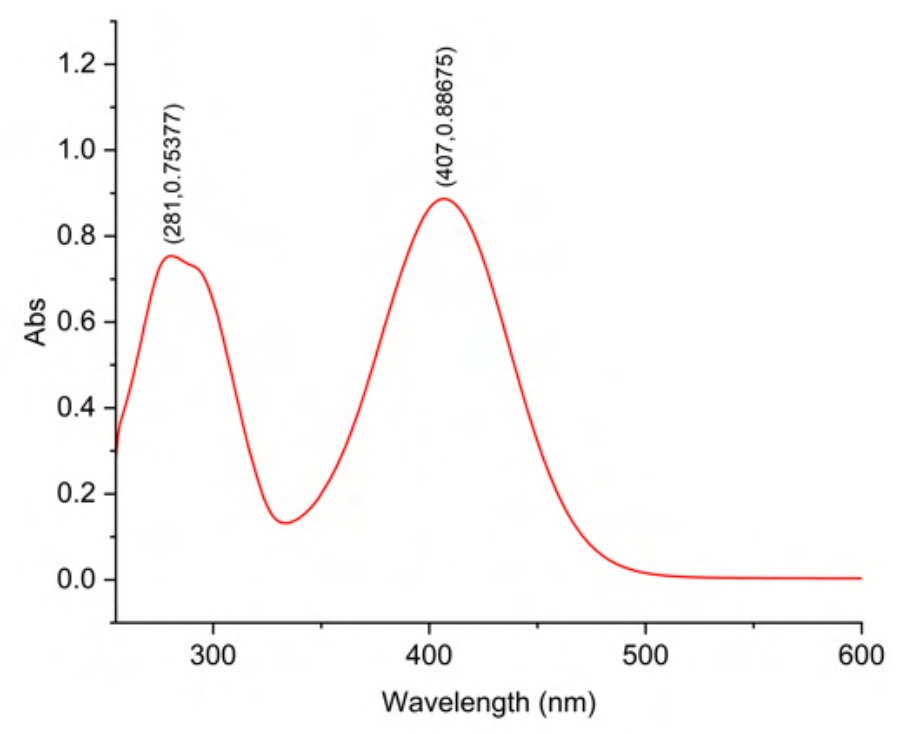

Figure S94. The UV-Vis spectrum of HP- $\beta-C D \cdot 6$ (method 2, $1.99 \mathrm{mg})$ in DMSO $(10 \mathrm{~mL})$, in the presence of perchloric acid (0.01 M).

$A_{\max }=0.88675, \varepsilon=24000.0 \mathrm{M}^{-1} \mathrm{~cm}^{-1}$

$c=36.95 \mu \mathrm{M}$

$m=\left(36.95 \times 10^{-6}\right) \times 0.01 \times 367=0.136 \mathrm{mg}$

$\operatorname{mass} \%=6.81 \%$ 


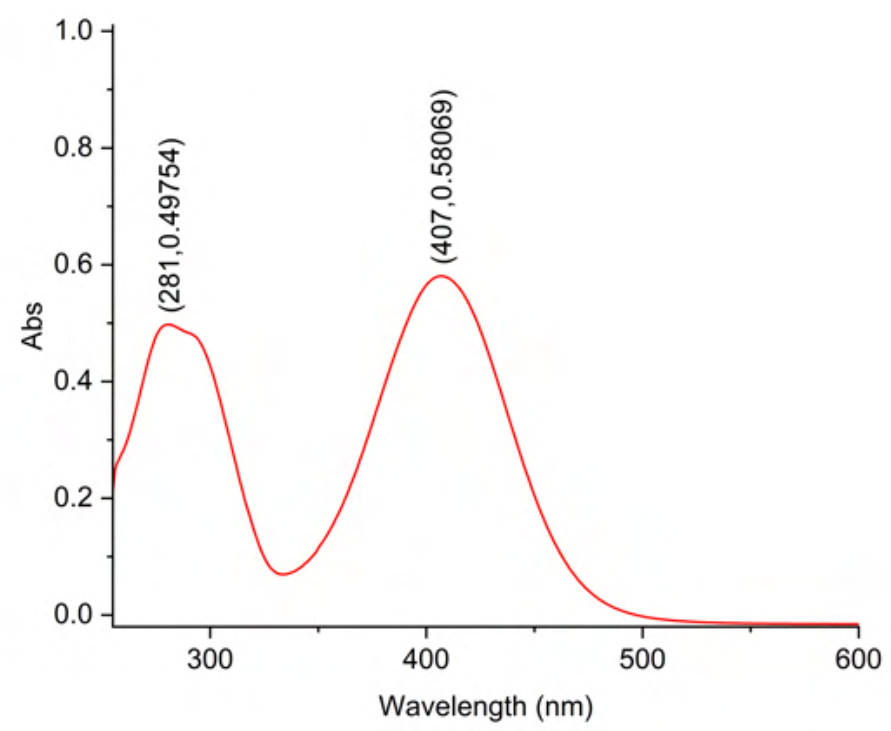

Figure S95. The UV-Vis spectrum of HP- $\beta-C D \cdot 6$ (method 3, $4.85 \mathrm{mg}$ ) in DMSO (10 mL), in the presence of perchloric acid $(0.01 \mathrm{M})$.

$A_{\max }=0.58069, \varepsilon=24000.0 \mathrm{M}^{-1} \mathrm{~cm}^{-1}$

$c=24.42 \mu \mathrm{M}$

$m=\left(24.42 \times 10^{-6}\right) \times 0.01 \times 367=0.088 \mathrm{mg}$

$\operatorname{mass} \%=1.83 \%$ 


\subsection{UV-Vis of Inclusion Complexes in $\mathrm{H}_{2} \mathrm{O}$}

$\mathrm{UV}$-Vis spectra of the four inclusion complex mixtures were collected in $\mathrm{H}_{2} \mathrm{O}$. For the mass percentage quantifications, it did not matter whether the inclusion complex was retained when dissolved in DMSO as only the concentration of compound present was necessary for calculations. A UV-Vis spectrum of free transporter 1 in $\mathrm{H}_{2} \mathrm{O}$ could not be obtained due to the poor solubility of the compound in this solvent. Therefore, UV-Vis absorbance can be attributed to the transporter molecule when the complex is dissolved in water would indicate an inclusion complex has been formed.

The similarity in the absorbance profile of the inclusion mixture spectra in $\mathrm{H}_{2} \mathrm{O}$ and the corresponding free transporter spectrum in DMSO heavily suggested inclusion complexes had been fabricated successfully. The small peak at higher wavelengths in the DMSO spectrum is due to a slight deprotonation. ofiob] The UV-Vis spectrum of HP- $\beta-C D \cdot 6$ obtained in $\mathrm{H}_{2} \mathrm{O}$ contains a large amount of noise, attributed to the formation of microdispersions in the experimental solution. This could potentially be due to the partial decomplexation and subsequent precipitation of the transporter. The overlaid spectra can be viewed below.

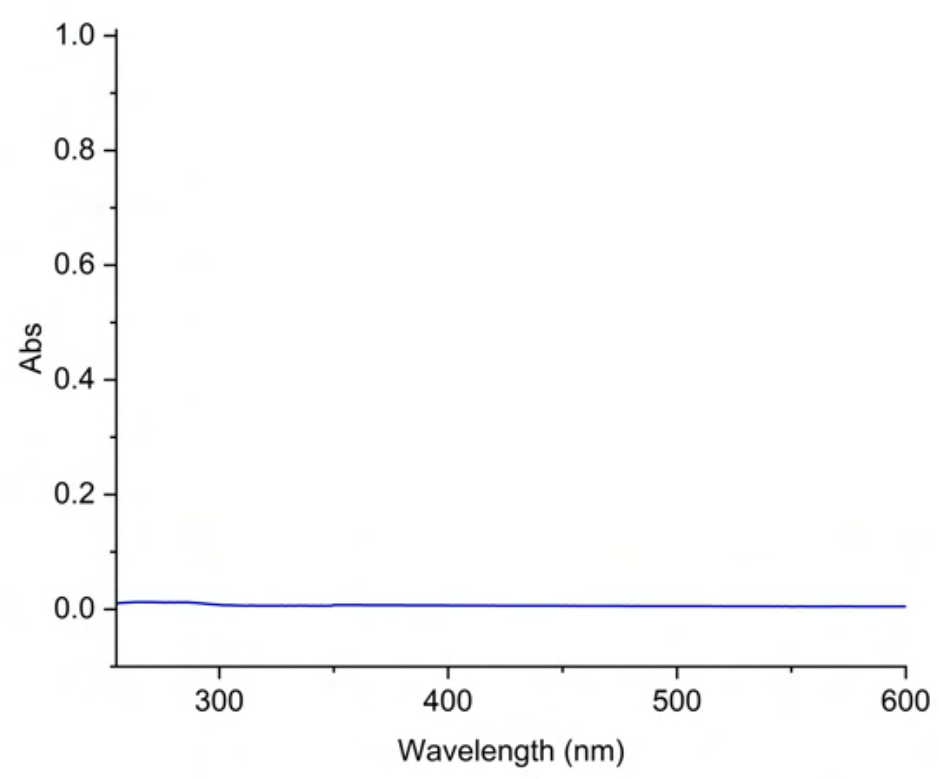

Figure S96. The UV-Vis trace of HP- $\beta-C D$ in water. 


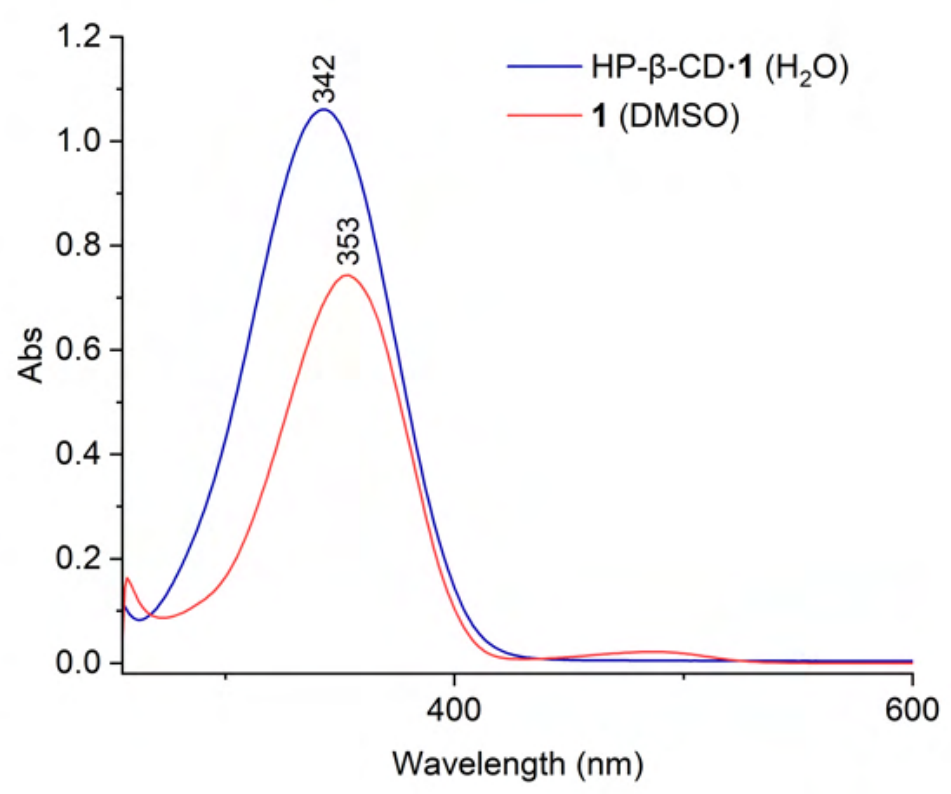

Figure S97. An overlay of the UV-Vis traces of free transporter 1 dissolved in DMSO (red) and of inclusion complex HP- $\beta-C D \cdot 1$ in water (blue).

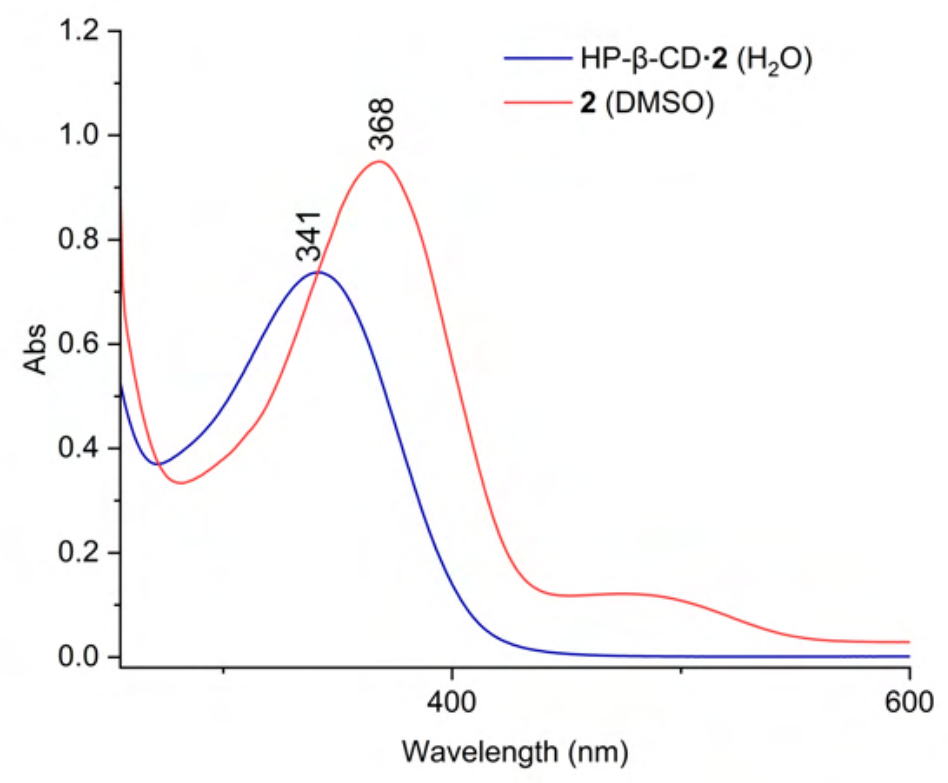

Figure S98. An overlay of the UV-Vis traces of free transporter 2 dissolved in DMSO (red) and of inclusion complex HP- $\beta-C D \cdot 2$ in water (blue). 


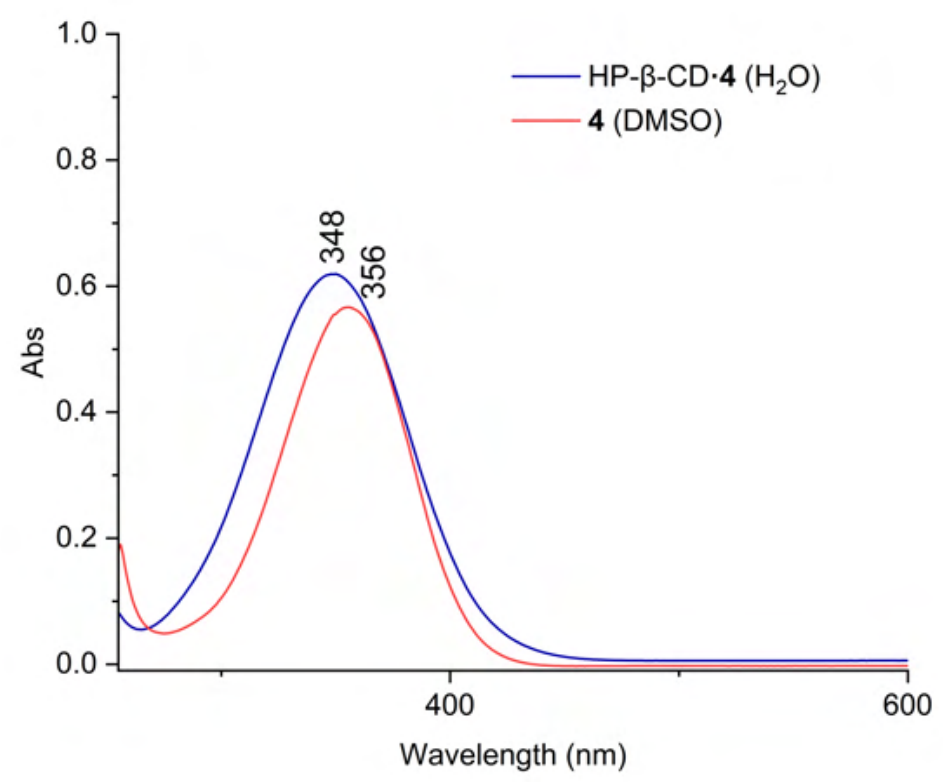

Figure S99. An overlay of the UV-Vis traces of free transporter 4 dissolved in DMSO (red) and of inclusion complex HP- $\beta-C D \cdot 4$ in water (blue).

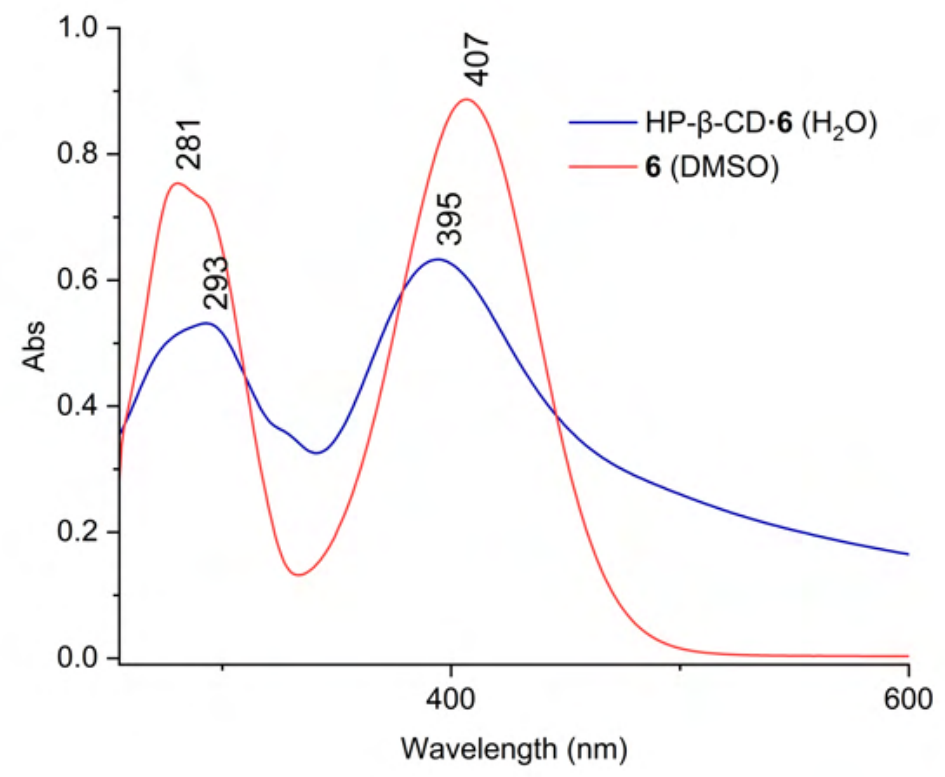

Figure S100. An overlay of the UV-Vis traces of free transporter 6 dissolved in DMSO (red) and of inclusion complex HP- $\beta-C D \cdot 6$ in water (blue). The large amount of noise in the spectrum of HP- $\beta$-CD.6 was attributed to the formation of microdispersions in the experimental solution. 


\section{Infrared Spectroscopy Analysis}

Infrared spectroscopy was also employed to examine the inclusion complexes. This technique can be used to identify perturbations in the vibrational modes of the CD host and the guest molecule, which may be due to interactions between the two molecules in the solid-state. ${ }^{22} \mathrm{~A}$ decrease in intensity, a shift in wavenumber, or the disappearance of characteristic stretches associated with the guest can all provide evidence towards the formation of an inclusion complex due to restriction of the guests stretching vibrations when included within the HP$\beta$-CD cavity. Attenuated total reflectance (ATR) Fourier-transform infrared (FTIR) spectra were recorded for free $\mathrm{HP}-\beta-\mathrm{CD}$, free transporters 1, 2, 4, and 6, and each inclusion complex with the respective transporter. Additionally, ATR spectrums were collected for a physical mixture (P.M) of each transporter and $\mathrm{HP}-\beta-\mathrm{CD}$, prepared by combining an equimolar amount of transporter and HP- $\beta-C D$ in a mortar and pestle before grinding for $10 \mathrm{~min}$, yielding an amorphous and homogenous solid mixture.

The FTIR spectra relevant to compound 1 can be viewed in Figure S105. The FTIR spectrum of HP- $\beta$-CD (Figure S101) showed prominent absorption bands at $3311 \mathrm{~cm}^{-1}(\mathrm{O}-\mathrm{H}$ stretching vibrations), 2973, and $2915 \mathrm{~cm}^{-1}$ (C-H stretching vibrations), and 1151, 1077 and $1023 \mathrm{~cm}^{-1}$ (C-H, C-O stretching vibration). The spectrum of 1 (Figure S102) consisted of a sharp absorption band at $3357 \mathrm{~cm}^{-1}$ (urea N-H stretching vibration), two strong peaks at 2897 and $2847 \mathrm{~cm}^{-1}$ (adamantyl and methyl spacer $\mathrm{C}-\mathrm{H}$ stretching vibrations), an absorption band at 
$1671 \mathrm{~cm}^{-1}$ (urea $\mathrm{C}=\mathrm{O}$ stretching vibration) and prominent bands at 1547 and $1329 \mathrm{~cm}^{-1}$ which denote the asymmetric and symmetric stretches of the $\mathrm{NO}_{2}$ group. Peaks at 1223, 846, and 638 $\mathrm{cm}^{-1}$ are associated with aromatic ring vibrations.

The P.M spectrum (Figure S103) contained stretches associated with both compounds and, in general, the bands appeared at the same frequencies as those in the spectra of the single components. At higher wavenumbers (above $2800 \mathrm{~cm}^{-1}$ ), the bands associated with 1 appeared more prominently. The FTIR spectrum of HP- $\beta-\mathrm{CD} \cdot \mathbf{1}$ (Figure S104) closely resembles the spectrum of HP- $\beta$-CD as a free host molecule. There is a high mass content of HP- $\beta$-CD present in the inclusion complex, and bands associated with this component are much more intense, leading to overlapping and obscuring of the bands associated with 1..22 Previous studies have reported that stretches found between $3600-2800 \mathrm{~cm}^{-1}$ rarely provide evidence for the formation of an inclusion complex due to the intensity of the HP- $\beta$-CD bands in this region. ${ }^{23,24}$ This is unfortunate, as the bands in the spectra of free $\mathbf{1}$ (Figure S102) attributed to the adamantyl $\mathrm{C}-\mathrm{H}$ stretch vibrations, which should be affected by the inclusion process, were reported within this region.

The peak at $1325 \mathrm{~cm}^{-1}$ in the spectrum of HP- $\beta-C D \cdot 1$ can be attributed to the symmetric stretch of the $\mathrm{NO}_{2}$ group present in $\mathbf{1}$ and confirms the presence of the transporter in the inclusion complex. The intensity of the band at $1231 \mathrm{~cm}^{-1}$ (aromatic stretch) is greater in the spectrum for the free guest molecule compared to the band at $1325 \mathrm{~cm}^{-1}\left(\mathrm{NO}_{2}\right.$ group stretch), which is diminished in the spectrum of HP- $\beta-C D \cdot 1$. The peak at $1231 \mathrm{~cm}^{-1}$ can be attributed to the stretch vibration of the aromatic $\mathrm{C}-\mathrm{H}$ bonds. A decrease in the intensity of this band suggests that vibration of the aromatic $\mathrm{C}-\mathrm{H}$ bonds is restricted by interactions with the HP- $\beta$-CD cavity, whereas the $\mathrm{NO}_{2}$ bonds remain unaffected. The complete disappearance of the band at 1671 $\mathrm{cm}^{-1}$ indicates that vibration of the urea carbonyl may also be inhibited due to interactions with the HP- $\beta-C D$ cavity. However, this could also be an artefact of the small mass amount of transporter present in the complex.

The FTIR spectra relevant to compound 2 are displayed in Figure S109. The spectrum of free 2 (Figure S106) consisted of a sharp band at $3362 \mathrm{~cm}^{-1}$ attributed to the thiourea N-H stretch vibration, peaks at 2987 and $2893 \mathrm{~cm}^{-1}$ corresponding to the adamantyl and methyl spacer $\mathrm{C}-\mathrm{H}$ stretch vibrations. The same spectra also exhibited $\mathrm{NO}_{2}$ group vibrations at 1509 and 
$1337 \mathrm{~cm}^{-1}$, a characteristic $\mathrm{C}=\mathrm{S}$ vibration band at $1256 \mathrm{~cm}^{-1}$, and several other aromatic vibration peaks. Interestingly, the $\mathrm{N}-\mathrm{H}$ vibration band was not visible in the spectrum of the P.M (Figure S107) but did appear in the spectrum of HP- $\beta-C D \cdot 2$ (Figure S108). A number of bands attributed to guest molecule 2 were visible in the spectrum of HP- $\beta-C D \cdot 2$. Correlating stretches present in both the spectra of 2 and HP- $\beta-C D \cdot 2$ were found at 1563/1545 $\mathrm{cm}^{-1}$, $1509 / 1504 \mathrm{~cm}^{-1}, 1336 / 1324 \mathrm{~cm}^{-1}$, and $1224 / 1230 \mathrm{~cm}^{-1}$. The band at $1256 \mathrm{~cm}^{-1}$ was present in the spectrum of the P.M but disappeared entirely in the spectrum of HP- $\beta-C D \cdot 2$ (Figure S108), suggesting that the formation of the inclusion complex inhibits vibration of the $\mathrm{C}=\mathrm{S}$ bond. The inclusion of the adamantyl group in the HP- $\beta-C D$ cavity is more likely to affect the vibration of the $\mathrm{C}=\mathrm{S}$ bond than the aromatic $\mathrm{C}-\mathrm{H}$ bonds because the thiourea is located closer to the adamantyl moiety. In the IR spectrum of $\mathrm{HP}-\beta-\mathrm{CD} \cdot \mathbf{4}$, peaks attributed to compound 4 are visible at 2902, 1667 and $1503 \mathrm{~cm}^{-1}$. There is a small upfield shift compared to the corresponding peaks in the spectrum of the free compound. In the spectrum of HP- $\beta-C D \cdot 6$, only peaks at 1415,1331 and $850 \mathrm{~cm}^{-1}$ can be attributed to the transporter molecule.

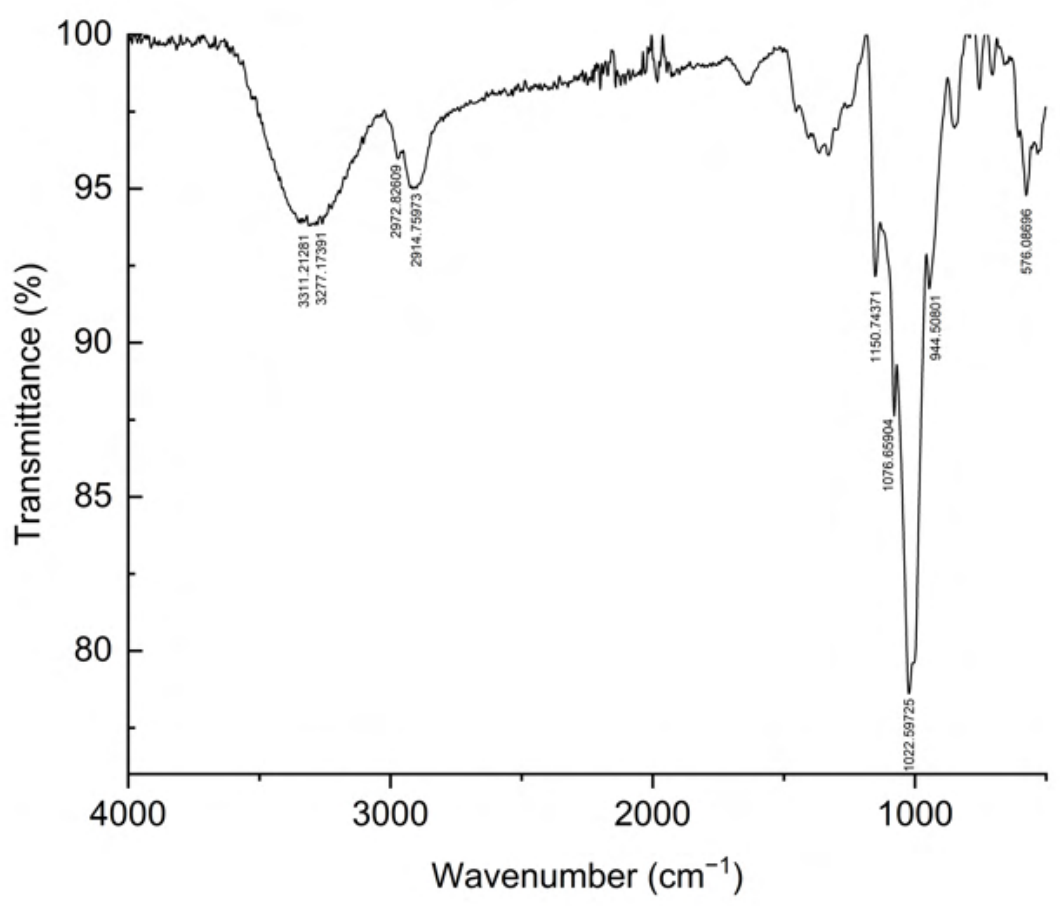

Figure S101. FTIR spectrum of HP- $\beta-C D$. 


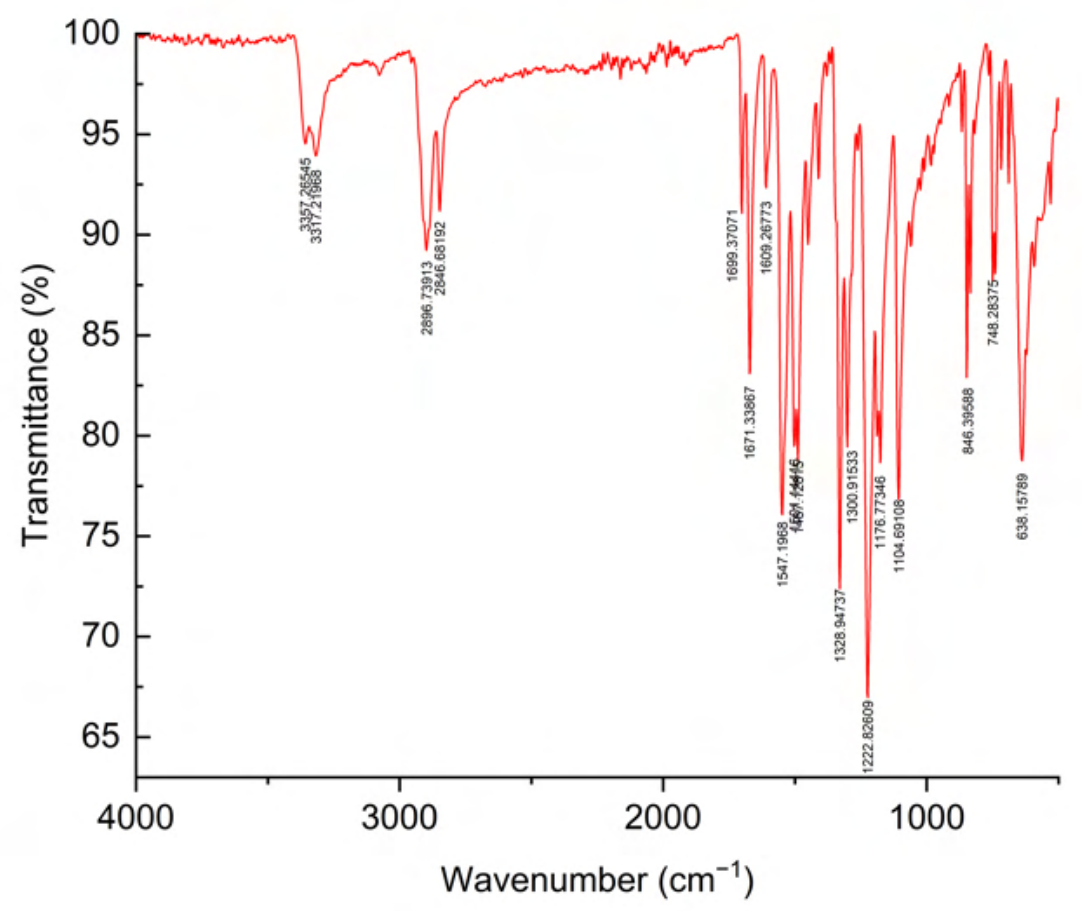

Figure S102. FTIR spectrum of compound 1.

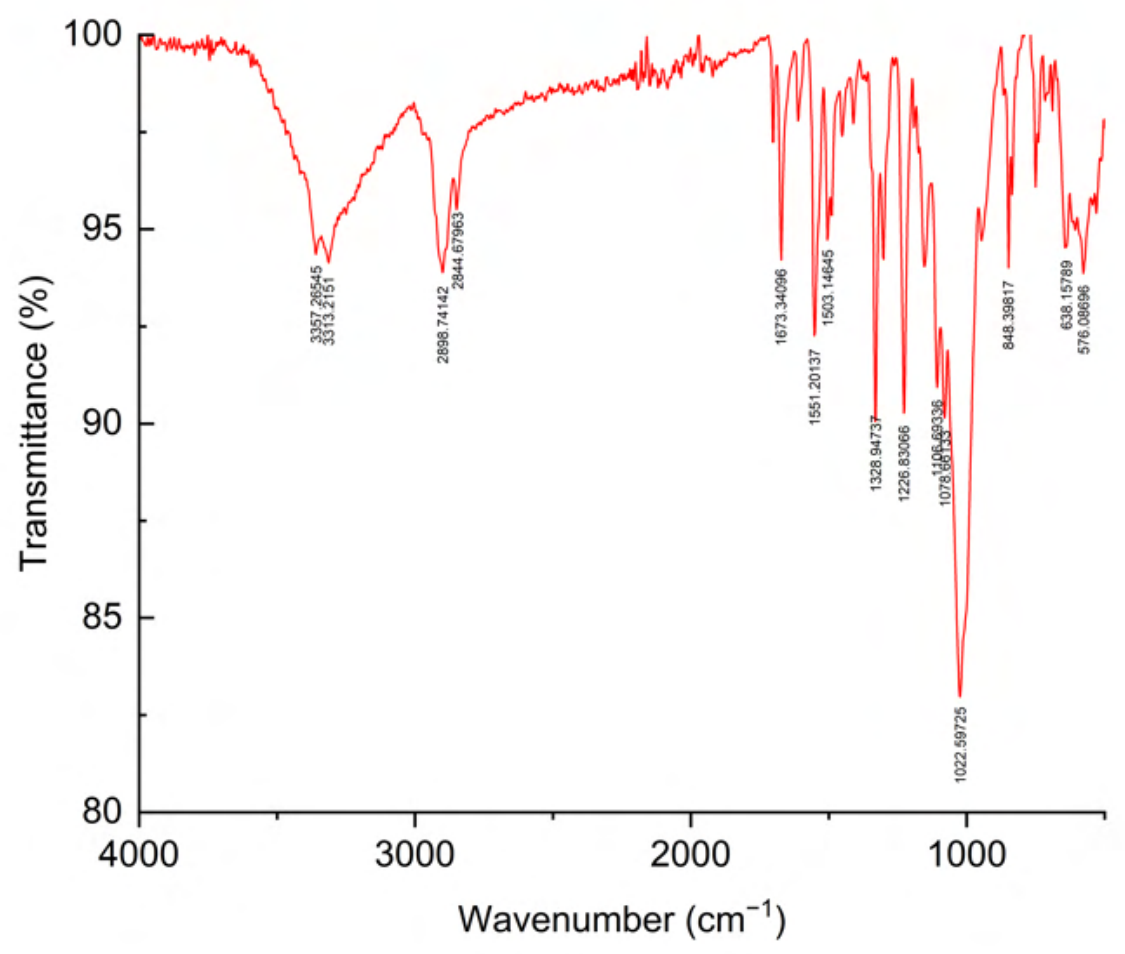

Figure S103. FTIR spectrum of an equimolar physical mixture of HP- $\beta-C D$ and compound 1. 


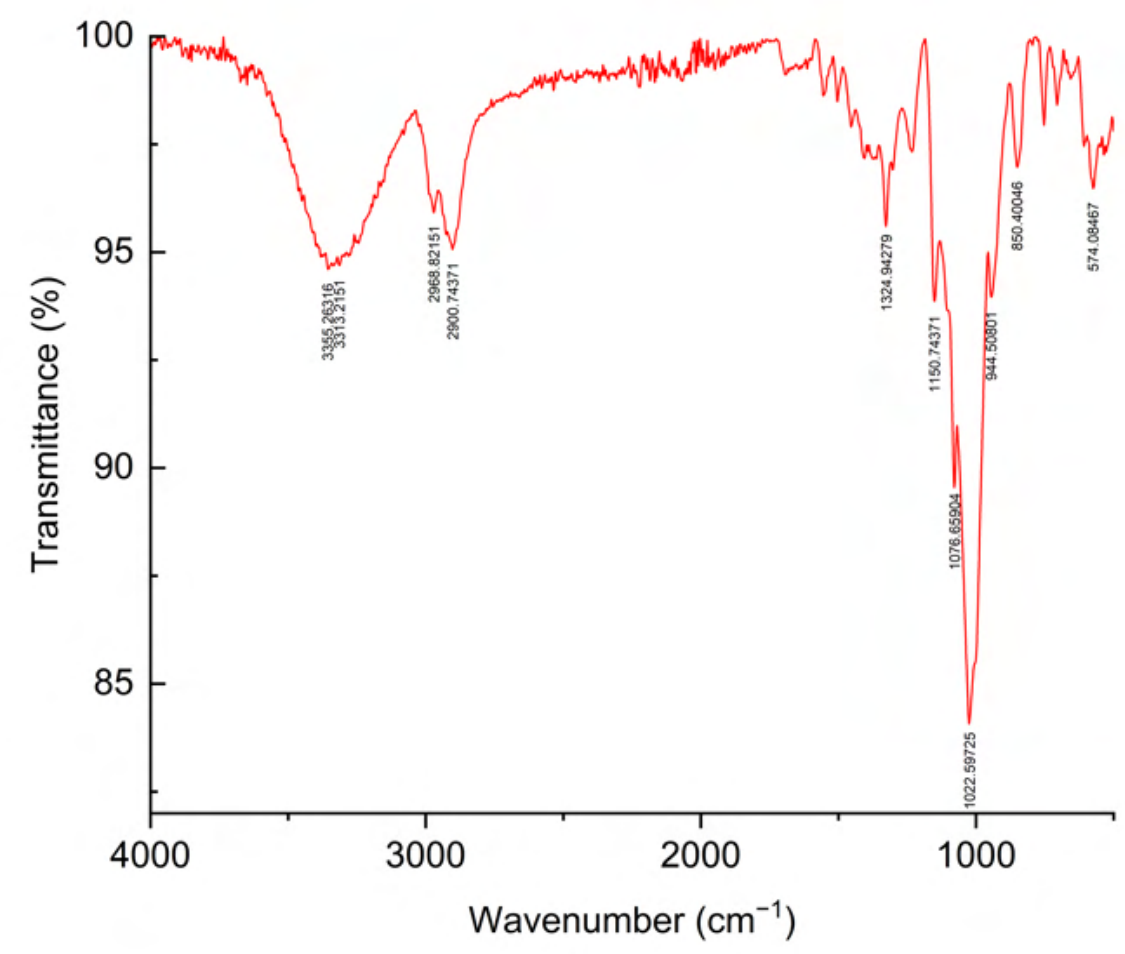

Figure S104. FTIR spectrum of HP- $\beta-C D \cdot 1$. 


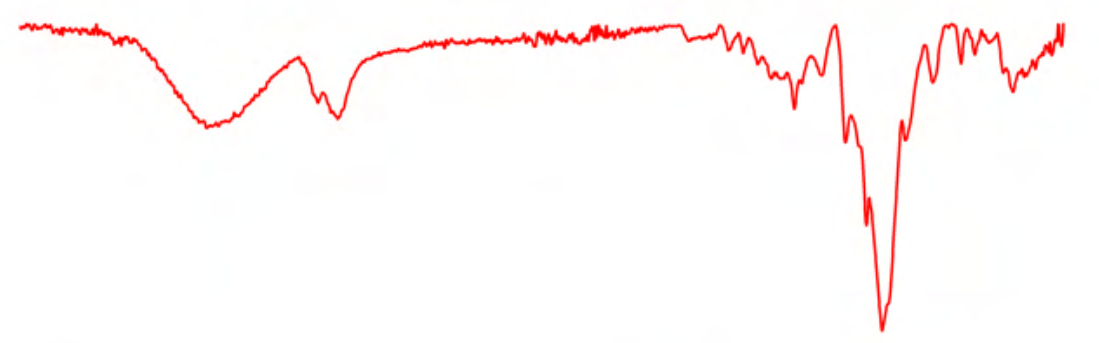

d)

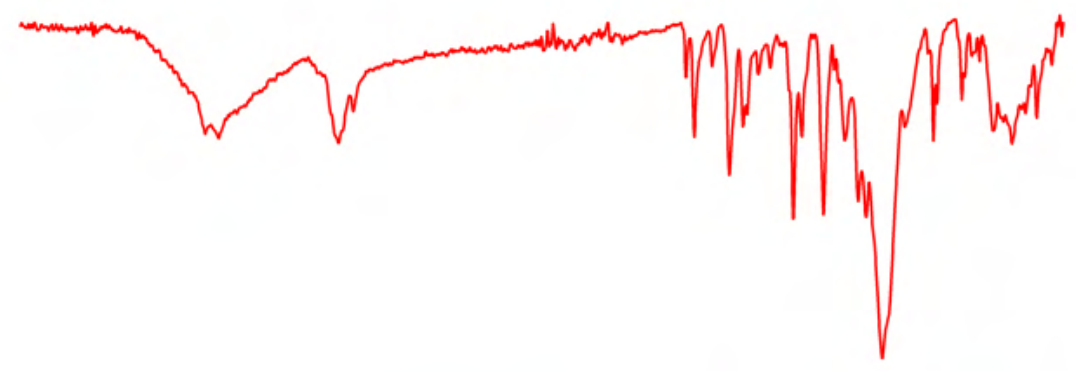

c)

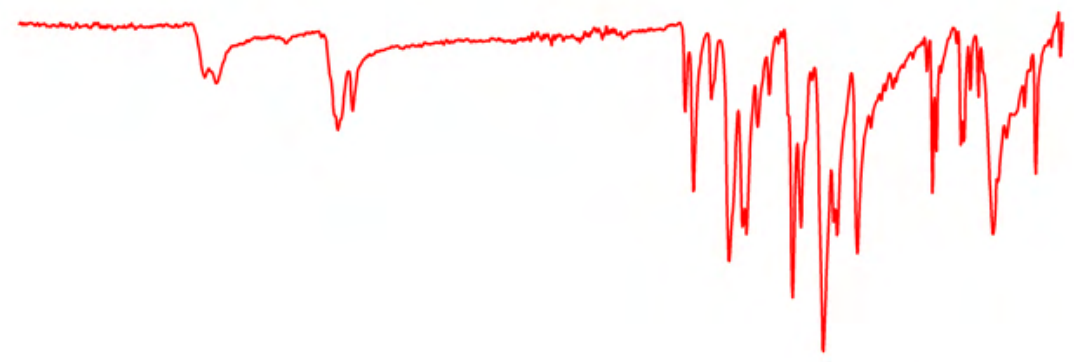

b)

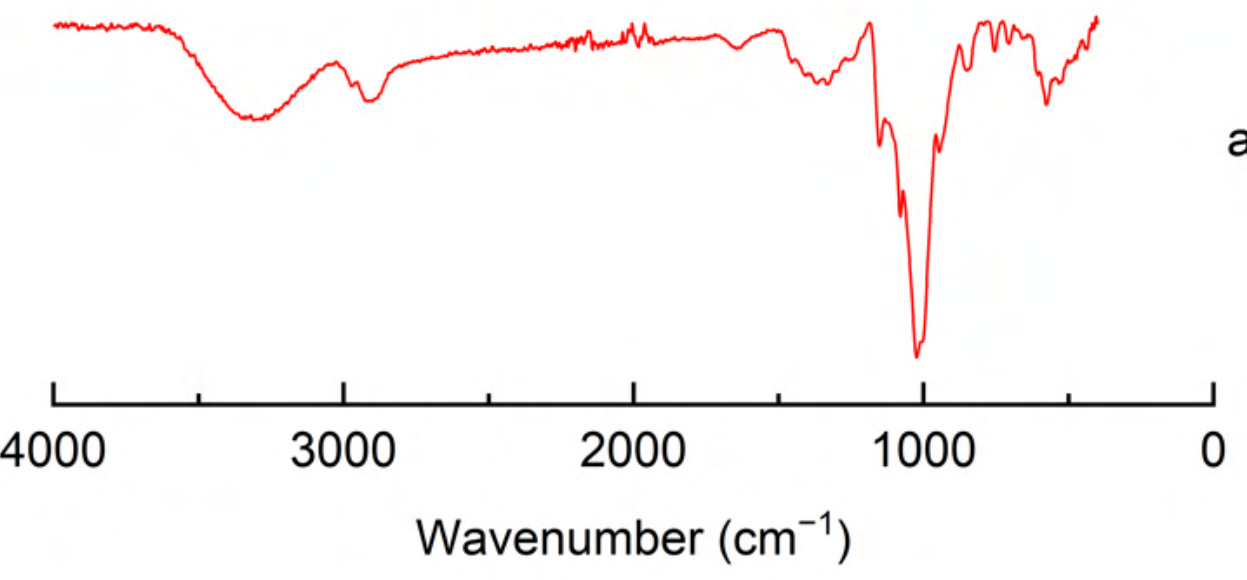

Figure S105. ATR-FTIR spectrum of (a) HP- $\beta$-CD, (b) 1, (c) physical mixture of HP- $\beta$-CD and 1, (d) inclusion complex HP- $\beta$-CD·1. 


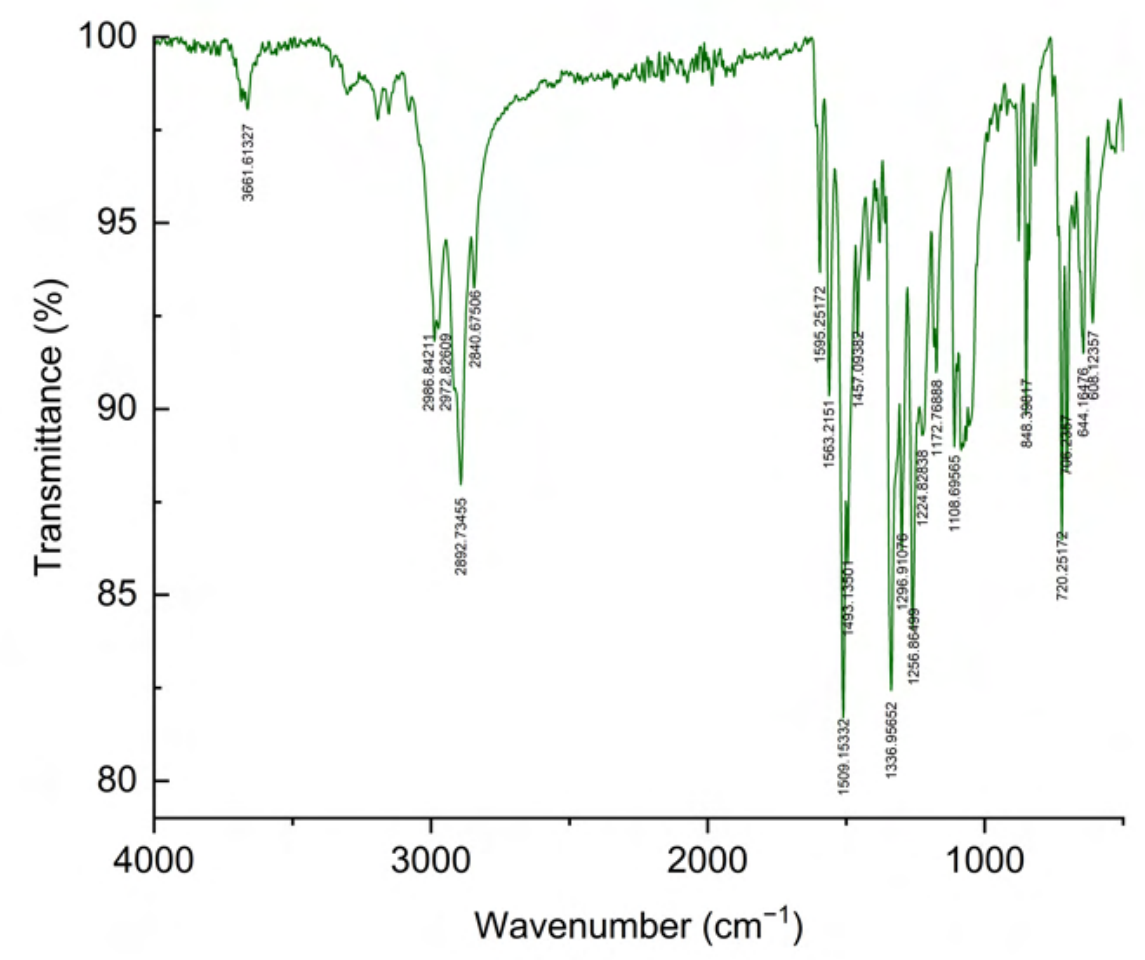

Figure S106. FTIR spectrum of compound 2.

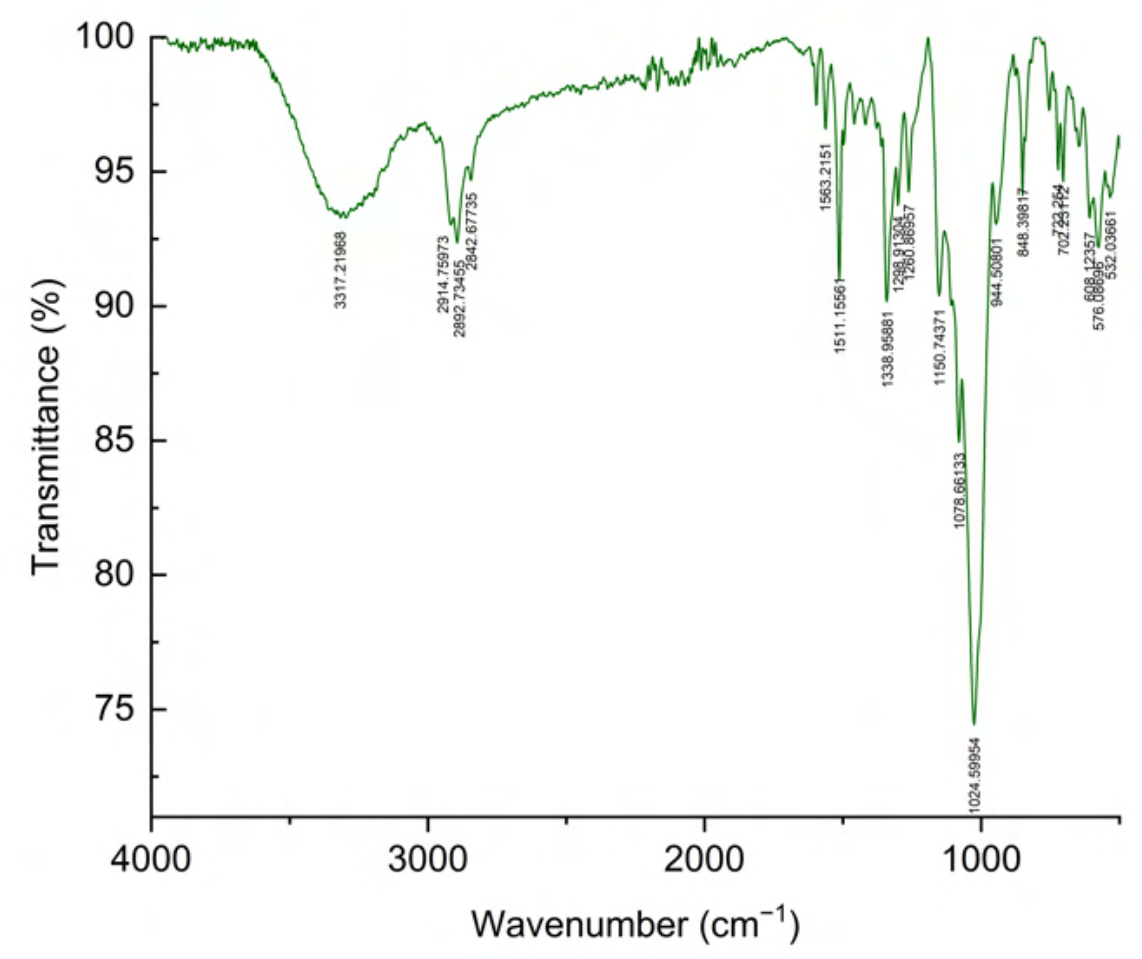

Figure S107. FTIR spectrum of an equimolar physical mixture of HP- $\beta$-CD and compound 2. 


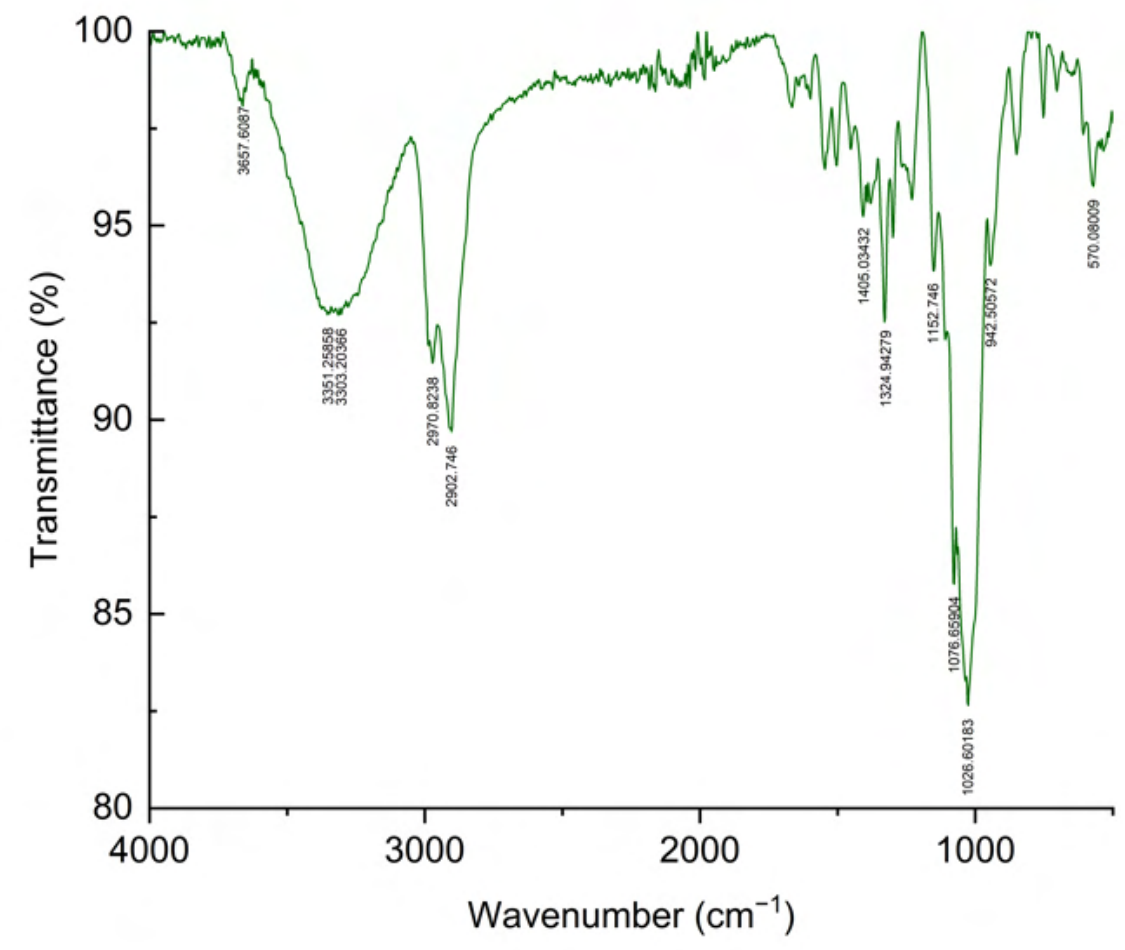

Figure S108. FTIR spectrum of HP- $\beta-C D \cdot 2$. 


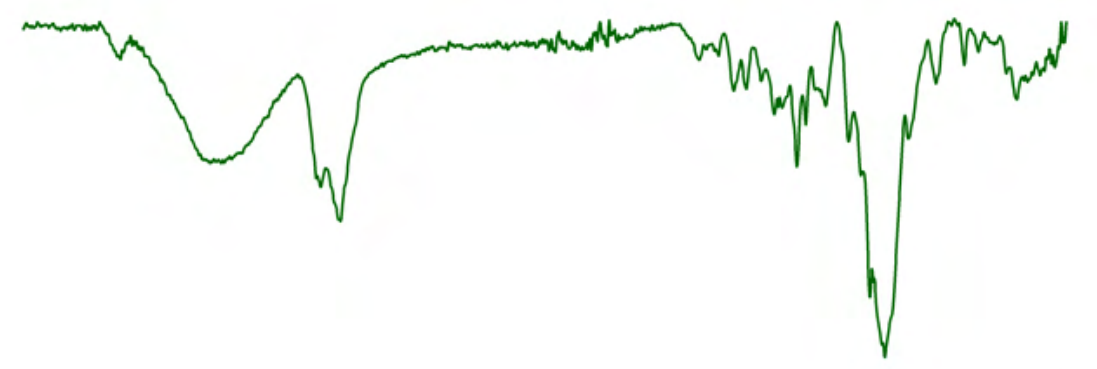

d)

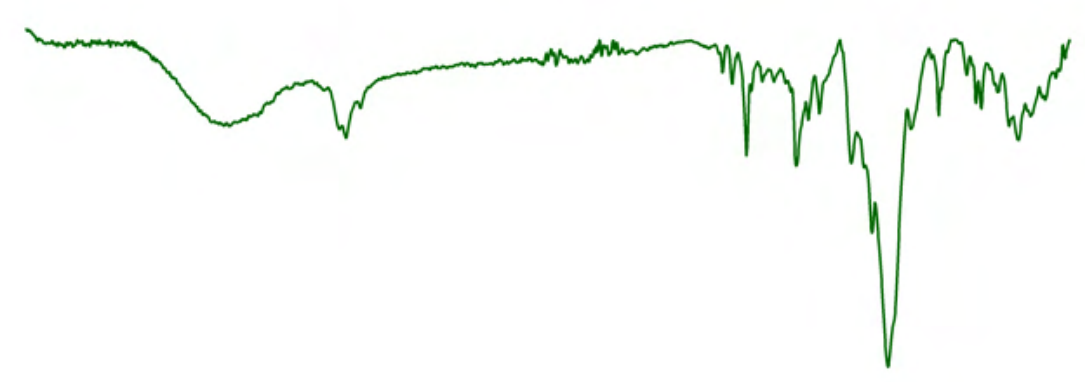

c)

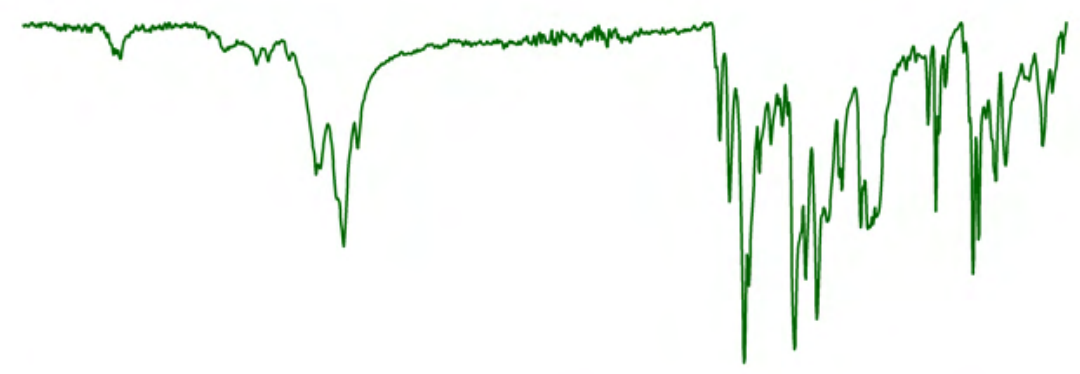

b)

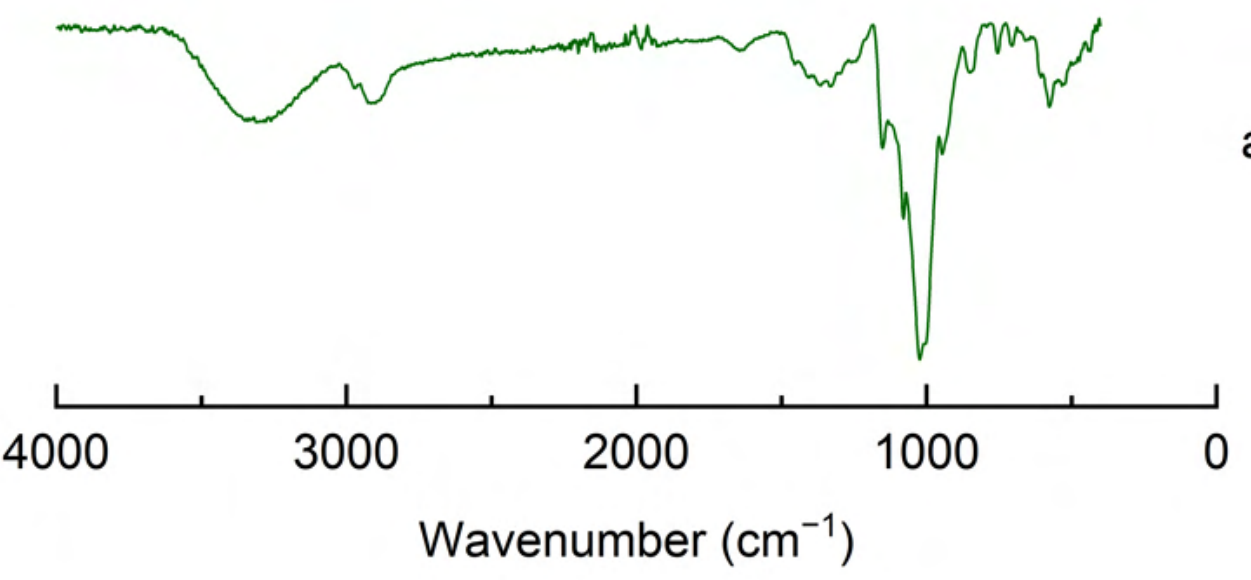

Figure S109. ATR-FTIR spectrum of (a) HP- $\beta$-CD, (b) 2, (c) physical mixture of HP- $\beta$-CD and 2, (d) inclusion complex HP- $\beta$-CD 2 . 


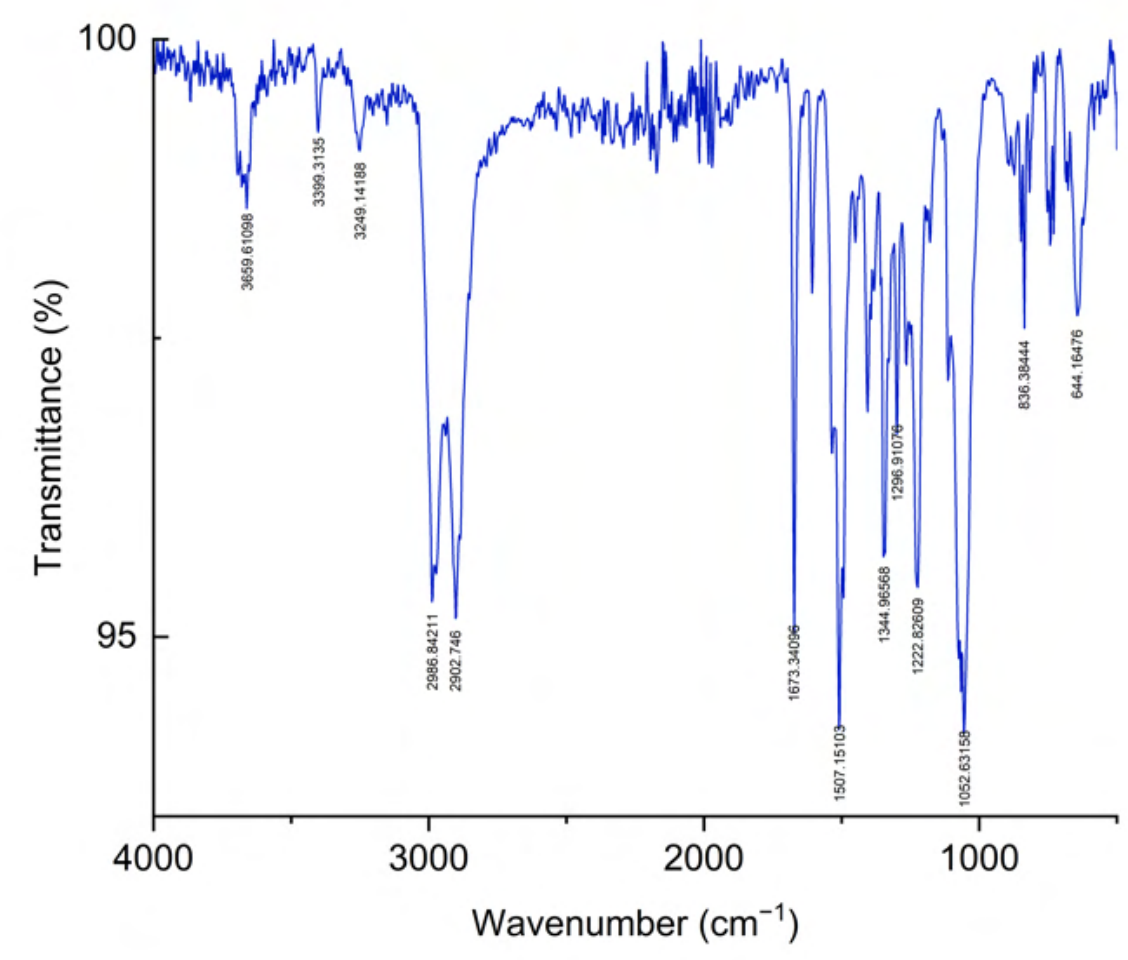

Figure S110. FTIR spectrum of compound 4.

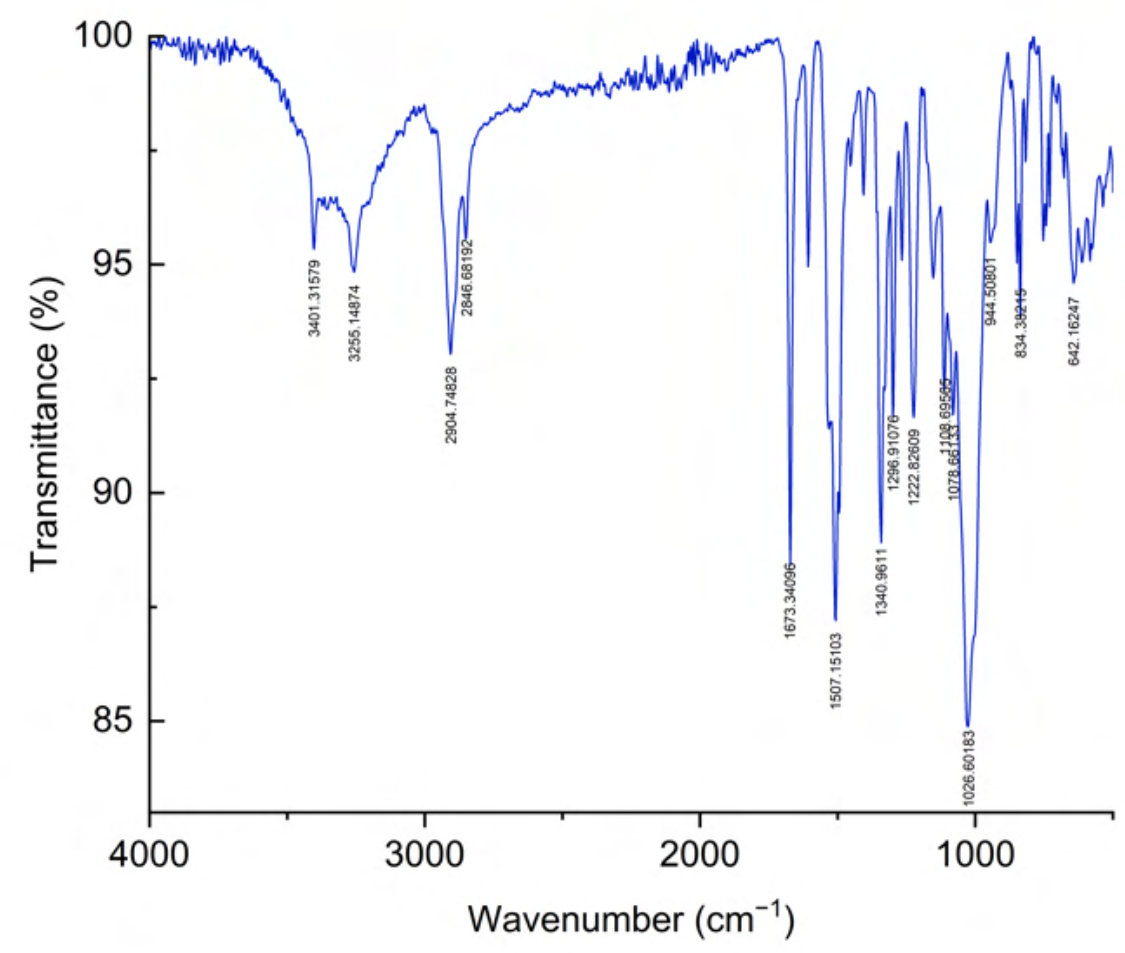

Figure S111. FTIR spectrum of an equimolar physical mixture of HP- $\beta$-CD and compound 4. 


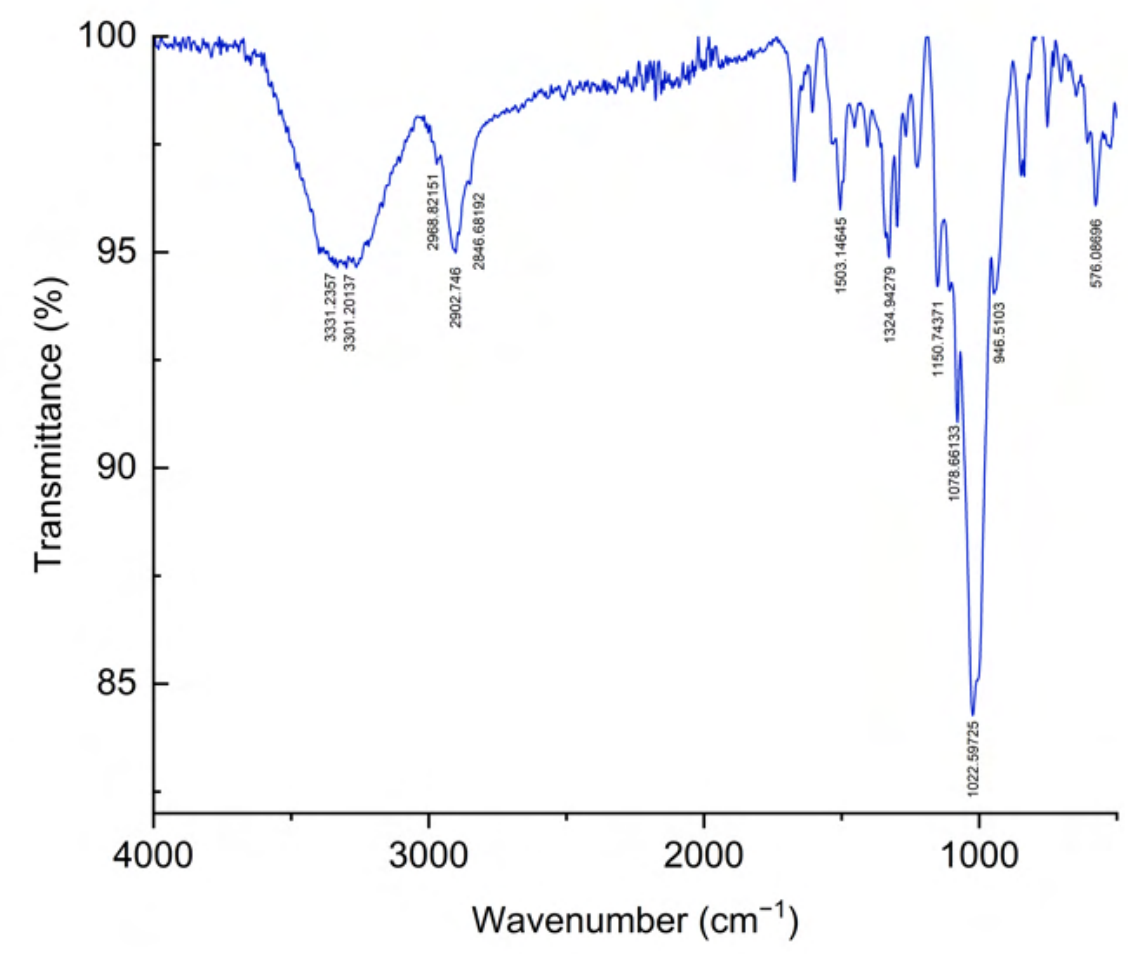

Figure S112. FTIR spectrum of HP- $\beta-C D \cdot 4$. 


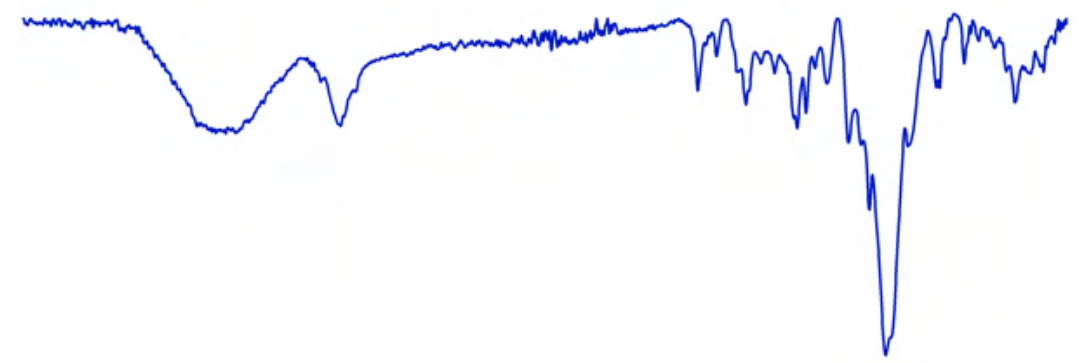

d)

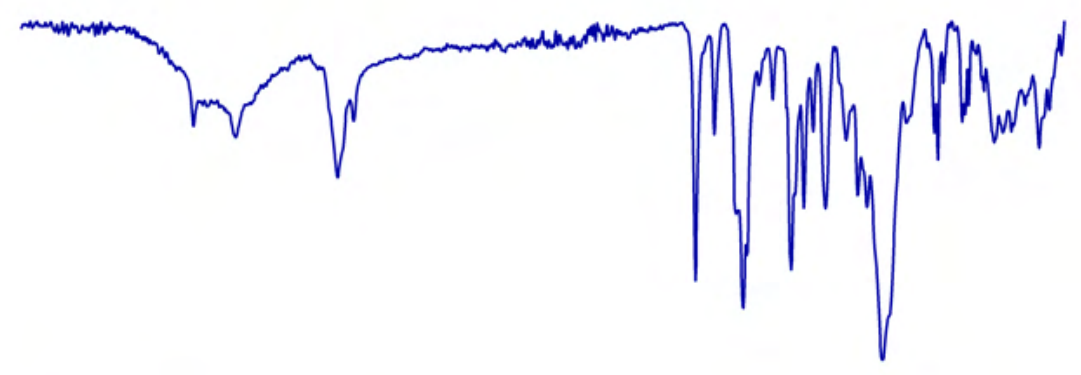

c)

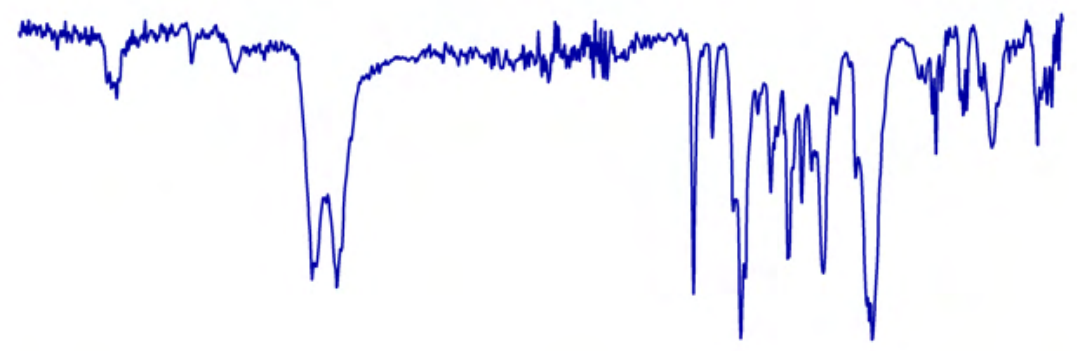

b)

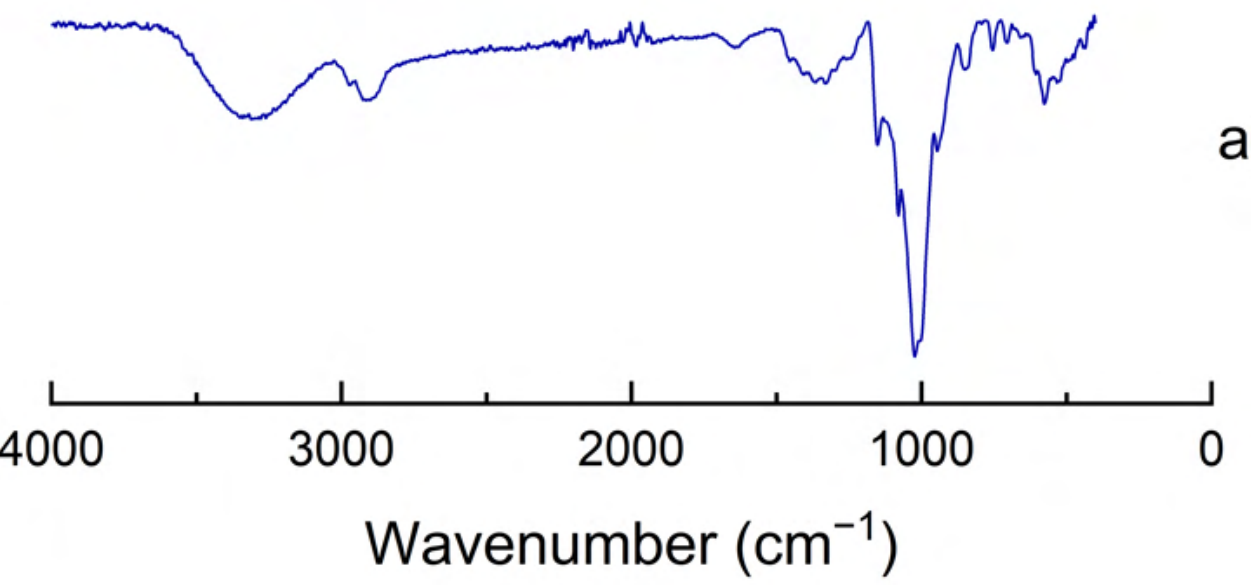

Figure S113. ATR-FTIR spectrum of (a) HP- $\beta-C D$, (b) 4, (c) physical mixture of HP- $\beta$-CD and 4, (d) inclusion complex HP- $\beta$-CD·4. 


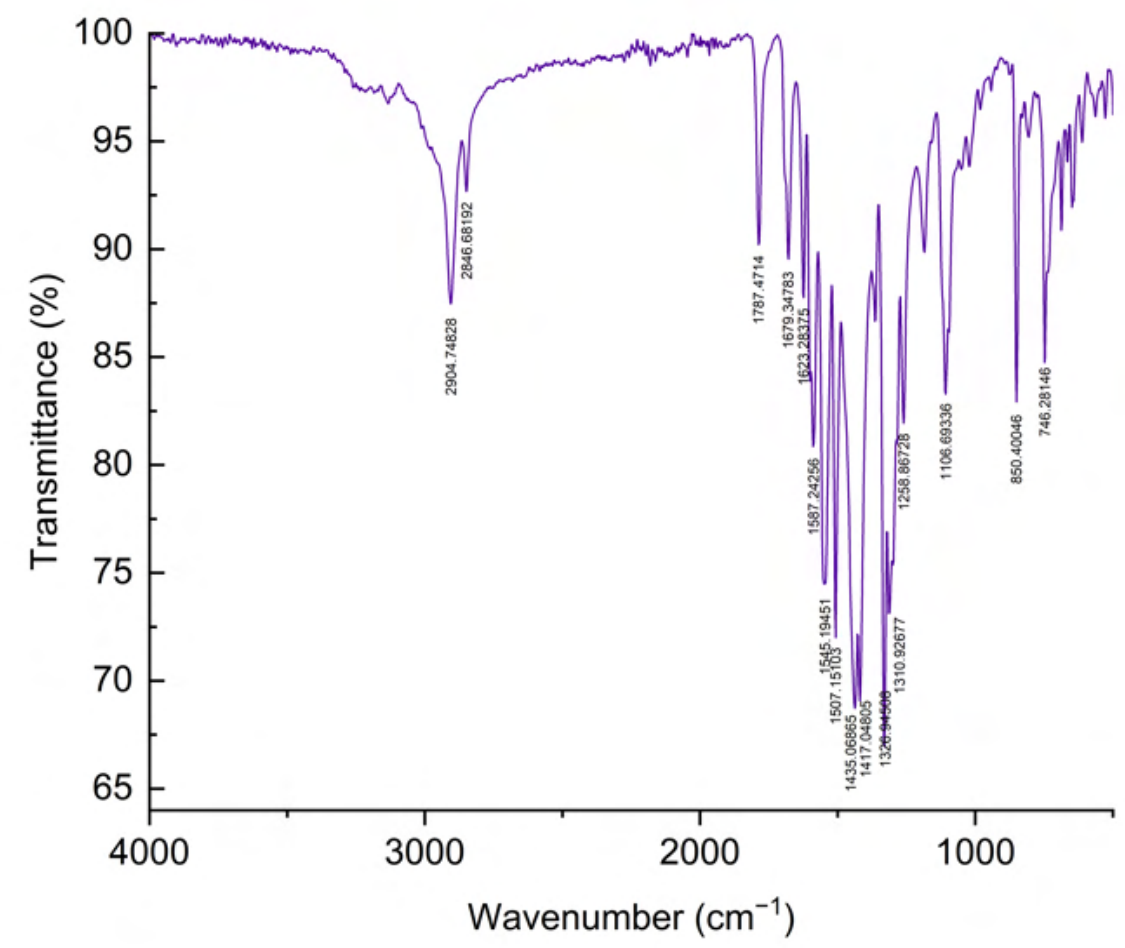

Figure S114. FTIR spectrum of compound 6.

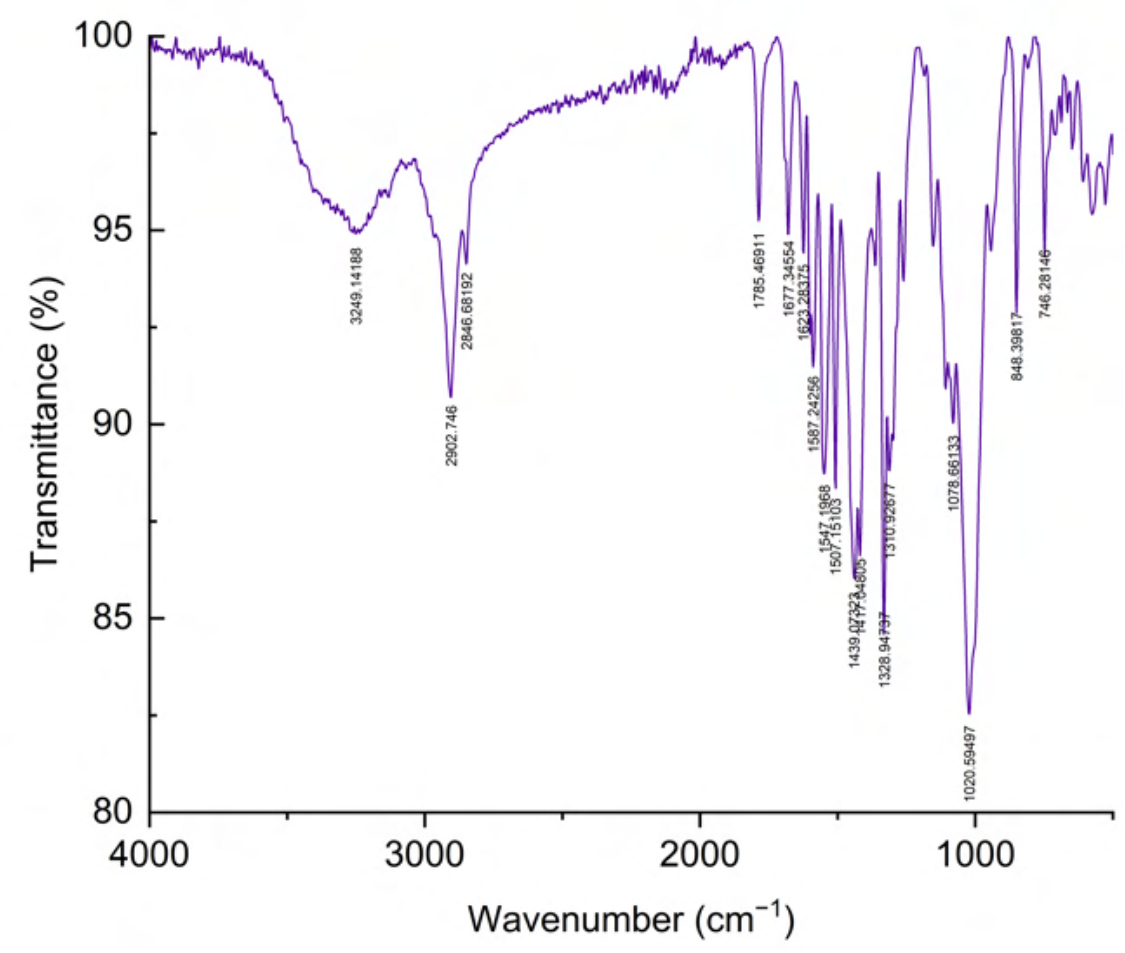

Figure S115. FTIR spectrum of an equimolar physical mixture of HP- $\beta$-CD and compound 6. 


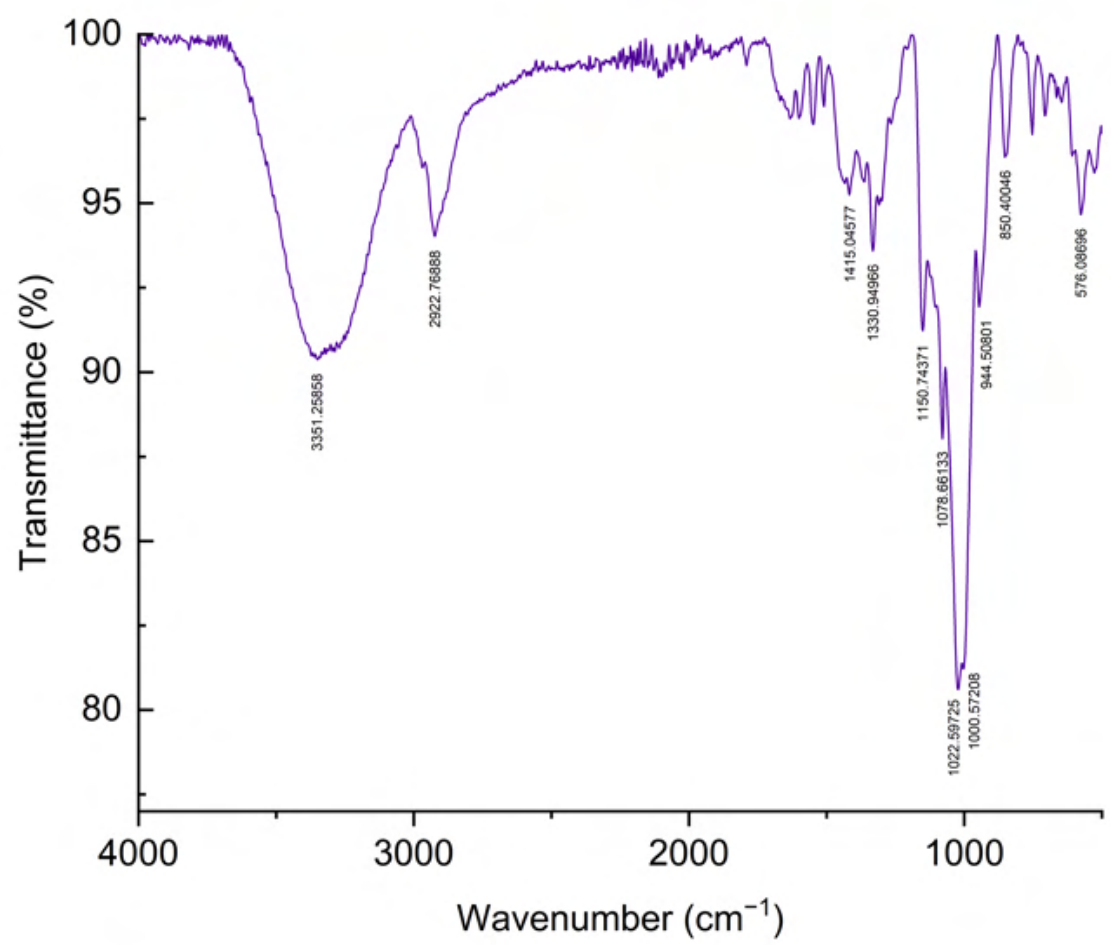

Figure S116. FTIR spectrum of HP- $\beta-C D \cdot 6$. 


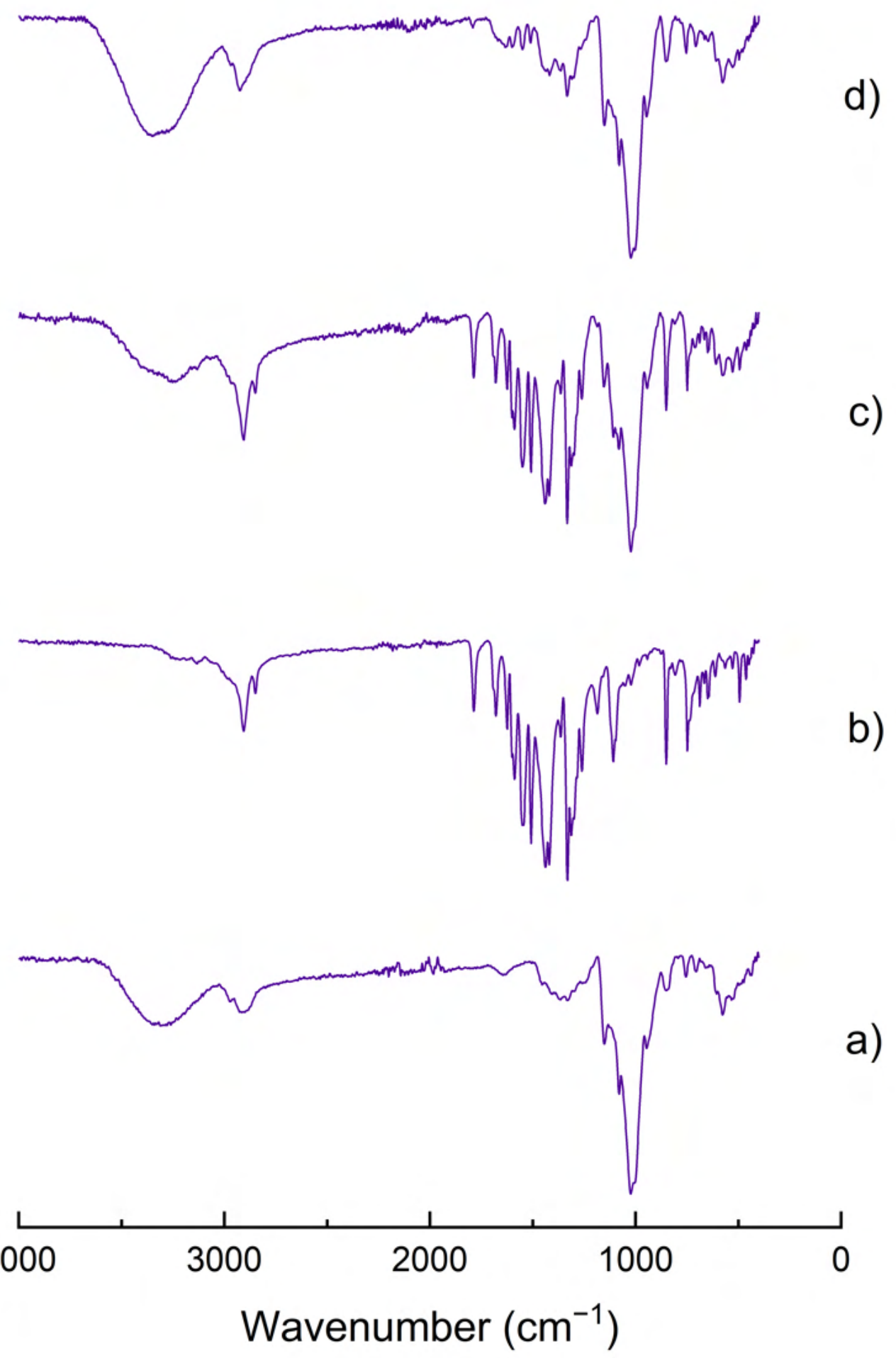

Figure S117. ATR-FTIR spectrum of a) HP- $\beta-C D, b) 6$, c) physical mixture of $\mathrm{HP}-\beta-\mathrm{CD}$ and 6, d) inclusion complex HP- $\beta-C D \cdot 6$. 


\section{2D-NOESY ${ }^{1} \mathrm{H}$ NMR Spectroscopic Analysis}

Two-dimensional NOESY ${ }^{1} \mathrm{H}$ NMR experiments were performed on the four inclusion complexes to investigate the spatial proximity of the HP- $\beta-\mathrm{CD}$ and transporter molecule hydrogens. The Nuclear Overhauser Effect (NOE) is the perturbation in intensity of an NMR resonance when a nearby proton is irradiated and occurs as a result of dipole-dipole crossrelaxation between the nuclei. ${ }^{25}$ The effect is not governed by the through-bond relationship of the two nuclei and, instead, is a purely though-space interaction. The strength of the NOE is dependent on proximity and is typically observed between protons that are up to $4 \AA$ apart. The appearance in the 2D-NOESY spectra of the inclusion complexes of NOE cross-peaks between resonances attributed with $\mathrm{HP}-\beta-\mathrm{CD}$ and resonances attributed to the guest molecule would indicate that nuclei of the two species are close in space. The most likely reason for this occurrence is the presence of an inclusion complex.

The partial contour plot for the 2D NOESY spectrum of HP- $\beta-C D \cdot \mathbf{1}$ can be seen in Figure S118. An appreciable correlation is seen between the signals attributed to the adamantyl protons of 1 and two of the signals relating to the protons of $\mathrm{HP}-\beta-\mathrm{CD}$, which are highlighted in Figure S119. On closer inspection (Figure S120), the cross-peaks can be attributed to the $\mathrm{H}^{3}$ and $\mathrm{H}^{5}$ protons of the macrocycle, which are located inside the HP- $\beta-C D$ cavity (Figure S44). The proximity in space of the adamantyl protons and the protons of the interior surface of HP- $\beta$ $\mathrm{CD}$ strongly suggest that an inclusion complex has formed between the two components. This is complemented by the absence of cross-peak correlations associated with other HP- $\beta-C D$ protons located on the outer surface of the structure $\left(\mathrm{H}^{1}, \mathrm{H}^{2}\right.$ and $\left.\mathrm{H}^{4}\right)$. The 2D NOESY spectra of HP- $\beta$-CD 2 and HP- $\beta-C D \cdot 6$ include similar cross-peak interactions between the adamantyl proton signals and the $\mathrm{H}^{3} / \mathrm{H}^{5}$ protons of $\mathrm{HP}-\beta-\mathrm{CD}$.

The cross-peak interactions present in the 2D NOESY spectrum of HP- $\beta-C D \cdot 4$ are less clear (Figure A.360), but seem to suggest an NOE interaction occurs between the adamantyl protons and the $\mathrm{H}^{5} / \mathrm{H}^{6}$ protons of $\mathrm{HP}-\beta-\mathrm{CD}$. Cross-peak interactions with the $\mathrm{H}^{3}$ protons are not visible. Compound 22 lacks a methyl spacer group, meaning the proximity of the urea moiety to the adamantyl group may limit the penetration of the transporter into the cavity if it enters from the narrower primary rim. The adamantyl is a better size match for the opening on the primary rim, so partial inclusion from this side would be more favourable. This would 
explain the through-space interaction between the adamantyl protons and the $\mathrm{H}^{6}$ protons of $\mathrm{HP}-\beta-\mathrm{CD}$, which are also located on the primary side of the structure.

Interestingly, cross-peak interactions were also visible between the $\mathrm{H}^{3} / \mathrm{H}^{5}$ protons, and the signals related to the aryl protons of the guest molecules. This was unexpected, as the compounds were designed with the intention that the adamantyl moiety would enter the cyclodextrin cavity. Several possibilities could explain the existence of NOE interactions between the aryl transporter protons and the interior cavity protons of HP- $\beta-\mathrm{CD}$. Firstly, it could be that the adamantane sits in the primary rim of HP- $\beta-C D$ whilst the remainder of the molecule penetrates the cavity. This would agree with the perfect size match that exists between adamantyl groups and $\beta$-CD derivatives, which other researchers have exploited. A second possibility is that the 1:1 host:guest complex is unselective, and either the $p$ nitrophenol group or the adamantyl moiety penetrates the cavity to form the inclusion complex. Finally, it may suggest that both ends of the transporter molecule are partially included inside a cyclodextrin molecule, forming a 2:1 host:guest complex. This theory would offer an explanation for the apparent HP- $\beta$-CD:transporter stoichiometries, which included more $\mathrm{HP}-\beta-\mathrm{CD}$ than expected because the two components were combined during inclusion complex formation in equimolar amounts. 


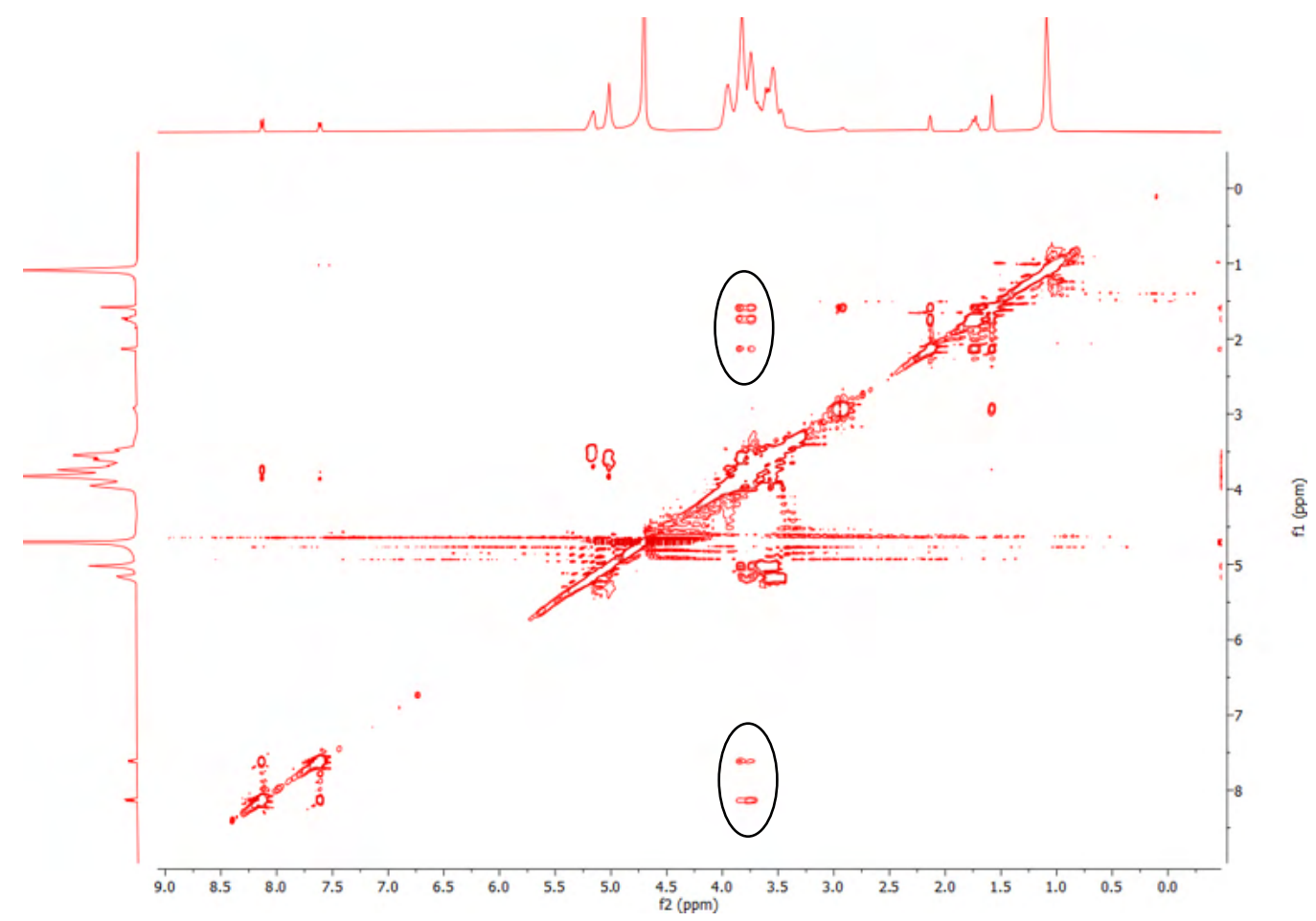

Figure S118. The 2D NOESY ${ }^{1} \mathrm{H}-\mathrm{NMR}$ spectrum of $\mathrm{HP}-\beta-\mathrm{CD} \cdot 1$ complex, in $\mathrm{D}_{2} \mathrm{O}$. Intermolecular cross-peak interactions between HP- $\beta-C D$ and 1 have been circled.

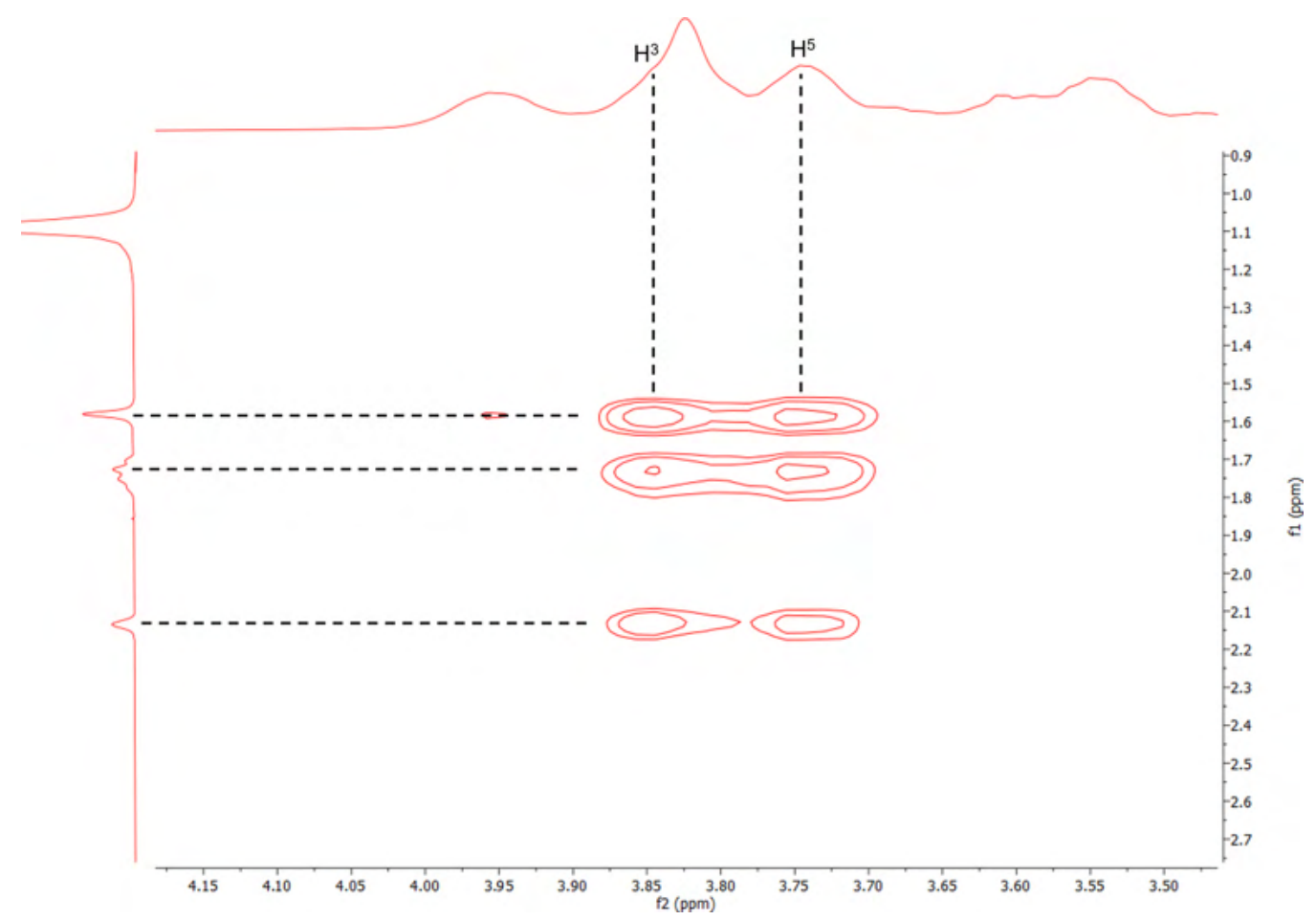

Figure S119. A magnified segment of the 2D NOESY ${ }^{1} \mathrm{H}-\mathrm{NMR}$ spectrum of the HP- $\beta-\mathrm{CD} \cdot \mathbf{1}$ complex, showing the cross-peak interactions between the signals of the adamantyl protons of $\mathbf{1}(\delta=1.58,1.73$, and $2.13 \mathrm{ppm})$ and the $\mathrm{H}^{3}$ and $\mathrm{H}^{5}$ protons of $\mathrm{HP}-\beta-\mathrm{CD}(\delta=3.85 \mathrm{ppm}$ and $\delta$ $=3.75$ ppm, respectively). 


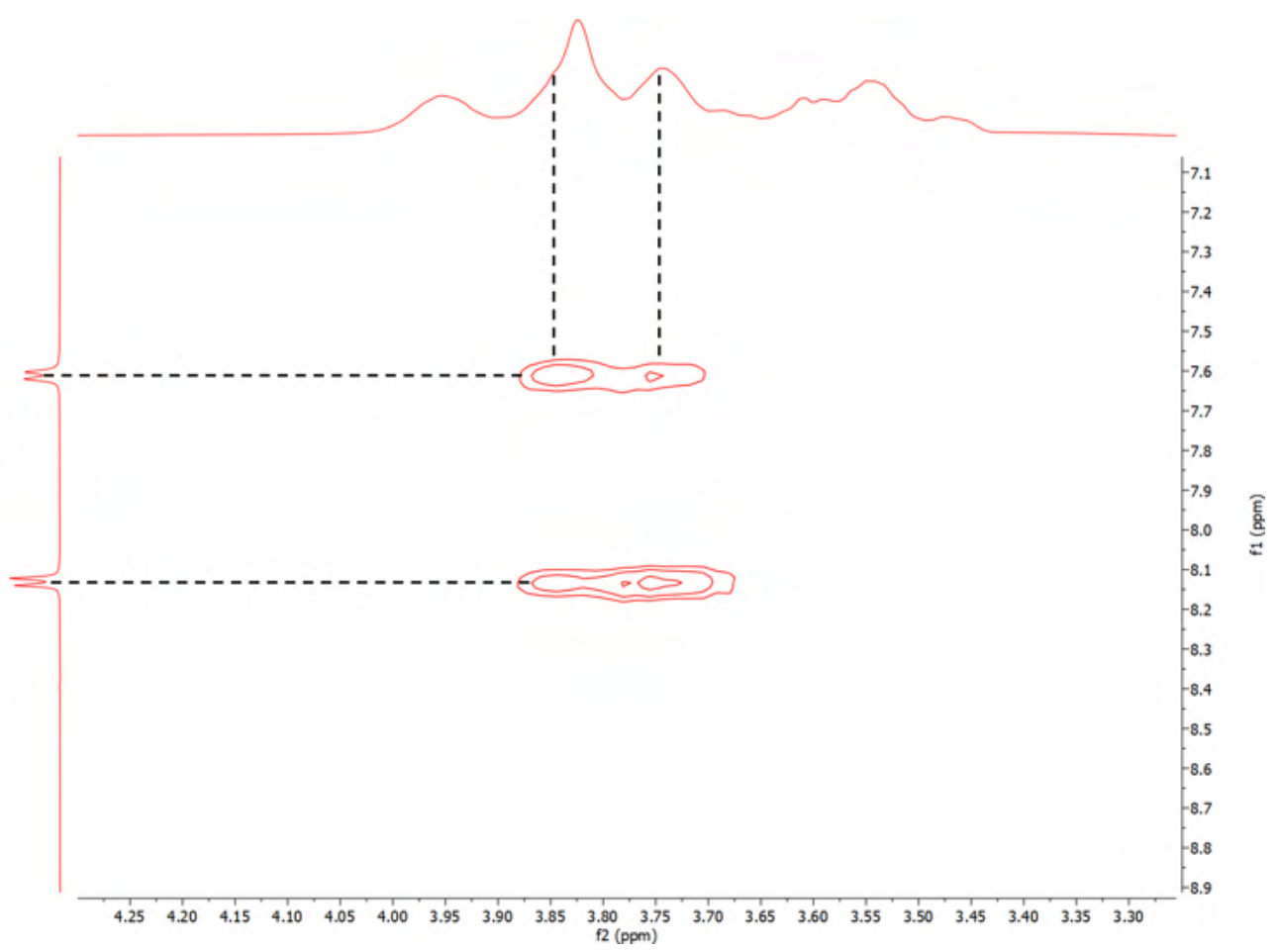

Figure S120. A magnified segment of the $2 \mathrm{D}$ NOESY ${ }^{1} \mathrm{H}-\mathrm{NMR}$ spectrum of the HP- $\beta-\mathrm{CD} \cdot \mathbf{1}$ complex, showing the cross-peak interactions between the signals of the aromatic protons of $1(\delta=7.61$ and $8.13 \mathrm{ppm})$ and the $\mathrm{H}^{3}$ and $\mathrm{H}^{5}$ protons of $\mathrm{HP}-\beta-\mathrm{CD}(\delta=3.85 \mathrm{ppm}$ and $\delta=3.75$ ppm, respectively).

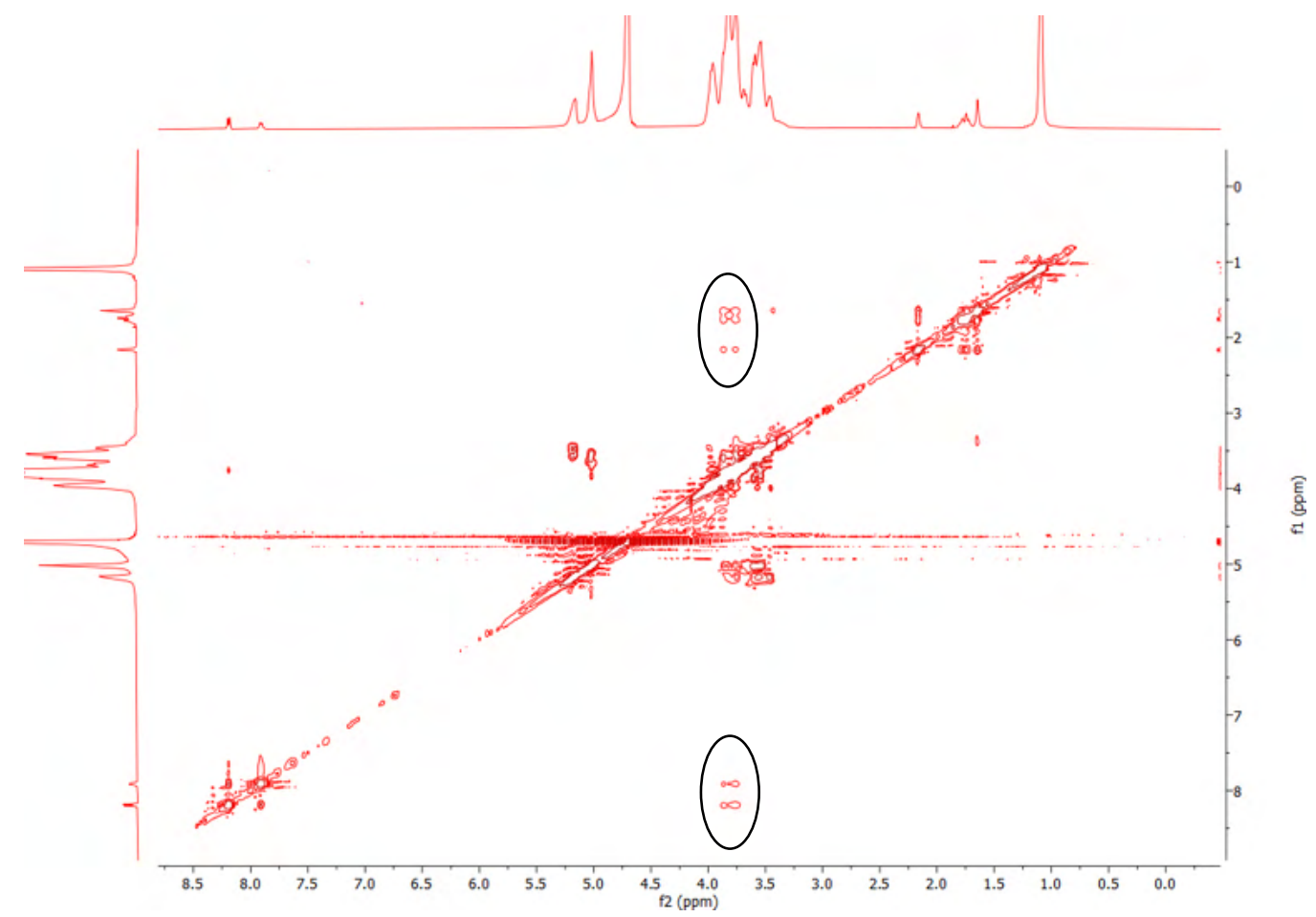

Figure S121. The 2D NOESY ${ }^{1} \mathrm{H}-\mathrm{NMR}$ spectrum of $\mathrm{HP}-\beta-\mathrm{CD} \cdot 2$ complex, in $\mathrm{D}_{2} \mathrm{O}$. Intermolecular cross-peak interactions between HP- $\beta-C D$ and 2 have been circled. 


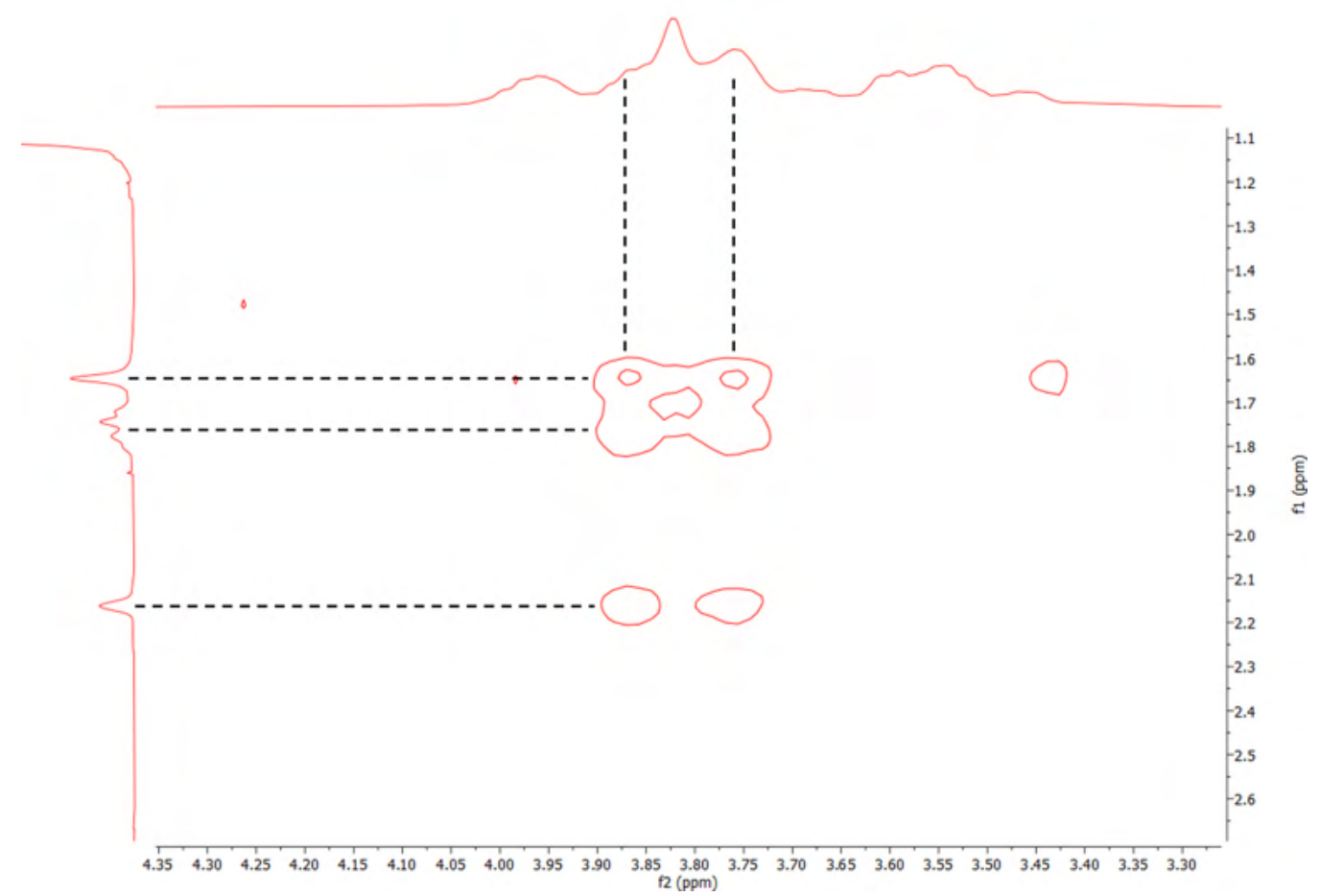

Figure S122. A magnified segment of the 2D NOESY ${ }^{1} \mathrm{H}-\mathrm{NMR}$ spectrum of the HP- $\beta-\mathrm{CD} \cdot 2$ complex, showing the cross-peak interactions between the signals of the adamantyl protons of $2(\delta=1.64,1.76$, and $2.16 \mathrm{ppm})$ and the $\mathrm{H}^{3}$ and $\mathrm{H}^{5}$ protons of $\mathrm{HP}-\beta-\mathrm{CD}(\delta=3.85 \mathrm{ppm}$ and $\delta$ $=3.75$ ppm, respectively).

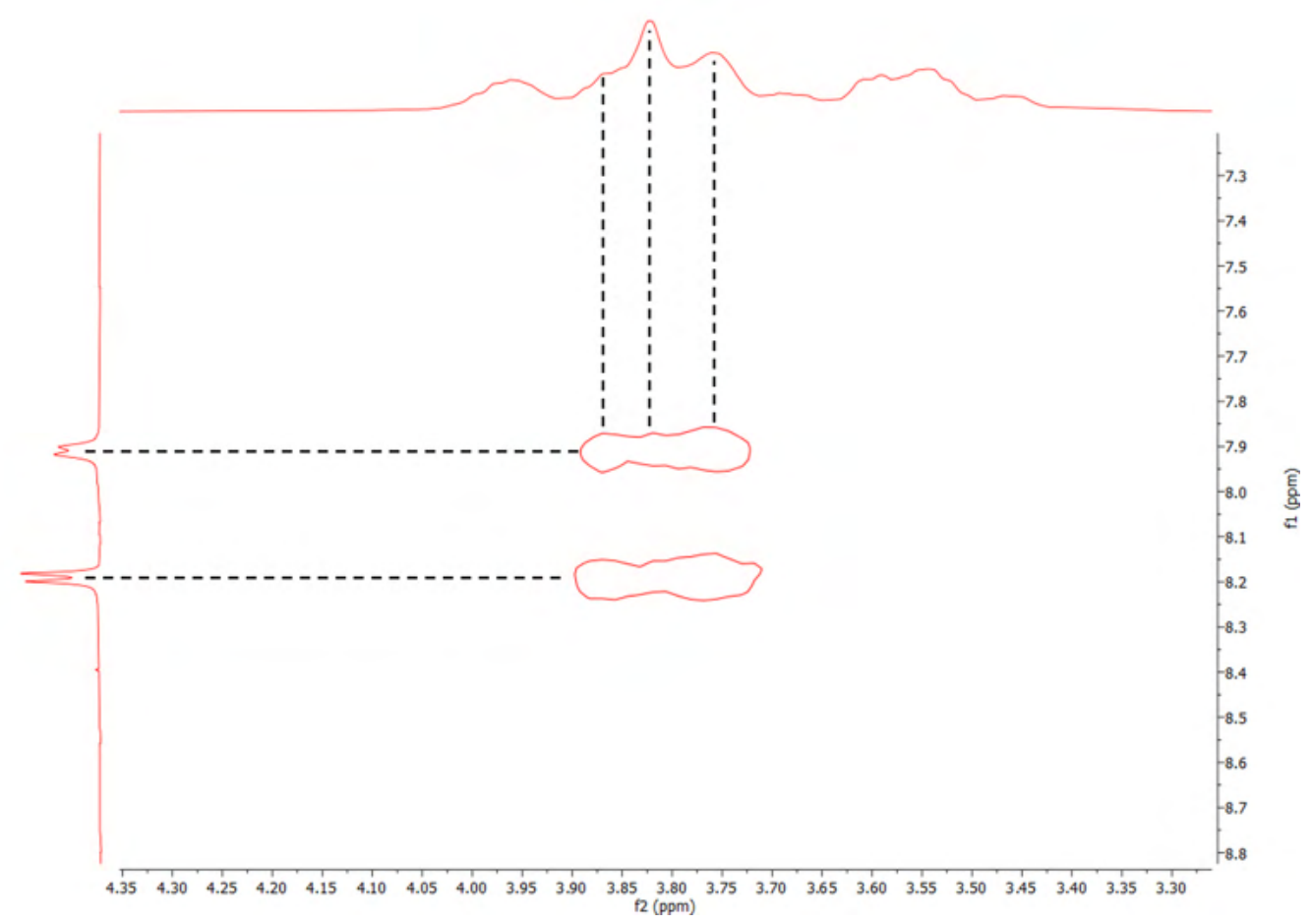

Figure S123. A magnified segment of the 2D NOESY ${ }^{1} \mathrm{H}-\mathrm{NMR}$ spectrum of the HP- $\beta-\mathrm{CD} \cdot 2$ complex, showing the cross-peak interactions between the signals of the aromatic protons of 
$2(\delta=7.91$ and $8.19 \mathrm{ppm})$ and the $\mathrm{H}^{3}, \mathrm{H}^{5}$ and $\mathrm{H}^{6}$ protons of $\mathrm{HP}-\beta-\mathrm{CD}(\delta=3.85 \mathrm{ppm}, \delta=3.75$ ppm, and $\delta=3.82$ ppm, respectively).

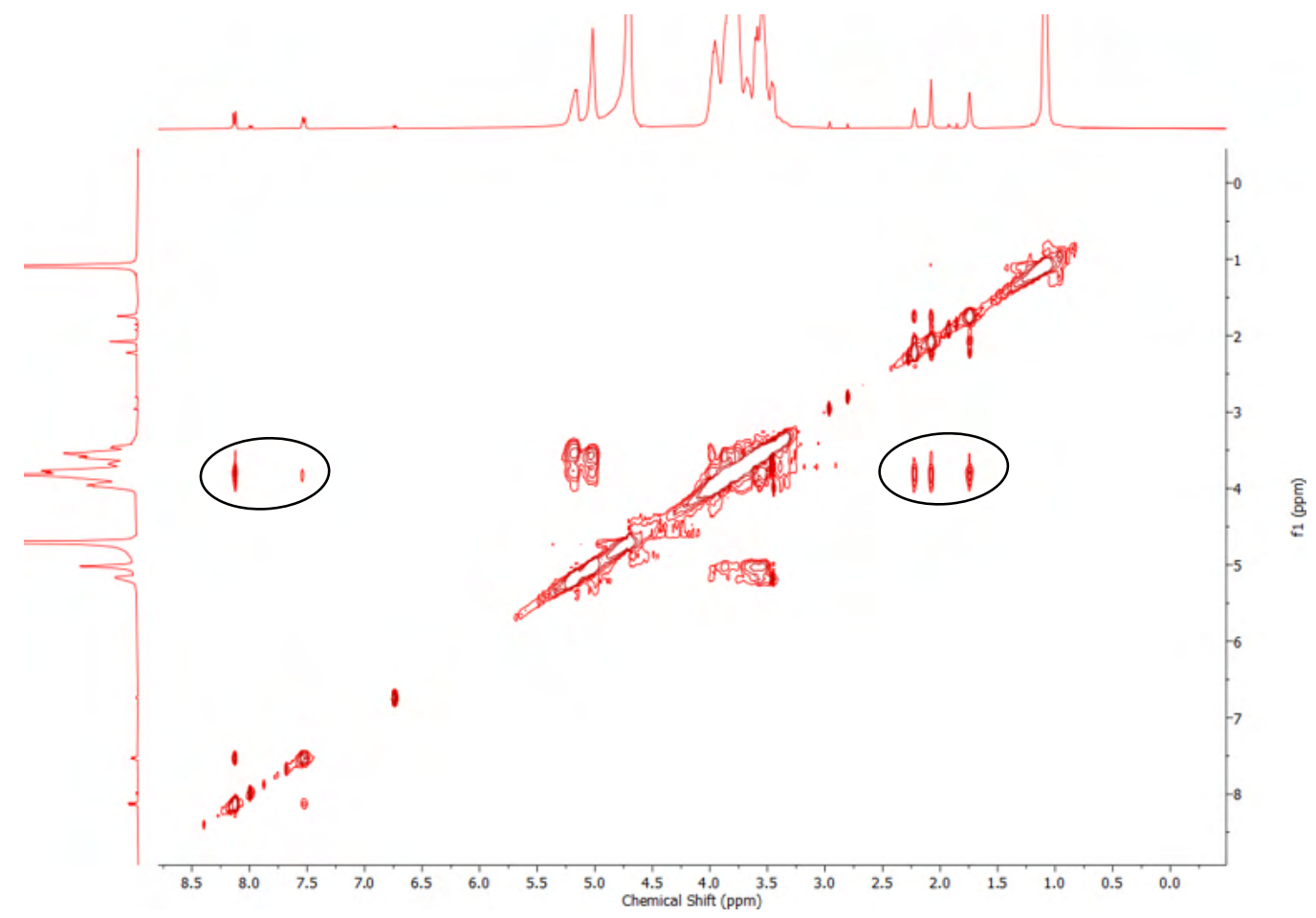

Figure S124. The 2D NOESY ${ }^{1} \mathrm{H}-\mathrm{NMR}$ spectrum of $\mathrm{HP}-\beta-\mathrm{CD} \cdot 4$ complex, in $\mathrm{D}_{2} \mathrm{O}$. Intermolecular cross-peak interactions between HP- $\beta-C D$ and 4 have been circled. 


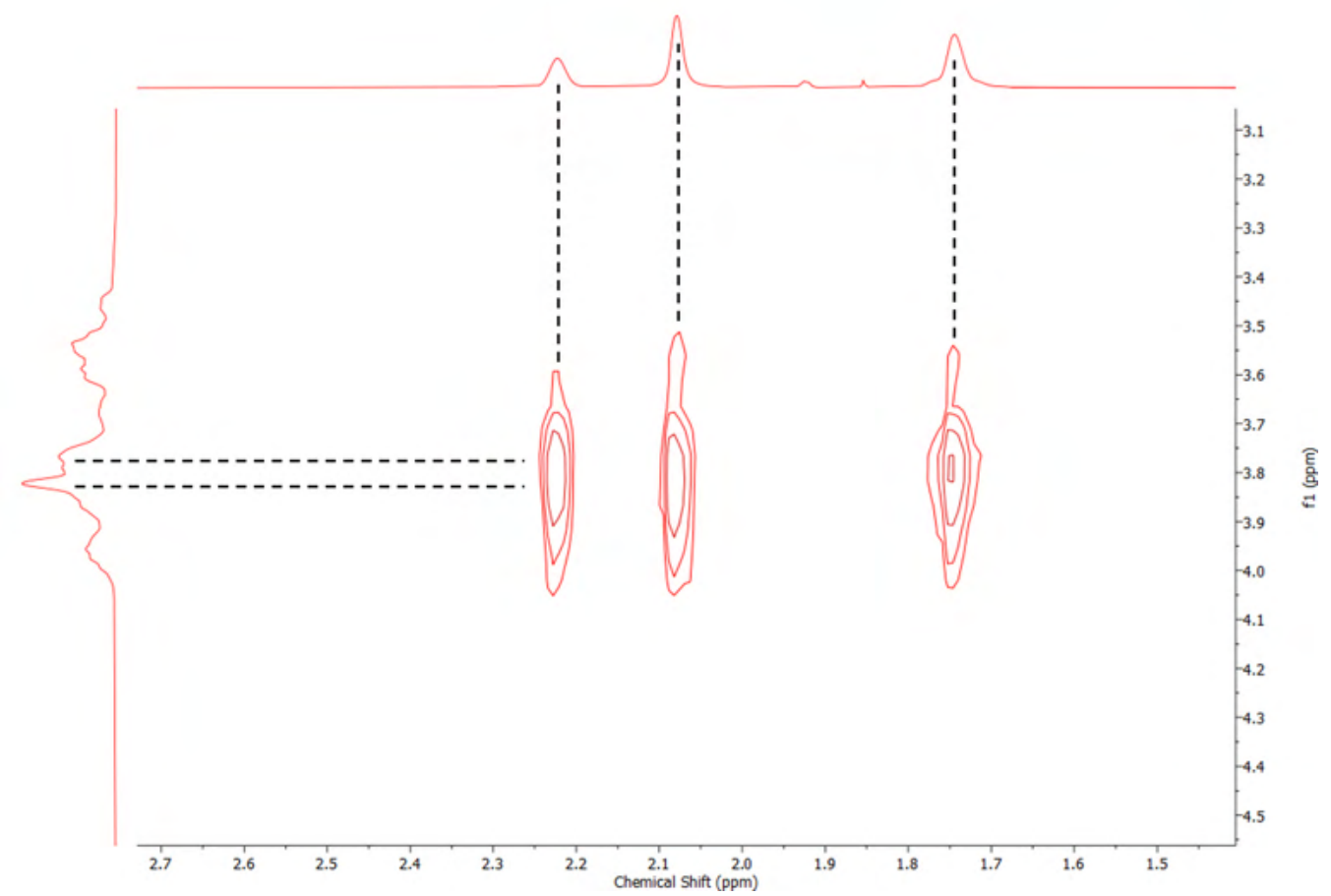

Figure S125. A magnified segment of the 2D NOESY ${ }^{1} \mathrm{H}-\mathrm{NMR}$ spectrum of the HP- $\beta-\mathrm{CD} \cdot 4$ complex, showing the cross-peak interactions between the signals of the adamantyl protons of $4(\delta=1.74,2.08$, and $2.22 \mathrm{ppm})$ and the $\mathrm{H}^{5}$ and $\mathrm{H}^{6}$ protons of $\mathrm{HP}-\beta-\mathrm{CD}(\delta=3.79 \mathrm{ppm}$ and $\delta$ $=3.83$ ppm, respectively). 


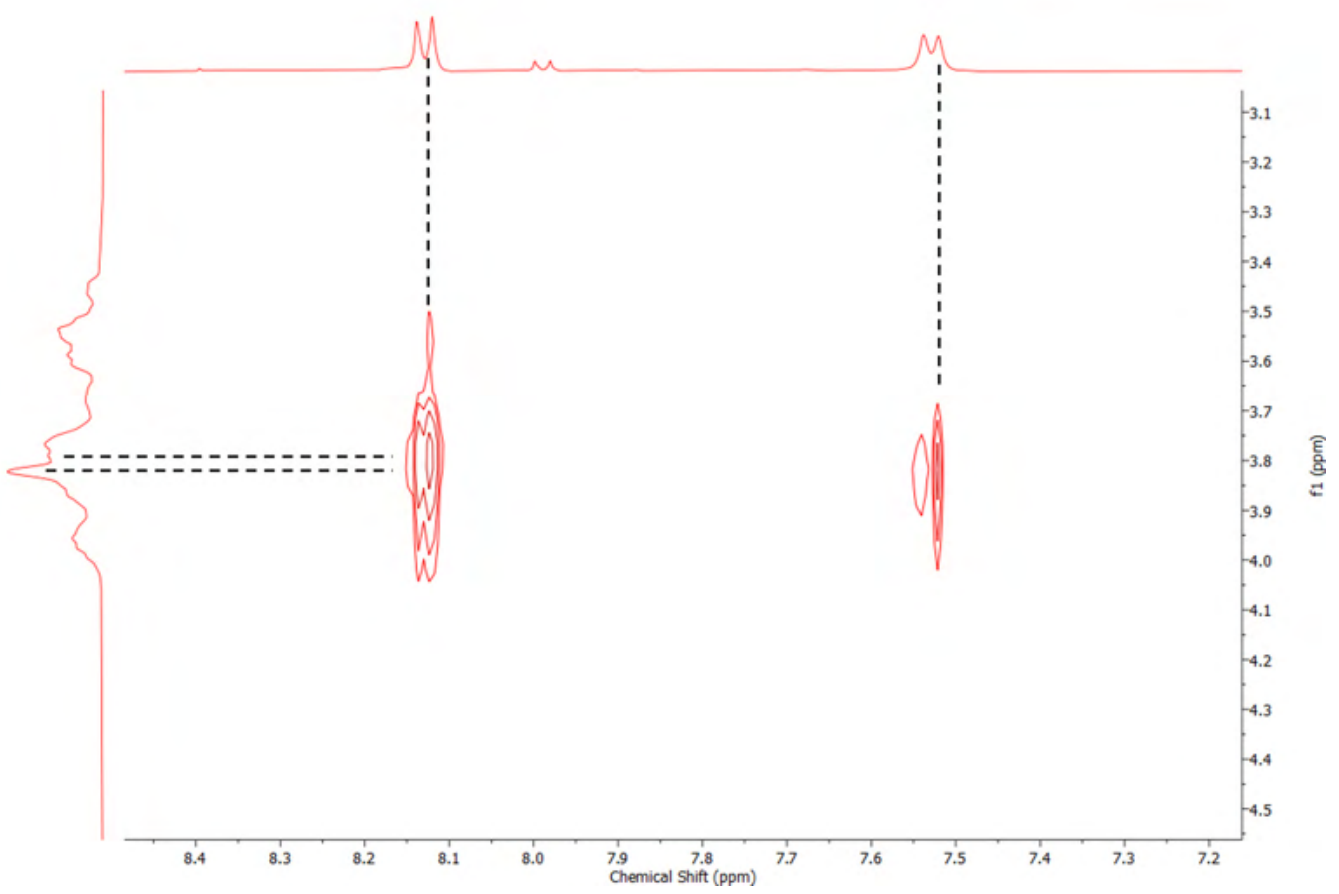

Figure S126. A magnified segment of the 2D NOESY ${ }^{1} \mathrm{H}-\mathrm{NMR}$ spectrum of the HP- $\beta-\mathrm{CD} \cdot 4$ complex, showing the cross-peak interactions between the signals of the aromatic protons of $4(\delta=7.52$ and $8.12 \mathrm{ppm})$ and the $\mathrm{H}^{5}$ and $\mathrm{H}^{6}$ protons of $\mathrm{HP}-\beta-\mathrm{CD}(\delta=3.79 \mathrm{ppm}$ and $\delta=3.83$ ppm, respectively). 


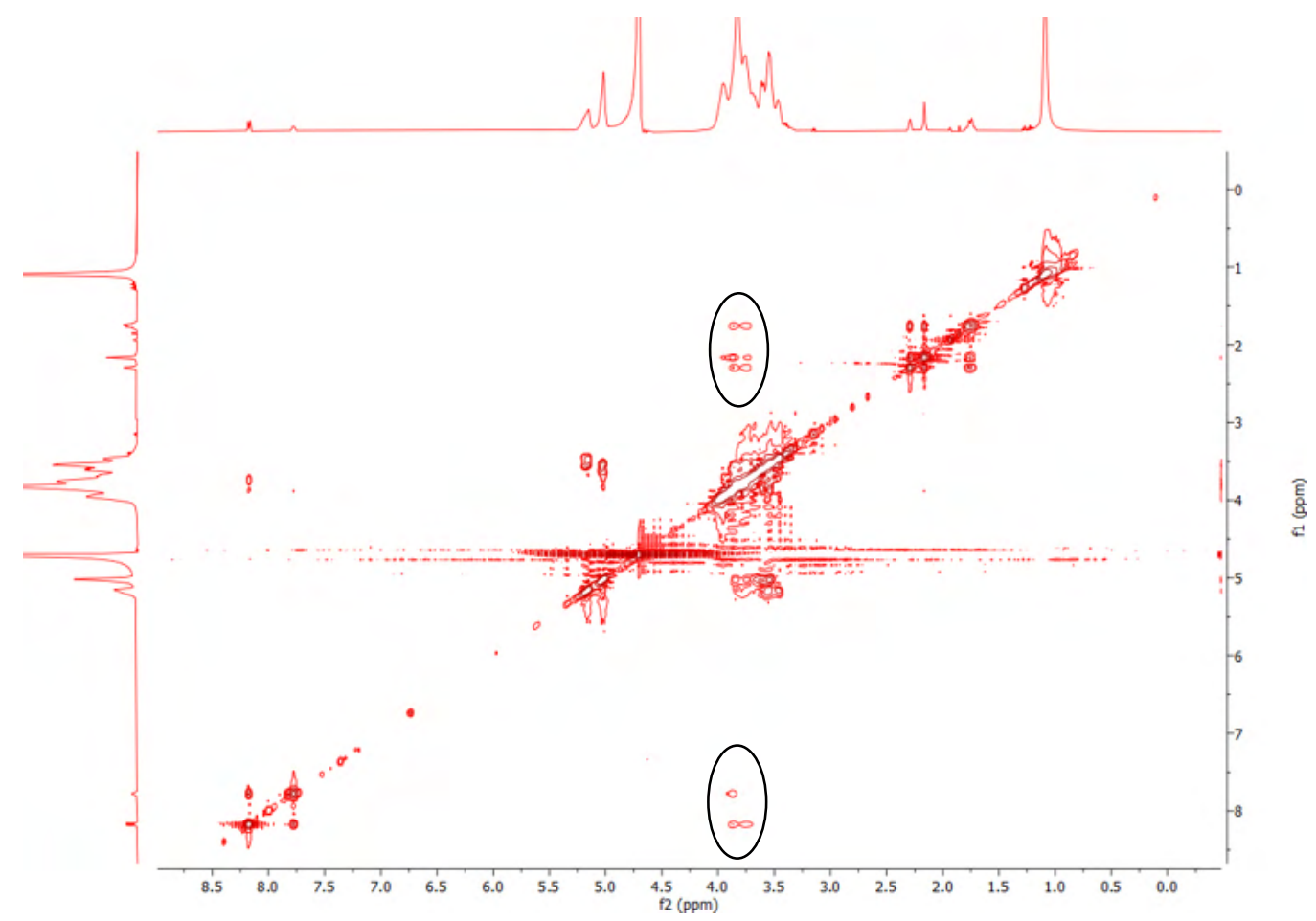

Figure S127. The 2D NOESY ${ }^{1} \mathrm{H}-\mathrm{NMR}$ spectrum of $\mathrm{HP}-\beta-\mathrm{CD} \cdot 6$ complex, in $\mathrm{D}_{2} \mathrm{O}$. Intermolecular cross-peak interactions between HP- $\beta-C D$ and 6 have been circled.

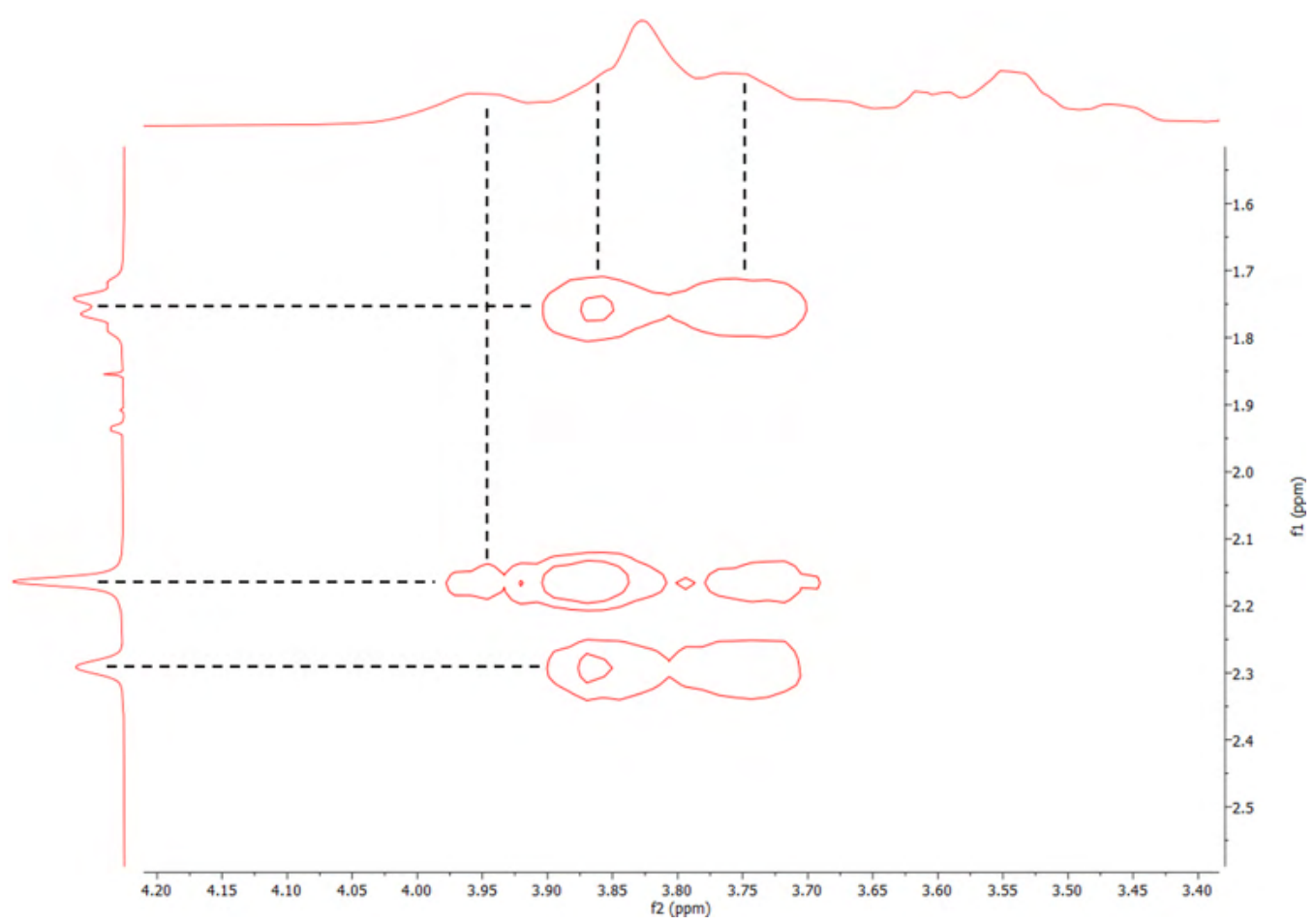

Figure S128. A magnified segment of the 2D NOESY ${ }^{1} \mathrm{H}-\mathrm{NMR}$ spectrum of the HP- $\beta-\mathrm{CD} \cdot 6$ complex, showing the cross-peak interactions between the signals of the adamantyl protons of $6(\delta=1.76,2.16$, and $2.29 \mathrm{ppm})$ and the $\mathrm{H}^{3}, \mathrm{H}^{5}$, and $\mathrm{H}^{8}$ protons of $\mathrm{HP}-\beta-\mathrm{CD}(\delta=3.85 \mathrm{ppm}, \delta$ $=3.75 \mathrm{ppm}$, and $\delta=3.95 \mathrm{ppm}$, respectively). 


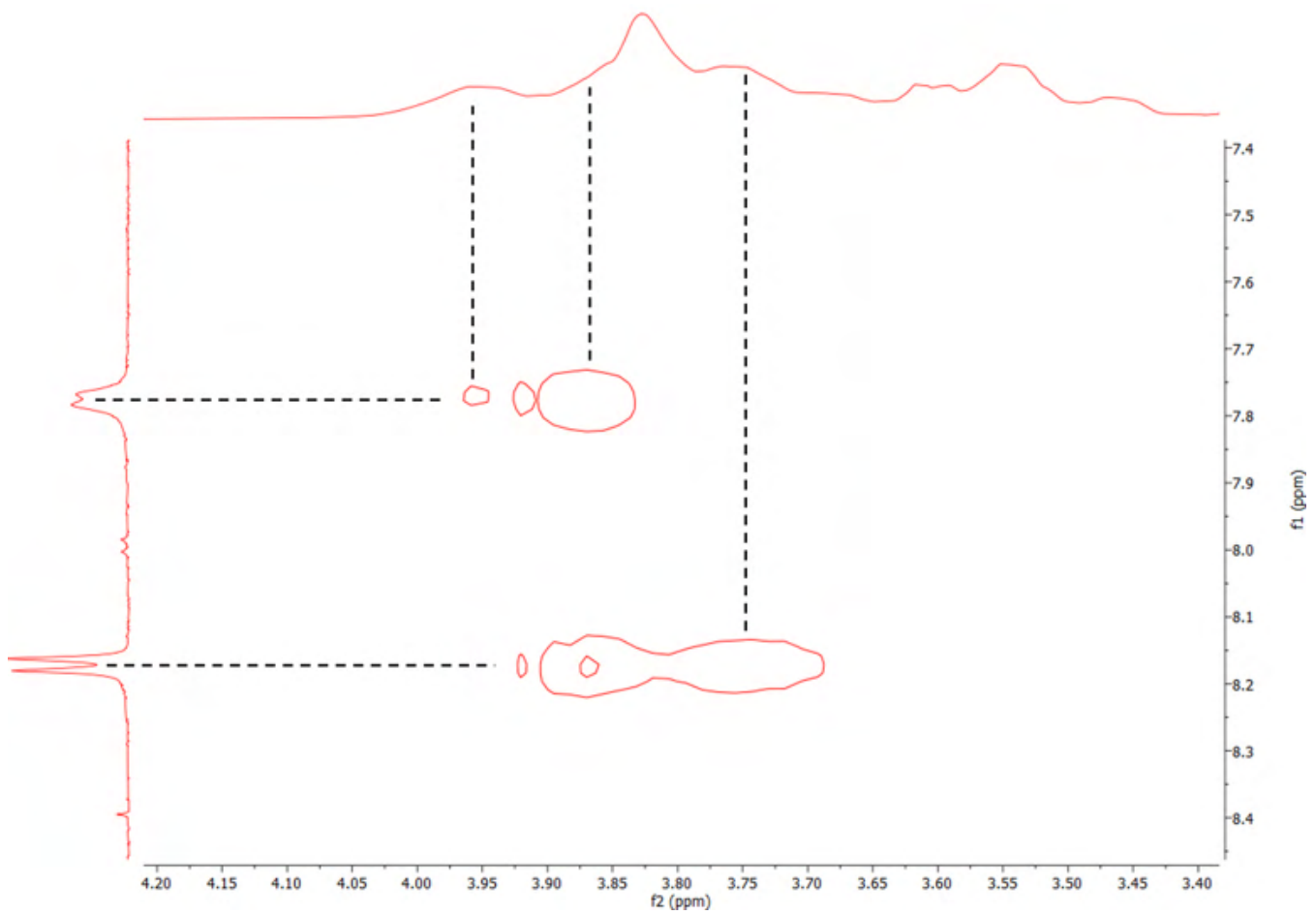

Figure S129. A magnified segment of the 2D NOESY ${ }^{1} \mathrm{H}-\mathrm{NMR}$ spectrum of the HP- $\beta-\mathrm{CD} \cdot 6$ complex, showing the cross-peak interactions between the signals of the aromatic protons of $6(\delta=7.78$ and $8.17 \mathrm{ppm})$ and the $\mathrm{H}^{3}, \mathrm{H}^{5}$, and $\mathrm{H}^{8}$ protons of $\mathrm{HP}-\beta-\mathrm{CD}(\delta=3.85 \mathrm{ppm}, \delta=3.75$ ppm, and $\delta=3.95$ ppm, respectively). 


\section{2. $\mathrm{Cl}^{-} / \mathrm{NO}_{3}{ }^{-}$Exchange Assay - Hill Analysis}

\subsection{General Vesicle Preparation}

Unilamellar vesicles were prepared following a procedure outlined previously by the Gale group. ${ }^{26}$ A chloroform solution of POPC $(37.5 \mathrm{mM}, 4 \mathrm{~mL})$ was transferred to a pre-weighed round-bottom flask, and the solvent was removed using a rotary evaporator. The pressure was lowered slowly to ensure the formation of a smooth lipid film. Subsequently, the film was dried in vacuo for 4-24 h, and the mass of lipid was recorded. The lipids were rehydrated with $4 \mathrm{~mL}$ of the respective internal solution (this number should correspond to the volume of POPC solution used initially) and vortexed until all lipids were removed from the sides of the flask and were suspended in solution. The lipids were subjected to 9 cycles of freeze-thaw by freezing using a dry ice/acetone bath and thawing in lukewarm water. Following this, the vesicles were left to rest at room temperature for $30 \mathrm{~min}$. The lipids were extruded through a $200 \mathrm{~nm}$ polycarbonate membrane 25 times to form monodisperse vesicles. Only $1 \mathrm{~mL}$ of solution was extruded at a time before being collected. Finally, any residual unencapsulated salt from the internal solution was either removed via dialysis for $12 \mathrm{~h}$ in the desired external solution or through size-exclusion using a B19 column packed with hydrated G-25 Sephadex®, which had been pre-saturated with the respective external solution. The lipid suspensions were diluted with the external solution to afford a stock solution $(10 \mathrm{~mL})$ of a known concentration.

\subsection{Electrode Calibration and Conversion of Raw Data}

An Accumet chloride ion-selective electrode (ISE) was used for both the $\mathrm{Cl}^{-} / \mathrm{NO}_{3}{ }^{-}$exchange assay and the cationophore coupled assay. Before the beginning of each set of experiments, calibration of the electrode was required to correct any drift between recorded datasets. The electrode was submerged in a sequence of $\mathrm{NaCl}$ solutions of increasing concentration (M), and the reading from the electrode was recorded after stabilising after 5-15 min. The electrode potential reading, $y$, was plotted against $\mathrm{NaCl}$ concentration $(\mathrm{M}), x$, and fitted to a simplified Nernst equation using Origin $2021 b$ (Academic). The equation is given as followed:

$$
y=\left(P_{1} \log _{10} x\right)+P_{2}
$$

Equation S7. Calibration of electrode potential data using a simplified Nernst equation. 
where $P_{1}$ and $P_{2}$ are the calibration parameters. The chloride ion concentration at any given time during the experiments can be calculated by substituting $P_{1}, P_{2}$ and $y$, the electrode potential reading, into the equation and solving for $x$, the chloride ion concentration. By subtracting the chloride concentration at $t=0$ from the concentration at a given time during the experiment, the total chloride concentration released from the vesicles at that given time can be calculated. Conversion to percentage chloride efflux was achieved by normalising the chloride concentration at time $(t)$ using the $100 \%$ chloride efflux value recorded at the end of the experiment ( $t=420 \mathrm{~s})$ when the vesicles were lysed with a water dispersion of Triton X$100(10 \% \mathrm{v} / \mathrm{v})$.

\subsection{Dose-Response Hill Analysis Assay Conditions}

Vesicles were prepared using an internal solution of $\mathrm{NaCl}(487 \mathrm{mM})$ and buffered to $\mathrm{pH} 7.2$ using a sodium phosphate salt buffer of $\mathrm{NaH}_{2} \mathrm{PO}_{4}(1.2 \mathrm{mM})$ and $\mathrm{Na}_{2} \mathrm{HPO}_{4}(4 \mathrm{mM})$. An external solution containing $\mathrm{NaNO}_{3}(487 \mathrm{mM})$ was prepared separately with the same sodium phosphate salt buffer and adjusted to $\mathrm{pH} 7.2$ using an aqueous solution of dilute $\mathrm{NaOH}$. Following the freeze-thaw cycles and extrusion, the $\mathrm{NaCl}$ lipids were dialysed in the $\mathrm{NaNO}_{3}$ external solution $(\sim 1600 \mathrm{~mL})$ for $12 \mathrm{~h}$. The lipid stock solution $(10 \mathrm{~mL})$ was diluted with the external solution to give both a concentration and volume suitable for ISE experiments $(1 \mathrm{mM}$, $5 \mathrm{~mL})$.

Each receptor was added as a DMSO solution $(10 \mu \mathrm{L})$ at $t=0 \mathrm{~s}$, and the change in chloride concentration was recorded using an Accumet chloride ISE. At $t=300 \mathrm{~s}$, a detergent solution (Triton X-100 (10\% v/v in $\left.\left.\mathrm{H}_{2} \mathrm{O}\right), 50 \mu \mathrm{L}\right)$ was injected to lyse the vesicles, and a final chloride concentration reading was recorded at $t=420 \mathrm{~s}$ to provide a $100 \%$ chloride efflux reading for use in calibration.

Dose-response experiments were performed at six transporter concentrations and a blank DMSO run, and percentage efflux at $t=270 \mathrm{~s}$ was recorded for each concentration tested. The chloride efflux (\%) at $t=270 \mathrm{~s}$ was plotted against receptor concentration (mol\%, with respect to lipid concentration). The recorded data was fit to the Hill Equation, using Origin 2021b (Academic), given as:

$$
y=V_{\max } \frac{x^{n}}{k^{n}+x^{n}}
$$


Equation S8. The Hill equation.

where $y$ represents the percentage of chloride efflux at $t=270 \mathrm{~s}, x$ is the transporter concentration (mol\%, with respect to lipids concentration), and $V_{\max }, k$ and $n$ are the parameters to be fitted. $V_{\max }$ represents the maximum chloride efflux (typically fixed to $100 \%$ ), $n$ can be interpreted as the Hill coefficient. A derived equation was used to calculate the $\mathrm{EC}_{50}$ value, the transporter concentration required to facilitate $50 \%$ chloride efflux, given as:

$$
\mathrm{EC}_{50}=k\left(\frac{50}{y_{1}-y_{0}-50}\right)^{\frac{1}{n}}
$$

Equation S9. The adapted EC50 equation.

where $k$ and $n$ are the derived parameters from the Hill equation, $y_{0}$ is the percentage chloride efflux at $t=0 \mathrm{~s}$, and $y_{1}$ is the percentage chloride efflux at $t=300 \mathrm{~s}$.

\subsection{Maximum Rate Calculations}

The maximum rate of chloride efflux was calculated for receptors 1, 2, 4-6, and inclusion complexes $\mathrm{HP}-\beta-\mathrm{CD} \cdot(\mathbf{1}, \mathbf{2}, \mathbf{4}, \mathbf{6})$ at $2 \mathrm{~mol} \%$, by fitting the efflux plot at the given concentration to Equation S10, in Origin 2021b (Academic), given as:

$$
y=A_{1} e^{\left(\frac{-x}{t_{1}}\right)}+A_{2} e^{\left(\frac{-x}{t_{2}}\right)}+y_{0}
$$

Equation S10. A non-linear exponential decay function.

where $y$ represents the percentage of chloride efflux $(\%), x$ is the receptor concentration $(\mathrm{mol} \%), y_{0}$ is the percentage chloride efflux at $t=0 \mathrm{~s}$, and $A_{1}, A_{2}, t_{1}$, and $t_{2}$ are the derived parameters. The maximum rate was obtained by calculating the first derivative at $x=0$, which is given as:

$$
K_{\text {initial }}=\frac{-A_{1}}{t_{1}}-\frac{A_{2}}{t_{2}}
$$

Equation S11. Derivative of the decay function used to determine the maximum rate given in $\% \mathrm{~s}^{-1}$.

The maximum rate of chloride efflux was calculated for receptor 3 at $2 \mathrm{~mol} \%$, by fitting the efflux plot at the given concentration to a non-linear Boltzmann curve function, in Origin 2021b (Academic), given as: 


$$
y=A_{2}+\frac{\left(A_{1}-A_{2}\right)}{\left(1+e^{\left(\frac{x-x_{0}}{d x}\right)}\right.}
$$

Equation S12. The Boltzmann function was used for the fitting of the efflux plots for compound 3.

where $y$ represents the percentage of chloride efflux (\%), $x$ is the receptor concentration ( $\operatorname{mol} \%)$, and $A_{1}, A_{2}, x_{0}$, and $d x$ are the derived parameters. The maximum rate was obtained by calculating the first derivative at $x=0$, which is given as:

$$
K_{\max }=\frac{A_{2}-A_{1}}{4 d x}
$$

Equation S13. Derivative of the Boltzmann function used to determine the maximum rate given in $\% \mathrm{~s}^{-1}$.

\section{5. $\mathrm{Cl}^{-} / \mathrm{NO}_{3}{ }^{-}$Exchange Assay - Efflux and Hill Plots}
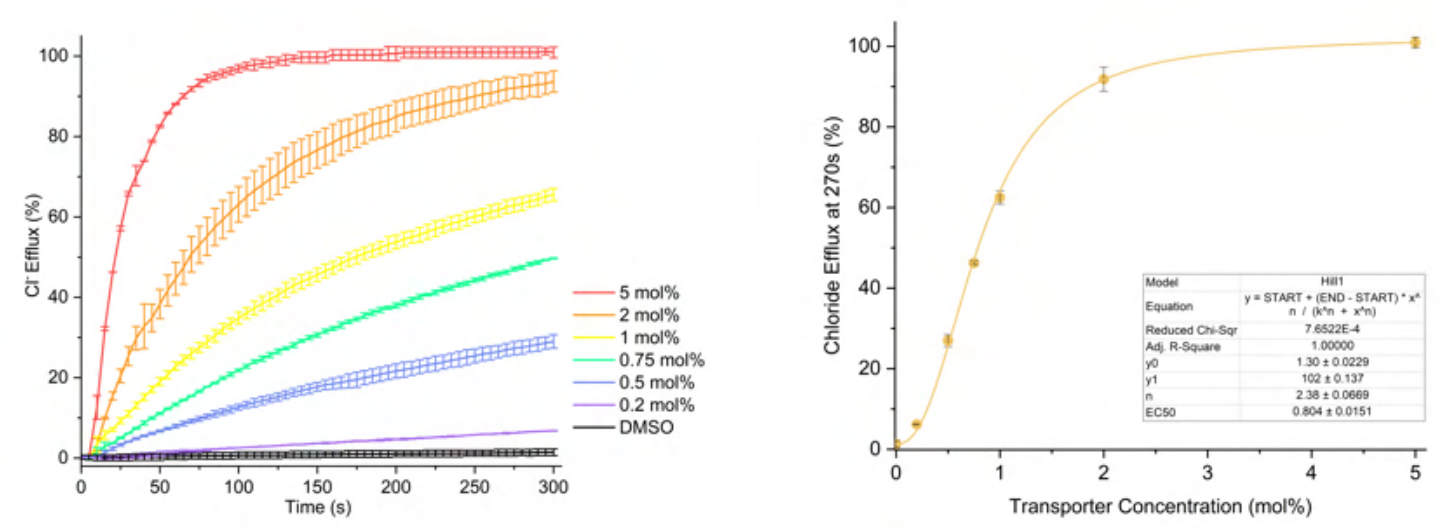

Figure S130. Hill analysis of $\mathrm{Cl}^{-} / \mathrm{NO}_{3}{ }^{-}$exchange facilitated by 1 . Each data point is the average of two repeats with error bars to show standard deviation. A run of pure DMSO was used as a control. 

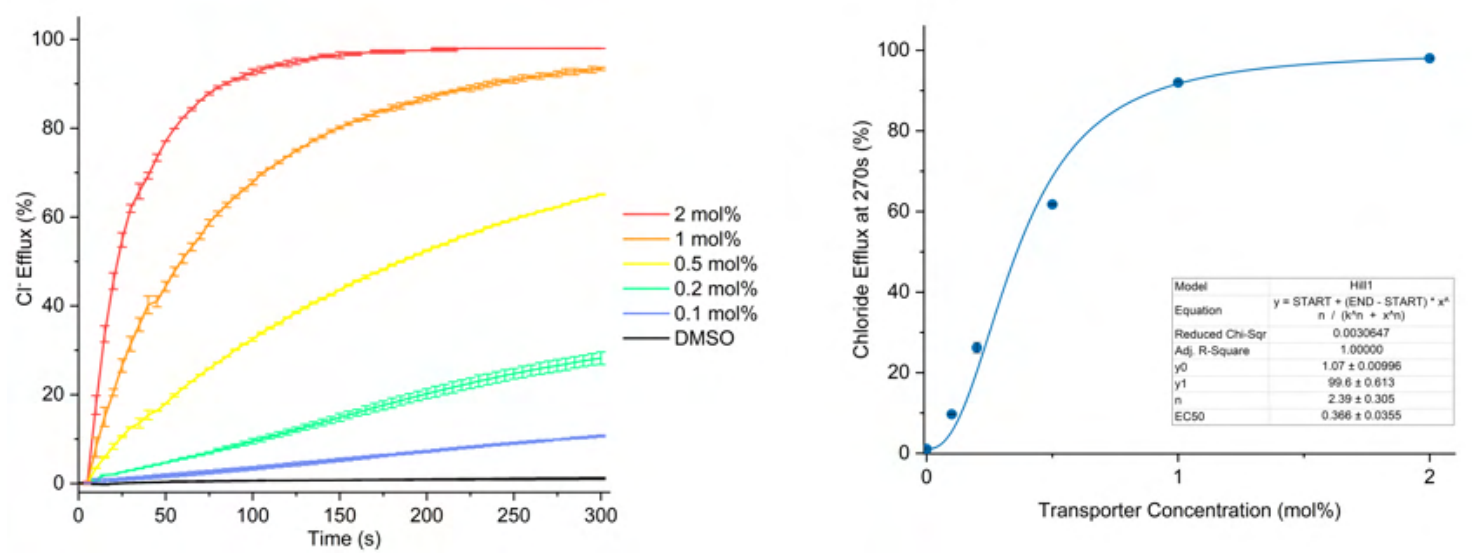

Figure S131. Hill analysis of $\mathrm{Cl} / \mathrm{NO}_{3}{ }^{-}$exchange facilitated by 2 . Each data point is the average of two repeats with error bars to show standard deviation. A run of pure DMSO was used as a control.
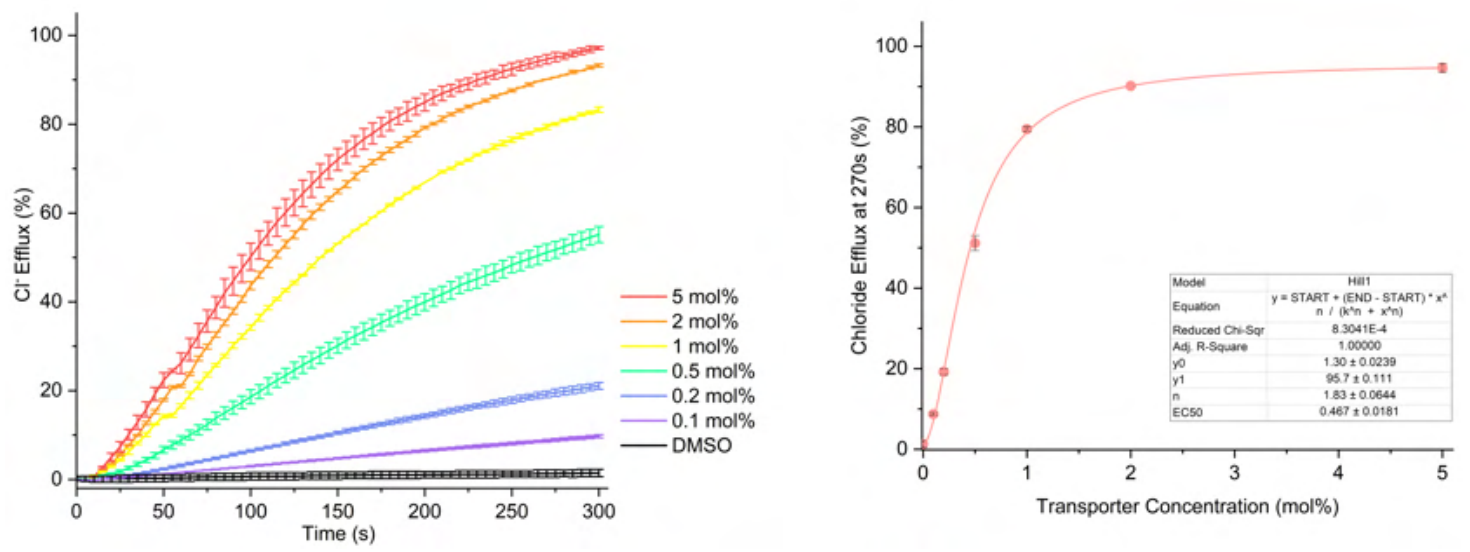

Figure S132. Hill analysis of $\mathrm{Cl} / \mathrm{NO}_{3}{ }^{-}$exchange facilitated by 3 . Each data point is the average of two repeats with error bars to show standard deviation. A run of pure DMSO was used as a control. 

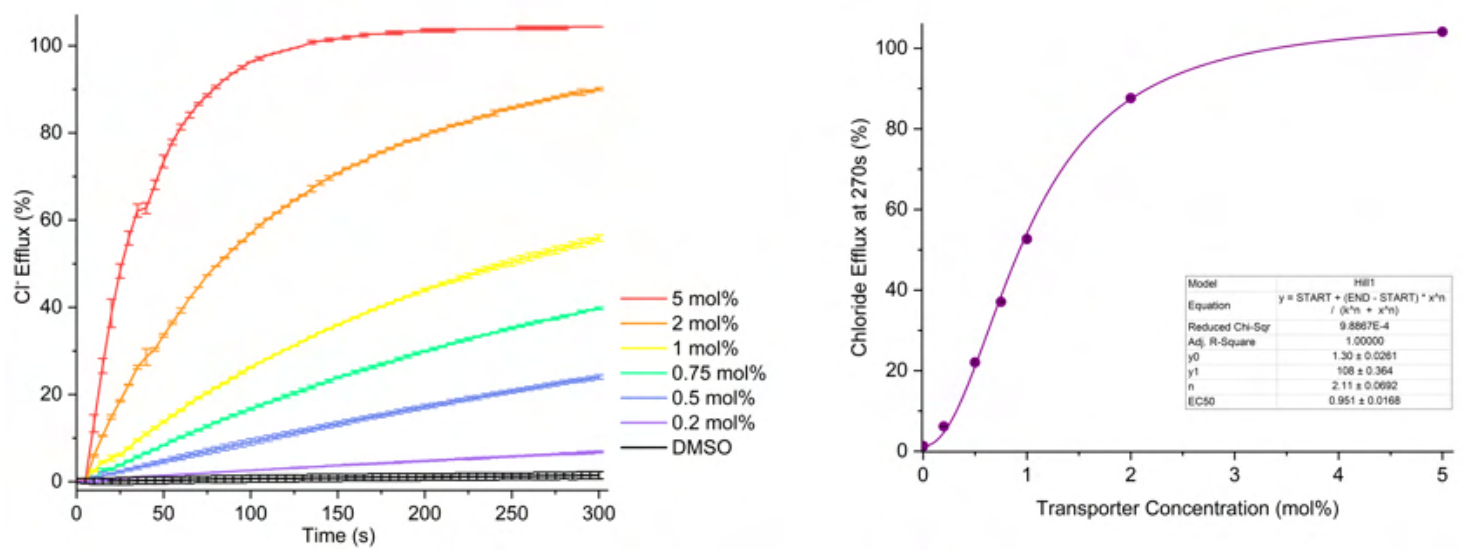

Figure S133. Hill analysis of $\mathrm{Cl} / \mathrm{NO}_{3}{ }^{-}$exchange facilitated by 4 . Each data point is the average of two repeats with error bars to show standard deviation. A run of pure DMSO was used as a control.
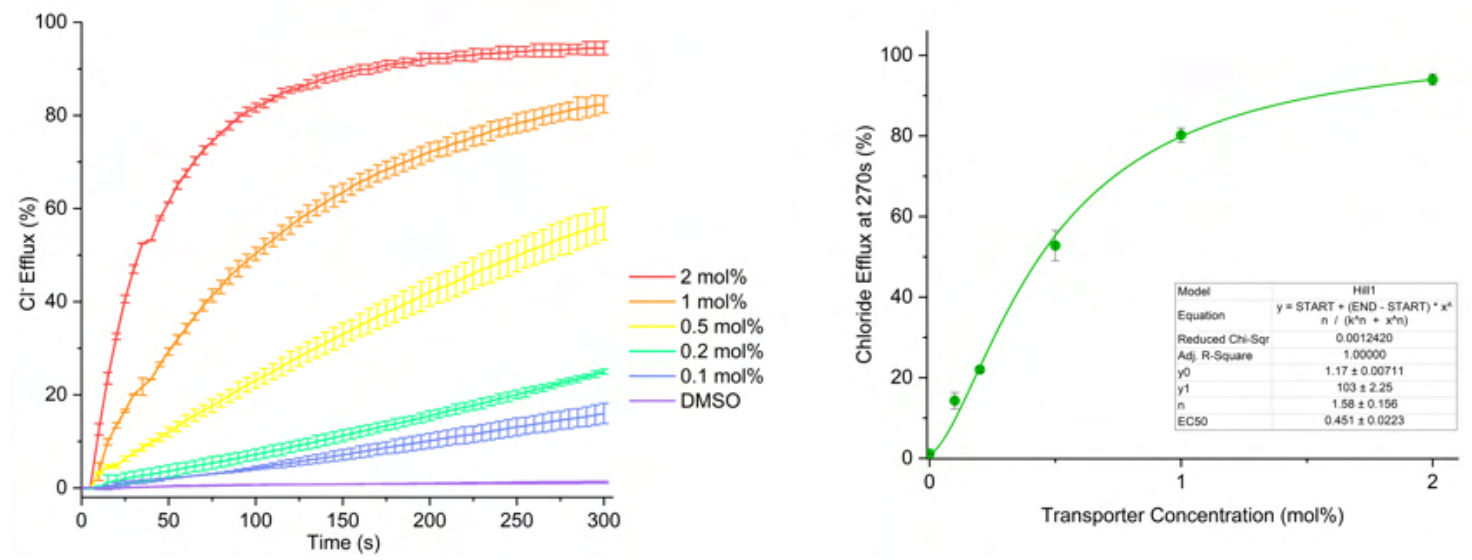

Figure S134. Hill analysis of $\mathrm{Cl} / \mathrm{NO}_{3}{ }^{-}$exchange facilitated by 5 . Each data point is the average of two repeats with error bars to show standard deviation. A run of pure DMSO was used as a control. 

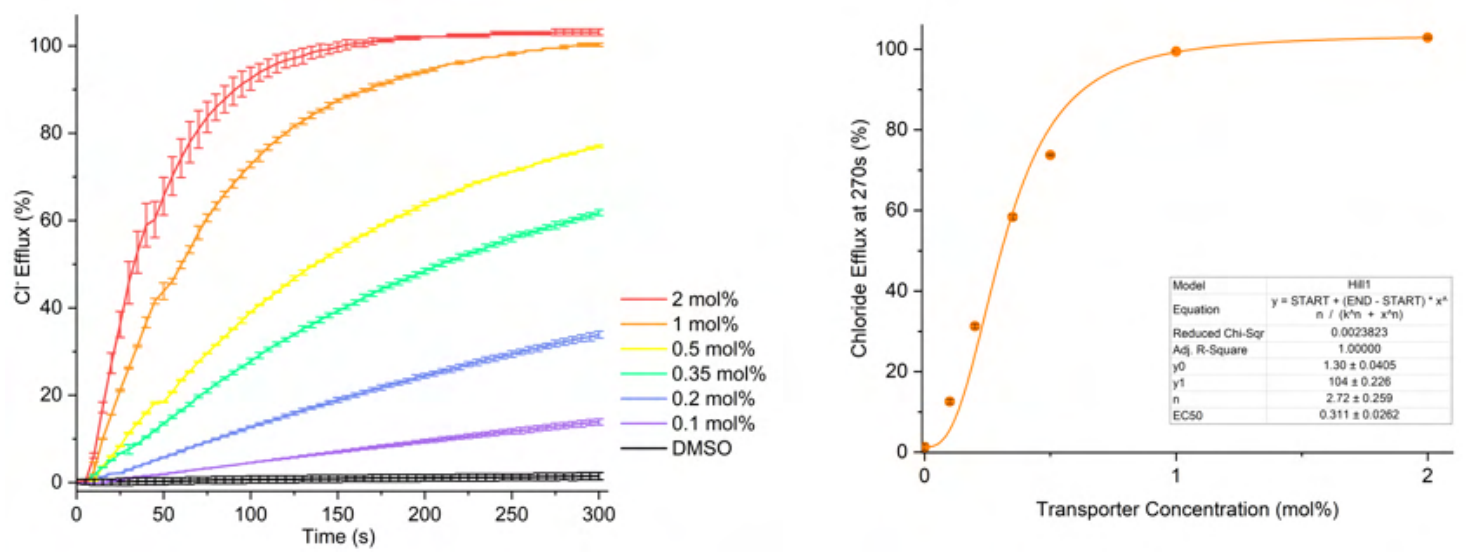

Figure S135. Hill analysis of $\mathrm{Cl}^{-} / \mathrm{NO}_{3}-$ exchange facilitated by 6 . Each data point is the average of two repeats with error bars to show standard deviation. A run of pure DMSO was used as a control.
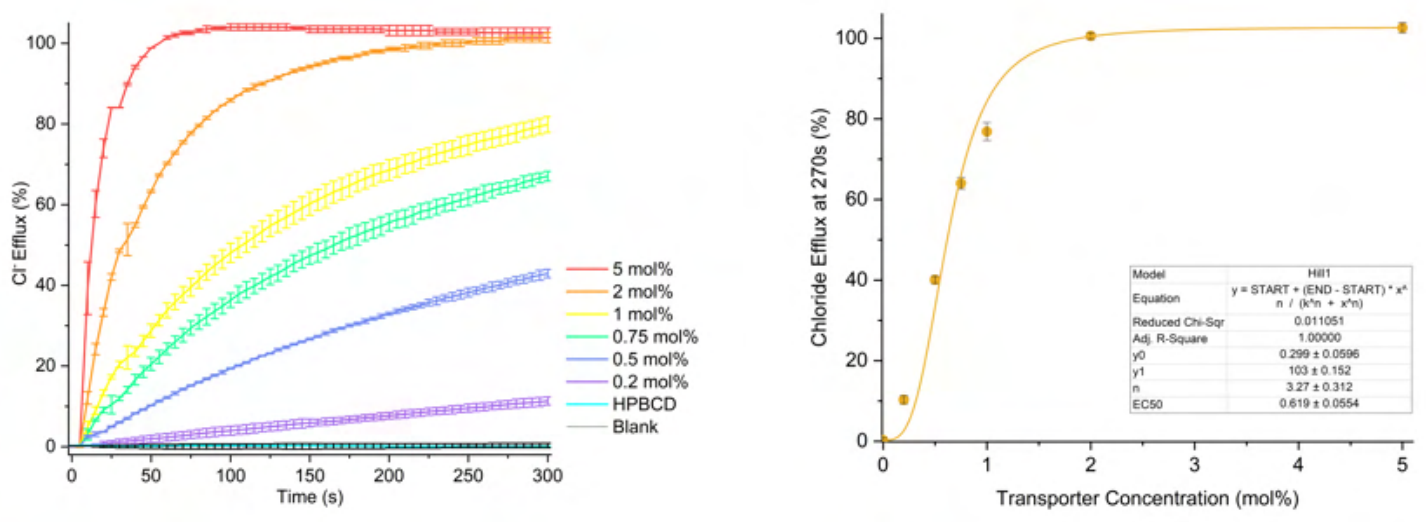

Figure S136. Hill analysis of $\mathrm{Cl} / \mathrm{NO}_{3}-$ exchange facilitated by the $\mathrm{HP}-\beta-\mathrm{CD} \cdot \mathbf{1}$ inclusion complex. Each data point is the average of two repeats with error bars to show standard deviation. A run of pure deionized $\mathrm{H}_{2} \mathrm{O}$ and a run of solely $\mathrm{HP}-\beta-\mathrm{CD}$ were used as controls. 

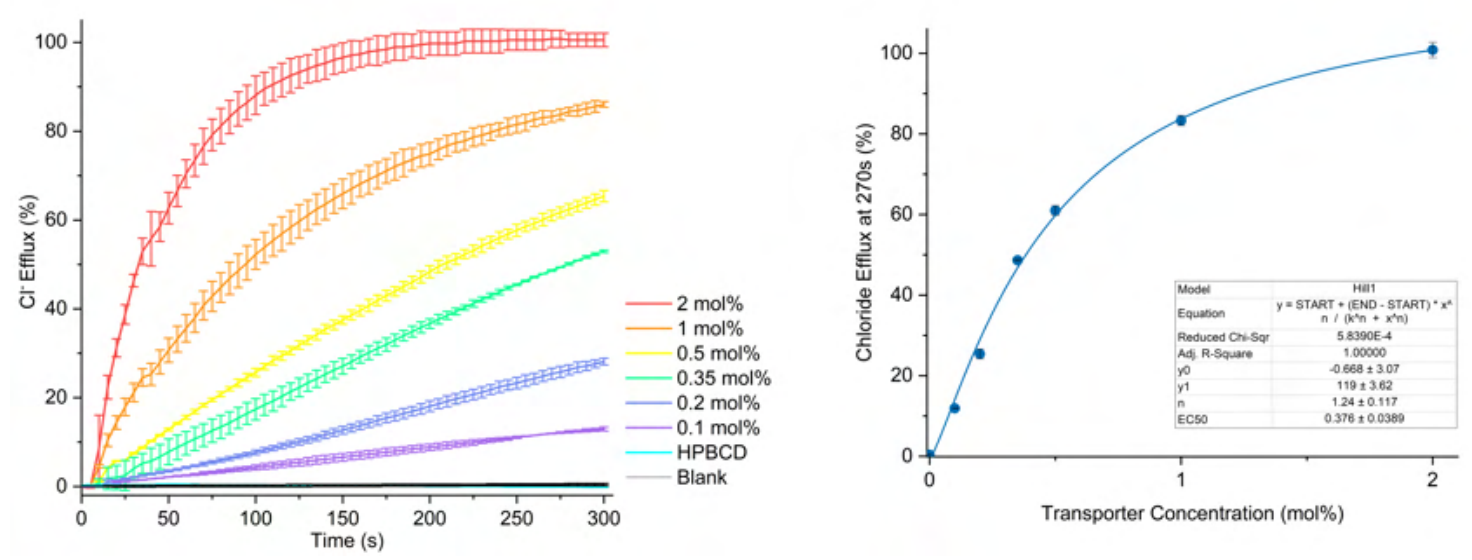

Figure S137. Hill analysis of $\mathrm{Cl}^{-} / \mathrm{NO}_{3}^{-}$exchange facilitated by the $\mathrm{HP}-\beta-\mathrm{CD} \cdot 2$ inclusion complex. Each data point is the average of two repeats with error bars to show standard deviation. A run of pure deionized $\mathrm{H}_{2} \mathrm{O}$ and a run of solely $\mathrm{HP}-\beta-\mathrm{CD}$ were used as controls.
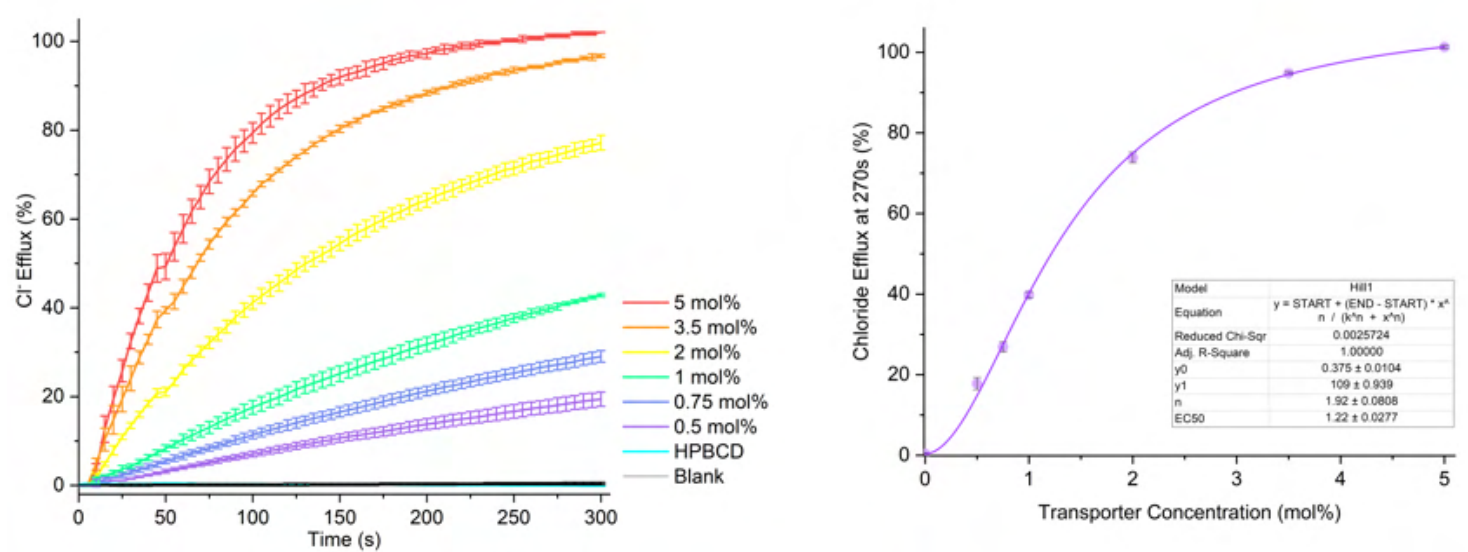

Figure S138. Hill analysis of $\mathrm{Cl}-\mathrm{NO}_{3}-$ exchange facilitated by the $\mathrm{HP}-\beta-\mathrm{CD} \cdot 4$ inclusion complex. Each data point is the average of two repeats with error bars to show standard deviation. A run of pure deionized $\mathrm{H}_{2} \mathrm{O}$ and a run of solely $\mathrm{HP}-\beta-\mathrm{CD}$ were used as controls. 

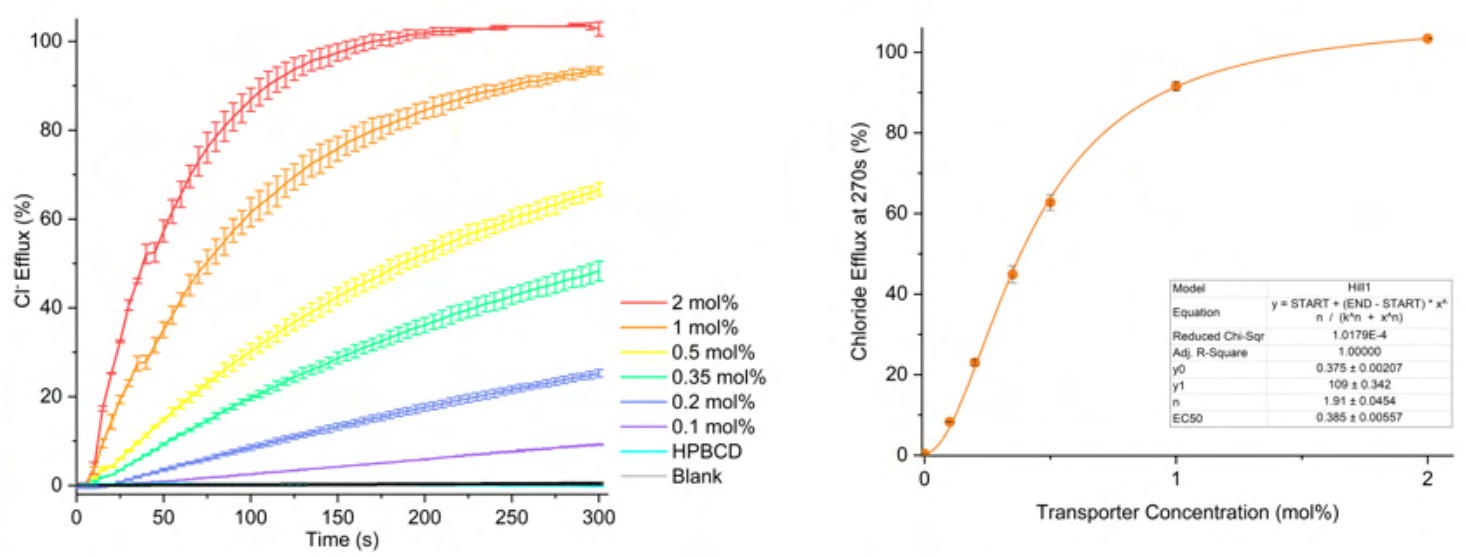

Figure S139. Hill analysis of $\mathrm{Cl}^{-} / \mathrm{NO}_{3}^{-}$exchange facilitated by the $\mathrm{HP}-\beta-\mathrm{CD} \cdot 6$ inclusion complex. Each data point is the average of two repeats with error bars to show standard deviation. A run of pure deionized $\mathrm{H}_{2} \mathrm{O}$ and a run of solely $\mathrm{HP}-\beta-\mathrm{CD}$ were used as controls.

\section{References}

1. D. Brynn Hibbert and P. Thordarson, Chem. Commun., 2016, 52, 12792-12805.

2. J. Szejtli, Chem. Rev., 1998, 98, 1743-1754.

3. J. C. Harrison and M. R. Eftink, Biopolymers, 1982, 21, 1153-1166.

4. G. Tiwari, R. Tiwari and A. K. Rai, J. Pharm. Bioallied Sci., 2010, 2, 72-79.

5. S. Gould and R. C. Scott, Food Chem. Toxicol., 2005, 43, 1451-1459.

6. M. E. Bellringer, T. G. Smith, R. Read, C. Gopinath and P. Olivier, Food Chem. Toxicol., 1995, 33, 367-376.

7. M. E. Brewster, K. S. Estes and N. Bodor, Int. J. Pharm., 1990, 59, 231-243.

8. F. J. O. Espinar, S. A. Igea, J. B. Méndez and J. L. V. Jato, Int. J. Pharm., 1991, 70, 35-41.

9. C.-W. Lee, S.-J. Kim, Y.-S. Youn, E. Widjojokusumo, Y.-H. Lee, J. Kim, Y.-W. Lee and R. R. Tjandrawinata, J. Supercrit. Fluids, 2010, 55, 348-357.

10. M. Stojanov, R. Wimmer and K. L. Larsen, J. Pharm. Sci., 2011, 100, 3177-3185.

11. T. C. Chen, S.-C. Yu, C.-M. Hsu, F.-J. Tsai and Y. Tsai, J. Incl. Phenom. Macrocycl. Chem., 2017, 88, 27-34.

12. K. P. Sambasevam, S. Mohamad, N. M. Sarih and N. A. Ismail, Int. J. Mol. Sci., 2013, 14.

13. S. Riela, G. Lazzara, P. Lo Meo, S. Guernelli, F. D'Anna, S. Milioto and R. Noto, Supramol. Chem., 2011, 23, 819-828.

14. J. Pitha, J. Milecki, H. Fales, L. Pannell and K. Uekama, Int. J. Pharm., 1986, 29, 73-82.

15. W. M. Haynes, CRC handbook of chemistry and physics, CRC press, 2014. 
16. A. Rostami, A. Colin, X. Y. Li, M. G. Chudzinski, A. J. Lough and M. S. Taylor, JOC, 2010, 75, 3983-3992.

17. Á. Nacsa, R. Ambrus, O. Berkesi, P. Szabó-Révész and Z. Aigner, J. Pharm. Biomed. Anal., 2008, 48, 1020-1023.

18. Y.-1. Shen, S.-h. Yang, L.-m. Wu and X.-y. Ma, Spectrochim. Acta. A Mol. Biomol. Spectrosc., 2005, 61, 1025-1028.

19. A. Figueiras, L. Ribeiro, M. T. Vieira and F. Veiga, J. Incl. Phenom. Macrocycl. Chem., 2007, 57, 173-177.

20. S. Prabu, M. Swaminathan, K. Sivakumar and R. Rajamohan, J. Mol. Struct., 2015, 1099, 616-624.

21. M. Shanmugam, D. Ramesh, V. Nagalakshmi, R. Kavitha, R. Rajamohan and T. Stalin, Spectrochim. Acta. A Mol. Biomol. Spectrosc., 2008, 71, 125-132.

22. P. Mura, J. Pharm. Biomed. Anal., 2015, 113, 226-238.

23. O. Adeoye, C. Costa, T. Casimiro, A. Aguiar-Ricardo and H. Cabral-Marques, J. Supercrit. Fluids, 2018, 133, 479-485.

24. P. P. Menezes, M. R. Serafini, B. V. Santana, R. S. Nunes, L. J. Quintans, G. F. Silva, I. A. Medeiros, M. Marchioro, B. P. Fraga, M. R. V. Santos and A. A. S. Araújo, Thermochim. Acta, 2012, 548, 45-50.

25. D. Neuhaus, eMagRes, 2007.

26. L. A. Jowett and P. A. Gale, Supramol. Chem., 2019, 31, 297-312. 